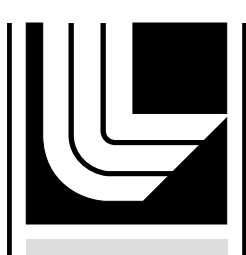

LAWRENCE LIVERMORE NATIONAL LABORATORY

Mapping Diffuse Seismicity for Geothermal Reservoir Management with Matched Field Processing: Year 2 Report

D. C. Templeton, D. B. Harris, J. Wang

April 15, 2013 
This document was prepared as an account of work sponsored by an agency of the United States government. Neither the United States government nor Lawrence Livermore National Security, LLC, nor any of their employees makes any warranty, expressed or implied, or assumes any legal liability or responsibility for the accuracy, completeness, or usefulness of any information, apparatus, product, or process disclosed, or represents that its use would not infringe privately owned rights. Reference herein to any specific commercial product, process, or service by trade name, trademark, manufacturer, or otherwise does not necessarily constitute or imply its endorsement, recommendation, or favoring by the United States government or Lawrence Livermore National Security, LLC. The views and opinions of authors expressed herein do not necessarily state or reflect those of the United States government or Lawrence Livermore National Security, LLC, and shall not be used for advertising or product endorsement purposes.

This work performed under the auspices of the U.S. Department of Energy by Lawrence Livermore National Laboratory under Contract DE-AC52-07NA27344. 


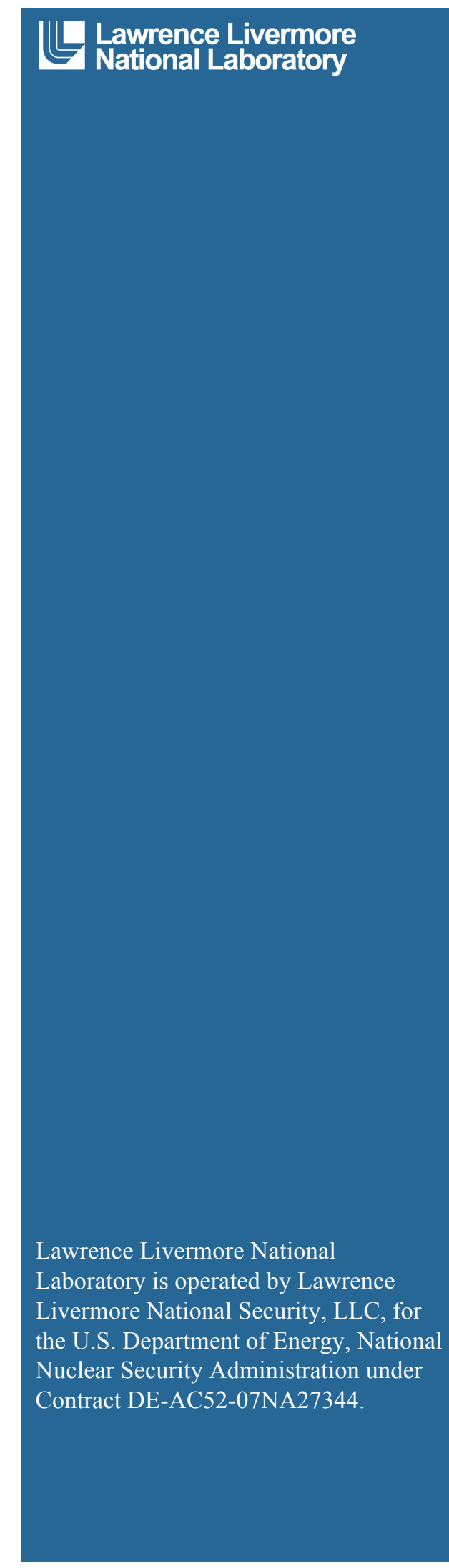

\section{Mapping Diffuse Seismicity for Geothermal Reservoir Management with Matched Field Processing: Year 2 Report}

Unclassified

September 30, 2011

Dennise C. Templeton, David B. Harris, Jingbo Wang

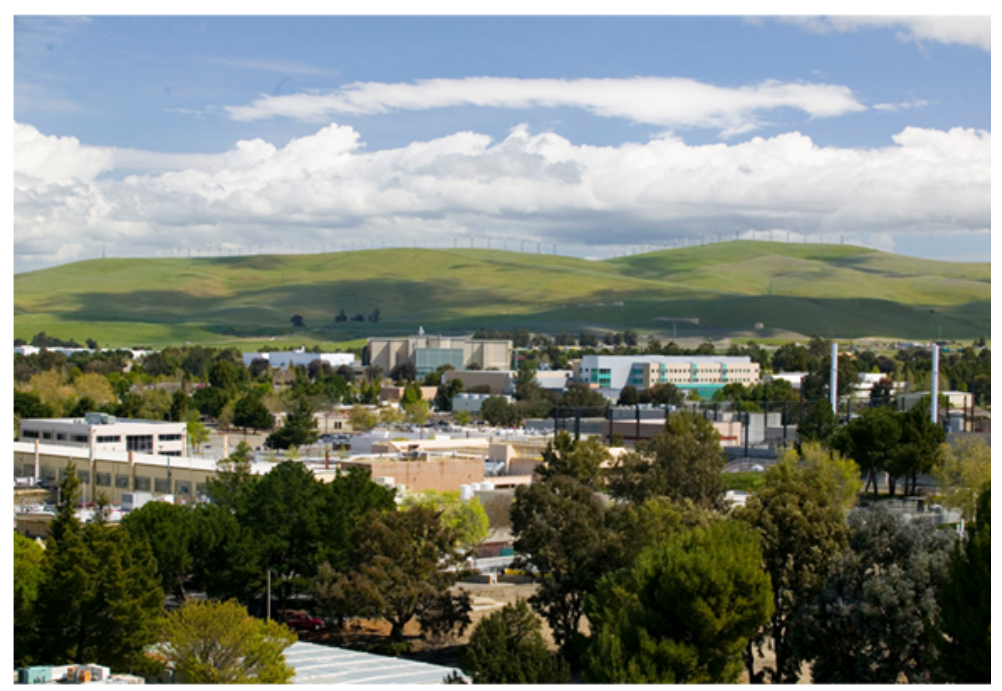




\section{Disclaimer}

This document was prepared as an account of work sponsored by an agency of the United States government. Neither the United States government nor Lawrence Livermore National Security, LLC, nor any of their employees makes any warranty, expressed or implied, or assumes any legal liability or responsibility for the accuracy, completeness, or usefulness of any information, apparatus, product, or process disclosed, or represents that its use would not infringe privately owned rights. Reference herein to any specific commercial product, process, or service by trade name, trademark, manufacturer, or otherwise does not necessarily constitute or imply its endorsement, recommendation, or favoring by the United States government or Lawrence Livermore National Security, LLC. The views and opinions of authors expressed herein do not necessarily state or reflect those of the United States government or Lawrence Livermore National Security, LLC, and shall not be used for advertising or product endorsement purposes. 


\section{Table of Contents}

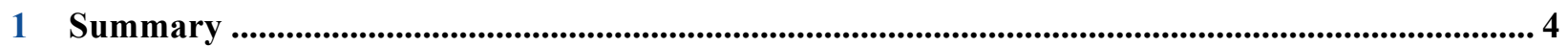

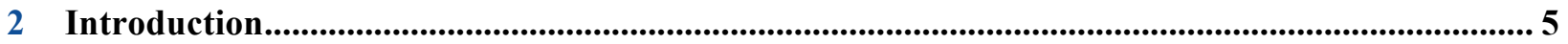

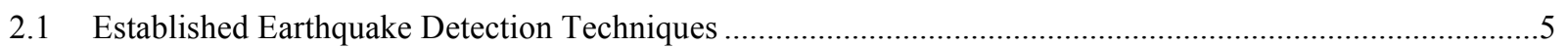

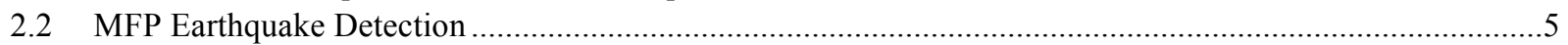

3 Test Case: Salton Sea Geothermal Field ......................................................................................... 6

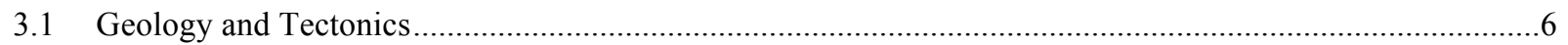

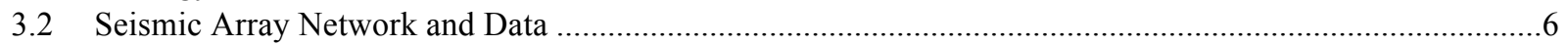

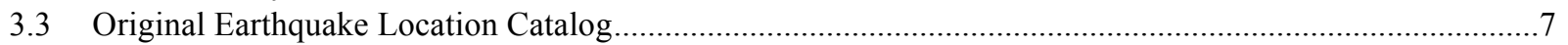

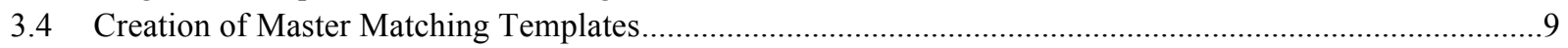

3.5 Application of MFP Methodology to the Continuous Seismic Data ….....................................................

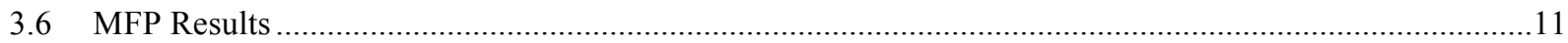

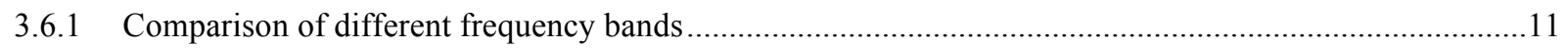

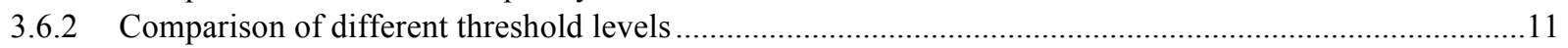

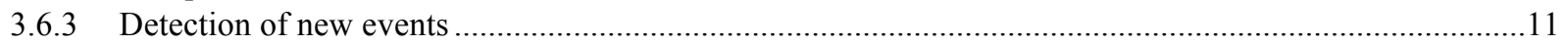

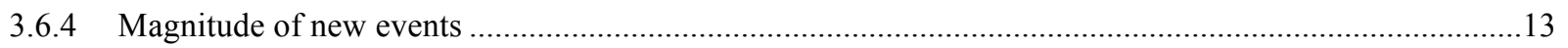

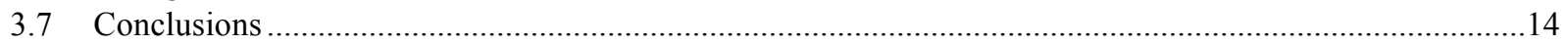

4 Salton Sea Geothermal Field Coulomb Stress Investigation .......................................................... 15

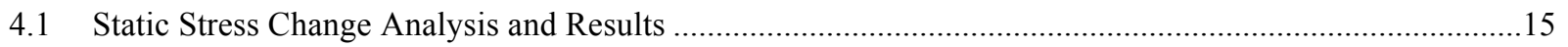

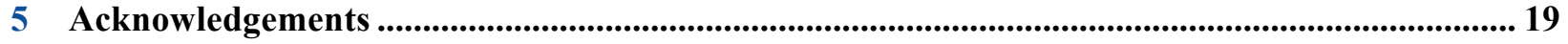

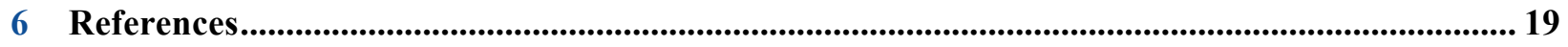

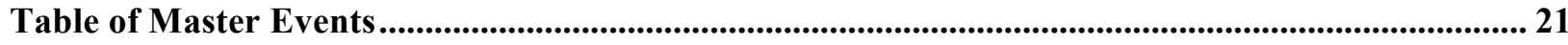

List of New Events Identified In This Study............................................................................................ 27 


\section{$1 \quad$ Summary}

Detecting and locating seismic events in managed underground geothermal reservoirs can become increasingly difficult during certain reservoir management operations. For example, during fluid injection procedures, large numbers of microearthquakes frequently occur during a relatively short amount of time. On seismic records, these events can overlap or have poor signal-to-noise ratios (SNR). This can obscure the onset of smaller signals and complicate the picking of seismic phases needed for earthquake location algorithms. To accurately illuminate the active areas of the underground reservoir through time however, a complete earthquake catalog down to very small magnitudes is necessary.

To aid in the seismic characterization of reservoir fracture networks, we propose to complement traditional earthquake detection and location techniques with the empirical matched field processing (MFP) method. The principle of the empirical MFP method is to calculate propagation from source to receivers for pre-existing events, also called master templates. Then MFP matches the spatial structure of incoming seismicity observed by a network of sensors to master templates keyed to potential event locations.

We demonstrate that empirical MFP can complement existing catalogs and techniques by using archived seismic information to increase earthquake catalog completeness. We identify and construct representative master templates using the Southern California Earthquake Data Center (SCEDC) earthquake catalog and archived waveform data of events originating within the Salton Sea Geothermal Field (SSGF). We identify 231 master events that have good quality seismic records at four or more seismic stations. We apply the empirical MFP method to continuous seismic data collected at the SSGF between November 2009 and December 2010. The MFP method successfully identified 5357 local events, while the original catalog only reported 1536 events. This increase in the number of events allows us to better determine the active fractures of the geothermal reservoir.

We model the Coulomb stress change due to events between M2 - M3.5 that occurred during the January 2010 seismic swarm. Our results show a good correlation between regions of increased Coulomb stress change due to double-couple shear events on faults and the locations of subsequent microseismicity. In addition, we investigate the lower event detection magnitude threshold of our surface network using the MFP method. It clearly shows the event detection threshold is extended to include many smaller earthquakes with magnitudes down to M0.0. Therefore, we believe that the empirical MFP method, when combined with conventional methods, significantly improves network detection capabilities. 


\section{Introduction}

\subsection{Established Earthquake Detection Techniques}

Conventional earthquake detection algorithms typically employ short-term average - long-term average (STA/LTA) triggers to detect microearthquakes on seismic recorders (Ambuter and Solomon, 1974). These techniques require accurate picking of individual seismic phase onsets across a network of sensors and work best on seismic records containing a single well-recorded event with high signal-to-noise ratio. Seismic phase picking, however, can become difficult or impossible on seismic records containing large numbers of overlapping events or events with poor signal-to-noise ratios. In such cases as these, may events may either be missed entirely or if they are detected, they may be unable to be located with any certainty.

Advanced earthquake detection techniques include the use of correlation detectors to identify events (such as Gibbons and Ringdal, 2006; Gibbons et al., 2007). These define spatial-temporal waveform templates and screen the continuous data stream to detect events that correlate with these templates. The matched filter technique using the waveform cross-correlation mechanism is also widely applied to detect weak tremor signals (Shelly et. al. 2006) and aftershocks (Peng and Zhao, 2009; Harris and Dodge, 2011). Cross-correlation techniques such as these obviate loss of coherence across an aperture by calibrating simultaneously the temporal and spatial structure of signals from specific repeating sources. However, the applicability of correlation methods is limited by variations in source characteristics (time history, mechanism and physical dimension), which change the observed waveform (Harris and Kvaerna, 2010).

\subsection{MFP Earthquake Detection}

Our MFP technique differs from the established earthquake detection techniques and is an adaptation of a signal processing technique originally developed to locate continuous underwater acoustic sources [Bucker 1976; Baggeroer et al. 1993]. We calculate the wavefield structure across an array by estimating the structure directly from field calibration data, i.e., previously observed seismic events. Then we steer the array explicitly in the frequency domain using the complex phase and amplitude factors obtained from the field data (Harris and Kvaerna, 2010). We refer to this strategy as empirical MFP, in which the master templates created from the seismograms of previously detected micro-earthquakes contain contributions from direct and scattered seismic energy.

Empirical MFP largely eliminates the sensitivity of (correlation) matching operations to source time history variations by processing the observed data stream in a large number of narrow frequency bands. This makes MFP sensitive to the spatial structure of the signal at the observing aperture (controlled by mechanism and propagation), but not the temporal structure (controlled, in part, by source time history). In this way MFP can identify previously undiscovered events even if they bear little resemblance to the master event in the time domain. 
The sensor data are decomposed into a collection of complex-analytic, narrowband components with an efficient filterbank algorithm [Portnoff, 1980]. Beamforming is performed within each narrow band by weighting the complex-analytic sensor traces with complex scale factors and summing the weighted traces over the receiving aperture. We determine the wideband detection statistic by computing the squared magnitude of each narrowband beam to form envelopes proportional to beam power, then summing the resulting power traces over all bands.

For any particular band, the vector of complex weights is obtained as the principal eigenvector of a narrowband covariance matrix estimated from a master event. The principal eigenvector essentially calibrates the collection of Fourier transforms (evaluated at the band center frequency) of Greens functions governing propagation from the location of the master event(s) to the observing sensors. For the complex weighting operation on the complex-analytic, narrowband traces to approximate a time delay, the width of the narrow bands must be small: the reciprocal of the bandwidth (a time quantity) must be large compared to the largest propagation delay across the observing aperture. A consequence of this requirement is that bandwidths on the order of $0.1 \mathrm{~Hz}$ or less are required for processing data from local networks with an aperture on the order of several kilometers. This requirement limits the temporal resolution of the method and thus origin time estimates are poorly resolved.

\section{Test Case: Salton Sea Geothermal Field}

\subsection{Geology and Tectonics}

The Salton Sea geothermal field (SSGF) is a liquid-dominated geothermal system located in Southern California at the southern end of the Salton Sea. A portion of the SSGF is situated within the Brawley Seismic Zone, which extends across the Salton Sea to connect with the San Andreas Fault to the north and the Imperial Fault to the south [Fuis and Mooney, 1990]. This zone is the most northerly of a series of spreading centers extending down into the Gulf of California that can be associated with the East Pacific Rise [Fuis et al., 1984]. These extensional tectonic areas allow magmatic intrusions into the crust, providing heat sources that drive the observed geothermal activity.

\subsection{Seismic Array Network and Data}

A local surface seismic monitoring network (the EN network) monitors SSGF seismic activity. The EN network is composed of eight three-component seismic stations (Figure 1). The stations are instrumented with $4.5 \mathrm{~Hz}$ L15B three-component geophones with Quanterra Q330 dataloggers. The sample rate of the continuous data is 100 samples per second (SPS). Since January 2008, continuous data from this network has been archived and freely available at the Southern California Earthquake Data Center (SCEDC). 


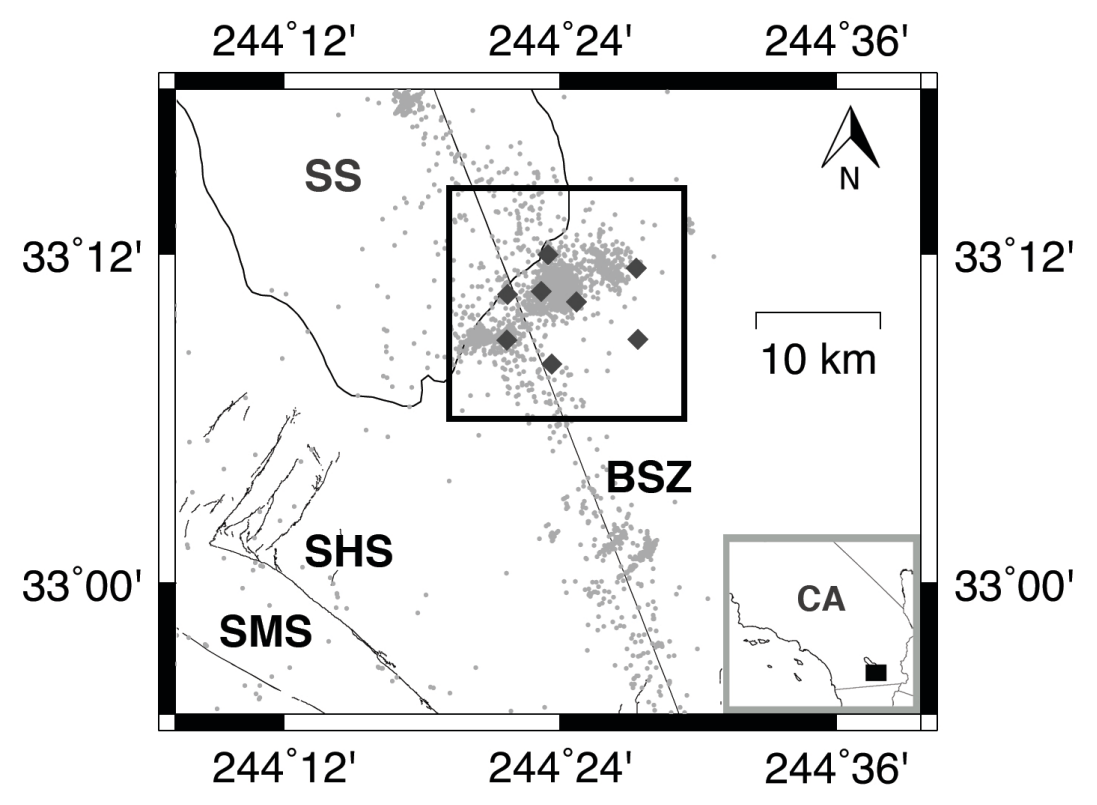

Figure 1. Map showing study area indicated by a grey box, EN network seismic stations indicated by dark grey diamonds, major faults indicated by grey lines and background seismicity indicated by grey dots. Inset map shows the location of the study area in Southern California. SS = Salton Sea, BSZ = Brawley Seismic Zone SHS = Superstition Hill segment, $S M S=$ Superstition Mountains segment, $C A=$ California.

For the event locations, we have both the original solutions from the SCEDC catalog and the waveform cross-correlation relocation results of Hauksson et al. [2012]. As the catalog of Hauksson et al. [2012] provides a better relative location of the events, we plot all events using the locations from the Hauksson et al. [2012] catalog. The continuous seismic data is obtained from all available stations in the EN network from the SCEDC database.

\subsection{Original Earthquake Location Catalog}

The time period of this test case spanned between November 2009 and December 2010. The original SCEDC earthquake catalog identified 1536 events occurring in the SSGF during this time frame (Figure 2). The data during this time period includes microearthquake information from two large seismic swarms occurring in November 2009 and January 2010. 


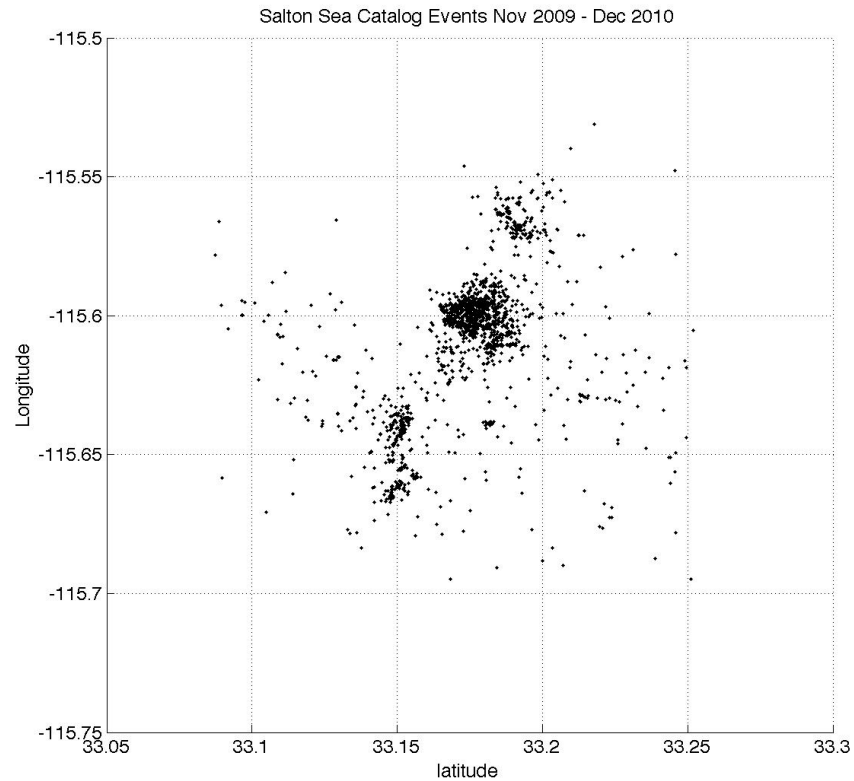

Figure 2. Map view of Salton Sea original catalog events between November 2009 and December 2010 plotted as black dots.

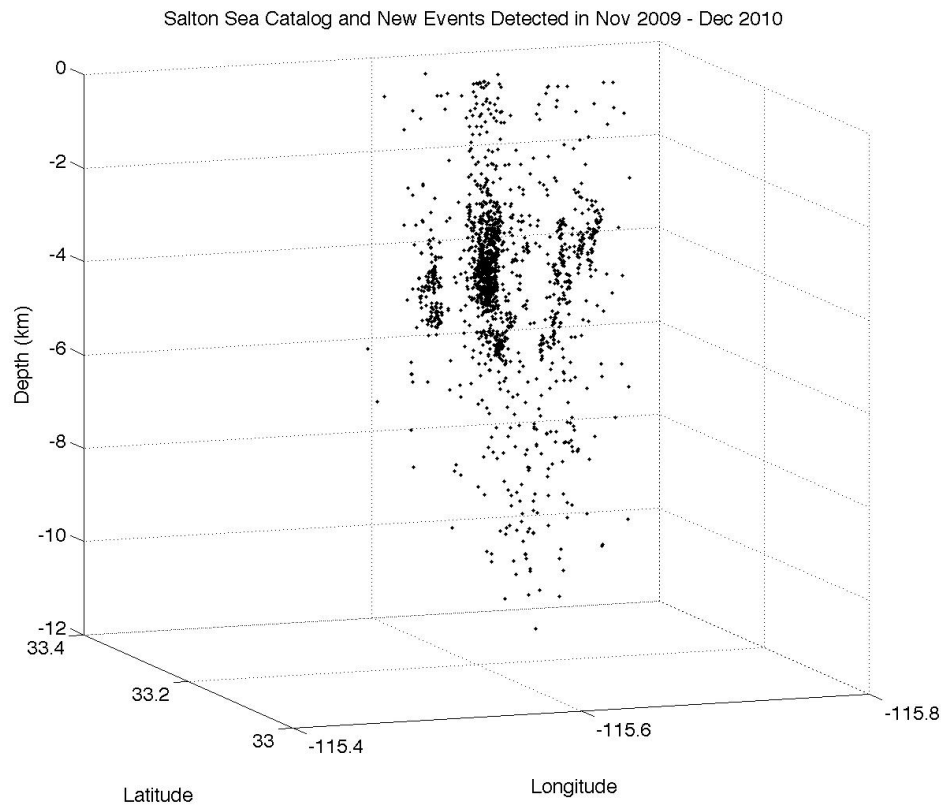

Figure 3. Depth view of Salton Sea original catalog events between November 2009 and December 2010 plotted as black dots. 


\subsection{Creation of Master Matching Templates}

We successfully created 231 master templates. Master templates are created from master events. The master events are selected based on two criteria. First, calibration events cannot be superimposed on other events on the seismic record. Second, waveforms of master events must also have high signal-to-noise ratios (SNR), especially in the lower frequency ranges, on at least four three-component seismic stations. No other selection criteria based on magnitude, mechanism or location is taken into consideration. Using these criteria, we investigated all catalog events occurring between 2008 and 2010 within $10 \mathrm{~km}$ of the station HAT for suitability as master events. We identify 231 events out of the original 2977 catalog events that occurred during this time frame that can be employed as master events (Appendix A). The distribution of master events relative to the entire 3-year catalog is shown in Figure 4.

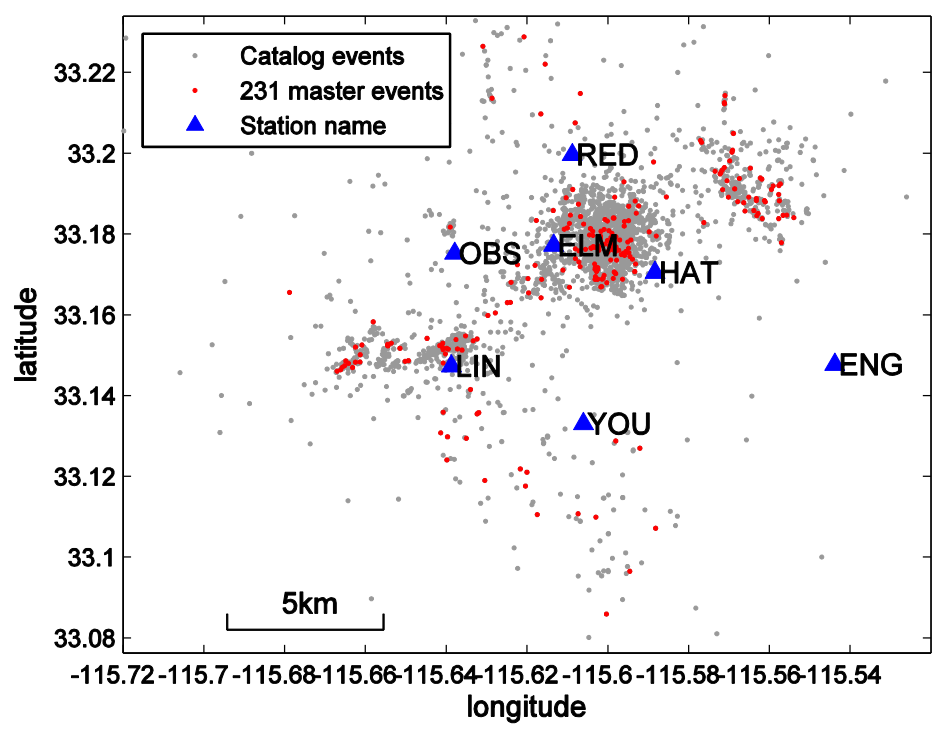

Figure 4. Map view of study area with EN seismic network indicated by blue triangles with station names. The grey dots are catalog events from SCEDC during three years from 2008 to 2010. The master templates are marked in red.

Figure 5 shows an example master event in the time and frequency domain. In general, the background noise is incoherent across the $70 \mathrm{sec}$ time window. However, station LIN displays a higher background noise level in the lower frequencies than the other stations. This was consistently true over all three years. As such, we removed station LIN from the processing due to its poor quality of recording. The other stations did not consistently display high background noise energy. 

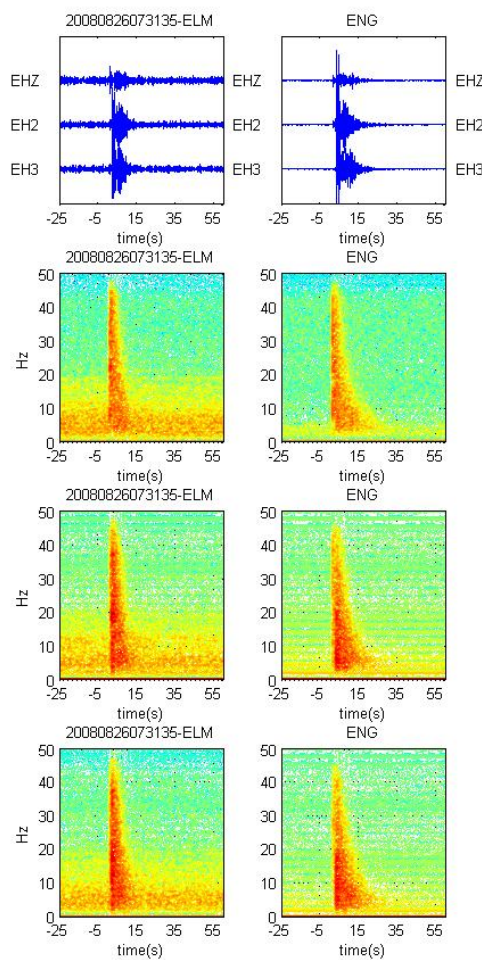

time(s)

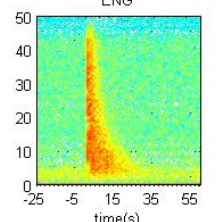

time(s)

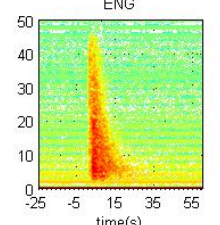

times(s)

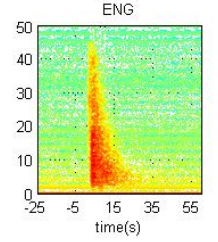

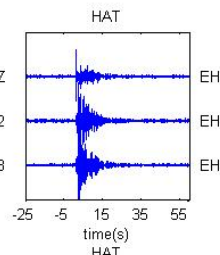

time(s)
HAT

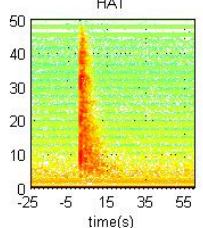

time(s)

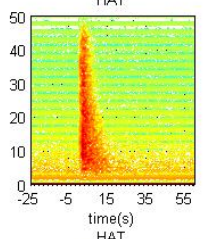

HAT

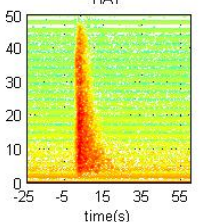

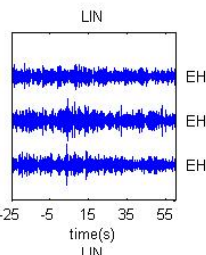

IN

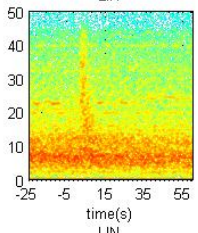

ime(s)

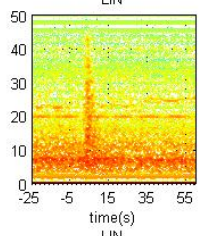

IN

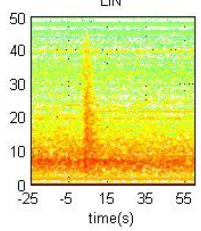

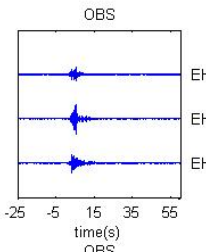

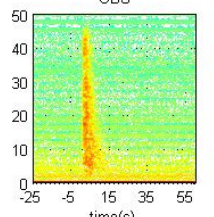

OBS

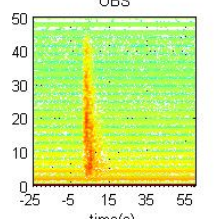

OBS

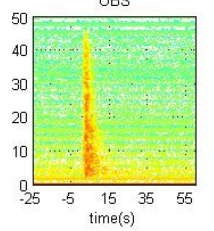

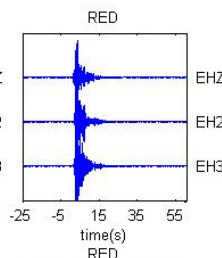

RED

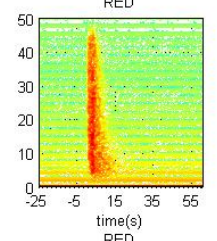

RED

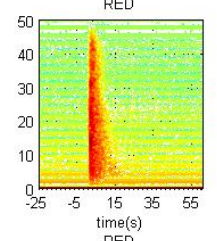

times $(s)$
RED

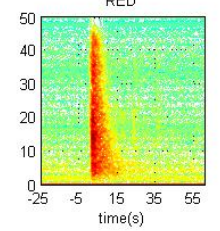

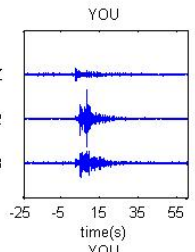

you

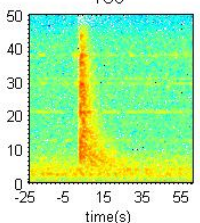

neu

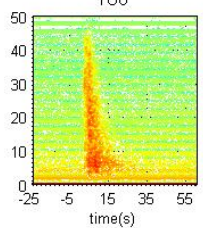

you

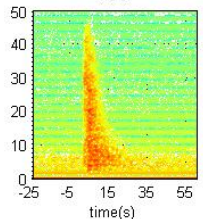

Figure 5. Example of a master event with origin time at August 26, 2008 07:31:35. The first row shows a plot of the waveform in the time domain. The station names are across top of each plot. The second, third and fourth rows show spectrograms of the EHZ, EH2 and EH3 components between $0-50 \mathrm{~Hz}$, respectively.

\subsection{Application of MFP Methodology to the Continuous Seismic Data}

For the SSGF test case, the empirical MFP code compared master templates to the continuous data using a 21 -sec sliding window that stepped forward at 1 -second intervals. We investigated three different frequency bands: $2-8 \mathrm{~Hz}, 4-10 \mathrm{~Hz}$, and $6-12 \mathrm{~Hz}$ to determine the best frequency band on which to apply empirical MFP.

Additionally, we augmented the basic detection algorithm to 1) remove triggers due to noise bursts and 2) large events outside of the study area. The first algorithm verifies that at least half of the observed components in the proposed detection contain signal energy in the $30-40 \mathrm{~Hz}$ range. This range was chosen since many proposed detections were being matched to relatively minor low frequency background noise also present in the master events.

The necessity of the second verification step became obvious after the nearby 4 April $2010 \mathrm{Mw}$ 7.2 El Mayor-Cucapah earthquake occurred. Many large aftershocks followed which were extremely well recorded across the EN seismic network over all frequencies. We speculate that the MFP algorithm was triggering on these events through a sidelobe of the aperture response function (analogous to the array response function for conventional beamforming). By looking at 
the data, it was obvious that these were not local events. We automatically excluded these larger known regional events by comparing potential detection origin times with the regional earthquake catalog.

\subsection{MFP Results}

\subsubsection{Comparison of different frequency bands}

A comparison between the number of real events and the number of initial detections that were subsequently removed after the spectrogram check for each of the three different frequency bands investigated shows that more real events are identified at the higher frequency ranges, however the number of potential detections that were ultimately removed increased significantly as well. The extra events identified at the higher frequency ranges tended to be extremely weak events or correlations with noise. Based on these results, we recommend the use of the $4-10 \mathrm{~Hz}$ frequency range for applications to the SSGF since this band detected a reasonably large number of events and balanced it with a reasonable number of events that were subsequently excluded during verification.

We believe that many of the matched field templates are corrupted by sinusoidal noise from rotating machinery in the geothermal field, or spatially structured wideband noise. We have observed that sinusoidal components in the wavefield cause an elevated detection statistic, as it is apparent that machinery (possibly pumps) turning on and off lead to sudden changes on the background detection statistic. In designing our MFP algorithm, we have assumed that the background noise is spatially white. Future research should be directed to dealing with a spatially structured noise environment.

\subsubsection{Comparison of different threshold levels}

We also select the optimal threshold level, which determines the strength of the detections found by the MFP method. We investigate thresholds ranging from 3 to 10. For example, a value of 3 indicates that the threshold level is set to 3 times of the average detection statistic averaged over a day.

\subsubsection{Detection of new events}

Comparing frequencies between $4-10 \mathrm{~Hz}$ and setting a threshold level of 3, we compared 231 master templates to the continuous data stream and identified 5357 local events (Figure 6 and Appendix B). The Hauksson et al. [2012] catalog reported 1536 events. 


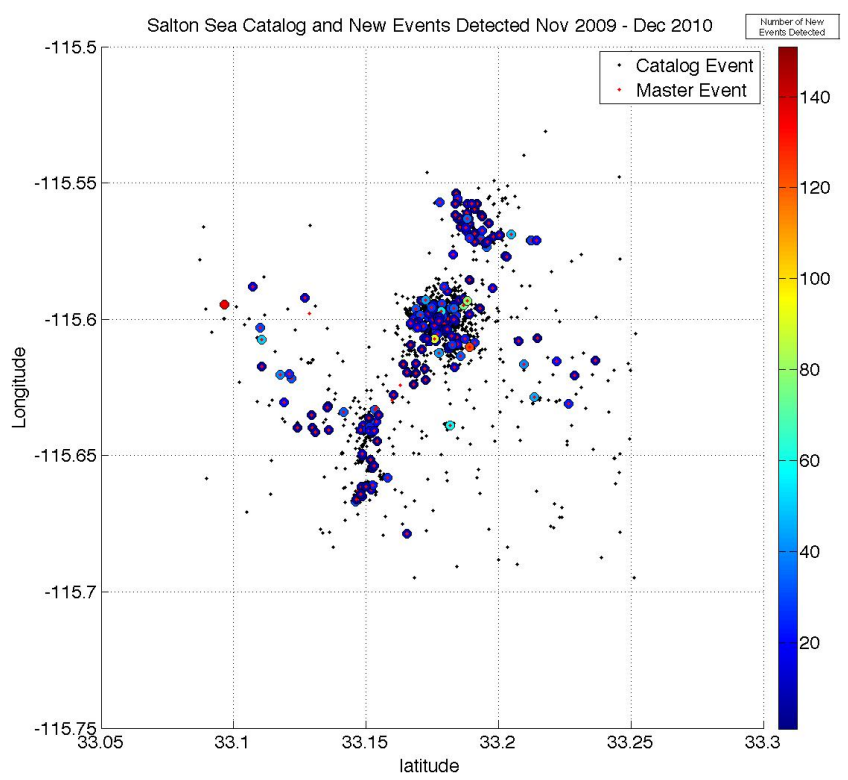

Figure 6. Plot in map view showing original catalog events as dots and the locations of the newly detected events as circles. The circles are color coded to indicate the number of new events the master event detected. Catalog events that were also designated as a master event, are plotted as a red dots.

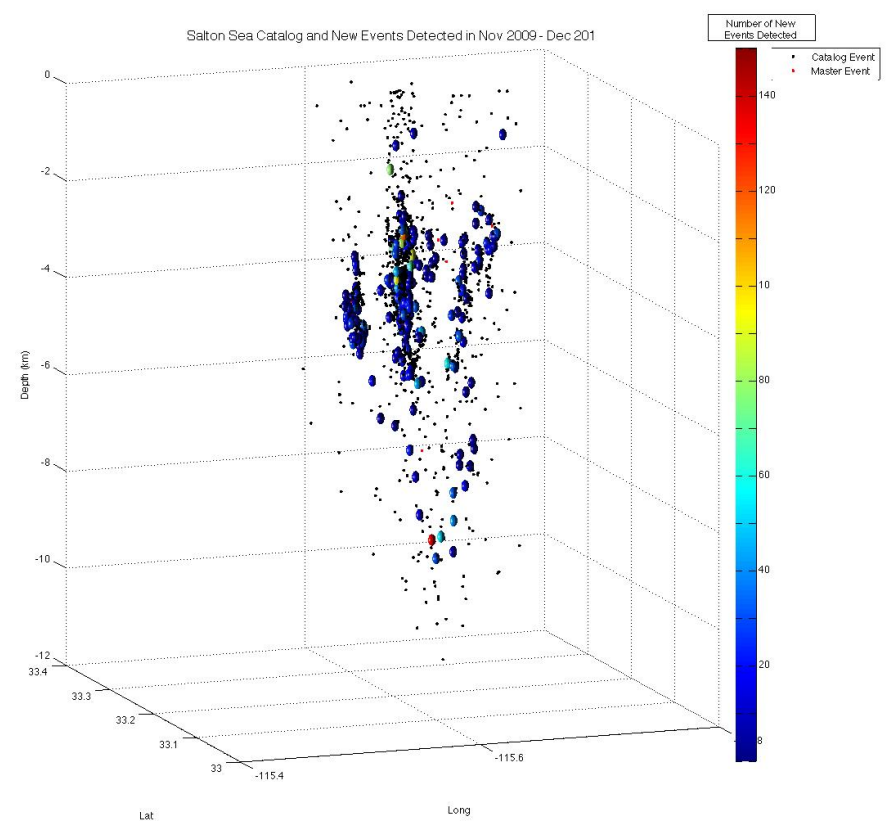

Figure 7. Plot in depth view showing original catalog events as dots and the locations of the newly detected events as circles. Notice that the three linear swarm regions in the shallower depths appear to have tails that come together to a single plane at deeper depths. 
Our method found 1235 of the 1536 catalog events reported in the Hauksson et al. [2012] catalog during the 14-month investigation period. There are $19.6 \%$ catalog events still missing. This is likely due to a lack of full coverage by master templates in the seismogenic region. Events in regions with no nearby master templates can be missed using the empirical MFP method. However, as more data become available with time, we can expect the coverage to improve. A list of the newly detected events can be found in Appendix B.

\subsubsection{Magnitude of new events}

We also investigated the magnitude of the new events in our study. Firstly, we define the SNR of the signal using

$$
S N R=\frac{\text { Amplitude of signal }- \text { Amplitude of noise }}{\text { Amplitude of noise }}
$$

We obtain the amplitude of the signal by stacking the $\mathrm{P}$ wave energy over all available vertical components. We determine the amplitude of noise by averaging the absolute value over 2 -second window right before the event origin time. In Figure 8, a regression line was drawn to obtain the empirical relationship in our region between the magnitude and $\log _{10}(\mathrm{SNR})$ of the catalog events. The newly detected events not reported by the Hauksson et al. [2012] catalog, are plotted along the regression line to obtain an estimated magnitude based on the above empirical relationship.

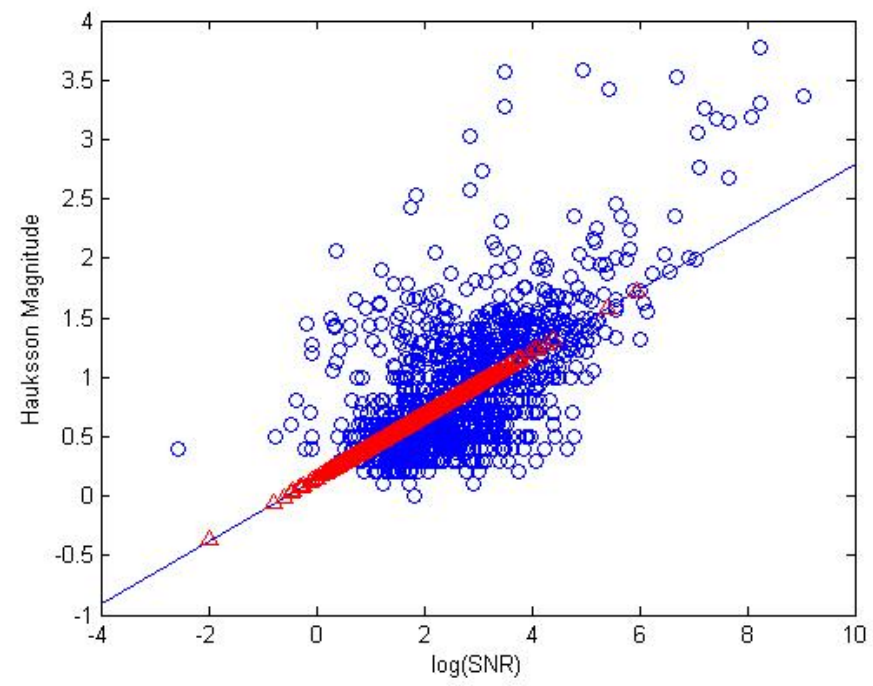

Figure 8. Regression relationship between the magnitude and $\log _{10}(S N R)$. The blue dots are the catalog events. The new events are plotted in red along the regression line. 
A histogram of events detected by both the Hauksson et al. [2012] catalog and the MFP method is plotted in Figure 9. The new events detected only by the MFP method are plotted in red and the catalog events are in blue. It is clear that the MFP method greatly increases the catalog completeness of the smaller magnitude events.

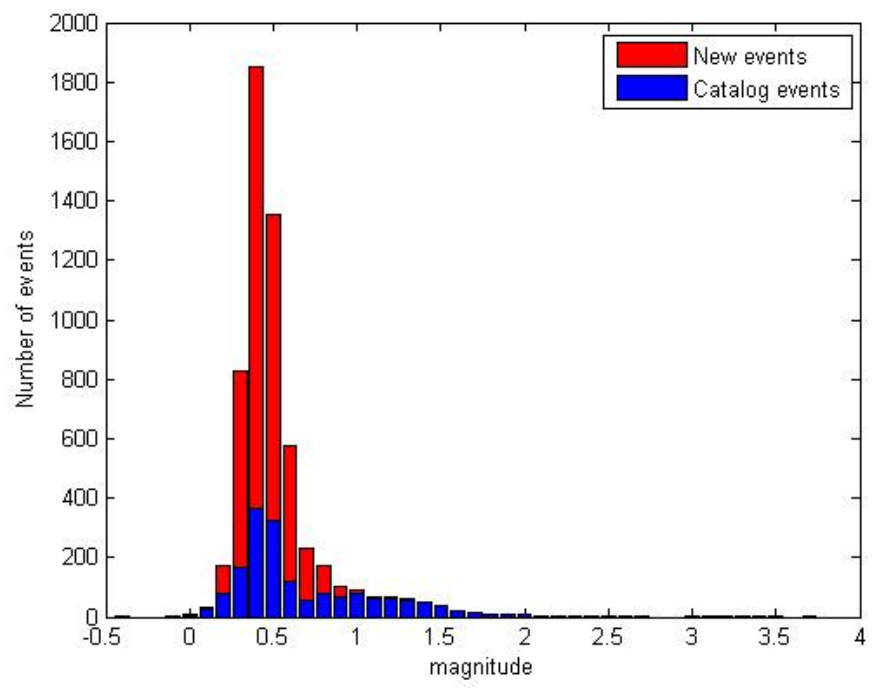

Figure 9. Histogram of the number of events versus the magnitude of the events. The red bars are events detected by the MFP method only. The blue bars are events detected by both MFP and the catalog.

\subsection{Conclusions}

MFP with empirically calibrated master templates is able to detect more events than can be detected using conventional techniques. Our test on the continuous data during November 2009 and December 2010 demonstrates the detection capability using the empirical MFP method. There are 5357 events detected in total by the new MFP method, while the catalog reports only 1536 events. A higher frequency band tends to find more weak events but at a higher false alarm rate. Consequently, we sought a suitable tradeoff between the number of real events and the number of false alarm by selecting $4-10 \mathrm{~Hz}$ and a threshold of 3 in this case study. These values may change in a different test location since they are dependent in part to the local noise structure. We believe that our method demonstrates that empirical MFP is a powerful tool to aid in the identification of more seismic events than those that can be identified and located using conventional event detection methods. 


\section{$4 \quad$ Salton Sea Geothermal Field Coulomb Stress Investigation}

\subsection{Static Stress Change Analysis and Results}

We calculate the static stress changes caused by the displacement of small faults using Coulomb3 [Lin et al. 2004; Toda et al. 2005]. Coulomb3 can calculate static displacements, strains, and stresses at any depth caused by fault slip, magmatic intrusion or dike expansion/contraction. We use the focal mechanism catalog of Yang et al. [2012] as input. We focus on the largest 6 events with M2 or greater occurring during the January 15, 2010 swarm to investigate the influence of these events on the overall sequence (Figures $8-12$ ). We also calculate the Coulomb stress change due to those ruptured faults and overlay the background seismicity between November 2009 and January 2010 in this region. We determined that most of the earthquakes occurred in the increased Coulomb stress regions caused by shear slip associated with the largest events.

\begin{tabular}{|rrrrrrrrrrrrrrrrrrr|r|r|r|}
\hline 2010 & 1 & 15 & 2 & 1 & 34.07 & 10526869 & 33.17417 & -115.599 & 3.91 & 3.05 & 322 & 88 & 142 & 27 & 39 & 15 & 0.14 & 32 & 0 B \\
\hline 2010 & 1 & 15 & 2 & 3 & 14.54 & 10526893 & 33.17484 & -115.594 & 4.39 & 2.25 & 318 & 77 & -153 & 41 & 37 & 14 & 0.11 & 2 & $2 \mathrm{C}$ \\
\hline 2010 & 1 & 15 & 2 & 12 & 56.49 & 10526957 & 33.17633 & -115.597 & 3.78 & 3.42 & 163 & 76 & -123 & 32 & 35 & 29 & 0.03 & 38 & $0 \mathrm{D}$ \\
\hline 2010 & 1 & 15 & 2 & 13 & 51.71 & 10526965 & 33.1725 & -115.599 & 3.9 & 3.52 & 162 & 86 & -124 & 21 & 26 & 20 & 0.21 & 15 & $1 \mathrm{~A}$ \\
\hline 2010 & 1 & 15 & 11 & 27 & 48.1 & 10528285 & 33.17817 & -115.601 & 4.45 & 2.04 & 201 & 76 & -96 & 23 & 22 & 23 & 0.24 & 25 & $1 \mathrm{~A}$ \\
\hline 2010 & 1 & 15 & 20 & 47 & 26.26 & 10529109 & 33.177 & -115.597 & 3.72 & 2.03 & 334 & 90 & 159 & 24 & 27 & 20 & 0.16 & 20 & $1 \mathrm{~B}$ \\
\hline
\end{tabular}

Table 1. Focal mechanism catalog information used in the Coulomb stress modeling by Yang et al. [2012]. The catalog format is in the order of: year, month, day, hour, minute and seconds, event ID, latitude and longitude, depth(km), magnitude, strike, dip, rake, fault plane uncertainty, number of $P$ wave first motions, misfit of first motions, number of $S / P$ amplitude ratios, average $\log _{10}(S / P$ amplitude ratio) misfit and quality of focal mechanism. 


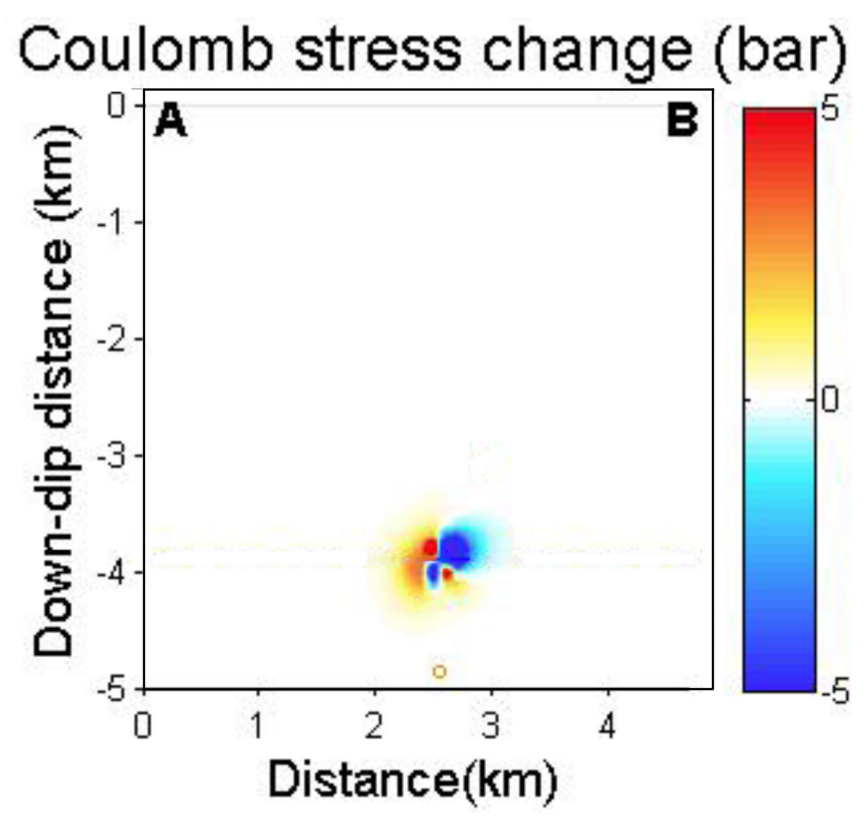

Figure 10. Cross section view of Coulomb stress change caused by the first event. The orange circle indicates the location of the next large event in the sequence. This shows that the first event should have been too small to influence the nucleation region of the next large event in the sequence.

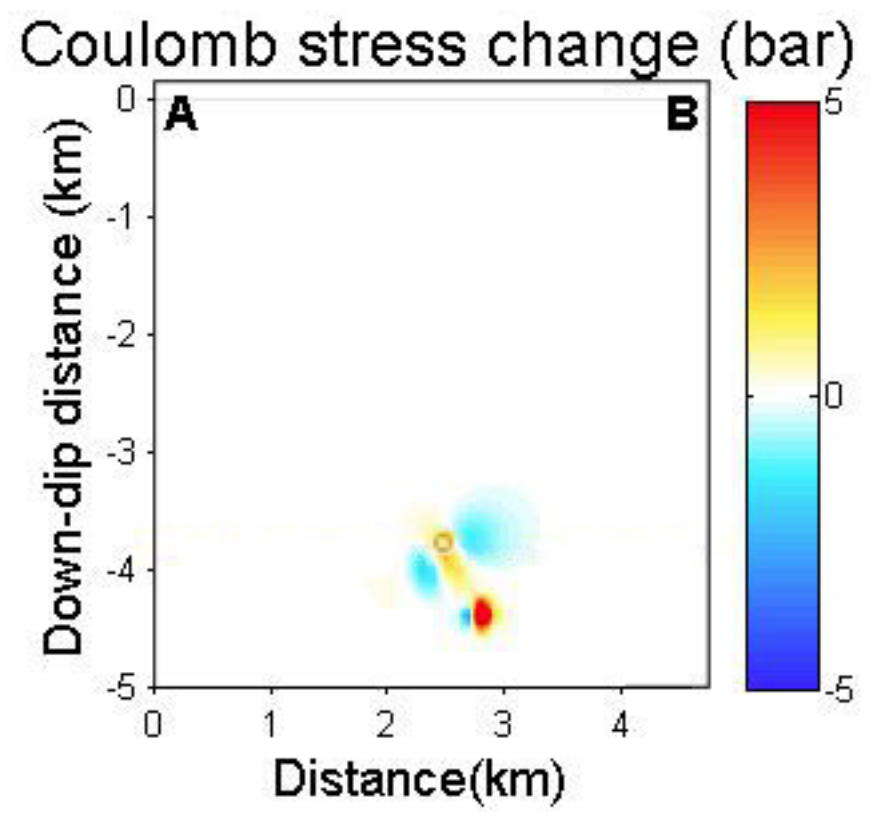

Figure 11. Cross section view of Coulomb stress changes caused by the first two events. The orange circle indicates the location of the next large event in the sequence. This plot shows that the next event did occur in a region of increased Coulomb stress. 


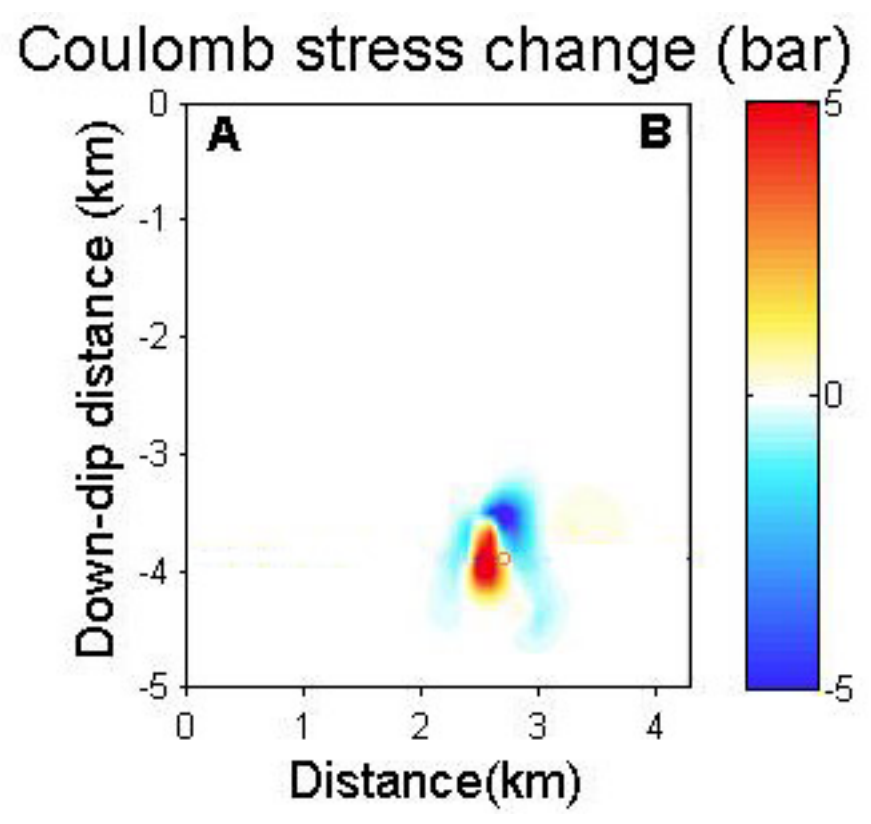

Figure 12. Cross section view of Coulomb stress changes caused by the first three events. The orange circle indicates the location of the next large event in the sequence. This plot shows that the next event did occur in a region of slightly increased Coulomb stress.

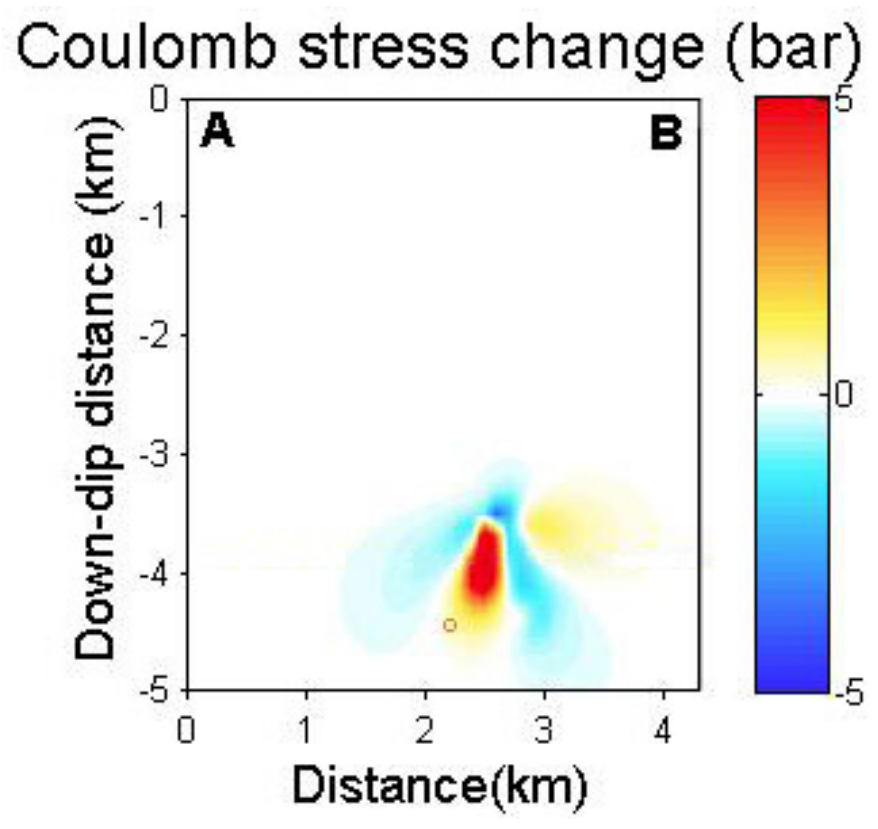

Figure 13. Cross section view of Coulomb stress changes caused by the first four events. The orange circle indicates the location of the next large event in the sequence. This plot shows that the next event did occur in a region of increased Coulomb stress. 


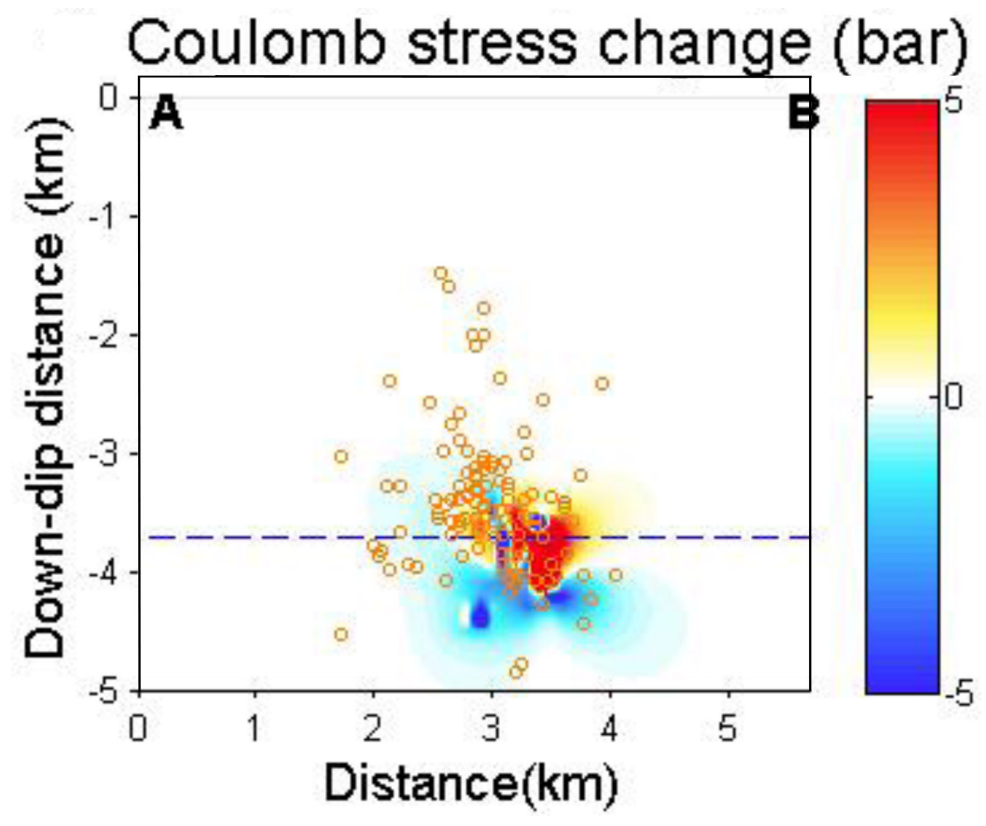

Figure 14. Cross section view of Coulomb stress changes caused by the largest six events with background seismicity as orange circles. The majority of the events occur in regions of increased Coulomb stress change, however this is somewhat difficult to view in 2D. See Figure 15 for a clearer plot covering a smaller time interval.

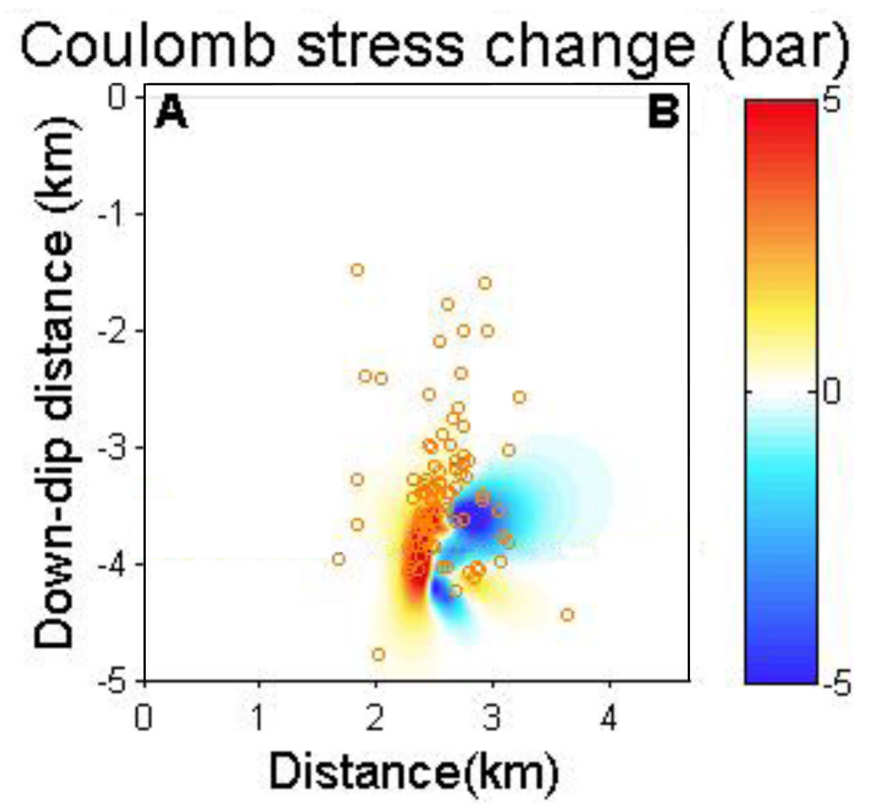

Figure 15. Cross section view of Coulomb stress changes caused by the middle three largest events with background seismicity during the same time period. The majority of the events occur in regions of increased Coulomb stress change. 


\section{$5 \quad$ Acknowledgements}

The facilities of the Southern California Earthquake Data Center (SCEDC), and the Southern California Seismic Network (SCSN), were used for access to waveforms, parametric data, and metadata required in this study.

The SCEDC and SCSN are funded through U.S. Geological Survey Grant G10AP00091, and the SCEDC, which is funded by NSF Cooperative Agreement EAR-0529922 and USGS Cooperative Agreement 07HQAG0008.

\section{References}

Ambuter, B.P., and Solomon, S.C., 1974. An event-recording system for monitoring small earthquakes, Bull. Seismol. Soc. Am., 64, 1181 - 1188.

Baggeroer, A.B., Kuperman,W.A. \&Mikhalevsky, P.N., 1993. An overview of matched field methods in ocean acoustics, IEEE Journal of Oceanic Engineering, 18(4), 401-424.

Bucker, H.P., 1976. Use of calculated sound fields and matched field detection to locate sound sources in shallow water, Journal of the Acoustical Society of America, 59(2), 368-373.

Fuis, G. S., and Mooney, W. D., 1990. Lithospheric structure of California along the San Andreas fault system, from seismic and other data, in Wallace, R. E., edit., The San Andreas Fault System: USGS Prof. Paper 1515, 207 - 236.

Fuis, G. S., Mooney, W. D., Healy, J. H., McMechan, G. A., and Lutter, W. J., 1984. A seismic refraction survey of the Imperial Valley region, California, J. Geophys. Res., 89, 1165 1189.

Gibbons, S.J. \& Ringdal, F., 2006. The detection of low magnitude seismic events using arraybased waveform correlation, Geophysical Journal International. 165, 149-166.

Gibbons, J. S., M. B. Sørensen, D. B. Harris, and F. Ringdal. (2007), The detection and location of low magnitude earthquakes in northern Norway using multi-channel waveform correlation at regional distances, Physics of The Earth and Planetary Interiors, v. 160, no. 3-4, 285-309.

Harris, D. B.., and T. Kvaerna, (2010), Superresolution with seismic arrays using empirical matched field processing, Geophysical Journal International, 182, 1455-1477. 
Harris, D. B. and D. A. Dodge (2011), An autonomous system for grouping events in a developing aftershock sequence, Bulletin of the Seismological Society of America, 101(1), pp. 763-774.

Hauksson, E. and W. Yang, and P.M. Shearer, "Waveform Relocated Earthquake Catalog for Southern California (1981 to 2011)"; Bull. Seismol. Soc. Am., Vol. 102, No. 5, pp.22392244, October 2012, doi: 10.1785/0120120010.

Lin, G., P. M. Shearer, and E. Hauksson (2007), Applying a three-dimensional velocity model, waveform cross correlation, and cluster analysis to locate southern California seismicity from 1981 to 2005, J. Geophys. Res., 112, B12309, doi: 10.1029/2007JB004986.

Lin, J., and R.S. Stein, Stress triggering in thrust and subduction earthquakes, and stress interaction between the southern San Andreas and nearby thrust and strike-slip faults, J. Geophys. Res., 109, B02303, doi:10.1029/2003JB002607, 2004.

Peng, Z., and P. Zhao. (2009), Migration of the Parkfield early aftershock sequence, Nature Geoscience, doi: 10.1038/ngeo697.

Portnoff, M. R. (1980), Time-frequency representation of digital signals and systems based on short-time Fourier analysis, IEEE Trans. on Acoustics, Speech and Signal Processing, ASSP28(1), 55-69.

Shelly, D. R., G. C. Beroza, S. Ide, and S. Nakamula (2006), Low-frequency earthquakes in Shikoku, Japan and their relationship to episodic tremor and slip, Nature, 442, 188-191.

Toda, S., R. S. Stein, K. Richards-Dinger and S. Bozkurt (2005), Forecasting the evolution of seismicity in southern California: Animations built on earthquake stress transfer, J. Geophys. Res., B05S16, doi:10.1029/2004JB003415. 


\section{Appendix A}

\section{Table of Master Events}

\begin{tabular}{|c|c|c|c|c|c|c|}
\hline Date & Time & Magnitude & Latitude & Longitude & Depth & EID \\
\hline $2008 / 01 / 21$ & 03:29:28.43 & 1.30 & 33.147 & -115.623 & 7.9 & 14343948 \\
\hline $2008 / 01 / 23$ & $18: 15: 57.18$ & 2.44 & 33.178 & -115.626 & 1.3 & 14344336 \\
\hline $2008 / 01 / 23$ & $20: 46: 08.50$ & 2.17 & 33.176 & -115.620 & 1.6 & 14344368 \\
\hline $2008 / 01 / 23$ & 21:43:09.04 & 1.44 & 33.170 & -115.626 & 0.6 & 14344388 \\
\hline $2008 / 03 / 13$ & $12: 46: 33.68$ & 2.53 & 33.149 & -115.640 & 3.5 & 10312161 \\
\hline $2008 / 03 / 15$ & 11:54:09.55 & 1.23 & 33.165 & -115.623 & 7.0 & 10312441 \\
\hline $2008 / 03 / 16$ & $08: 19: 22.43$ & 1.38 & 33.181 & -115.629 & 4.0 & 10312637 \\
\hline $2008 / 03 / 17$ & 17:09:37.31 & 1.22 & 33.186 & -115.624 & 2.5 & 10312869 \\
\hline $2008 / 03 / 19$ & 05:36:10.11 & 0.60 & 33.179 & -115.621 & 4.8 & 10313185 \\
\hline $2008 / 03 / 20$ & 09:40:02.73 & 1.73 & 33.190 & -115.608 & 2.2 & 10313381 \\
\hline $2008 / 03 / 21$ & 07:07:49.68 & 1.33 & 33.202 & -115.575 & 3.8 & 10313601 \\
\hline $2008 / 03 / 21$ & 07:19:17.54 & 1.15 & 33.189 & -115.619 & 2.2 & 10313605 \\
\hline $2008 / 04 / 02$ & $17: 24: 27.12$ & 0.80 & 33.188 & -115.613 & 2.5 & 14359568 \\
\hline $2008 / 04 / 09$ & $04: 19: 26.47$ & 1.32 & 33.193 & -115.632 & 3.5 & 10317021 \\
\hline $2008 / 04 / 11$ & $09: 10: 18.03$ & 1.26 & 33.203 & -115.561 & 3.0 & 10317505 \\
\hline $2008 / 04 / 11$ & 09:31:17.27 & 0.94 & 33.207 & -115.567 & 3.7 & 10317509 \\
\hline $2008 / 04 / 11$ & $11: 25: 28.31$ & 1.94 & 33.199 & -115.571 & 3.3 & 10317521 \\
\hline $2008 / 04 / 11$ & $11: 42: 53.76$ & 1.23 & 33.206 & -115.567 & 2.7 & 10317529 \\
\hline $2008 / 04 / 11$ & $23: 55: 23.51$ & 2.15 & 33.208 & -115.560 & 2.6 & 10317681 \\
\hline $2008 / 04 / 12$ & $00: 23: 10.79$ & 1.95 & 33.214 & -115.562 & 2.2 & 10317693 \\
\hline $2008 / 04 / 27$ & $07: 39: 32.65$ & 0.99 & 33.212 & -115.590 & 7.2 & 10320781 \\
\hline $2008 / 04 / 27$ & 09:49:19.12 & 1.46 & 33.229 & -115.576 & 3.4 & 10320805 \\
\hline $2008 / 04 / 27$ & $22: 12: 42.83$ & 1.08 & 33.226 & -115.588 & 4.6 & 10320941 \\
\hline $2008 / 05 / 13$ & 01:05:10.51 & 1.83 & 33.100 & -115.590 & 14.6 & 14368356 \\
\hline $2008 / 05 / 27$ & $11: 23: 03.53$ & 1.86 & 33.177 & -115.622 & 4.2 & 10326929 \\
\hline $2008 / 06 / 03$ & $05: 50: 30.63$ & 1.26 & 33.183 & -115.677 & 0.5 & 14372580 \\
\hline $2008 / 06 / 14$ & $23: 24: 42.28$ & 1.25 & 33.153 & -115.638 & 10.1 & 14375036 \\
\hline $2008 / 06 / 16$ & $07: 36: 57.46$ & 2.71 & 33.173 & -115.650 & 1.8 & 14375316 \\
\hline $2008 / 06 / 17$ & $08: 35: 15.42$ & 0.70 & 33.229 & -115.645 & 5.7 & 14375576 \\
\hline $2008 / 08 / 26$ & $07: 32: 35.85$ & 1.24 & 33.203 & -115.579 & 3.5 & 10345669 \\
\hline $2008 / 09 / 01$ & $14: 44: 57.65$ & 1.22 & 33.196 & -115.566 & 3.2 & 10346665 \\
\hline $2008 / 09 / 03$ & 18:04:01.76 & 3.25 & 33.189 & -115.567 & 2.5 & 10346905 \\
\hline $2008 / 09 / 06$ & $10: 15: 44.74$ & 1.06 & 33.157 & -115.668 & 0.1 & 10347337 \\
\hline $2008 / 09 / 30$ & $16: 21: 15.25$ & 1.60 & 33.157 & -115.656 & 2.4 & 14395952 \\
\hline $2008 / 10 / 06$ & $00: 40: 25.45$ & 1.20 & 33.159 & -115.635 & 7.3 & 10352673 \\
\hline $2008 / 10 / 08$ & $06: 18: 06.58$ & 0.40 & 33.181 & -115.665 & 4.2 & 10352985 \\
\hline $2008 / 10 / 12$ & 09:03:01.21 & 0.98 & 33.192 & -115.626 & 7.7 & 10353561 \\
\hline
\end{tabular}




\begin{tabular}{|c|c|c|c|c|c|c|}
\hline $2008 / 10 / 12$ & 15:03:11.60 & 1.50 & 33.197 & -115.614 & 5.3 & 10353629 \\
\hline $2008 / 10 / 14$ & $01: 36: 59.27$ & 1.39 & 33.231 & -115.655 & 4.8 & 10353853 \\
\hline $2008 / 10 / 23$ & $08: 47: 36.85$ & 1.47 & 33.140 & -115.636 & 7.3 & 10355457 \\
\hline $2008 / 10 / 25$ & $17: 49: 21.09$ & 1.01 & 33.231 & -115.605 & 4.8 & 10355901 \\
\hline $2008 / 10 / 30$ & $09: 21: 49.92$ & 1.21 & 33.201 & -115.584 & 3.7 & 10356973 \\
\hline $2008 / 10 / 31$ & 14:00:02.70 & 1.39 & 33.251 & -115.564 & 0.1 & 10357229 \\
\hline $2008 / 10 / 31$ & $14: 10: 32.20$ & 1.28 & 33.220 & -115.571 & 5.3 & 10357233 \\
\hline $2008 / 11 / 23$ & $20: 01: 25.29$ & 0.78 & 33.183 & -115.606 & 0.1 & 14405048 \\
\hline $2008 / 12 / 11$ & $23: 08: 12.67$ & 1.23 & 33.161 & -115.651 & 0.7 & 10365701 \\
\hline $2008 / 12 / 15$ & 12:06:33.82 & 1.07 & 33.165 & -115.638 & 0.1 & 10366549 \\
\hline $2008 / 12 / 24$ & $22: 48: 16.79$ & 1.05 & 33.158 & -115.638 & 0.4 & 10367949 \\
\hline $2008 / 12 / 31$ & 17:59:04.80 & 1.38 & 33.157 & -115.668 & 0.1 & 10368741 \\
\hline $2009 / 01 / 02$ & 01:00:30.48 & 1.40 & 33.118 & -115.590 & 13.8 & 10368981 \\
\hline $2009 / 01 / 03$ & $23: 18: 08.54$ & 1.43 & 33.135 & -115.644 & 6.9 & 10369313 \\
\hline $2009 / 01 / 07$ & 00:07:57.86 & 1.00 & 33.178 & -115.632 & 2.3 & 10369725 \\
\hline $2009 / 01 / 09$ & $04: 28: 52.91$ & 1.58 & 33.203 & -115.578 & 2.2 & 10370165 \\
\hline $2009 / 01 / 14$ & $11: 17: 40.04$ & 1.35 & 33.157 & -115.669 & 0.6 & 10371193 \\
\hline $2009 / 01 / 17$ & $03: 57: 23.81$ & 1.35 & 33.179 & -115.594 & 1.5 & 10371765 \\
\hline $2009 / 01 / 19$ & 09:53:14.21 & 1.23 & 33.241 & -115.633 & 3.4 & 10372145 \\
\hline $2009 / 01 / 30$ & 04:08:58.33 & 1.30 & 33.236 & -115.573 & 5.0 & 14418248 \\
\hline $2009 / 01 / 30$ & $08: 29: 29.22$ & 1.80 & 33.193 & -115.583 & 5.9 & 14418296 \\
\hline $2009 / 02 / 05$ & 07:56:06.89 & 1.28 & 33.197 & -115.659 & 6.4 & 14419456 \\
\hline $2009 / 02 / 07$ & 04:19:38.44 & 0.30 & 33.189 & -115.635 & 3.2 & 14419724 \\
\hline $2009 / 02 / 07$ & $07: 14: 31.32$ & 1.53 & 33.248 & -115.633 & 2.9 & 14419736 \\
\hline $2009 / 02 / 16$ & $03: 23: 10.48$ & 1.48 & 33.198 & -115.593 & 2.3 & 14421688 \\
\hline $2009 / 02 / 17$ & $18: 52: 15.46$ & 1.76 & 33.176 & -115.608 & 0.4 & 14422112 \\
\hline $2009 / 02 / 17$ & 18:58:00.31 & 1.51 & 33.176 & -115.604 & 0.5 & 14422120 \\
\hline $2009 / 02 / 19$ & $20: 54: 13.85$ & 1.17 & 33.180 & -115.600 & 0.1 & 14422896 \\
\hline $2009 / 02 / 20$ & $14: 19: 27.03$ & 0.30 & 33.214 & -115.620 & 5.3 & 10377717 \\
\hline $2009 / 02 / 21$ & $05: 58: 22.03$ & 0.50 & 33.158 & -115.629 & 6.0 & 10377881 \\
\hline $2009 / 03 / 01$ & $22: 21: 40.52$ & 0.80 & 33.170 & -115.654 & 0.0 & 10379337 \\
\hline $2009 / 03 / 02$ & $14: 02: 48.42$ & 1.89 & 33.186 & -115.612 & 0.6 & 10379437 \\
\hline $2009 / 03 / 03$ & $12: 15: 27.26$ & 1.79 & 33.197 & -115.596 & 3.0 & 10379553 \\
\hline $2009 / 03 / 03$ & $12: 45: 09.38$ & 0.50 & 33.234 & -115.604 & 4.8 & 10379561 \\
\hline $2009 / 03 / 03$ & $18: 00: 40.28$ & 0.30 & 33.221 & -115.605 & 5.1 & 10379605 \\
\hline $2009 / 03 / 15$ & $14: 10: 15.23$ & 1.30 & 33.208 & -115.631 & 5.0 & 14430104 \\
\hline $2009 / 03 / 16$ & 08:59:11.51 & 1.48 & 33.189 & -115.605 & 1.5 & 14430344 \\
\hline $2009 / 03 / 21$ & 08:54:07.31 & 1.27 & 33.212 & -115.633 & 7.6 & 14432232 \\
\hline $2009 / 03 / 24$ & 18:59:11.89 & 0.90 & 33.188 & -115.599 & 0.8 & 14434048 \\
\hline $2009 / 03 / 31$ & $17: 41: 12.68$ & 0.90 & 33.199 & -115.570 & 0.2 & 14439288 \\
\hline $2009 / 04 / 01$ & $04: 38: 58.48$ & 2.11 & 33.181 & -115.612 & 1.1 & 14439632 \\
\hline $2009 / 04 / 01$ & $23: 56: 27.90$ & 1.18 & 33.181 & -115.614 & 3.1 & 14439984 \\
\hline $2009 / 04 / 03$ & 02:56:02.70 & 1.44 & 33.159 & -115.650 & 1.5 & 14440432 \\
\hline $2009 / 04 / 07$ & $09: 45: 29.39$ & 1.31 & 33.184 & -115.611 & 2.7 & 14441880 \\
\hline
\end{tabular}




\begin{tabular}{|c|c|c|c|c|c|c|}
\hline $2009 / 04 / 15$ & 09:53:41.73 & 1.58 & 33.157 & -115.680 & 0.2 & 14444520 \\
\hline $2009 / 04 / 15$ & 09:56:05.85 & 1.71 & 33.155 & -115.663 & 2.3 & 14444528 \\
\hline $2009 / 04 / 15$ & $10: 21: 37.44$ & 0.80 & 33.185 & -115.687 & 7.0 & 14444544 \\
\hline $2009 / 04 / 17$ & $04: 35: 34.83$ & 2.72 & 33.182 & -115.602 & 2.5 & 10396569 \\
\hline $2009 / 04 / 19$ & $15: 30: 07.85$ & 0.90 & 33.158 & -115.650 & 0.0 & 10397553 \\
\hline $2009 / 04 / 21$ & $03: 59: 40.46$ & 0.85 & 33.189 & -115.576 & 0.1 & 10398241 \\
\hline $2009 / 04 / 25$ & $17: 19: 50.41$ & 1.63 & 33.160 & -115.633 & 4.6 & 10400553 \\
\hline $2009 / 05 / 05$ & $10: 40: 16.23$ & 1.10 & 33.166 & -115.617 & 5.3 & 10405193 \\
\hline $2009 / 05 / 06$ & $14: 11: 13.12$ & 0.55 & 33.202 & -115.671 & 6.3 & 10405657 \\
\hline $2009 / 05 / 11$ & $19: 52: 28.34$ & 1.49 & 33.196 & -115.587 & 4.5 & 10407569 \\
\hline $2009 / 05 / 11$ & $20: 17: 21.73$ & 1.20 & 33.176 & -115.606 & 6.3 & 10407577 \\
\hline $2009 / 05 / 15$ & $14: 07: 57.62$ & 0.50 & 33.154 & -115.664 & 0.1 & 10409217 \\
\hline $2009 / 05 / 28$ & 01:26:10.01 & 2.47 & 33.184 & -115.609 & 2.2 & 14464200 \\
\hline $2009 / 05 / 28$ & $03: 10: 29.29$ & 0.30 & 33.196 & -115.587 & 5.3 & 14464264 \\
\hline $2009 / 06 / 11$ & $04: 30: 50.36$ & 0.50 & 33.196 & -115.618 & 7.3 & 14471936 \\
\hline $2009 / 06 / 17$ & $14: 38: 30.48$ & 1.28 & 33.192 & -115.579 & 4.3 & 14475632 \\
\hline $2009 / 06 / 21$ & $14: 40: 23.06$ & 1.57 & 33.176 & -115.609 & 2.1 & 14477824 \\
\hline $2009 / 07 / 02$ & $19: 17: 37.06$ & 1.29 & 33.160 & -115.642 & 1.5 & 10433953 \\
\hline $2009 / 07 / 09$ & $12: 15: 41.29$ & 0.45 & 33.197 & -115.644 & 3.0 & 10436601 \\
\hline $2009 / 07 / 18$ & $12: 16: 57.19$ & 1.45 & 33.139 & -115.602 & 7.0 & 10439825 \\
\hline $2009 / 07 / 30$ & 09:39:54.46 & 1.79 & 33.190 & -115.612 & 1.0 & 14489908 \\
\hline $2009 / 07 / 31$ & 04:13:01.69 & 0.80 & 33.193 & -115.602 & 0.0 & 14493288 \\
\hline $2009 / 08 / 01$ & $11: 30: 54.47$ & 0.60 & 33.154 & -115.669 & 0.6 & 14494096 \\
\hline $2009 / 08 / 01$ & $12: 38: 22.64$ & 1.51 & 33.179 & -115.614 & 1.8 & 14494112 \\
\hline $2009 / 08 / 01$ & $13: 04: 50.59$ & 1.42 & 33.184 & -115.608 & 0.3 & 14494136 \\
\hline $2009 / 08 / 02$ & $15: 46: 26.12$ & 0.30 & 33.187 & -115.622 & 4.0 & 14494600 \\
\hline $2009 / 08 / 03$ & $06: 47: 08.19$ & 1.47 & 33.187 & -115.601 & 1.7 & 14495024 \\
\hline $2009 / 08 / 04$ & 01:11:08.19 & 0.30 & 33.191 & -115.684 & 3.3 & 14495328 \\
\hline $2009 / 08 / 11$ & $08: 36: 39.45$ & 1.74 & 33.142 & -115.628 & 10.3 & 14498800 \\
\hline $2009 / 09 / 18$ & 08:15:34.86 & 0.95 & 33.159 & -115.641 & 0.9 & 14511836 \\
\hline $2009 / 10 / 02$ & 23:01:58.64 & 1.46 & 33.164 & -115.640 & 3.4 & 14519604 \\
\hline $2009 / 10 / 03$ & 13:21:05.01 & 1.25 & 33.186 & -115.604 & 2.0 & 14521004 \\
\hline $2009 / 10 / 07$ & $07: 13: 33.78$ & 1.32 & 33.181 & -115.659 & 6.0 & 14524084 \\
\hline $2009 / 10 / 08$ & $23: 18: 33.72$ & 2.05 & 33.185 & -115.569 & 3.7 & 10477781 \\
\hline 2009/10/09 & $00: 25: 33.17$ & 1.31 & 33.188 & -115.571 & 3.0 & 10477837 \\
\hline $2009 / 10 / 11$ & $17: 37: 31.10$ & 1.22 & 33.201 & -115.544 & 3.4 & 10479509 \\
\hline $2009 / 10 / 21$ & 16:02:05.89 & 0.75 & 33.209 & -115.590 & 3.1 & 10483853 \\
\hline $2009 / 10 / 30$ & $12: 35: 26.61$ & 1.44 & 33.157 & -115.648 & 0.1 & 10487285 \\
\hline $2009 / 11 / 01$ & 20:46:52.49 & 2.39 & 33.165 & -115.643 & 1.7 & 10488533 \\
\hline $2009 / 11 / 10$ & $12: 22: 04.49$ & 1.06 & 33.181 & -115.611 & 0.4 & 10495013 \\
\hline $2009 / 11 / 10$ & $12: 34: 34.87$ & 1.32 & 33.181 & -115.608 & 1.0 & 10495021 \\
\hline $2009 / 11 / 16$ & $11: 29: 38.33$ & 0.70 & 33.158 & -115.642 & 0.1 & 10497589 \\
\hline $2009 / 11 / 17$ & $19: 29: 46.46$ & 0.00 & 33.188 & -115.573 & 0.2 & 10498285 \\
\hline $2009 / 11 / 17$ & $20: 30: 49.13$ & 1.24 & 33.170 & -115.600 & 1.1 & 10498349 \\
\hline
\end{tabular}




\begin{tabular}{|c|c|c|c|c|c|c|}
\hline $2009 / 11 / 18$ & 03:02:32.41 & 0.50 & 33.173 & -115.609 & 0.1 & 14546172 \\
\hline $2009 / 11 / 18$ & $07: 28: 24.18$ & 0.94 & 33.173 & -115.610 & 1.3 & 14546220 \\
\hline $2009 / 11 / 18$ & $07: 45: 29.15$ & 0.70 & 33.172 & -115.609 & 0.1 & 14546228 \\
\hline $2009 / 11 / 18$ & $10: 19: 48.14$ & 1.25 & 33.182 & -115.606 & 1.7 & 14546276 \\
\hline $2009 / 11 / 18$ & $11: 57: 44.95$ & 0.30 & 33.171 & -115.605 & 0.7 & 14546420 \\
\hline $2009 / 11 / 18$ & $13: 02: 03.16$ & 0.40 & 33.186 & -115.614 & 2.4 & 14546452 \\
\hline $2009 / 11 / 18$ & $13: 45: 32.87$ & 0.50 & 33.178 & -115.611 & 1.6 & 14546468 \\
\hline $2009 / 11 / 18$ & $17: 47: 39.00$ & 1.18 & 33.172 & -115.610 & 2.2 & 14546572 \\
\hline $2009 / 11 / 19$ & 09:30:23.85 & 0.70 & 33.184 & -115.605 & 0.1 & 10141762 \\
\hline $2009 / 11 / 24$ & $12: 20: 54.66$ & 1.01 & 33.182 & -115.618 & 3.1 & 14549044 \\
\hline $2009 / 11 / 24$ & $13: 26: 53.81$ & 1.31 & 33.181 & -115.602 & 0.4 & 14549100 \\
\hline $2009 / 11 / 25$ & $00: 47: 50.90$ & 1.56 & 33.157 & -115.641 & 2.2 & 14549556 \\
\hline $2009 / 11 / 25$ & $13: 51: 56.03$ & 0.50 & 33.185 & -115.608 & 2.0 & 14549908 \\
\hline $2009 / 11 / 27$ & $15: 25: 43.54$ & 0.60 & 33.199 & -115.617 & 0.0 & 14551420 \\
\hline $2009 / 11 / 29$ & $06: 44: 47.88$ & 1.66 & 33.195 & -115.572 & 0.1 & 14551996 \\
\hline $2009 / 11 / 30$ & 11:42:05.01 & 1.00 & 33.187 & -115.604 & 3.2 & 14552468 \\
\hline $2009 / 12 / 03$ & 21:09:16.44 & 1.28 & 33.183 & -115.607 & 0.0 & 10507373 \\
\hline $2009 / 12 / 04$ & $13: 56: 39.01$ & 1.44 & 33.190 & -115.604 & 1.2 & 14554428 \\
\hline $2009 / 12 / 08$ & 03:02:46.99 & 1.34 & 33.213 & -115.584 & 1.3 & 14555660 \\
\hline $2009 / 12 / 08$ & $13: 11: 52.26$ & 1.68 & 33.159 & -115.640 & 1.5 & 14555772 \\
\hline $2009 / 12 / 11$ & $10: 49: 17.44$ & 1.01 & 33.196 & -115.559 & 0.2 & 14557228 \\
\hline $2009 / 12 / 28$ & $16: 29: 42.29$ & 1.46 & 33.181 & -115.610 & 0.9 & 14564844 \\
\hline $2009 / 12 / 31$ & 09:03:22.57 & 1.00 & 33.186 & -115.593 & 0.1 & 14566380 \\
\hline $2010 / 01 / 06$ & $03: 44: 48.05$ & 1.28 & 33.136 & -115.649 & 5.3 & 14569172 \\
\hline $2010 / 01 / 10$ & $23: 50: 57.28$ & 0.50 & 33.175 & -115.600 & 1.1 & 14571348 \\
\hline $2010 / 01 / 11$ & $00: 25: 36.28$ & 0.90 & 33.179 & -115.597 & 0.1 & 14571372 \\
\hline $2010 / 01 / 13$ & $14: 14: 30.30$ & 0.40 & 33.175 & -115.610 & 2.4 & 10525845 \\
\hline $2010 / 01 / 13$ & $16: 40: 24.77$ & 1.36 & 33.176 & -115.602 & 3.2 & 10525909 \\
\hline $2010 / 01 / 15$ & 08:06:14.40 & 1.07 & 33.188 & -115.598 & 2.3 & 10527749 \\
\hline $2010 / 01 / 15$ & 08:12:53.81 & 0.91 & 33.180 & -115.603 & 0.1 & 10527765 \\
\hline $2010 / 01 / 15$ & $08: 42: 51.12$ & 0.70 & 33.182 & -115.603 & 0.1 & 10527845 \\
\hline $2010 / 01 / 15$ & $08: 51: 24.36$ & 0.91 & 33.188 & -115.609 & 2.3 & 10527917 \\
\hline $2010 / 01 / 15$ & $16: 43: 18.01$ & 0.98 & 33.184 & -115.604 & 0.1 & 10528797 \\
\hline $2010 / 01 / 15$ & $23: 53: 46.60$ & 1.49 & 33.181 & -115.603 & 2.0 & 10529317 \\
\hline $2010 / 01 / 16$ & $01: 24: 47.48$ & 1.32 & 33.188 & -115.600 & 0.1 & 10529381 \\
\hline $2010 / 01 / 16$ & 01:26:20.69 & 1.23 & 33.182 & -115.612 & 2.2 & 10529389 \\
\hline $2010 / 01 / 16$ & $21: 10: 23.18$ & 1.22 & 33.173 & -115.617 & 0.2 & 10530381 \\
\hline $2010 / 01 / 19$ & 09:51:03.82 & 0.80 & 33.181 & -115.601 & 0.7 & 10532053 \\
\hline $2010 / 01 / 19$ & $18: 28: 48.50$ & 0.70 & 33.184 & -115.610 & 2.8 & 10532245 \\
\hline $2010 / 01 / 21$ & $19: 19: 36.22$ & 0.50 & 33.172 & -115.622 & 4.0 & 10532861 \\
\hline $2010 / 01 / 22$ & 09:41:54.10 & 1.21 & 33.161 & -115.644 & 1.0 & 10533069 \\
\hline $2010 / 01 / 22$ & $10: 12: 40.72$ & 1.00 & 33.161 & -115.644 & 0.3 & 10533085 \\
\hline $2010 / 01 / 22$ & $11: 06: 33.10$ & 1.69 & 33.165 & -115.649 & 1.4 & 10533093 \\
\hline $2010 / 01 / 22$ & $17: 08: 15.35$ & 1.41 & 33.145 & -115.634 & 4.8 & 10533173 \\
\hline
\end{tabular}




\begin{tabular}{|c|c|c|c|c|c|c|}
\hline $2010 / 01 / 22$ & $17: 09: 30.35$ & 1.34 & 33.148 & -115.633 & 4.3 & 10533181 \\
\hline $2010 / 01 / 23$ & $13: 51: 11.52$ & 0.93 & 33.161 & -115.640 & 0.1 & 10533613 \\
\hline $2010 / 01 / 23$ & 15:38:13.31 & 0.90 & 33.166 & -115.642 & 2.3 & 10533701 \\
\hline $2010 / 01 / 24$ & 01:18:13.41 & 0.50 & 33.158 & -115.644 & 0.1 & 10533909 \\
\hline $2010 / 01 / 24$ & $20: 12: 53.03$ & 0.40 & 33.163 & -115.637 & 0.1 & 10534069 \\
\hline $2010 / 01 / 28$ & $18: 26: 57.69$ & 1.08 & 33.196 & -115.573 & 0.1 & 10535733 \\
\hline $2010 / 01 / 28$ & $18: 32: 13.21$ & 0.60 & 33.198 & -115.571 & 0.2 & 10535781 \\
\hline $2010 / 01 / 29$ & $07: 37: 28.18$ & 0.63 & 33.174 & -115.603 & 0.1 & 10535973 \\
\hline $2010 / 01 / 29$ & 19:05:33.58 & 1.49 & 33.176 & -115.618 & 3.7 & 10536197 \\
\hline $2010 / 01 / 30$ & $18: 49: 36.40$ & 1.00 & 33.212 & -115.610 & 4.1 & 10536445 \\
\hline $2010 / 02 / 02$ & $19: 15: 14.45$ & 0.70 & 33.176 & -115.604 & 0.0 & 14584564 \\
\hline $2010 / 02 / 07$ & $09: 42: 50.68$ & 1.48 & 33.177 & -115.607 & 4.2 & 14586364 \\
\hline $2010 / 02 / 11$ & $03: 20: 49.27$ & 1.00 & 33.168 & -115.609 & 0.7 & 10541045 \\
\hline $2010 / 02 / 12$ & $15: 24: 15.96$ & 1.11 & 33.188 & -115.573 & 1.2 & 10541541 \\
\hline $2010 / 02 / 19$ & $21: 31: 47.28$ & 0.50 & 33.181 & -115.603 & 0.1 & 10545045 \\
\hline $2010 / 03 / 03$ & $15: 17: 42.70$ & 1.47 & 33.208 & -115.582 & 2.5 & 14596596 \\
\hline $2010 / 03 / 04$ & $10: 01: 19.47$ & 1.48 & 33.184 & -115.606 & 2.9 & 14596868 \\
\hline $2010 / 03 / 04$ & $16: 47: 58.88$ & 1.10 & 33.176 & -115.609 & 3.8 & 14596900 \\
\hline $2010 / 03 / 07$ & $19: 37: 35.80$ & 1.29 & 33.130 & -115.634 & 0.0 & 14597756 \\
\hline $2010 / 03 / 08$ & $21: 28: 41.61$ & 0.60 & 33.177 & -115.635 & 5.3 & 14598092 \\
\hline $2010 / 03 / 21$ & $02: 36: 12.03$ & 0.73 & 33.175 & -115.606 & 4.2 & 14602892 \\
\hline $2010 / 06 / 23$ & $12: 39: 31.00$ & 0.70 & 33.181 & -115.608 & 0.0 & 10721421 \\
\hline $2010 / 06 / 26$ & $00: 26: 48.34$ & 0.70 & 33.179 & -115.602 & 0.6 & 10725013 \\
\hline $2010 / 07 / 09$ & 10:09:12.33 & 2.05 & 33.192 & -115.567 & 3.1 & 10743997 \\
\hline $2010 / 07 / 09$ & $10: 10: 52.05$ & 1.40 & 33.194 & -115.567 & 3.1 & 10744005 \\
\hline $2010 / 07 / 13$ & $15: 13: 57.41$ & 1.76 & 33.118 & -115.610 & 9.1 & 10750725 \\
\hline $2010 / 08 / 03$ & $19: 35: 41.56$ & 1.87 & 33.183 & -115.617 & 4.3 & 10769261 \\
\hline $2010 / 08 / 05$ & $01: 45: 48.00$ & 1.00 & 33.183 & -115.599 & 0.1 & 10770181 \\
\hline $2010 / 08 / 05$ & $23: 24: 26.43$ & 1.29 & 33.181 & -115.639 & 3.7 & 10770741 \\
\hline $2010 / 08 / 06$ & $13: 10: 14.05$ & 0.40 & 33.175 & -115.601 & 0.1 & 10771453 \\
\hline $2010 / 08 / 13$ & $23: 15: 36.76$ & 1.70 & 33.194 & -115.583 & 3.0 & 10777933 \\
\hline $2010 / 08 / 14$ & $02: 49: 40.56$ & 1.59 & 33.198 & -115.586 & 3.7 & 10778029 \\
\hline $2010 / 08 / 14$ & 04:39:15.40 & 1.12 & 33.200 & -115.590 & 3.5 & 10778069 \\
\hline $2010 / 08 / 14$ & $15: 23: 18.62$ & 0.70 & 33.190 & -115.574 & 3.1 & 10778461 \\
\hline $2010 / 08 / 14$ & $15: 24: 52.21$ & 1.19 & 33.192 & -115.572 & 1.6 & 10778469 \\
\hline $2010 / 08 / 17$ & 01:49:10.31 & 0.60 & 33.197 & -115.577 & 0.1 & 10780213 \\
\hline $2010 / 08 / 18$ & 09:14:43.34 & 1.16 & 33.141 & -115.641 & 4.5 & 10781237 \\
\hline $2010 / 08 / 21$ & $22: 46: 49.14$ & 0.60 & 33.177 & -115.606 & 0.1 & 10783877 \\
\hline $2010 / 08 / 28$ & $07: 58: 25.17$ & 1.43 & 33.218 & -115.639 & 3.0 & 10787821 \\
\hline $2010 / 08 / 29$ & $10: 51: 02.02$ & 0.91 & 33.231 & -115.657 & 2.0 & 10788677 \\
\hline $2010 / 09 / 04$ & $08: 26: 30.75$ & 1.67 & 33.115 & -115.585 & 6.5 & 10792285 \\
\hline $2010 / 09 / 06$ & $11: 10: 30.80$ & 1.36 & 33.154 & -115.672 & 0.3 & 10793533 \\
\hline $2010 / 09 / 17$ & $21: 44: 18.15$ & 0.40 & 33.174 & -115.604 & 0.1 & 14842828 \\
\hline $2010 / 09 / 17$ & $21: 51: 52.12$ & 2.01 & 33.177 & -115.614 & 1.2 & 14842852 \\
\hline
\end{tabular}




\begin{tabular}{|l|l|l|l|l|l|l|}
\hline $2010 / 09 / 29$ & $02: 09: 42.16$ & 1.55 & 33.228 & -115.626 & 3.7 & 14849452 \\
\hline $2010 / 11 / 11$ & $19: 53: 46.34$ & 0.70 & 33.180 & -115.609 & 0.0 & 10837357 \\
\hline $2010 / 11 / 14$ & $04: 59: 13.35$ & 1.00 & 33.181 & -115.603 & 1.4 & 10838725 \\
\hline $2010 / 11 / 14$ & $16: 50: 40.20$ & 1.48 & 33.164 & -115.632 & 2.6 & 10838925 \\
\hline $2010 / 11 / 17$ & $15: 10: 06.33$ & 0.80 & 33.184 & -115.601 & 3.5 & 10840661 \\
\hline $2010 / 11 / 26$ & $13: 01: 28.39$ & 0.70 & 33.164 & -115.668 & 0.0 & 14886772 \\
\hline $2010 / 12 / 03$ & $07: 10: 47.52$ & 1.13 & 33.156 & -115.652 & 0.2 & 14891188 \\
\hline $2010 / 12 / 05$ & $09: 28: 40.76$ & 0.50 & 33.187 & -115.608 & 0.2 & 14892244 \\
\hline $2010 / 12 / 06$ & $02: 09: 14.00$ & 1.43 & 33.143 & -115.638 & 4.8 & 14892612 \\
\hline $2010 / 12 / 07$ & $09: 03: 13.49$ & 0.30 & 33.177 & -115.621 & 2.7 & 14893164 \\
\hline $2010 / 12 / 09$ & $09: 05: 55.12$ & 0.50 & 33.184 & -115.597 & 0.1 & 14894380 \\
\hline $2010 / 12 / 10$ & $03: 57: 56.86$ & 0.70 & 33.205 & -115.577 & 0.0 & 14894836 \\
\hline $2010 / 12 / 10$ & $04: 07: 42.01$ & 0.60 & 33.207 & -115.580 & 1.9 & 14894844 \\
\hline $2010 / 12 / 13$ & $20: 18: 28.75$ & 1.38 & 33.117 & -115.613 & 7.9 & 14897340 \\
\hline $2010 / 12 / 27$ & $02: 00: 14.58$ & 0.70 & 33.175 & -115.603 & 0.1 & 10862525 \\
\hline $2010 / 12 / 28$ & $02: 21: 55.47$ & 1.35 & 33.193 & -115.615 & 3.9 & 10863365 \\
\hline $2010 / 12 / 30$ & $08: 33: 37.27$ & 1.68 & 33.108 & -115.602 & 8.1 & 10864637 \\
\hline $2010 / 12 / 31$ & $10: 47: 33.54$ & 0.70 & 33.172 & -115.611 & 3.7 & 10864997 \\
\hline
\end{tabular}

Table 2. List of catalog master events used in MFP template creation 


\section{Appendix B}

List of New Events Identified In This Study

\begin{tabular}{|c|c|c|c|c|}
\hline Date & Time & Latitude & Longitude & Depth \\
\hline 2009/11/07 & $04: 36: 17.641$ & 33.196 & -115.559 & $0.2^{1}$ \\
\hline $2009 / 11 / 07$ & $04: 36: 57.641$ & 33.190 & -115.574 & 3.1 \\
\hline 2009/11/07 & $04: 48: 44.641$ & 33.170 & -115.600 & 1.1 \\
\hline $2009 / 11 / 07$ & $04: 51: 03.641$ & 33.188 & -115.573 & 0.2 \\
\hline $2009 / 11 / 07$ & $04: 53: 29.641$ & 33.196 & -115.559 & 0.2 \\
\hline $2009 / 11 / 07$ & $04: 55: 22.641$ & 33.189 & -115.576 & 0.1 \\
\hline $2009 / 11 / 07$ & 04:57:03.641 & 33.184 & -115.601 & 3.5 \\
\hline 2009/11/07 & 04:58:07.641 & 33.166 & -115.617 & 5.3 \\
\hline $2009 / 11 / 07$ & 05:04:06.641 & 33.190 & -115.574 & 3.1 \\
\hline $2009 / 11 / 07$ & 05:06:08.641 & 33.190 & -115.574 & 3.1 \\
\hline $2009 / 11 / 07$ & $05: 12: 48.641$ & 33.170 & -115.600 & 1.1 \\
\hline $2009 / 11 / 07$ & $05: 18: 34.641$ & 33.190 & -115.574 & 3.1 \\
\hline $2009 / 11 / 07$ & 05:19:09.641 & 33.194 & -115.583 & 3.0 \\
\hline $2009 / 11 / 07$ & $05: 19: 51.641$ & 33.194 & -115.583 & 3.0 \\
\hline $2009 / 11 / 07$ & $05: 21: 07.641$ & 33.207 & -115.567 & 3.7 \\
\hline $2009 / 11 / 07$ & $05: 22: 11.641$ & 33.190 & -115.574 & 3.1 \\
\hline $2009 / 11 / 07$ & $05: 25: 15.641$ & 33.193 & -115.615 & 3.9 \\
\hline $2009 / 11 / 07$ & $05: 26: 34.641$ & 33.196 & -115.559 & 0.2 \\
\hline $2009 / 11 / 07$ & $05: 34: 21.641$ & 33.181 & -115.608 & 1.0 \\
\hline $2009 / 11 / 07$ & $05: 44: 37.641$ & 33.194 & -115.583 & 3.0 \\
\hline $2009 / 11 / 07$ & $06: 11: 18.641$ & 33.208 & -115.582 & 2.5 \\
\hline $2009 / 11 / 07$ & $06: 13: 40.641$ & 33.189 & -115.576 & 0.1 \\
\hline $2009 / 11 / 07$ & $06: 13: 57.641$ & 33.193 & -115.583 & 5.9 \\
\hline $2009 / 11 / 07$ & $06: 14: 50.641$ & 33.179 & -115.594 & 1.5 \\
\hline $2009 / 11 / 07$ & $06: 15: 27.641$ & 33.203 & -115.578 & 2.2 \\
\hline 2009/11/07 & $06: 16: 41.641$ & 33.203 & -115.578 & 2.2 \\
\hline $2009 / 11 / 07$ & $06: 17: 10.641$ & 33.176 & -115.608 & 0.4 \\
\hline $2009 / 11 / 07$ & $06: 17: 36.641$ & 33.220 & -115.571 & 5.3 \\
\hline $2009 / 11 / 07$ & $06: 18: 13.641$ & 33.179 & -115.594 & 1.5 \\
\hline 2009/11/07 & $06: 20: 15.641$ & 33.203 & -115.578 & 2.2 \\
\hline 2009/11/07 & $06: 20: 37.641$ & 33.193 & -115.583 & 5.9 \\
\hline 2009/11/07 & $06: 23: 37.641$ & 33.176 & -115.608 & 0.4 \\
\hline $2009 / 11 / 07$ & $06: 25: 25.641$ & 33.203 & -115.578 & 2.2 \\
\hline 2009/11/07 & $06: 26: 03.641$ & 33.203 & -115.578 & 2.2 \\
\hline $2009 / 11 / 07$ & $06: 27: 23.641$ & 33.203 & -115.578 & 2.2 \\
\hline 2009/11/07 & $06: 28: 31.641$ & 33.203 & -115.578 & 2.2 \\
\hline 2009/11/07 & $06: 39: 39.641$ & 33.231 & -115.655 & 4.8 \\
\hline 2009/11/07 & $06: 43: 08.641$ & 33.170 & -115.600 & 1.1 \\
\hline
\end{tabular}




$\begin{array}{lllll}2009 / 11 / 07 & 06: 43: 56.641 & 33.176 & -115.608 & 0.4 \\ 2009 / 11 / 07 & 06: 45: 17.641 & 33.203 & -115.578 & 2.2 \\ 2009 / 11 / 07 & 06: 45: 37.641 & 33.193 & -115.583 & 5.9 \\ 2009 / 11 / 07 & 06: 46: 41.641 & 33.189 & -115.576 & 0.1 \\ 2009 / 11 / 07 & 06: 47: 33.641 & 33.188 & -115.573 & 0.2 \\ 2009 / 11 / 07 & 06: 47: 51.641 & 33.203 & -115.578 & 2.2 \\ 2009 / 11 / 07 & 06: 48: 23.641 & 33.203 & -115.578 & 2.2 \\ 2009 / 11 / 07 & 06: 49: 17.641 & 33.203 & -115.578 & 2.2 \\ 2009 / 11 / 07 & 06: 49: 58.641 & 33.194 & -115.583 & 3.0 \\ 2009 / 11 / 07 & 06: 50: 21.641 & 33.203 & -115.578 & 2.2 \\ 2009 / 11 / 07 & 06: 51: 23.641 & 33.203 & -115.578 & 2.2 \\ 2009 / 11 / 07 & 06: 51: 36.641 & 33.203 & -115.578 & 2.2 \\ 2009 / 11 / 07 & 06: 51: 51.641 & 33.203 & -115.578 & 2.2 \\ 2009 / 11 / 07 & 06: 52: 30.641 & 33.201 & -115.544 & 3.4 \\ 2009 / 11 / 07 & 06: 53: 02.641 & 33.198 & -115.571 & 0.2 \\ 2009 / 11 / 07 & 06: 53: 32.641 & 33.179 & -115.594 & 1.5 \\ 2009 / 11 / 07 & 06: 55: 04.641 & 33.198 & -115.571 & 0.2 \\ 2009 / 11 / 07 & 06: 59: 41.641 & 33.176 & -115.604 & 0.5 \\ 2009 / 11 / 07 & 07: 02: 05.641 & 33.194 & -115.583 & 3.0 \\ 2009 / 11 / 07 & 07: 02: 52.641 & 33.203 & -115.578 & 2.2 \\ 2009 / 11 / 07 & 07: 04: 20.641 & 33.226 & -115.588 & 4.6 \\ 2009 / 11 / 07 & 07: 04: 39.641 & 33.194 & -115.583 & 3.0 \\ 2009 / 11 / 07 & 07: 08: 31.641 & 33.196 & -115.587 & 5.3 \\ 2009 / 11 / 07 & 07: 13: 06.641 & 33.190 & -115.574 & 3.1 \\ 2009 / 11 / 07 & 07: 20: 33.641 & 33.194 & -115.583 & 3.0 \\ 2009 / 11 / 07 & 07: 21: 17.641 & 33.194 & -115.583 & 3.0 \\ 2009 / 11 / 07 & 07: 21: 57.641 & 33.194 & -115.583 & 3.0 \\ 2009 / 11 / 07 & 07: 22: 44.641 & 33.194 & -115.583 & 3.0 \\ 2009 / 11 / 07 & 07: 24: 51.641 & 33.194 & -115.583 & 3.0 \\ 2009 / 11 / 07 & 07: 28: 51.641 & 33.173 & -115.610 & 1.3 \\ 2009 / 11 / 07 & 07: 39: 39.641 & 33.176 & -115.606 & 6.3 \\ 2009 / 11 / 07 & 08: 02: 30.641 & 33.176 & -115.608 & 0.4 \\ 2009 / 11 / 07 & 08: 02: 53.641 & 33.176 & -115.608 & 0.4 \\ 2009 / 11 / 07 & 08: 03: 19.641 & 33.203 & -115.578 & 2.2 \\ 2009 / 11 / 07 & 08: 03: 48.641 & 33.203 & -115.578 & 2.2 \\ 2009 / 11 / 07 & 08: 04: 42.641 & 33.196 & -115.559 & 0.2 \\ 2009 / 11 / 07 & 08: 05: 11.641 & 33.203 & -115.578 & 2.2 \\ 2009 / 11 / 07 & 08: 05: 55.641 & 33.189 & -115.576 & 0.1 \\ 2009 / 11 / 07 & 08: 07: 46.641 & 33.193 & -115.583 & 5.9 \\ 2009 / 11 / 07 & 08: 08: 25.641 & 33.203 & -115.578 & 2.2 \\ 2009 / 11 / 07 & 08: 12: 41.641 & 33.203 & -115.578 & 2.2 \\ 2009 / 11 / 07 & 08: 28: 51.641 & 33.203 & -115.578 & 2.2 \\ 207 & 08: 32: 41.641 & 33.203 & -115.587 & 5.3 \\ 207 & 08: 53: 13.641 & 33.193 & -115.583 & 5.9\end{array}$




\begin{tabular}{lllll}
$2009 / 11 / 07$ & $08: 53: 32.641$ & 33.170 & -115.600 & 1.1 \\
$2009 / 11 / 07$ & $09: 12: 40.641$ & 33.179 & -115.594 & 1.5 \\
$2009 / 11 / 07$ & $09: 13: 37.641$ & 33.208 & -115.582 & 2.5 \\
$2009 / 11 / 07$ & $09: 55: 19.641$ & 33.203 & -115.578 & 2.2 \\
$2009 / 11 / 07$ & $10: 14: 24.641$ & 33.177 & -115.621 & 2.7 \\
$2009 / 11 / 07$ & $10: 16: 52.641$ & 33.181 & -115.665 & 4.2 \\
$2009 / 11 / 07$ & $10: 17: 49.641$ & 33.181 & -115.665 & 4.2 \\
$2009 / 11 / 07$ & $12: 06: 43.641$ & 33.170 & -115.600 & 1.1 \\
$2009 / 11 / 09$ & $04: 17: 22.641$ & 33.186 & -115.604 & 2.0 \\
$2009 / 11 / 09$ & $11: 03: 26.641$ & 33.179 & -115.597 & 0.1 \\
$2009 / 11 / 09$ & $11: 07: 51.641$ & 33.188 & -115.600 & 0.1 \\
$2009 / 11 / 09$ & $12: 22: 22.641$ & 33.153 & -115.638 & 10.1 \\
$2009 / 11 / 10$ & $03: 04: 15.641$ & 33.176 & -115.609 & 2.1 \\
$2009 / 11 / 10$ & $03: 18: 17.641$ & 33.179 & -115.597 & 0.1 \\
$2009 / 11 / 10$ & $07: 32: 17.641$ & 33.202 & -115.575 & 3.8 \\
$2009 / 11 / 10$ & $07: 32: 44.641$ & 33.173 & -115.609 & 0.1 \\
$2009 / 11 / 10$ & $07: 33: 32.641$ & 33.186 & -115.624 & 2.5 \\
$2009 / 11 / 10$ & $07: 33: 58.641$ & 33.202 & -115.575 & 3.8 \\
$2009 / 11 / 10$ & $07: 37: 32.641$ & 33.183 & -115.607 & 0.0 \\
$2009 / 11 / 10$ & $07: 38: 45.641$ & 33.202 & -115.671 & 6.3 \\
$2009 / 11 / 10$ & $07: 42: 54.641$ & 33.183 & -115.599 & 0.1 \\
$2009 / 11 / 10$ & $08: 59: 23.641$ & 33.183 & -115.599 & 0.1 \\
$2009 / 11 / 10$ & $09: 49: 30.641$ & 33.179 & -115.594 & 1.5 \\
$2009 / 11 / 10$ & $09: 52: 12.641$ & 33.183 & -115.599 & 0.1 \\
$2009 / 11 / 10$ & $09: 55: 13.641$ & 33.179 & -115.594 & 1.5 \\
$2009 / 11 / 10$ & $12: 22: 02.641$ & 33.181 & -115.611 & 0.4 \\
$2009 / 11 / 10$ & $12: 31: 32.641$ & 33.181 & -115.608 & 1.0 \\
$2009 / 11 / 10$ & $12: 34: 32.641$ & 33.181 & -115.608 & 1.0 \\
$2009 / 11 / 10$ & $12: 59: 11.641$ & 33.183 & -115.599 & 0.1 \\
$2009 / 11 / 10$ & $13: 03: 21.641$ & 33.183 & -115.599 & 0.1 \\
$2009 / 11 / 10$ & $13: 03: 50.641$ & 33.183 & -115.599 & 0.1 \\
$2009 / 11 / 10$ & $13: 05: 32.641$ & 33.183 & -115.599 & 0.1 \\
$2009 / 11 / 10$ & $13: 24: 53.641$ & 33.177 & -115.606 & 0.1 \\
$2009 / 11 / 10$ & $13: 26: 19.641$ & 33.183 & -115.607 & 0.0 \\
$2009 / 11 / 10$ & $13: 28: 14.641$ & 33.181 & -115.612 & 1.1 \\
$2009 / 11 / 10$ & $14: 59: 06.641$ & 33.170 & -115.600 & 1.1 \\
$2009 / 11 / 10$ & $18: 10: 36.641$ & 33.214 & -115.620 & 5.3 \\
$2009 / 11 / 10$ & $18: 29: 28.641$ & 33.181 & -115.610 & 0.9 \\
$2009 / 11 / 10$ & $22: 27: 26.641$ & 33.200 & -115.590 & 3.5 \\
$2009 / 11 / 10$ & $22: 34: 07.641$ & 33.200 & -115.590 & 3.5 \\
$2009 / 11 / 10$ & $22: 34: 50.641$ & 33.200 & -115.590 & 3.5 \\
$2009 / 11 / 10$ & $22: 40: 18.641$ & 33.200 & -115.590 & 3.5 \\
$2009 / 11 / 10$ & $22: 42: 27.641$ & 33.200 & -115.590 & 3.5 \\
$2009 / 11 / 12$ & $02: 01: 44.641$ & 33.178 & -115.632 & 2.3 \\
$2009 / 11 / 12$ & $02: 02: 34.641$ & 33.178 & -115.632 & 2.3 \\
\hline $2: 02: 57.641$ & 33.140 & -115.636 & 7.3
\end{tabular}




$\begin{array}{lllll}2009 / 11 / 12 & 02: 03: 18.641 & 33.176 & -115.609 & 2.1 \\ 2009 / 11 / 12 & 02: 03: 32.641 & 33.176 & -115.609 & 2.1 \\ 2009 / 11 / 12 & 02: 03: 51.641 & 33.177 & -115.606 & 0.1 \\ 2009 / 11 / 12 & 02: 05: 05.641 & 33.178 & -115.632 & 2.3 \\ 2009 / 11 / 12 & 02: 05: 40.641 & 33.178 & -115.632 & 2.3 \\ 2009 / 11 / 12 & 02: 06: 46.641 & 33.178 & -115.632 & 2.3 \\ 2009 / 11 / 12 & 02: 07: 18.641 & 33.136 & -115.649 & 5.3 \\ 2009 / 11 / 12 & 02: 10: 07.641 & 33.177 & -115.606 & 0.1 \\ 2009 / 11 / 12 & 02: 19: 55.641 & 33.178 & -115.632 & 2.3 \\ 2009 / 11 / 12 & 02: 20: 31.641 & 33.176 & -115.609 & 2.1 \\ 2009 / 11 / 12 & 02: 21: 18.641 & 33.177 & -115.606 & 0.1 \\ 2009 / 11 / 12 & 02: 32: 17.641 & 33.176 & -115.609 & 2.1 \\ 2009 / 11 / 12 & 02: 40: 25.641 & 33.177 & -115.606 & 0.1 \\ 2009 / 11 / 12 & 03: 44: 04.641 & 33.158 & -115.638 & 0.4 \\ 2009 / 11 / 12 & 06: 59: 37.641 & 33.231 & -115.605 & 4.8 \\ 2009 / 11 / 12 & 07: 23: 27.641 & 33.118 & -115.590 & 13.8 \\ 2009 / 11 / 12 & 10: 42: 11.641 & 33.183 & -115.599 & 0.1 \\ 2009 / 11 / 12 & 13: 55: 55.641 & 33.108 & -115.602 & 8.1 \\ 2009 / 11 / 12 & 23: 20: 20.641 & 33.143 & -115.638 & 4.8 \\ 2009 / 11 / 12 & 23: 57: 15.641 & 33.100 & -115.590 & 14.6 \\ 2009 / 11 / 13 & 01: 55: 27.641 & 33.176 & -115.609 & 2.1 \\ 2009 / 11 / 13 & 02: 33: 07.641 & 33.108 & -115.602 & 8.1 \\ 2009 / 11 / 13 & 09: 08: 34.641 & 33.226 & -115.588 & 4.6 \\ 2009 / 11 / 13 & 21: 01: 35.641 & 33.231 & -115.655 & 4.8 \\ 2009 / 11 / 14 & 05: 24: 55.641 & 33.117 & -115.613 & 7.9 \\ 2009 / 11 / 14 & 12: 45: 38.641 & 33.108 & -115.602 & 8.1 \\ 2009 / 11 / 15 & 04: 22: 56.641 & 33.183 & -115.617 & 4.3 \\ 2009 / 11 / 15 & 04: 22: 57.641 & 33.193 & -115.583 & 5.9 \\ 2009 / 11 / 15 & 17: 08: 38.641 & 33.158 & -115.642 & 0.1 \\ 2009 / 11 / 16 & 02: 15: 57.641 & 33.100 & -115.590 & 14.6 \\ 2009 / 11 / 16 & 11: 29: 37.641 & 33.158 & -115.642 & 0.1 \\ 2009 / 11 / 16 & 23: 11: 14.641 & 33.229 & -115.645 & 5.7 \\ 2009 / 11 / 17 & 01: 41: 36.641 & 33.176 & -115.604 & 0.5 \\ 2009 / 11 / 17 & 13: 34: 28.641 & 33.158 & -115.642 & 0.1 \\ 2009 / 11 / 17 & 13: 49: 12.641 & 33.181 & -115.665 & 4.2 \\ 2009 / 11 / 17 & 14: 11: 42.641 & 33.117 & -115.613 & 7.9 \\ 2009 / 11 / 17 & 14: 33: 46.641 & 33.176 & -115.604 & 0.5 \\ 2009 / 11 / 17 & 14: 34: 50.641 & 33.100 & -115.590 & 14.6 \\ 2009 / 11 / 17 & 14: 49: 36.641 & 33.170 & -115.600 & 1.1 \\ 2009 / 11 / 17 & 15: 50: 31.641 & 33.192 & -115.567 & 3.1 \\ 2009 / 11 / 17 & 19: 27: 06.641 & 33.188 & -115.573 & 0.2 \\ 2009 / 11 / 17 & 19: 29: 45.641 & 33.188 & -115.573 & 0.2 \\ 2009 / 11 / 17 & 19: 52: 51.641 & 33.176 & -115.608 & 0.4 \\ 2009 / 11 / 17 & 20: 23: 34.641 & 33.176 & -115.604 & 0.5 \\ 2009 / 11 / 17 & 20: 30: 46.641 & 33.170 & -115.600 & 1.1 \\ 2009 / 11 / 17 & 22: 25: 57.641 & 33.176 & -115.608 & 0.4\end{array}$




$\begin{array}{lllll}2009 / 11 / 17 & 22: 26: 21.641 & 33.176 & -115.608 & 0.4 \\ 2009 / 11 / 17 & 22: 37: 43.641 & 33.176 & -115.609 & 2.1 \\ 2009 / 11 / 17 & 22: 39: 55.641 & 33.176 & -115.604 & 0.5 \\ 2009 / 11 / 17 & 22: 43: 08.641 & 33.179 & -115.594 & 1.5 \\ 2009 / 11 / 17 & 22: 52: 10.641 & 33.184 & -115.597 & 0.1 \\ 2009 / 11 / 17 & 22: 53: 05.641 & 33.176 & -115.609 & 2.1 \\ 2009 / 11 / 17 & 22: 54: 26.641 & 33.176 & -115.609 & 2.1 \\ 2009 / 11 / 17 & 22: 54: 58.641 & 33.176 & -115.604 & 0.5 \\ 2009 / 11 / 17 & 22: 55: 31.641 & 33.196 & -115.587 & 5.3 \\ 2009 / 11 / 17 & 22: 56: 07.641 & 33.176 & -115.609 & 2.1 \\ 2009 / 11 / 17 & 22: 57: 01.641 & 33.176 & -115.609 & 2.1 \\ 2009 / 11 / 17 & 22: 58: 16.641 & 33.177 & -115.606 & 0.1 \\ 2009 / 11 / 17 & 22: 58: 31.641 & 33.193 & -115.583 & 5.9 \\ 2009 / 11 / 17 & 23: 01: 25.641 & 33.176 & -115.609 & 2.1 \\ 2009 / 11 / 17 & 23: 01: 38.641 & 33.176 & -115.609 & 2.1 \\ 2009 / 11 / 17 & 23: 01: 55.641 & 33.176 & -115.609 & 2.1 \\ 2009 / 11 / 17 & 23: 02: 30.641 & 33.176 & -115.609 & 2.1 \\ 2009 / 11 / 17 & 23: 04: 17.641 & 33.176 & -115.609 & 2.1 \\ 2009 / 11 / 17 & 23: 05: 34.641 & 33.177 & -115.614 & 1.2 \\ 2009 / 11 / 17 & 23: 06: 39.641 & 33.182 & -115.602 & 2.5 \\ 2009 / 11 / 17 & 23: 07: 13.641 & 33.176 & -115.608 & 0.4 \\ 2009 / 11 / 17 & 23: 07: 55.641 & 33.176 & -115.608 & 0.4 \\ 2009 / 11 / 17 & 23: 08: 11.641 & 33.196 & -115.587 & 5.3 \\ 2009 / 11 / 17 & 23: 08: 48.641 & 33.197 & -115.614 & 5.3 \\ 2009 / 11 / 17 & 23: 09: 07.641 & 33.183 & -115.599 & 0.1 \\ 2009 / 11 / 17 & 23: 09: 50.641 & 33.214 & -115.620 & 5.3 \\ 2009 / 11 / 17 & 23: 10: 07.641 & 33.182 & -115.602 & 2.5 \\ 2009 / 11 / 17 & 23: 10: 40.641 & 33.181 & -115.665 & 4.2 \\ 2009 / 11 / 17 & 23: 11: 37.641 & 33.176 & -115.609 & 2.1 \\ 2009 / 11 / 17 & 23: 12: 31.641 & 33.176 & -115.608 & 0.4 \\ 2009 / 11 / 17 & 23: 13: 21.641 & 33.193 & -115.602 & 0.0 \\ 2009 / 11 / 17 & 23: 13: 55.641 & 33.188 & -115.599 & 0.8 \\ 2009 / 11 / 17 & 23: 15: 07.641 & 33.229 & -115.645 & 5.7 \\ 2009 / 11 / 17 & 23: 15: 31.641 & 33.197 & -115.614 & 5.3 \\ 2009 / 11 / 17 & 23: 16: 20.641 & 33.181 & -115.608 & 1.0 \\ 2009 / 11 / 17 & 23: 16: 43.641 & 33.176 & -115.609 & 2.1 \\ 2009 / 11 / 17 & 23: 17: 14.641 & 33.229 & -115.645 & 5.7 \\ 2009 / 11 / 17 & 23: 17: 53.641 & 33.229 & -115.645 & 5.7 \\ 2009 / 11 / 17 & 23: 18: 21.641 & 33.176 & -115.609 & 2.1 \\ 2009 / 11 / 17 & 23: 18: 33.641 & 33.179 & -115.594 & 1.5 \\ 2009 / 11 / 17 & 23: 19: 14.641 & 33.229 & -115.645 & 5.7 \\ 2009 / 11 / 17 & 23: 20: 12.641 & 33.172 & -115.610 & 2.2 \\ 2009 / 11 / 17 & 23: 20: 30.641 & 33.196 & -115.587 & 5.3 \\ 2009 / 11 / 17 & 23: 21: 28.641 & 33.176 & -115.609 & 2.1 \\ 23: 24: 05.641 & 33.181 & -115.665 & 4.2 \\ 23: 24: 39.641 & 33.176 & -115.609 & 2.1\end{array}$




$\begin{array}{lllll}2009 / 11 / 17 & 23: 25: 40.641 & 33.176 & -115.604 & 0.5 \\ 2009 / 11 / 17 & 23: 26: 14.641 & 33.197 & -115.614 & 5.3 \\ 2009 / 11 / 17 & 23: 27: 32.641 & 33.197 & -115.614 & 5.3 \\ 2009 / 11 / 17 & 23: 28: 11.641 & 33.172 & -115.610 & 2.2 \\ 2009 / 11 / 17 & 23: 28: 33.641 & 33.176 & -115.609 & 2.1 \\ 2009 / 11 / 17 & 23: 30: 57.641 & 33.197 & -115.614 & 5.3 \\ 2009 / 11 / 17 & 23: 35: 26.641 & 33.176 & -115.609 & 2.1 \\ 2009 / 11 / 17 & 23: 41: 40.641 & 33.179 & -115.594 & 1.5 \\ 2009 / 11 / 17 & 23: 42: 27.641 & 33.181 & -115.608 & 1.0 \\ 2009 / 11 / 17 & 23: 43: 21.641 & 33.179 & -115.594 & 1.5 \\ 2009 / 11 / 17 & 23: 45: 02.641 & 33.176 & -115.608 & 0.4 \\ 2009 / 11 / 17 & 23: 45: 31.641 & 33.176 & -115.608 & 0.4 \\ 2009 / 11 / 17 & 23: 47: 00.641 & 33.177 & -115.621 & 2.7 \\ 2009 / 11 / 17 & 23: 48: 51.641 & 33.179 & -115.594 & 1.5 \\ 2009 / 11 / 17 & 23: 50: 50.641 & 33.180 & -115.600 & 0.1 \\ 2009 / 11 / 17 & 23: 55: 15.641 & 33.193 & -115.615 & 3.9 \\ 2009 / 11 / 17 & 23: 55: 55.641 & 33.179 & -115.594 & 1.5 \\ 2009 / 11 / 17 & 23: 56: 21.641 & 33.179 & -115.594 & 1.5 \\ 2009 / 11 / 17 & 23: 58: 01.641 & 33.179 & -115.594 & 1.5 \\ 2009 / 11 / 18 & 00: 00: 55.641 & 33.192 & -115.579 & 4.3 \\ 2009 / 11 / 18 & 00: 04: 05.641 & 33.207 & -115.567 & 3.7 \\ 2009 / 11 / 18 & 00: 04: 06.641 & 33.193 & -115.583 & 5.9 \\ 2009 / 11 / 18 & 00: 05: 04.641 & 33.231 & -115.605 & 4.8 \\ 2009 / 11 / 18 & 00: 11: 43.641 & 33.182 & -115.606 & 1.7 \\ 2009 / 11 / 18 & 00: 12: 16.641 & 33.173 & -115.609 & 0.1 \\ 2009 / 11 / 18 & 00: 12: 51.641 & 33.173 & -115.609 & 0.1 \\ 2009 / 11 / 18 & 00: 16: 53.641 & 33.214 & -115.620 & 5.3 \\ 2009 / 11 / 18 & 00: 20: 02.641 & 33.181 & -115.608 & 1.0 \\ 2009 / 11 / 18 & 00: 20: 54.641 & 33.190 & -115.604 & 1.2 \\ 2009 / 11 / 18 & 00: 22: 40.641 & 33.182 & -115.602 & 2.5 \\ 2009 / 11 / 18 & 00: 23: 05.641 & 33.197 & -115.614 & 5.3 \\ 2009 / 11 / 18 & 00: 26: 11.641 & 33.173 & -115.609 & 0.1 \\ 2009 / 11 / 18 & 00: 28: 08.641 & 33.176 & -115.609 & 2.1 \\ 2009 / 11 / 18 & 00: 28: 41.641 & 33.176 & -115.609 & 2.1 \\ 2009 / 11 / 18 & 00: 34: 49.641 & 33.108 & -115.602 & 8.1 \\ 2009 / 11 / 18 & 00: 35: 20.641 & 33.231 & -115.605 & 4.8 \\ 2009 / 11 / 18 & 00: 43: 01.641 & 33.179 & -115.594 & 1.5 \\ 2009 / 11 / 18 & 00: 43: 58.641 & 33.182 & -115.602 & 2.5 \\ 2009 / 11 / 18 & 00: 44: 59.641 & 33.176 & -115.609 & 2.1 \\ 2009 / 11 / 18 & 00: 45: 52.641 & 33.181 & -115.665 & 4.2 \\ 2009 / 11 / 18 & 00: 46: 30.641 & 33.176 & -115.609 & 2.1 \\ 2009 / 11 / 18 & 00: 47: 10.641 & 33.176 & -115.609 & 2.1 \\ 2009 / 11 / 18 & 00: 48: 36.641 & 33.176 & -115.587 & 5.3 \\ 200: 49: 10.641 & 33.188 & -115.609 & 2.1 \\ 200: 49: 31.641 & 33.181 & -115.665 & 4.2\end{array}$




$\begin{array}{lllll}2009 / 11 / 18 & 00: 49: 59.641 & 33.176 & -115.604 & 0.5 \\ 2009 / 11 / 18 & 00: 50: 54.641 & 33.179 & -115.594 & 1.5 \\ 2009 / 11 / 18 & 00: 51: 18.641 & 33.181 & -115.665 & 4.2 \\ 2009 / 11 / 18 & 00: 52: 39.641 & 33.188 & -115.573 & 0.2 \\ 2009 / 11 / 18 & 00: 52: 51.641 & 33.181 & -115.665 & 4.2 \\ 2009 / 11 / 18 & 00: 54: 05.641 & 33.229 & -115.645 & 5.7 \\ 2009 / 11 / 18 & 00: 55: 01.641 & 33.190 & -115.604 & 1.2 \\ 2009 / 11 / 18 & 00: 55: 48.641 & 33.176 & -115.609 & 2.1 \\ 2009 / 11 / 18 & 00: 56: 31.641 & 33.181 & -115.665 & 4.2 \\ 2009 / 11 / 18 & 00: 56: 47.641 & 33.176 & -115.609 & 2.1 \\ 2009 / 11 / 18 & 00: 57: 16.641 & 33.181 & -115.665 & 4.2 \\ 2009 / 11 / 18 & 00: 57: 43.641 & 33.176 & -115.604 & 0.5 \\ 2009 / 11 / 18 & 00: 59: 29.641 & 33.177 & -115.621 & 2.7 \\ 2009 / 11 / 18 & 00: 59: 45.641 & 33.176 & -115.608 & 0.4 \\ 2009 / 11 / 18 & 01: 01: 07.641 & 33.181 & -115.665 & 4.2 \\ 2009 / 11 / 18 & 01: 01: 39.641 & 33.181 & -115.665 & 4.2 \\ 2009 / 11 / 18 & 01: 02: 19.641 & 33.181 & -115.665 & 4.2 \\ 2009 / 11 / 18 & 01: 04: 29.641 & 33.186 & -115.614 & 2.4 \\ 2009 / 11 / 18 & 01: 05: 14.641 & 33.171 & -115.605 & 0.7 \\ 2009 / 11 / 18 & 01: 06: 41.641 & 33.181 & -115.665 & 4.2 \\ 2009 / 11 / 18 & 01: 07: 12.641 & 33.176 & -115.604 & 0.5 \\ 2009 / 11 / 18 & 01: 07: 33.641 & 33.229 & -115.645 & 5.7 \\ 2009 / 11 / 18 & 01: 08: 22.641 & 33.176 & -115.609 & 2.1 \\ 2009 / 11 / 18 & 01: 09: 37.641 & 33.176 & -115.609 & 2.1 \\ 2009 / 11 / 18 & 01: 09: 59.641 & 33.229 & -115.645 & 5.7 \\ 2009 / 11 / 18 & 01: 11: 04.641 & 33.176 & -115.609 & 2.1 \\ 2009 / 11 / 18 & 01: 12: 21.641 & 33.214 & -115.620 & 5.3 \\ 2009 / 11 / 18 & 01: 13: 19.641 & 33.186 & -115.604 & 2.0 \\ 2009 / 11 / 18 & 01: 14: 46.641 & 33.229 & -115.645 & 5.7 \\ 2009 / 11 / 18 & 01: 15: 26.641 & 33.171 & -115.605 & 0.7 \\ 2009 / 11 / 18 & 01: 16: 02.641 & 33.182 & -115.603 & 0.1 \\ 2009 / 11 / 18 & 01: 21: 57.641 & 33.181 & -115.665 & 4.2 \\ 2009 / 11 / 18 & 01: 22: 19.641 & 33.176 & -115.609 & 2.1 \\ 2009 / 11 / 18 & 01: 22: 57.641 & 33.181 & -115.665 & 4.2 \\ 2009 / 11 / 18 & 01: 24: 06.641 & 33.179 & -115.594 & 1.5 \\ 2009 / 11 / 18 & 01: 25: 23.641 & 33.229 & -115.645 & 5.7 \\ 2009 / 11 / 18 & 01: 29: 25.641 & 33.181 & -115.608 & 1.0 \\ 2009 / 11 / 18 & 01: 30: 07.641 & 33.181 & -115.665 & 4.2 \\ 2009 / 11 / 18 & 01: 31: 03.641 & 33.181 & -115.665 & 4.2 \\ 2009 / 11 / 18 & 01: 34: 05.641 & 33.197 & -115.614 & 5.3 \\ 2009 / 11 / 18 & 01: 34: 32.641 & 33.197 & -115.614 & 5.3 \\ 2009 / 11 / 18 & 01: 35: 14.641 & 33.181 & -115.665 & 4.2 \\ 2009 / 11 / 18 & 01: 36: 17.641 & 33.180 & -115.594 & 1.5 \\ 20 & 01: 37: 43.641 & -115.600 & 0.1 \\ 23.641 & 33.181 & -115.665 & 4.2\end{array}$




\begin{tabular}{lllll}
$2009 / 11 / 18$ & $01: 41: 48.641$ & 33.184 & -115.605 & 0.1 \\
$2009 / 11 / 18$ & $01: 43: 11.641$ & 33.231 & -115.605 & 4.8 \\
$2009 / 11 / 18$ & $01: 44: 12.641$ & 33.176 & -115.609 & 2.1 \\
$2009 / 11 / 18$ & $01: 45: 00.641$ & 33.176 & -115.609 & 2.1 \\
$2009 / 11 / 18$ & $01: 47: 17.641$ & 33.193 & -115.615 & 3.9 \\
$2009 / 11 / 18$ & $01: 50: 42.641$ & 33.188 & -115.599 & 0.8 \\
$2009 / 11 / 18$ & $01: 51: 14.641$ & 33.181 & -115.608 & 0.0 \\
$2009 / 11 / 18$ & $01: 51: 50.641$ & 33.186 & -115.612 & 0.6 \\
$2009 / 11 / 18$ & $01: 53: 04.641$ & 33.231 & -115.605 & 4.8 \\
$2009 / 11 / 18$ & $01: 54: 29.641$ & 33.186 & -115.612 & 0.6 \\
$2009 / 11 / 18$ & $01: 54: 50.641$ & 33.181 & -115.665 & 4.2 \\
$2009 / 11 / 18$ & $01: 55: 54.641$ & 33.182 & -115.603 & 0.1 \\
$2009 / 11 / 18$ & $01: 56: 35.641$ & 33.181 & -115.601 & 0.7 \\
$2009 / 11 / 18$ & $01: 57: 46.641$ & 33.182 & -115.603 & 0.1 \\
$2009 / 11 / 18$ & $01: 58: 43.641$ & 33.182 & -115.603 & 0.1 \\
$2009 / 11 / 18$ & $01: 59: 33.641$ & 33.214 & -115.620 & 5.3 \\
$2009 / 11 / 18$ & $02: 02: 41.641$ & 33.197 & -115.614 & 5.3 \\
$2009 / 11 / 18$ & $02: 06: 47.641$ & 33.229 & -115.645 & 5.7 \\
$2009 / 11 / 18$ & $02: 07: 44.641$ & 33.181 & -115.665 & 4.2 \\
$2009 / 11 / 18$ & $02: 08: 23.641$ & 33.181 & -115.665 & 4.2 \\
$2009 / 11 / 18$ & $02: 08: 55.641$ & 33.179 & -115.594 & 1.5 \\
$2009 / 11 / 18$ & $02: 09: 21.641$ & 33.181 & -115.665 & 4.2 \\
$2009 / 11 / 18$ & $02: 10: 08.641$ & 33.181 & -115.603 & 1.4 \\
$2009 / 11 / 18$ & $02: 11: 09.641$ & 33.184 & -115.605 & 0.1 \\
$2009 / 11 / 18$ & $02: 12: 54.641$ & 33.172 & -115.610 & 2.2 \\
$2009 / 11 / 18$ & $02: 13: 23.641$ & 33.173 & -115.609 & 0.1 \\
$2009 / 11 / 18$ & $02: 14: 32.641$ & 33.182 & -115.606 & 1.7 \\
$2009 / 11 / 18$ & $02: 16: 01.641$ & 33.184 & -115.605 & 0.1 \\
$2009 / 11 / 18$ & $02: 22: 10.641$ & 33.184 & -115.605 & 0.1 \\
$2009 / 11 / 18$ & $02: 26: 14.641$ & 33.179 & -115.594 & 1.5 \\
$2009 / 11 / 18$ & $02: 27: 02.641$ & 33.188 & -115.609 & 2.3 \\
$2009 / 11 / 18$ & $02: 29: 51.641$ & 33.231 & -115.605 & 4.8 \\
$2009 / 11 / 18$ & $02: 30: 31.641$ & 33.176 & -115.609 & 2.1 \\
$2009 / 11 / 18$ & $02: 35: 06.641$ & 33.181 & -115.608 & 0.0 \\
$2009 / 11 / 18$ & $02: 36: 41.641$ & 33.186 & -115.612 & 0.6 \\
$2009 / 11 / 18$ & $02: 37: 46.641$ & 33.184 & -115.601 & 3.5 \\
$2009 / 11 / 18$ & $02: 38: 19.641$ & 33.182 & -115.603 & 0.1 \\
$2009 / 11 / 18$ & $02: 39: 21.641$ & 33.182 & -115.603 & 0.1 \\
$2009 / 11 / 18$ & $02: 43: 34.641$ & 33.186 & -115.612 & 0.6 \\
$2009 / 11 / 18$ & $02: 43: 56.641$ & 33.181 & -115.611 & 0.4 \\
$2009 / 11 / 18$ & $02: 44: 10.641$ & 33.197 & -115.614 & 5.3 \\
$2009 / 11 / 11 / 18$ & $02: 45: 37.641$ & 33.182 & -115.603 & 0.1 \\
\hline & $02: 52: 09.641$ & 33.186 & -115.604 & 2.0 \\
$202: 53: 53.641$ & 33.186 & -115.612 & 0.6 \\
20 & $02: 56: 43.641$ & 33.197 & -115.614 & 5.3 \\
$201: 25.641$ & 33.173 & -115.609 & 0.1
\end{tabular}




$\begin{array}{lllll}2009 / 11 / 18 & 03: 02: 30.641 & 33.173 & -115.609 & 0.1 \\ 2009 / 11 / 18 & 03: 05: 32.641 & 33.197 & -115.614 & 5.3 \\ 2009 / 11 / 18 & 03: 07: 00.641 & 33.176 & -115.609 & 2.1 \\ 2009 / 11 / 18 & 03: 10: 58.641 & 33.182 & -115.606 & 1.7 \\ 2009 / 11 / 18 & 03: 17: 54.641 & 33.182 & -115.603 & 0.1 \\ 2009 / 11 / 18 & 03: 21: 50.641 & 33.229 & -115.645 & 5.7 \\ 2009 / 11 / 18 & 03: 23: 06.641 & 33.196 & -115.587 & 5.3 \\ 2009 / 11 / 18 & 03: 29: 52.641 & 33.170 & -115.600 & 1.1 \\ 2009 / 11 / 18 & 03: 31: 28.641 & 33.176 & -115.604 & 0.5 \\ 2009 / 11 / 18 & 03: 34: 21.641 & 33.192 & -115.626 & 7.7 \\ 2009 / 11 / 18 & 03: 37: 34.641 & 33.231 & -115.605 & 4.8 \\ 2009 / 11 / 18 & 03: 38: 01.641 & 33.181 & -115.611 & 0.4 \\ 2009 / 11 / 18 & 03: 47: 42.641 & 33.184 & -115.605 & 0.1 \\ 2009 / 11 / 18 & 03: 51: 07.641 & 33.193 & -115.583 & 5.9 \\ 2009 / 11 / 18 & 03: 53: 41.641 & 33.197 & -115.614 & 5.3 \\ 2009 / 11 / 18 & 03: 59: 46.641 & 33.190 & -115.608 & 2.2 \\ 2009 / 11 / 18 & 04: 15: 33.641 & 33.172 & -115.609 & 0.1 \\ 2009 / 11 / 18 & 04: 25: 30.641 & 33.185 & -115.569 & 3.7 \\ 2009 / 11 / 18 & 04: 30: 47.641 & 33.184 & -115.605 & 0.1 \\ 2009 / 11 / 18 & 04: 37: 08.641 & 33.170 & -115.600 & 1.1 \\ 2009 / 11 / 18 & 04: 38: 17.641 & 33.176 & -115.609 & 2.1 \\ 2009 / 11 / 18 & 04: 38: 43.641 & 33.176 & -115.609 & 2.1 \\ 2009 / 11 / 18 & 04: 39: 06.641 & 33.176 & -115.609 & 2.1 \\ 2009 / 11 / 18 & 04: 46: 32.641 & 33.176 & -115.609 & 2.1 \\ 2009 / 11 / 18 & 04: 51: 58.641 & 33.231 & -115.605 & 4.8 \\ 2009 / 11 / 18 & 05: 09: 02.641 & 33.186 & -115.612 & 0.6 \\ 2009 / 11 / 18 & 05: 12: 46.641 & 33.188 & -115.609 & 2.3 \\ 2009 / 11 / 18 & 05: 25: 51.641 & 33.190 & -115.604 & 1.2 \\ 2009 / 11 / 18 & 05: 31: 12.641 & 33.226 & -115.588 & 4.6 \\ 2009 / 11 / 18 & 07: 14: 17.641 & 33.173 & -115.610 & 1.3 \\ 2009 / 11 / 18 & 07: 25: 34.641 & 33.172 & -115.610 & 2.2 \\ 2009 / 11 / 18 & 07: 28: 22.641 & 33.173 & -115.610 & 1.3 \\ 2009 / 11 / 18 & 07: 45: 27.641 & 33.172 & -115.609 & 0.1 \\ 2009 / 11 / 18 & 07: 50: 38.641 & 33.197 & -115.614 & 5.3 \\ 2009 / 11 / 18 & 08: 10: 36.641 & 33.171 & -115.605 & 0.7 \\ 2009 / 11 / 18 & 08: 14: 35.641 & 33.171 & -115.605 & 0.7 \\ 2009 / 11 / 18 & 08: 23: 29.641 & 33.197 & -115.614 & 5.3 \\ 2009 / 11 / 18 & 09: 01: 19.641 & 33.176 & -115.604 & 0.0 \\ 2009 / 11 / 18 & 09: 01: 54.641 & 33.181 & -115.659 & 6.0 \\ 2009 / 11 / 18 & 09: 13: 41.641 & 33.176 & -115.608 & 0.4 \\ 2009 / 11 / 18 & 09: 24: 18.641 & 33.197 & -115.614 & 5.3 \\ 2009 / 11 / 18 & 09: 25: 40.641 & 33.176 & -115.604 & 0.0 \\ 2009 / 11 / 18 & 10: 17: 44.641 & 33.173 & -115.609 & 0.1 \\ 2009 / 11 / 18 & 10: 19: 46.641 & 33.182 & -115.606 & 1.7\end{array}$




$\begin{array}{lllll}2009 / 11 / 18 & 10: 28: 23.641 & 33.172 & -115.609 & 0.1 \\ 2009 / 11 / 18 & 10: 30: 51.641 & 33.181 & -115.608 & 1.0 \\ 2009 / 11 / 18 & 10: 34: 25.641 & 33.172 & -115.609 & 0.1 \\ 2009 / 11 / 18 & 10: 35: 24.641 & 33.176 & -115.609 & 2.1 \\ 2009 / 11 / 18 & 10: 36: 09.641 & 33.196 & -115.587 & 5.3 \\ 2009 / 11 / 18 & 10: 36: 59.641 & 33.176 & -115.609 & 2.1 \\ 2009 / 11 / 18 & 10: 37: 35.641 & 33.176 & -115.609 & 2.1 \\ 2009 / 11 / 18 & 10: 38: 30.641 & 33.176 & -115.609 & 2.1 \\ 2009 / 11 / 18 & 10: 38: 55.641 & 33.176 & -115.609 & 2.1 \\ 2009 / 11 / 18 & 10: 42: 33.641 & 33.173 & -115.610 & 1.3 \\ 2009 / 11 / 18 & 10: 43: 57.641 & 33.196 & -115.587 & 5.3 \\ 2009 / 11 / 18 & 10: 44: 44.641 & 33.176 & -115.609 & 2.1 \\ 2009 / 11 / 18 & 10: 48: 35.641 & 33.196 & -115.587 & 5.3 \\ 2009 / 11 / 18 & 10: 56: 14.641 & 33.172 & -115.610 & 2.2 \\ 2009 / 11 / 18 & 11: 01: 51.641 & 33.176 & -115.608 & 0.4 \\ 2009 / 11 / 18 & 11: 02: 14.641 & 33.199 & -115.617 & 0.0 \\ 2009 / 11 / 18 & 11: 04: 11.641 & 33.181 & -115.608 & 0.0 \\ 2009 / 11 / 18 & 11: 04: 56.641 & 33.190 & -115.608 & 2.2 \\ 2009 / 11 / 18 & 11: 05: 19.641 & 33.181 & -115.608 & 0.0 \\ 2009 / 11 / 18 & 11: 05: 55.641 & 33.181 & -115.608 & 0.0 \\ 2009 / 11 / 18 & 11: 08: 11.641 & 33.192 & -115.626 & 7.7 \\ 2009 / 11 / 18 & 11: 08: 37.641 & 33.173 & -115.609 & 0.1 \\ 2009 / 11 / 18 & 11: 11: 01.641 & 33.186 & -115.604 & 2.0 \\ 2009 / 11 / 18 & 11: 11: 39.641 & 33.190 & -115.608 & 2.2 \\ 2009 / 11 / 18 & 11: 12: 21.641 & 33.231 & -115.605 & 4.8 \\ 2009 / 11 / 18 & 11: 14: 19.641 & 33.176 & -115.609 & 2.1 \\ 2009 / 11 / 18 & 11: 14: 54.641 & 33.181 & -115.665 & 4.2 \\ 2009 / 11 / 18 & 11: 23: 13.641 & 33.184 & -115.605 & 0.1 \\ 2009 / 11 / 18 & 11: 27: 24.641 & 33.184 & -115.605 & 0.1 \\ 2009 / 11 / 18 & 11: 33: 38.641 & 33.184 & -115.605 & 0.1 \\ 2009 / 11 / 18 & 11: 39: 15.641 & 33.170 & -115.600 & 1.1 \\ 2009 / 11 / 18 & 11: 57: 43.641 & 33.171 & -115.605 & 0.7 \\ 2009 / 11 / 18 & 12: 02: 50.641 & 33.118 & -115.610 & 9.1 \\ 2009 / 11 / 18 & 12: 31: 15.641 & 33.181 & -115.608 & 0.0 \\ 2009 / 11 / 18 & 12: 53: 57.641 & 33.186 & -115.614 & 2.4 \\ 2009 / 11 / 18 & 12: 55: 30.641 & 33.186 & -115.614 & 2.4 \\ 2009 / 11 / 18 & 13: 02: 01.641 & 33.186 & -115.614 & 2.4 \\ 2009 / 11 / 18 & 13: 13: 54.641 & 33.186 & -115.614 & 2.4 \\ 2009 / 11 / 18 & 13: 24: 38.641 & 33.182 & -115.602 & 2.5 \\ 2009 / 11 / 18 & 13: 37: 14.641 & 33.135 & -115.644 & 6.9 \\ 2009 / 11 / 18 & 13: 37: 36.641 & 33.178 & -115.611 & 1.6 \\ 2009 / 11 / 18 & 13: 39: 46.641 & 33.178 & -115.611 & 1.6 \\ 2009 / 11 / 18 & 13: 40: 37.641 & 33.178 & -115.611 & 1.6 \\ 209 / 11 / 18 & 13: 47: 09.641 & 33.171 & -115.605 & 0.7\end{array}$




\begin{tabular}{lllll}
$2009 / 11 / 18$ & $14: 42: 41.641$ & 33.172 & -115.610 & 2.2 \\
$2009 / 11 / 18$ & $15: 16: 34.641$ & 33.177 & -115.606 & 0.1 \\
$2009 / 11 / 18$ & $15: 23: 24.641$ & 33.108 & -115.602 & 8.1 \\
$2009 / 11 / 18$ & $17: 30: 50.641$ & 33.186 & -115.614 & 2.4 \\
$2009 / 11 / 18$ & $17: 47: 37.641$ & 33.172 & -115.610 & 2.2 \\
$2009 / 11 / 18$ & $18: 25: 42.641$ & 33.171 & -115.605 & 0.7 \\
$2009 / 11 / 18$ & $18: 29: 07.641$ & 33.171 & -115.605 & 0.7 \\
$2009 / 11 / 18$ & $18: 29: 37.641$ & 33.171 & -115.605 & 0.7 \\
$2009 / 11 / 18$ & $19: 59: 53.641$ & 33.177 & -115.621 & 2.7 \\
$2009 / 11 / 18$ & $20: 00: 49.641$ & 33.178 & -115.611 & 1.6 \\
$2009 / 11 / 18$ & $20: 20: 30.641$ & 33.202 & -115.671 & 6.3 \\
$2009 / 11 / 18$ & $20: 49: 49.641$ & 33.186 & -115.614 & 2.4 \\
$2009 / 11 / 18$ & $20: 56: 41.641$ & 33.181 & -115.603 & 1.4 \\
$2009 / 11 / 18$ & $21: 22: 50.641$ & 33.172 & -115.609 & 0.1 \\
$2009 / 11 / 18$ & $23: 13: 52.641$ & 33.173 & -115.609 & 0.1 \\
$2009 / 11 / 18$ & $00: 00: 55.641$ & 33.192 & -115.579 & 4.3 \\
$2009 / 11 / 19$ & $01: 17: 33.641$ & 33.176 & -115.609 & 2.1 \\
$2009 / 11 / 19$ & $01: 39: 19.641$ & 33.172 & -115.609 & 0.1 \\
$2009 / 11 / 19$ & $04: 41: 39.641$ & 33.190 & -115.608 & 2.2 \\
$2009 / 11 / 19$ & $05: 52: 05.641$ & 33.186 & -115.614 & 2.4 \\
$2009 / 11 / 19$ & $06: 42: 32.641$ & 33.179 & -115.594 & 1.5 \\
$2009 / 11 / 19$ & $06: 49: 19.641$ & 33.193 & -115.615 & 3.9 \\
$2009 / 11 / 19$ & $07: 16: 03.641$ & 33.176 & -115.608 & 0.4 \\
$2009 / 11 / 19$ & $08: 10: 11.641$ & 33.196 & -115.587 & 5.3 \\
$2009 / 11 / 19$ & $08: 12: 09.641$ & 33.181 & -115.665 & 4.2 \\
$2009 / 11 / 19$ & $08: 21: 23.641$ & 33.181 & -115.665 & 4.2 \\
$2009 / 11 / 19$ & $08: 35: 36.641$ & 33.186 & -115.604 & 2.0 \\
$2009 / 11 / 19$ & $09: 30: 21.641$ & 33.184 & -115.605 & 0.1 \\
$2009 / 11 / 19$ & $10: 18: 41.641$ & 33.184 & -115.605 & 0.1 \\
$2009 / 11 / 19$ & $12: 04: 48.641$ & 33.184 & -115.605 & 0.1 \\
$2009 / 11 / 19$ & $12: 07: 29.641$ & 33.184 & -115.605 & 0.1 \\
$2009 / 11 / 19$ & $13: 07: 31.641$ & 33.184 & -115.605 & 0.1 \\
$2009 / 11 / 19$ & $13: 35: 09.641$ & 33.184 & -115.605 & 0.1 \\
$2009 / 11 / 19$ & $21: 38: 36.641$ & 33.197 & -115.614 & 5.3 \\
$2009 / 11 / 18$ & $00: 00: 55.641$ & 33.192 & -115.579 & 4.3 \\
$2009 / 11 / 20$ & $00: 31: 30.641$ & 33.184 & -115.605 & 0.1 \\
$2009 / 11 / 20$ & $04: 22: 23.641$ & 33.229 & -115.645 & 5.7 \\
$2009 / 11 / 20$ & $05: 56: 09.641$ & 33.177 & -115.621 & 2.7 \\
$2009 / 11 / 20$ & $07: 24: 18.641$ & 33.177 & -115.635 & 5.3 \\
$2009 / 11 / 20$ & $09: 28: 57.641$ & 33.171 & -115.605 & 0.7 \\
$2009 / 11 / 20$ & $10: 24: 07.641$ & 33.108 & -115.602 & 8.1 \\
$2009 / 11 / 20$ & $14: 29: 40.641$ & 33.208 & -115.582 & 2.5 \\
\hline & $16: 38: 36.641$ & 33.171 & -115.605 & 0.7 \\
20 & $18: 32: 05.641$ & 33.202 & -115.575 & 3.8 \\
$20: 44: 56.641$ & 33.177 & -115.606 & 0.1
\end{tabular}




$\begin{array}{lllll}2009 / 11 / 20 & 21: 16: 50.641 & 33.108 & -115.602 & 8.1 \\ 2009 / 11 / 20 & 21: 53: 53.641 & 33.214 & -115.620 & 5.3 \\ 2009 / 11 / 20 & 23: 11: 56.641 & 33.118 & -115.610 & 9.1 \\ 2009 / 11 / 18 & 00: 00: 55.641 & 33.192 & -115.579 & 4.3 \\ 2009 / 11 / 21 & 01: 42: 04.641 & 33.173 & -115.610 & 1.3 \\ 2009 / 11 / 21 & 17: 12: 32.641 & 33.172 & -115.610 & 2.2 \\ 2009 / 11 / 21 & 17: 33: 31.641 & 33.159 & -115.641 & 0.9 \\ 2009 / 11 / 21 & 19: 13: 36.641 & 33.193 & -115.615 & 3.9 \\ 2009 / 11 / 18 & 00: 00: 55.641 & 33.192 & -115.579 & 4.3 \\ 2009 / 11 / 22 & 00: 05: 48.641 & 33.161 & -115.651 & 0.7 \\ 2009 / 11 / 22 & 00: 05: 49.641 & 33.189 & -115.576 & 0.1 \\ 2009 / 11 / 22 & 03: 27: 41.641 & 33.186 & -115.614 & 2.4 \\ 2009 / 11 / 22 & 05: 20: 47.641 & 33.149 & -115.640 & 3.5 \\ 2009 / 11 / 22 & 05: 27: 06.641 & 33.142 & -115.628 & 10.3 \\ 2009 / 11 / 22 & 05: 41: 14.641 & 33.118 & -115.610 & 9.1 \\ 2009 / 11 / 22 & 09: 01: 32.641 & 33.171 & -115.605 & 0.7 \\ 2009 / 11 / 22 & 09: 03: 49.641 & 33.197 & -115.614 & 5.3 \\ 2009 / 11 / 22 & 10: 27: 10.641 & 33.190 & -115.604 & 1.2 \\ 2009 / 11 / 22 & 12: 47: 48.641 & 33.179 & -115.594 & 1.5 \\ 2009 / 11 / 22 & 15: 39: 37.641 & 33.192 & -115.626 & 7.7 \\ 2009 / 11 / 22 & 17: 25: 51.641 & 33.149 & -115.640 & 3.5 \\ 2009 / 11 / 22 & 17: 51: 52.641 & 33.159 & -115.650 & 1.5 \\ 2009 / 11 / 22 & 18: 19: 08.641 & 33.189 & -115.605 & 1.5 \\ 2009 / 11 / 22 & 18: 19: 44.641 & 33.176 & -115.608 & 0.4 \\ 2009 / 11 / 22 & 18: 54: 05.641 & 33.183 & -115.617 & 4.3 \\ 2009 / 11 / 22 & 21: 33: 07.641 & 33.184 & -115.608 & 0.3 \\ 2009 / 11 / 23 & 00: 00: 44.641 & 33.193 & -115.632 & 3.5 \\ 2009 / 11 / 23 & 02: 20: 34.641 & 33.108 & -115.602 & 8.1 \\ 2009 / 11 / 23 & 12: 43: 30.641 & 33.183 & -115.677 & 0.5 \\ 2009 / 11 / 23 & 12: 47: 24.641 & 33.165 & -115.623 & 7.0 \\ 2009 / 11 / 23 & 12: 50: 55.641 & 33.183 & -115.677 & 0.5 \\ 2009 / 11 / 23 & 13: 13: 49.641 & 33.140 & -115.636 & 7.3 \\ 2009 / 11 / 23 & 13: 21: 54.641 & 33.130 & -115.634 & 0.0 \\ 2009 / 11 / 23 & 14: 21: 40.641 & 33.176 & -115.604 & 0.0 \\ 2009 / 11 / 23 & 20: 00: 56.641 & 33.176 & -115.609 & 2.1 \\ 2009 / 11 / 23 & 22: 59: 52.641 & 33.184 & -115.605 & 0.1 \\ 2009 / 11 / 18 & 00: 00: 55.641 & 33.192 & -115.579 & 4.3 \\ 2009 / 11 / 24 & 00: 04: 47.641 & 33.184 & -115.605 & 0.1 \\ 2009 / 11 / 24 & 00: 38: 24.641 & 33.158 & -115.629 & 6.0 \\ 2009 / 11 / 24 & 00: 42: 30.641 & 33.184 & -115.605 & 0.1 \\ 2009 / 11 / 24 & 02: 43: 34.641 & 33.218 & -115.639 & 3.0 \\ 2009 / 11 / 24 & 04: 26: 33.641 & 33.181 & -115.665 & 4.2 \\ 2009 / 11 / 24 & 04: 27: 16.641 & 33.181 & -115.665 & 4.2 \\ 2009 / 11 / 24 & 04: 27: 39.641 & 33.181 & -115.665 & 4.2 \\ 2009 / 11 / 24 & 04: 28: 31.641 & 33.158 & -115.629 & 6.0 \\ 2009 / 11 / 24 & 04: 31: 55.641 & 33.158 & -115.629 & 6.0\end{array}$




$\begin{array}{lllll}2009 / 11 / 24 & 04: 34: 38.641 & 33.226 & -115.588 & 4.6 \\ 2009 / 11 / 24 & 04: 35: 17.641 & 33.181 & -115.665 & 4.2 \\ 2009 / 11 / 24 & 04: 36: 29.641 & 33.181 & -115.665 & 4.2 \\ 2009 / 11 / 24 & 04: 37: 15.641 & 33.181 & -115.665 & 4.2 \\ 2009 / 11 / 24 & 04: 37: 38.641 & 33.181 & -115.665 & 4.2 \\ 2009 / 11 / 24 & 04: 37: 52.641 & 33.181 & -115.665 & 4.2 \\ 2009 / 11 / 24 & 04: 38: 47.641 & 33.181 & -115.665 & 4.2 \\ 2009 / 11 / 24 & 04: 39: 55.641 & 33.176 & -115.618 & 3.7 \\ 2009 / 11 / 24 & 04: 40: 39.641 & 33.181 & -115.665 & 4.2 \\ 2009 / 11 / 24 & 04: 41: 40.641 & 33.181 & -115.665 & 4.2 \\ 2009 / 11 / 24 & 04: 44: 37.641 & 33.181 & -115.665 & 4.2 \\ 2009 / 11 / 24 & 04: 44: 52.641 & 33.176 & -115.609 & 2.1 \\ 2009 / 11 / 24 & 04: 46: 15.641 & 33.176 & -115.609 & 2.1 \\ 2009 / 11 / 24 & 04: 46: 50.641 & 33.181 & -115.665 & 4.2 \\ 2009 / 11 / 24 & 04: 49: 44.641 & 33.181 & -115.665 & 4.2 \\ 2009 / 11 / 24 & 04: 51: 08.641 & 33.190 & -115.608 & 2.2 \\ 2009 / 11 / 24 & 04: 53: 36.641 & 33.176 & -115.609 & 2.1 \\ 2009 / 11 / 24 & 05: 00: 45.641 & 33.182 & -115.603 & 0.1 \\ 2009 / 11 / 24 & 05: 01: 43.641 & 33.181 & -115.665 & 4.2 \\ 2009 / 11 / 24 & 05: 03: 36.641 & 33.165 & -115.623 & 7.0 \\ 2009 / 11 / 24 & 05: 05: 45.641 & 33.181 & -115.665 & 4.2 \\ 2009 / 11 / 24 & 05: 08: 36.641 & 33.181 & -115.665 & 4.2 \\ 2009 / 11 / 24 & 05: 08: 59.641 & 33.248 & -115.633 & 2.9 \\ 2009 / 11 / 24 & 05: 13: 12.641 & 33.190 & -115.604 & 1.2 \\ 2009 / 11 / 24 & 05: 32: 32.641 & 33.176 & -115.609 & 2.1 \\ 2009 / 11 / 24 & 05: 32: 50.641 & 33.181 & -115.665 & 4.2 \\ 2009 / 11 / 24 & 05: 40: 04.641 & 33.165 & -115.623 & 7.0 \\ 2009 / 11 / 24 & 05: 41: 01.641 & 33.165 & -115.623 & 7.0 \\ 2009 / 11 / 24 & 05: 41: 22.641 & 33.165 & -115.623 & 7.0 \\ 2009 / 11 / 24 & 05: 44: 08.641 & 33.165 & -115.623 & 7.0 \\ 2009 / 11 / 24 & 05: 44: 33.641 & 33.181 & -115.665 & 4.2 \\ 2009 / 11 / 24 & 05: 46: 07.641 & 33.165 & -115.623 & 7.0 \\ 2009 / 11 / 24 & 06: 09: 35.641 & 33.165 & -115.623 & 7.0 \\ 2009 / 11 / 24 & 06: 15: 49.641 & 33.181 & -115.665 & 4.2 \\ 2009 / 11 / 24 & 06: 29: 03.641 & 33.161 & -115.651 & 0.7 \\ 2009 / 11 / 24 & 06: 29: 44.641 & 33.161 & -115.651 & 0.7 \\ 2009 / 11 / 24 & 06: 30: 49.641 & 33.161 & -115.651 & 0.7 \\ 2009 / 11 / 24 & 09: 54: 30.641 & 33.182 & -115.603 & 0.1 \\ 2009 / 11 / 24 & 09: 54: 46.641 & 33.176 & -115.608 & 0.4 \\ 2009 / 11 / 24 & 10: 46: 29.641 & 33.181 & -115.603 & 0.1 \\ 2009 / 11 / 24 & 10: 59: 39.641 & 33.181 & -115.603 & 0.1 \\ 2009 / 11 / 24 & 11: 00: 03.641 & 33.181 & -115.603 & 0.1 \\ 2009 / 11 / 24 & 11: 00: 54.641 & 33.181 & -115.603 & 0.1 \\ 2009 / 11 / 24 & 11: 02: 10.641 & 33.181 & -115.603 & 0.1 \\ 2009 / 11 / 24 & 11: 10: 19.641 & 33.188 & -115.600 & 0.1\end{array}$




$\begin{array}{lllll}2009 / 11 / 24 & 11: 10: 46.641 & 33.188 & -115.600 & 0.1 \\ 2009 / 11 / 24 & 11: 19: 46.641 & 33.188 & -115.600 & 0.1 \\ 2009 / 11 / 24 & 11: 20: 29.641 & 33.158 & -115.629 & 6.0 \\ 2009 / 11 / 24 & 11: 32: 43.641 & 33.176 & -115.608 & 0.4 \\ 2009 / 11 / 24 & 11: 33: 15.641 & 33.179 & -115.594 & 1.5 \\ 2009 / 11 / 24 & 11: 42: 06.641 & 33.181 & -115.602 & 0.4 \\ 2009 / 11 / 24 & 11: 59: 14.641 & 33.185 & -115.608 & 2.0 \\ 2009 / 11 / 24 & 12: 00: 04.641 & 33.188 & -115.600 & 0.1 \\ 2009 / 11 / 24 & 12: 04: 00.641 & 33.176 & -115.608 & 0.4 \\ 2009 / 11 / 24 & 12: 20: 53.641 & 33.182 & -115.618 & 3.1 \\ 2009 / 11 / 24 & 12: 27: 20.641 & 33.158 & -115.629 & 6.0 \\ 2009 / 11 / 24 & 12: 30: 24.641 & 33.182 & -115.612 & 2.2 \\ 2009 / 11 / 24 & 12: 31: 17.641 & 33.188 & -115.609 & 2.3 \\ 2009 / 11 / 24 & 12: 40: 31.641 & 33.185 & -115.608 & 2.0 \\ 2009 / 11 / 24 & 12: 42: 02.641 & 33.185 & -115.608 & 2.0 \\ 2009 / 11 / 24 & 12: 47: 57.641 & 33.181 & -115.603 & 0.1 \\ 2009 / 11 / 24 & 13: 09: 03.641 & 33.185 & -115.608 & 2.0 \\ 2009 / 11 / 24 & 13: 12: 30.641 & 33.181 & -115.665 & 4.2 \\ 2009 / 11 / 24 & 13: 17: 13.641 & 33.182 & -115.618 & 3.1 \\ 2009 / 11 / 24 & 13: 22: 27.641 & 33.185 & -115.608 & 2.0 \\ 2009 / 11 / 24 & 13: 26: 52.641 & 33.181 & -115.602 & 0.4 \\ 2009 / 11 / 24 & 13: 39: 57.641 & 33.185 & -115.608 & 2.0 \\ 2009 / 11 / 24 & 13: 44: 17.641 & 33.185 & -115.608 & 2.0 \\ 2009 / 11 / 24 & 13: 44: 33.641 & 33.181 & -115.602 & 0.4 \\ 2009 / 11 / 24 & 13: 48: 23.641 & 33.181 & -115.602 & 0.4 \\ 2009 / 11 / 24 & 14: 33: 16.641 & 33.181 & -115.602 & 0.4 \\ 2009 / 11 / 24 & 14: 38: 56.641 & 33.176 & -115.608 & 0.4 \\ 2009 / 11 / 24 & 14: 47: 47.641 & 33.184 & -115.610 & 2.8 \\ 2009 / 11 / 24 & 15: 21: 12.641 & 33.184 & -115.610 & 2.8 \\ 2009 / 11 / 24 & 15: 59: 13.641 & 33.188 & -115.600 & 0.1 \\ 2009 / 11 / 24 & 16: 33: 00.641 & 33.176 & -115.608 & 0.4 \\ 2009 / 11 / 24 & 16: 48: 27.641 & 33.187 & -115.604 & 3.2 \\ 2009 / 11 / 24 & 16: 49: 30.641 & 33.185 & -115.608 & 2.0 \\ 2009 / 11 / 24 & 16: 49: 45.641 & 33.185 & -115.608 & 2.0 \\ 2009 / 11 / 24 & 16: 51: 41.641 & 33.140 & -115.636 & 7.3 \\ 2009 / 11 / 24 & 16: 58: 58.641 & 33.186 & -115.612 & 0.6 \\ 2009 / 11 / 24 & 17: 07: 08.641 & 33.185 & -115.608 & 2.0 \\ 2009 / 11 / 24 & 17: 15: 31.641 & 33.185 & -115.608 & 2.0 \\ 2009 / 11 / 24 & 17: 41: 31.641 & 33.181 & -115.602 & 0.4 \\ 2009 / 11 / 24 & 18: 12: 35.641 & 33.181 & -115.614 & 3.1 \\ 2009 / 11 / 24 & 18: 41: 18.641 & 33.185 & -115.608 & 2.0 \\ 2009 / 11 / 24 & 19: 07: 18.641 & 33.164 & -115.640 & 3.4 \\ 2009 / 11 / 24 & 19: 08: 27.641 & 33.118 & -115.610 & 9.1 \\ 2009 / 11 / 24 & 19: 17: 16.641 & 33.185 & -115.608 & 2.0 \\ 2009 / 11 / 24 & 19: 18: 27.641 & 33.185 & -115.608 & 2.0 \\ & 19: 21: 08.641 & 33.185 & -115.608 & 2.0\end{array}$




$\begin{array}{lllll}2009 / 11 / 24 & 19: 21: 30.641 & 33.185 & -115.608 & 2.0 \\ 2009 / 11 / 24 & 19: 24: 01.641 & 33.201 & -115.544 & 3.4 \\ 2009 / 11 / 24 & 19: 27: 32.641 & 33.185 & -115.608 & 2.0 \\ 2009 / 11 / 24 & 19: 30: 16.641 & 33.185 & -115.608 & 2.0 \\ 2009 / 11 / 24 & 19: 43: 34.641 & 33.185 & -115.608 & 2.0 \\ 2009 / 11 / 24 & 19: 44: 06.641 & 33.185 & -115.608 & 2.0 \\ 2009 / 11 / 24 & 19: 48: 46.641 & 33.181 & -115.665 & 4.2 \\ 2009 / 11 / 24 & 21: 01: 20.641 & 33.181 & -115.665 & 4.2 \\ 2009 / 11 / 24 & 21: 03: 12.641 & 33.181 & -115.665 & 4.2 \\ 2009 / 11 / 24 & 21: 47: 35.641 & 33.181 & -115.665 & 4.2 \\ 2009 / 11 / 24 & 21: 50: 40.641 & 33.158 & -115.629 & 6.0 \\ 2009 / 11 / 24 & 22: 02: 41.641 & 33.108 & -115.602 & 8.1 \\ 2009 / 11 / 24 & 22: 13: 47.641 & 33.179 & -115.602 & 0.6 \\ 2009 / 11 / 24 & 22: 15: 34.641 & 33.179 & -115.602 & 0.6 \\ 2009 / 11 / 24 & 23: 26: 13.641 & 33.184 & -115.610 & 2.8 \\ 2009 / 11 / 18 & 00: 00: 56.641 & 33.192 & -115.579 & 4.3 \\ 2009 / 11 / 25 & 00: 17: 51.641 & 33.171 & -115.605 & 0.7 \\ 2009 / 11 / 25 & 00: 47: 50.641 & 33.157 & -115.641 & 2.2 \\ 2009 / 11 / 25 & 00: 48: 49.641 & 33.157 & -115.641 & 2.2 \\ 2009 / 11 / 25 & 00: 49: 04.641 & 33.157 & -115.641 & 2.2 \\ 2009 / 11 / 25 & 00: 50: 15.641 & 33.157 & -115.641 & 2.2 \\ 2009 / 11 / 25 & 00: 51: 21.641 & 33.157 & -115.641 & 2.2 \\ 2009 / 11 / 25 & 00: 52: 16.641 & 33.157 & -115.641 & 2.2 \\ 2009 / 11 / 25 & 02: 01: 03.641 & 33.193 & -115.615 & 3.9 \\ 2009 / 11 / 25 & 02: 24: 16.641 & 33.182 & -115.612 & 2.2 \\ 2009 / 11 / 25 & 02: 59: 36.641 & 33.185 & -115.608 & 2.0 \\ 2009 / 11 / 25 & 04: 59: 59.641 & 33.176 & -115.602 & 3.2 \\ 2009 / 11 / 25 & 05: 36: 04.641 & 33.159 & -115.640 & 1.5 \\ 2009 / 11 / 25 & 05: 41: 14.641 & 33.176 & -115.604 & 0.5 \\ 2009 / 11 / 25 & 05: 58: 25.641 & 33.159 & -115.640 & 1.5 \\ 2009 / 11 / 25 & 06: 32: 06.641 & 33.108 & -115.602 & 8.1 \\ 2009 / 11 / 25 & 06: 51: 48.641 & 33.185 & -115.608 & 2.0 \\ 2009 / 11 / 25 & 07: 58: 46.641 & 33.185 & -115.608 & 2.0 \\ 2009 / 11 / 25 & 13: 51: 54.641 & 33.185 & -115.608 & 2.0 \\ 2009 / 11 / 25 & 20: 24: 54.641 & 33.176 & -115.609 & 2.1 \\ 2009 / 11 / 25 & 22: 04: 43.641 & 33.187 & -115.601 & 1.7 \\ 2009 / 11 / 18 & 00: 00: 55.641 & 33.192 & -115.579 & 4.3 \\ 2009 / 11 / 26 & 01: 15: 15.641 & 33.181 & -115.602 & 0.4 \\ 2009 / 11 / 26 & 03: 55: 19.641 & 33.182 & -115.602 & 2.5 \\ 2009 / 11 / 26 & 04: 17: 59.641 & 33.181 & -115.602 & 0.4 \\ 2009 / 11 / 26 & 05: 33: 31.641 & 33.181 & -115.602 & 0.4 \\ 2009 / 11 / 26 & 05: 35: 26.641 & 33.186 & -115.614 & 2.4 \\ 2009 / 11 / 26 & 05: 57: 36.641 & 33.175 & -115.603 & 0.1 \\ 2009 / 11 / 26 & 06: 36: 46.641 & 33.179 & -115.597 & 0.1 \\ 209: 08: 55.641 & 33.181 & -115.602 & 0.4 \\ 26 & 10: 44: 51.641 & 33.202 & -115.671 & 6.3\end{array}$




$\begin{array}{lllll}2009 / 11 / 26 & 14: 14: 41.641 & 33.157 & -115.641 & 2.2 \\ 2009 / 11 / 26 & 16: 41: 06.641 & 33.176 & -115.608 & 0.4 \\ 2009 / 11 / 26 & 17: 52: 30.641 & 33.171 & -115.605 & 0.7 \\ 2009 / 11 / 26 & 17: 54: 43.641 & 33.178 & -115.611 & 1.6 \\ 2009 / 11 / 26 & 17: 56: 12.641 & 33.171 & -115.605 & 0.7 \\ 2009 / 11 / 26 & 18: 26: 43.641 & 33.171 & -115.605 & 0.7 \\ 2009 / 11 / 26 & 18: 36: 16.641 & 33.171 & -115.605 & 0.7 \\ 2009 / 11 / 26 & 18: 38: 58.641 & 33.171 & -115.605 & 0.7 \\ 2009 / 11 / 26 & 22: 54: 26.641 & 33.181 & -115.601 & 0.7 \\ 2009 / 11 / 26 & 23: 03: 47.641 & 33.192 & -115.626 & 7.7 \\ 2009 / 11 / 26 & 23: 06: 24.641 & 33.118 & -115.610 & 9.1 \\ 2009 / 11 / 26 & 23: 14: 39.641 & 33.202 & -115.575 & 3.8 \\ 2009 / 11 / 26 & 23: 29: 50.641 & 33.115 & -115.585 & 6.5 \\ 2009 / 11 / 18 & 00: 00: 55.641 & 33.192 & -115.579 & 4.3 \\ 2009 / 11 / 27 & 00: 06: 14.641 & 33.229 & -115.645 & 5.7 \\ 2009 / 11 / 27 & 00: 44: 42.641 & 33.186 & -115.614 & 2.4 \\ 2009 / 11 / 27 & 05: 22: 56.641 & 33.161 & -115.651 & 0.7 \\ 2009 / 11 / 27 & 07: 45: 50.641 & 33.171 & -115.605 & 0.7 \\ 2009 / 11 / 27 & 08: 07: 29.641 & 33.185 & -115.608 & 2.0 \\ 2009 / 11 / 27 & 09: 08: 13.641 & 33.179 & -115.602 & 0.6 \\ 2009 / 11 / 27 & 09: 37: 31.641 & 33.179 & -115.594 & 1.5 \\ 2009 / 11 / 27 & 14: 59: 41.641 & 33.199 & -115.617 & 0.0 \\ 2009 / 11 / 27 & 15: 25: 41.641 & 33.199 & -115.617 & 0.0 \\ 2009 / 11 / 27 & 15: 52: 16.641 & 33.199 & -115.617 & 0.0 \\ 2009 / 11 / 27 & 17: 53: 43.641 & 33.202 & -115.575 & 3.8 \\ 2009 / 11 / 27 & 18: 41: 11.641 & 33.159 & -115.641 & 0.9 \\ 2009 / 11 / 27 & 20: 39: 02.641 & 33.188 & -115.573 & 1.2 \\ 2009 / 11 / 27 & 21: 32: 38.641 & 33.182 & -115.602 & 2.5 \\ 2009 / 11 / 27 & 22: 47: 39.641 & 33.208 & -115.582 & 2.5 \\ 2009 / 11 / 28 & 03: 59: 11.641 & 33.187 & -115.604 & 3.2 \\ 2009 / 11 / 28 & 04: 47: 28.641 & 33.181 & -115.601 & 0.7 \\ 2009 / 11 / 28 & 07: 24: 53.641 & 33.214 & -115.620 & 5.3 \\ 2009 / 11 / 28 & 11: 28: 58.641 & 33.179 & -115.594 & 1.5 \\ 2009 / 11 / 28 & 11: 35: 41.641 & 33.176 & -115.608 & 0.4 \\ 2009 / 11 / 28 & 15: 33: 17.641 & 33.234 & -115.604 & 4.8 \\ 2009 / 11 / 28 & 15: 45: 15.641 & 33.184 & -115.609 & 2.2 \\ 2009 / 11 / 28 & 18: 01: 40.641 & 33.194 & -115.583 & 3.0 \\ 2009 / 11 / 28 & 18: 19: 47.641 & 33.194 & -115.583 & 3.0 \\ 2009 / 11 / 28 & 21: 40: 35.641 & 33.192 & -115.626 & 7.7 \\ 2009 / 11 / 28 & 21: 41: 51.641 & 33.180 & -115.609 & 0.0 \\ 2009 / 11 / 28 & 21: 43: 18.641 & 33.192 & -115.626 & 7.7 \\ 2009 / 11 / 28 & 21: 47: 14.641 & 33.182 & -115.606 & 1.7 \\ 2009 / 11 / 28 & 23: 03: 57.641 & 33.184 & -115.640 & 3.5 \\ 23: 11 / 28 & 23: 26: 45.641 & 33.202 & -115.605 & 0.1 \\ 29 & 00: 06: 08.641 & 33.193 & -115.615 & 3.9\end{array}$




$\begin{array}{lllll}2009 / 11 / 29 & 04: 07: 34.641 & 33.159 & -115.640 & 1.5 \\ 2009 / 11 / 29 & 04: 10: 48.641 & 33.158 & -115.638 & 0.4 \\ 2009 / 11 / 29 & 04: 11: 17.641 & 33.158 & -115.638 & 0.4 \\ 2009 / 11 / 29 & 04: 12: 35.641 & 33.135 & -115.644 & 6.9 \\ 2009 / 11 / 29 & 04: 20: 35.641 & 33.158 & -115.638 & 0.4 \\ 2009 / 11 / 29 & 06: 02: 41.641 & 33.140 & -115.636 & 7.3 \\ 2009 / 11 / 29 & 06: 44: 46.641 & 33.195 & -115.572 & 0.1 \\ 2009 / 11 / 29 & 06: 55: 53.641 & 33.195 & -115.572 & 0.1 \\ 2009 / 11 / 29 & 06: 59: 51.641 & 33.184 & -115.605 & 0.1 \\ 2009 / 11 / 29 & 07: 00: 34.641 & 33.184 & -115.605 & 0.1 \\ 2009 / 11 / 29 & 08: 14: 41.641 & 33.196 & -115.587 & 5.3 \\ 2009 / 11 / 29 & 12: 52: 31.641 & 33.181 & -115.611 & 0.4 \\ 2009 / 11 / 29 & 12: 54: 21.641 & 33.181 & -115.611 & 0.4 \\ 2009 / 11 / 29 & 13: 10: 57.641 & 33.186 & -115.612 & 0.6 \\ 2009 / 11 / 29 & 13: 12: 48.641 & 33.186 & -115.612 & 0.6 \\ 2009 / 11 / 29 & 13: 13: 03.641 & 33.182 & -115.603 & 0.1 \\ 2009 / 11 / 29 & 13: 13: 58.641 & 33.182 & -115.603 & 0.1 \\ 2009 / 11 / 29 & 13: 19: 36.641 & 33.231 & -115.605 & 4.8 \\ 2009 / 11 / 29 & 13: 44: 46.641 & 33.165 & -115.623 & 7.0 \\ 2009 / 11 / 29 & 16: 28: 22.641 & 33.184 & -115.597 & 0.1 \\ 2009 / 11 / 29 & 19: 06: 39.641 & 33.193 & -115.615 & 3.9 \\ 2009 / 11 / 30 & 02: 05: 57.641 & 33.186 & -115.593 & 0.1 \\ 2009 / 11 / 30 & 02: 19: 07.641 & 33.142 & -115.628 & 10.3 \\ 2009 / 11 / 30 & 02: 19: 41.641 & 33.159 & -115.641 & 0.9 \\ 2009 / 11 / 30 & 02: 19: 57.641 & 33.158 & -115.642 & 0.1 \\ 2009 / 11 / 30 & 02: 20: 16.641 & 33.158 & -115.642 & 0.1 \\ 2009 / 11 / 30 & 02: 21: 41.641 & 33.159 & -115.641 & 0.9 \\ 2009 / 11 / 30 & 02: 21: 55.641 & 33.130 & -115.634 & 0.0 \\ 2009 / 11 / 30 & 02: 24: 05.641 & 33.140 & -115.636 & 7.3 \\ 2009 / 11 / 30 & 02: 56: 45.641 & 33.108 & -115.602 & 8.1 \\ 2009 / 11 / 30 & 06: 15: 42.641 & 33.159 & -115.640 & 1.5 \\ 2009 / 11 / 30 & 11: 22: 59.641 & 33.231 & -115.605 & 4.8 \\ 2009 / 11 / 30 & 11: 42: 03.641 & 33.187 & -115.604 & 3.2 \\ 2009 / 11 / 30 & 12: 04: 31.641 & 33.135 & -115.644 & 6.9 \\ 2009 / 11 / 30 & 12: 53: 07.641 & 33.187 & -115.604 & 3.2 \\ 2009 / 12 / 01 & 22: 45: 14.641 & 33.175 & -115.600 & 1.1 \\ 2009 / 12 / 01 & 22: 46: 55.641 & 33.181 & -115.659 & 6.0 \\ 2009 / 12 / 01 & 22: 47: 39.641 & 33.179 & -115.614 & 1.8 \\ 2009 / 12 / 01 & 22: 48: 02.641 & 33.187 & -115.608 & 0.2 \\ 2009 / 12 / 01 & 22: 54: 34.641 & 33.229 & -115.576 & 3.4 \\ 2009 / 12 / 01 & 22: 54: 47.641 & 33.208 & -115.582 & 2.5 \\ 2009 / 12 / 01 & 22: 59: 33.641 & 33.177 & -115.606 & 0.1 \\ 2009 / 12 / 01 & 23: 12: 34.641 & 33.184 & -115.604 & 0.1 \\ 2009 / 12 / 01 & 23: 13: 44.641 & 33.184 & -115.604 & 0.1 \\ 2009 / 12 / 01 & 23: 16: 01.641 & 33.179 & -115.614 & 1.8\end{array}$




$\begin{array}{lllll}2009 / 12 / 01 & 23: 18: 02.641 & 33.179 & -115.614 & 1.8 \\ 2009 / 12 / 01 & 23: 18: 36.641 & 33.187 & -115.608 & 0.2 \\ 2009 / 12 / 01 & 23: 22: 02.641 & 33.179 & -115.614 & 1.8 \\ 2009 / 12 / 01 & 23: 22: 38.641 & 33.176 & -115.604 & 0.0 \\ 2009 / 12 / 01 & 23: 30: 27.641 & 33.179 & -115.614 & 1.8 \\ 2009 / 12 / 01 & 23: 30: 39.641 & 33.199 & -115.571 & 3.3 \\ 2009 / 12 / 01 & 23: 31: 57.641 & 33.179 & -115.614 & 1.8 \\ 2009 / 12 / 01 & 23: 34: 39.641 & 33.181 & -115.665 & 4.2 \\ 2009 / 12 / 01 & 23: 41: 31.641 & 33.179 & -115.614 & 1.8 \\ 2009 / 12 / 02 & 03: 13: 04.641 & 33.118 & -115.590 & 13.8 \\ 2009 / 12 / 02 & 07: 08: 06.641 & 33.181 & -115.665 & 4.2 \\ 2009 / 12 / 02 & 07: 09: 01.641 & 33.181 & -115.665 & 4.2 \\ 2009 / 12 / 02 & 07: 09: 29.641 & 33.181 & -115.665 & 4.2 \\ 2009 / 12 / 02 & 07: 11: 25.641 & 33.179 & -115.621 & 4.8 \\ 2009 / 12 / 02 & 07: 11: 58.641 & 33.184 & -115.611 & 2.7 \\ 2009 / 12 / 02 & 07: 12: 55.641 & 33.181 & -115.601 & 0.7 \\ 2009 / 12 / 02 & 07: 36: 15.641 & 33.181 & -115.665 & 4.2 \\ 2009 / 12 / 02 & 07: 36: 54.641 & 33.193 & -115.583 & 5.9 \\ 2009 / 12 / 02 & 07: 40: 13.641 & 33.181 & -115.665 & 4.2 \\ 2009 / 12 / 02 & 07: 42: 25.641 & 33.179 & -115.614 & 1.8 \\ 2009 / 12 / 02 & 07: 45: 52.641 & 33.177 & -115.621 & 2.7 \\ 2009 / 12 / 02 & 11: 23: 00.641 & 33.108 & -115.602 & 8.1 \\ 2009 / 12 / 02 & 13: 02: 46.641 & 33.231 & -115.657 & 2.0 \\ 2009 / 12 / 02 & 13: 04: 04.641 & 33.181 & -115.659 & 6.0 \\ 2009 / 12 / 02 & 13: 07: 09.641 & 33.213 & -115.584 & 1.3 \\ 2009 / 12 / 02 & 13: 19: 03.641 & 33.231 & -115.657 & 2.0 \\ 2009 / 12 / 03 & 06: 07: 28.641 & 33.184 & -115.608 & 0.3 \\ 2009 / 12 / 03 & 07: 46: 50.641 & 33.179 & -115.614 & 1.8 \\ 2009 / 12 / 03 & 07: 47: 40.641 & 33.179 & -115.614 & 1.8 \\ 2009 / 12 / 03 & 07: 51: 09.641 & 33.179 & -115.614 & 1.8 \\ 2009 / 12 / 03 & 07: 53: 06.641 & 33.179 & -115.614 & 1.8 \\ 2009 / 12 / 03 & 11: 10: 20.641 & 33.196 & -115.618 & 7.3 \\ 2009 / 12 / 03 & 11: 15: 53.641 & 33.218 & -115.639 & 3.0 \\ 2009 / 12 / 03 & 12: 05: 34.641 & 33.179 & -115.594 & 1.5 \\ 2009 / 12 / 03 & 12: 50: 20.641 & 33.196 & -115.587 & 4.5 \\ 2009 / 12 / 03 & 13: 12: 28.641 & 33.196 & -115.587 & 4.5 \\ 2009 / 12 / 03 & 21: 09: 14.641 & 33.183 & -115.607 & 0.0 \\ 2009 / 12 / 04 & 03: 02: 13.641 & 33.140 & -115.636 & 7.3 \\ 2009 / 12 / 04 & 07: 45: 49.641 & 33.117 & -115.613 & 7.9 \\ 2009 / 12 / 04 & 09: 28: 25.641 & 33.199 & -115.570 & 0.2 \\ 2009 / 12 / 04 & 11: 36: 05.641 & 33.176 & -115.608 & 0.4 \\ 2009 / 12 / 04 & 12: 54: 38.641 & 33.158 & -115.644 & 0.1 \\ 2009 / 12 / 04 & 13: 56: 37.641 & 33.190 & -115.604 & 1.2 \\ 2009 / 12 / 04 & 14: 00: 45.641 & 33.190 & -115.604 & 1.2 \\ 2009 / 12 / 04 & 14: 01: 00.641 & 33.190 & -115.604 & 1.2 \\ 2009 / 12 / 04 & 14: 28: 57.641 & 33.190 & -115.604 & 1.2\end{array}$




$\begin{array}{lllll}2009 / 12 / 04 & 14: 40: 43.641 & 33.190 & -115.604 & 1.2 \\ 2009 / 12 / 04 & 14: 44: 00.641 & 33.190 & -115.604 & 1.2 \\ 2009 / 12 / 04 & 14: 45: 25.641 & 33.182 & -115.603 & 0.1 \\ 2009 / 12 / 04 & 20: 29: 08.641 & 33.187 & -115.604 & 3.2 \\ 2009 / 12 / 04 & 20: 33: 37.641 & 33.187 & -115.604 & 3.2 \\ 2009 / 12 / 04 & 22: 23: 05.641 & 33.108 & -115.602 & 8.1 \\ 2009 / 12 / 05 & 01: 34: 23.641 & 33.184 & -115.604 & 0.1 \\ 2009 / 12 / 05 & 01: 35: 06.641 & 33.184 & -115.604 & 0.1 \\ 2009 / 12 / 05 & 01: 43: 53.641 & 33.117 & -115.613 & 7.9 \\ 2009 / 12 / 05 & 02: 05: 17.641 & 33.187 & -115.604 & 3.2 \\ 2009 / 12 / 05 & 07: 37: 35.641 & 33.183 & -115.599 & 0.1 \\ 2009 / 12 / 05 & 07: 49: 00.641 & 33.236 & -115.573 & 5.0 \\ 2009 / 12 / 05 & 11: 45: 18.641 & 33.183 & -115.599 & 0.1 \\ 2009 / 12 / 05 & 12: 14: 47.641 & 33.183 & -115.599 & 0.1 \\ 2009 / 12 / 06 & 07: 37: 56.641 & 33.118 & -115.610 & 9.1 \\ 2009 / 12 / 06 & 08: 03: 06.641 & 33.145 & -115.634 & 4.8 \\ 2009 / 12 / 07 & 04: 02: 57.641 & 33.201 & -115.544 & 3.4 \\ 2009 / 12 / 07 & 04: 03: 30.641 & 33.201 & -115.544 & 3.4 \\ 2009 / 12 / 07 & 04: 08: 34.641 & 33.135 & -115.644 & 6.9 \\ 2009 / 12 / 07 & 04: 09: 30.641 & 33.176 & -115.606 & 6.3 \\ 2009 / 12 / 07 & 05: 11: 24.641 & 33.175 & -115.601 & 0.1 \\ 2009 / 12 / 07 & 07: 20: 01.641 & 33.179 & -115.594 & 1.5 \\ 2009 / 12 / 07 & 08: 40: 07.641 & 33.196 & -115.559 & 0.2 \\ 2009 / 12 / 07 & 11: 28: 36.641 & 33.198 & -115.571 & 0.2 \\ 2009 / 12 / 07 & 13: 01: 34.641 & 33.202 & -115.575 & 3.8 \\ 2009 / 12 / 07 & 13: 18: 31.641 & 33.184 & -115.605 & 0.1 \\ 2009 / 12 / 07 & 19: 22: 53.641 & 33.176 & -115.608 & 0.4 \\ 2009 / 12 / 07 & 20: 41: 53.641 & 33.177 & -115.606 & 0.1 \\ 2009 / 12 / 08 & 03: 02: 45.641 & 33.213 & -115.584 & 1.3 \\ 2009 / 12 / 08 & 03: 06: 07.641 & 33.213 & -115.584 & 1.3 \\ 2009 / 12 / 08 & 03: 07: 00.641 & 33.209 & -115.590 & 3.1 \\ 2009 / 12 / 08 & 03: 13: 22.641 & 33.209 & -115.590 & 3.1 \\ 2009 / 12 / 08 & 03: 20: 01.641 & 33.181 & -115.665 & 4.2 \\ 2009 / 12 / 08 & 04: 15: 00.641 & 33.208 & -115.631 & 5.0 \\ 2009 / 12 / 08 & 04: 44: 53.641 & 33.229 & -115.645 & 5.7 \\ 2009 / 12 / 08 & 04: 45: 04.641 & 33.236 & -115.573 & 5.0 \\ 2009 / 12 / 08 & 11: 11: 55.641 & 33.186 & -115.612 & 0.6 \\ 2009 / 12 / 08 & 12: 32: 25.641 & 33.194 & -115.583 & 3.0 \\ 2009 / 12 / 08 & 12: 53: 21.641 & 33.159 & -115.640 & 1.5 \\ 2009 / 12 / 08 & 12: 54: 04.641 & 33.159 & -115.640 & 1.5 \\ 2009 / 12 / 08 & 12: 54: 31.641 & 33.159 & -115.640 & 1.5 \\ 2009 / 12 / 08 & 12: 56: 43.641 & 33.159 & -115.640 & 1.5 \\ 2009 / 12 / 08 & 13: 00: 44.641 & 33.159 & -115.640 & 1.5 \\ 2 / 08 & 13: 04: 19.641 & 33.159 & -115.640 & 1.5 \\ 2: 11: 52.641 & 33.159 & -115.640 & 1.5\end{array}$




$\begin{array}{lllll}2009 / 12 / 08 & 13: 13: 01.641 & 33.159 & -115.640 & 1.5 \\ 2009 / 12 / 08 & 13: 40: 17.641 & 33.149 & -115.640 & 3.5 \\ 2009 / 12 / 08 & 13: 50: 31.641 & 33.159 & -115.640 & 1.5 \\ 2009 / 12 / 08 & 18: 14: 49.641 & 33.185 & -115.569 & 3.7 \\ 2009 / 12 / 08 & 18: 59: 37.641 & 33.182 & -115.602 & 2.5 \\ 2009 / 12 / 08 & 19: 00: 23.641 & 33.196 & -115.559 & 0.2 \\ 2009 / 12 / 08 & 20: 27: 16.641 & 33.185 & -115.569 & 3.7 \\ 2009 / 12 / 08 & 20: 31: 40.641 & 33.196 & -115.566 & 3.2 \\ 2009 / 12 / 08 & 21: 04: 12.641 & 33.213 & -115.584 & 1.3 \\ 2009 / 12 / 08 & 21: 16: 25.641 & 33.115 & -115.585 & 6.5 \\ 2009 / 12 / 08 & 21: 23: 47.641 & 33.196 & -115.559 & 0.2 \\ 2009 / 12 / 08 & 21: 25: 58.641 & 33.196 & -115.559 & 0.2 \\ 2009 / 12 / 08 & 21: 56: 01.641 & 33.153 & -115.638 & 10.1 \\ 2009 / 12 / 08 & 22: 10: 57.641 & 33.188 & -115.571 & 3.0 \\ 2009 / 12 / 08 & 22: 14: 16.641 & 33.196 & -115.587 & 5.3 \\ 2009 / 12 / 08 & 22: 15: 33.641 & 33.176 & -115.606 & 6.3 \\ 2009 / 12 / 08 & 22: 18: 44.641 & 33.196 & -115.559 & 0.2 \\ 2009 / 12 / 08 & 22: 19: 08.641 & 33.229 & -115.645 & 5.7 \\ 2009 / 12 / 08 & 22: 22: 17.641 & 33.196 & -115.559 & 0.2 \\ 2009 / 12 / 08 & 22: 22: 37.641 & 33.196 & -115.559 & 0.2 \\ 2009 / 12 / 08 & 22: 31: 52.641 & 33.198 & -115.571 & 0.2 \\ 2009 / 12 / 09 & 00: 25: 13.641 & 33.236 & -115.573 & 5.0 \\ 2009 / 12 / 09 & 00: 26: 03.641 & 33.201 & -115.544 & 3.4 \\ 2009 / 12 / 09 & 00: 49: 16.641 & 33.196 & -115.566 & 3.2 \\ 2009 / 12 / 09 & 00: 54: 41.641 & 33.188 & -115.571 & 3.0 \\ 2009 / 12 / 09 & 01: 00: 26.641 & 33.176 & -115.609 & 2.1 \\ 2009 / 12 / 09 & 01: 00: 52.641 & 33.229 & -115.645 & 5.7 \\ 2009 / 12 / 09 & 01: 12: 55.641 & 33.153 & -115.638 & 10.1 \\ 2009 / 12 / 09 & 01: 17: 39.641 & 33.188 & -115.571 & 3.0 \\ 2009 / 12 / 09 & 01: 34: 24.641 & 33.100 & -115.590 & 14.6 \\ 2009 / 12 / 09 & 01: 35: 41.641 & 33.196 & -115.587 & 5.3 \\ 2009 / 12 / 09 & 02: 39: 17.641 & 33.196 & -115.559 & 0.2 \\ 2009 / 12 / 09 & 03: 39: 22.641 & 33.170 & -115.600 & 1.1 \\ 2009 / 12 / 09 & 04: 49: 52.641 & 33.196 & -115.566 & 3.2 \\ 2009 / 12 / 09 & 04: 50: 17.641 & 33.212 & -115.590 & 7.2 \\ 2009 / 12 / 09 & 05: 02: 59.641 & 33.188 & -115.571 & 3.0 \\ 2009 / 12 / 09 & 05: 32: 46.641 & 33.199 & -115.571 & 3.3 \\ 2009 / 12 / 09 & 05: 51: 42.641 & 33.181 & -115.639 & 3.7 \\ 2009 / 12 / 09 & 06: 00: 37.641 & 33.179 & -115.597 & 0.1 \\ 2009 / 12 / 09 & 07: 04: 50.641 & 33.196 & -115.559 & 0.2 \\ 2009 / 12 / 09 & 10: 19: 54.641 & 33.198 & -115.571 & 0.2 \\ 2009 / 12 / 09 & 10: 51: 29.641 & 33.115 & -115.585 & 6.5 \\ 2009 / 12 / 09 & 10: 53: 49.641 & 33.196 & -115.559 & 0.2 \\ 2009 / 12 / 09 & 11: 23: 51.641 & 33.179 & -115.594 & 1.5 \\ 2009 / 12 / 09 & 11: 45: 02.641 & 33.196 & -115.559 & 0.2 \\ 2009 / 12 / 09 & 12: 01: 07.641 & 33.193 & -115.583 & 5.9\end{array}$




$\begin{array}{lllll}2009 / 12 / 09 & 12: 02: 48.641 & 33.179 & -115.594 & 1.5 \\ 2009 / 12 / 09 & 13: 19: 52.641 & 33.199 & -115.570 & 0.2 \\ 2009 / 12 / 09 & 13: 34: 57.641 & 33.212 & -115.590 & 7.2 \\ 2009 / 12 / 09 & 13: 43: 48.641 & 33.203 & -115.578 & 2.2 \\ 2009 / 12 / 09 & 13: 54: 24.641 & 33.199 & -115.570 & 0.2 \\ 2009 / 12 / 09 & 15: 38: 36.641 & 33.196 & -115.559 & 0.2 \\ 2009 / 12 / 09 & 17: 23: 49.641 & 33.203 & -115.579 & 3.5 \\ 2009 / 12 / 09 & 18: 44: 40.641 & 33.180 & -115.600 & 0.1 \\ 2009 / 12 / 09 & 18: 45: 39.641 & 33.184 & -115.609 & 2.2 \\ 2009 / 12 / 09 & 19: 09: 04.641 & 33.183 & -115.599 & 0.1 \\ 2009 / 12 / 10 & 00: 14: 29.641 & 33.196 & -115.559 & 0.2 \\ 2009 / 12 / 10 & 01: 13: 56.641 & 33.196 & -115.559 & 0.2 \\ 2009 / 12 / 10 & 02: 30: 26.641 & 33.199 & -115.570 & 0.2 \\ 2009 / 12 / 10 & 03: 13: 19.641 & 33.202 & -115.575 & 3.8 \\ 2009 / 12 / 10 & 03: 51: 37.641 & 33.201 & -115.544 & 3.4 \\ 2009 / 12 / 10 & 04: 32: 41.641 & 33.188 & -115.571 & 3.0 \\ 2009 / 12 / 10 & 04: 33: 07.641 & 33.188 & -115.571 & 3.0 \\ 2009 / 12 / 10 & 04: 33: 51.641 & 33.201 & -115.544 & 3.4 \\ 2009 / 12 / 10 & 04: 34: 10.641 & 33.115 & -115.585 & 6.5 \\ 2009 / 12 / 10 & 04: 35: 05.641 & 33.203 & -115.578 & 2.2 \\ 2009 / 12 / 10 & 04: 36: 15.641 & 33.188 & -115.571 & 3.0 \\ 2009 / 12 / 10 & 04: 38: 56.641 & 33.188 & -115.571 & 3.0 \\ 2009 / 12 / 10 & 04: 44: 55.641 & 33.179 & -115.594 & 1.5 \\ 2009 / 12 / 10 & 04: 45: 51.641 & 33.188 & -115.571 & 3.0 \\ 2009 / 12 / 10 & 04: 47: 43.641 & 33.176 & -115.606 & 6.3 \\ 2009 / 12 / 10 & 04: 52: 45.641 & 33.188 & -115.571 & 3.0 \\ 2009 / 12 / 10 & 05: 27: 29.641 & 33.213 & -115.584 & 1.3 \\ 2009 / 12 / 10 & 06: 44: 43.641 & 33.213 & -115.584 & 1.3 \\ 2009 / 12 / 10 & 06: 57: 54.641 & 33.213 & -115.584 & 1.3 \\ 2009 / 12 / 10 & 07: 26: 00.641 & 33.196 & -115.559 & 0.2 \\ 2009 / 12 / 10 & 09: 40: 54.641 & 33.115 & -115.585 & 6.5 \\ 2009 / 12 / 10 & 09: 58: 35.641 & 33.221 & -115.605 & 5.1 \\ 2009 / 12 / 10 & 12: 32: 36.641 & 33.201 & -115.544 & 3.4 \\ 2009 / 12 / 10 & 18: 17: 20.641 & 33.140 & -115.636 & 7.3 \\ 2009 / 12 / 10 & 18: 39: 59.641 & 33.199 & -115.570 & 0.2 \\ 2009 / 12 / 10 & 18: 44: 36.641 & 33.199 & -115.617 & 0.0 \\ 2009 / 12 / 10 & 19: 19: 55.641 & 33.140 & -115.636 & 7.3 \\ 2009 / 12 / 10 & 20: 36: 15.641 & 33.115 & -115.585 & 6.5 \\ 2009 / 12 / 10 & 21: 45: 59.641 & 33.199 & -115.570 & 0.2 \\ 2009 / 12 / 10 & 21: 54: 16.641 & 33.203 & -115.578 & 2.2 \\ 2009 / 12 / 10 & 22: 22: 08.641 & 33.213 & -115.584 & 1.3 \\ 2009 / 12 / 12 / 11 & 02: 01: 24.641 & 33.188 & -115.571 & 3.0 \\ 02: 02: 24.641 & 33.184 & -115.605 & 0.1 \\ 202: 03: 30.641 & 33.199 & -115.570 & 0.2 \\ 203: 04: 01.641 & 33.229 & -115.576 & 3.4 \\ 200: 45.641 & 33.212 & -115.590 & 7.2\end{array}$




$\begin{array}{lllll}2009 / 12 / 11 & 03: 27: 09.641 & 33.212 & -115.590 & 7.2 \\ 2009 / 12 / 11 & 04: 34: 32.641 & 33.214 & -115.620 & 5.3 \\ 2009 / 12 / 11 & 04: 40: 58.641 & 33.179 & -115.594 & 1.5 \\ 2009 / 12 / 11 & 09: 01: 57.641 & 33.108 & -115.602 & 8.1 \\ 2009 / 12 / 11 & 09: 02: 56.641 & 33.108 & -115.602 & 8.1 \\ 2009 / 12 / 11 & 09: 14: 31.641 & 33.108 & -115.602 & 8.1 \\ 2009 / 12 / 11 & 09: 15: 35.641 & 33.196 & -115.559 & 0.2 \\ 2009 / 12 / 11 & 09: 37: 11.641 & 33.201 & -115.544 & 3.4 \\ 2009 / 12 / 11 & 09: 49: 59.641 & 33.153 & -115.638 & 10.1 \\ 2009 / 12 / 11 & 10: 11: 46.641 & 33.196 & -115.559 & 0.2 \\ 2009 / 12 / 11 & 10: 25: 43.641 & 33.196 & -115.559 & 0.2 \\ 2009 / 12 / 11 & 10: 30: 37.641 & 33.176 & -115.609 & 3.8 \\ 2009 / 12 / 11 & 10: 32: 57.641 & 33.196 & -115.559 & 0.2 \\ 2009 / 12 / 11 & 10: 43: 42.641 & 33.196 & -115.559 & 0.2 \\ 2009 / 12 / 11 & 10: 44: 13.641 & 33.196 & -115.559 & 0.2 \\ 2009 / 12 / 11 & 10: 49: 16.641 & 33.196 & -115.559 & 0.2 \\ 2009 / 12 / 11 & 10: 50: 31.641 & 33.196 & -115.559 & 0.2 \\ 2009 / 12 / 11 & 10: 52: 42.641 & 33.196 & -115.559 & 0.2 \\ 2009 / 12 / 11 & 10: 56: 04.641 & 33.196 & -115.559 & 0.2 \\ 2009 / 12 / 11 & 10: 57: 52.641 & 33.190 & -115.574 & 3.1 \\ 2009 / 12 / 11 & 11: 00: 51.641 & 33.190 & -115.574 & 3.1 \\ 2009 / 12 / 11 & 11: 01: 13.641 & 33.196 & -115.559 & 0.2 \\ 2009 / 12 / 11 & 11: 02: 39.641 & 33.196 & -115.559 & 0.2 \\ 2009 / 12 / 11 & 11: 08: 12.641 & 33.196 & -115.559 & 0.2 \\ 2009 / 12 / 11 & 11: 10: 33.641 & 33.190 & -115.574 & 3.1 \\ 2009 / 12 / 11 & 11: 11: 24.641 & 33.190 & -115.574 & 3.1 \\ 2009 / 12 / 11 & 11: 13: 40.641 & 33.206 & -115.567 & 2.7 \\ 2009 / 12 / 11 & 11: 15: 45.641 & 33.196 & -115.559 & 0.2 \\ 2009 / 12 / 11 & 11: 23: 24.641 & 33.196 & -115.559 & 0.2 \\ 2009 / 12 / 11 & 11: 23: 36.641 & 33.182 & -115.602 & 2.5 \\ 2009 / 12 / 11 & 11: 47: 14.641 & 33.176 & -115.604 & 0.5 \\ 2009 / 12 / 11 & 12: 11: 41.641 & 33.159 & -115.641 & 0.9 \\ 2009 / 12 / 11 & 12: 14: 26.641 & 33.159 & -115.641 & 0.9 \\ 2009 / 12 / 11 & 12: 20: 00.641 & 33.159 & -115.641 & 0.9 \\ 2009 / 12 / 11 & 12: 22: 08.641 & 33.158 & -115.642 & 0.1 \\ 2009 / 12 / 11 & 12: 29: 01.641 & 33.187 & -115.601 & 1.7 \\ 2009 / 12 / 11 & 13: 02: 17.641 & 33.188 & -115.571 & 3.0 \\ 2009 / 12 / 11 & 13: 25: 50.641 & 33.181 & -115.612 & 1.1 \\ 2009 / 12 / 11 & 13: 27: 51.641 & 33.161 & -115.644 & 1.0 \\ 2009 / 12 / 11 & 13: 28: 22.641 & 33.135 & -115.644 & 6.9 \\ 2009 / 12 / 11 & 13: 32: 57.641 & 33.190 & -115.574 & 3.1 \\ 2009 / 12 / 11 & 13: 52: 36.641 & 33.206 & -115.567 & 2.7 \\ 2009 / 12 / 11 & 16: 28: 54.641 & 33.185 & -115.569 & 3.7 \\ 2009 / 12 / 11 & 17: 26: 37.641 & 33.196 & -115.559 & 0.2 \\ 2009 / 12 / 11 & 18: 45: 57.641 & 33.176 & -115.608 & 0.4 \\ 2009 / 12 / 11 & 19: 12: 12.641 & 33.229 & -115.576 & 3.4\end{array}$




$\begin{array}{lllll}2009 / 12 / 11 & 21: 55: 06.641 & 33.181 & -115.612 & 1.1 \\ 2009 / 12 / 11 & 22: 05: 05.641 & 33.189 & -115.576 & 0.1 \\ 2009 / 12 / 12 & 00: 09: 39.641 & 33.149 & -115.640 & 3.5 \\ 2009 / 12 / 12 & 00: 44: 06.641 & 33.176 & -115.620 & 1.6 \\ 2009 / 12 / 12 & 00: 45: 25.641 & 33.173 & -115.650 & 1.8 \\ 2009 / 12 / 12 & 00: 46: 17.641 & 33.170 & -115.626 & 0.6 \\ 2009 / 12 / 12 & 00: 48: 16.641 & 33.176 & -115.609 & 2.1 \\ 2009 / 12 / 12 & 00: 53: 02.641 & 33.149 & -115.640 & 3.5 \\ 2009 / 12 / 12 & 01: 12: 09.641 & 33.196 & -115.559 & 0.2 \\ 2009 / 12 / 12 & 02: 58: 03.641 & 33.173 & -115.650 & 1.8 \\ 2009 / 12 / 12 & 06: 48: 01.641 & 33.182 & -115.602 & 2.5 \\ 2009 / 12 / 12 & 07: 34: 28.641 & 33.179 & -115.614 & 1.8 \\ 2009 / 12 / 12 & 08: 56: 39.641 & 33.108 & -115.602 & 8.1 \\ 2009 / 12 / 12 & 10: 32: 12.641 & 33.142 & -115.628 & 10.3 \\ 2009 / 12 / 12 & 10: 44: 37.641 & 33.108 & -115.602 & 8.1 \\ 2009 / 12 / 12 & 10: 57: 22.641 & 33.161 & -115.651 & 0.7 \\ 2009 / 12 / 12 & 11: 38: 53.641 & 33.142 & -115.628 & 10.3 \\ 2009 / 12 / 12 & 11: 42: 49.641 & 33.108 & -115.602 & 8.1 \\ 2009 / 12 / 12 & 11: 55: 05.641 & 33.142 & -115.628 & 10.3 \\ 2009 / 12 / 12 & 17: 10: 36.641 & 33.100 & -115.590 & 14.6 \\ 2009 / 12 / 12 & 17: 12: 42.641 & 33.192 & -115.572 & 1.6 \\ 2009 / 12 / 12 & 17: 13: 18.641 & 33.161 & -115.651 & 0.7 \\ 2009 / 12 / 12 & 17: 20: 08.641 & 33.197 & -115.644 & 3.0 \\ 2009 / 12 / 12 & 17: 44: 43.641 & 33.251 & -115.564 & 0.1 \\ 2009 / 12 / 12 & 17: 44: 57.641 & 33.251 & -115.564 & 0.1 \\ 2009 / 12 / 12 & 18: 04: 03.641 & 33.161 & -115.651 & 0.7 \\ 2009 / 12 / 12 & 18: 04: 32.641 & 33.161 & -115.651 & 0.7 \\ 2009 / 12 / 12 & 18: 07: 04.641 & 33.179 & -115.597 & 0.1 \\ 2009 / 12 / 12 & 18: 08: 45.641 & 33.182 & -115.602 & 2.5 \\ 2009 / 12 / 12 & 18: 22: 14.641 & 33.161 & -115.651 & 0.7 \\ 2009 / 12 / 12 & 20: 52: 42.641 & 33.176 & -115.604 & 0.5 \\ 2009 / 12 / 13 & 00: 14: 10.641 & 33.192 & -115.626 & 7.7 \\ 2009 / 12 / 13 & 02: 22: 54.641 & 33.158 & -115.638 & 0.4 \\ 2009 / 12 / 13 & 04: 27: 59.641 & 33.117 & -115.613 & 7.9 \\ 2009 / 12 / 13 & 14: 16: 36.641 & 33.172 & -115.622 & 4.0 \\ 2009 / 12 / 13 & 19: 47: 19.641 & 33.153 & -115.638 & 10.1 \\ 2009 / 12 / 14 & 15: 40: 59.641 & 33.176 & -115.609 & 2.1 \\ 2009 / 12 / 14 & 16: 25: 31.641 & 33.135 & -115.644 & 6.9 \\ 2009 / 12 / 14 & 19: 21: 19.641 & 33.213 & -115.584 & 1.3 \\ 2009 / 12 / 14 & 23: 57: 58.641 & 33.183 & -115.599 & 0.1 \\ 2009 / 12 / 15 & 01: 43: 26.641 & 33.108 & -115.602 & 8.1 \\ 2009 / 12 / 15 & 04: 08: 53.641 & 33.176 & -115.608 & 0.4 \\ 2009 / 12 / 15 & 07: 31: 53.641 & 33.173 & -115.610 & 1.3 \\ 2009 / 12 / 15 & 08: 57: 36.641 & 33.184 & -115.605 & 0.1 \\ 2009 / 12 / 15 & 17: 43: 48.641 & 33.135 & -115.644 & 6.9 \\ 2009 / 12 / 15 & 17: 44: 21.641 & 33.158 & -115.638 & 0.4\end{array}$




$\begin{array}{lllll}2009 / 12 / 15 & 17: 46: 20.641 & 33.108 & -115.602 & 8.1 \\ 2009 / 12 / 15 & 18: 23: 26.641 & 33.108 & -115.602 & 8.1 \\ 2009 / 12 / 15 & 19: 37: 27.641 & 33.208 & -115.582 & 2.5 \\ 2009 / 12 / 15 & 19: 41: 23.641 & 33.193 & -115.632 & 3.5 \\ 2009 / 12 / 15 & 19: 41: 48.641 & 33.183 & -115.599 & 0.1 \\ 2009 / 12 / 15 & 19: 42: 33.641 & 33.197 & -115.596 & 3.0 \\ 2009 / 12 / 15 & 20: 35: 46.641 & 33.178 & -115.611 & 1.6 \\ 2009 / 12 / 15 & 22: 11: 23.641 & 33.176 & -115.608 & 0.4 \\ 2009 / 12 / 15 & 22: 13: 54.641 & 33.170 & -115.600 & 1.1 \\ 2009 / 12 / 15 & 22: 37: 14.641 & 33.117 & -115.613 & 7.9 \\ 2009 / 12 / 15 & 23: 37: 31.641 & 33.189 & -115.567 & 2.5 \\ 2009 / 12 / 16 & 01: 38: 46.641 & 33.182 & -115.602 & 2.5 \\ 2009 / 12 / 16 & 03: 07: 34.641 & 33.142 & -115.628 & 10.3 \\ 2009 / 12 / 16 & 21: 24: 33.641 & 33.183 & -115.599 & 0.1 \\ 2009 / 12 / 17 & 00: 07: 59.641 & 33.181 & -115.665 & 4.2 \\ 2009 / 12 / 17 & 02: 14: 58.641 & 33.142 & -115.628 & 10.3 \\ 2009 / 12 / 17 & 08: 24: 38.641 & 33.190 & -115.612 & 1.0 \\ 2009 / 12 / 17 & 12: 50: 45.641 & 33.231 & -115.605 & 4.8 \\ 2009 / 12 / 17 & 12: 51: 20.641 & 33.187 & -115.601 & 1.7 \\ 2009 / 12 / 17 & 14: 14: 31.641 & 33.181 & -115.665 & 4.2 \\ 2009 / 12 / 17 & 14: 17: 41.641 & 33.181 & -115.665 & 4.2 \\ 2009 / 12 / 17 & 14: 18: 12.641 & 33.214 & -115.620 & 5.3 \\ 2009 / 12 / 17 & 14: 24: 37.641 & 33.181 & -115.665 & 4.2 \\ 2009 / 12 / 17 & 15: 02: 42.641 & 33.159 & -115.641 & 0.9 \\ 2009 / 12 / 17 & 16: 57: 42.641 & 33.188 & -115.573 & 0.2 \\ 2009 / 12 / 17 & 17: 54: 50.641 & 33.183 & -115.599 & 0.1 \\ 2009 / 12 / 17 & 18: 16: 59.641 & 33.183 & -115.599 & 0.1 \\ 2009 / 12 / 17 & 21: 39: 43.641 & 33.100 & -115.590 & 14.6 \\ 2009 / 12 / 18 & 10: 50: 54.641 & 33.179 & -115.621 & 4.8 \\ 2009 / 12 / 18 & 22: 56: 40.641 & 33.229 & -115.576 & 3.4 \\ 2009 / 12 / 18 & 22: 56: 56.641 & 33.199 & -115.571 & 3.3 \\ 2009 / 12 / 19 & 08: 12: 21.641 & 33.186 & -115.612 & 0.6 \\ 2009 / 12 / 19 & 14: 22: 55.641 & 33.183 & -115.677 & 0.5 \\ 2009 / 12 / 19 & 14: 23: 42.641 & 33.158 & -115.642 & 0.1 \\ 2009 / 12 / 19 & 19: 02: 42.641 & 33.183 & -115.599 & 0.1 \\ 2009 / 12 / 19 & 23: 24: 12.641 & 33.184 & -115.609 & 2.2 \\ 2009 / 12 / 19 & 23: 57: 48.641 & 33.188 & -115.571 & 3.0 \\ 2009 / 12 / 20 & 00: 49: 37.641 & 33.188 & -115.571 & 3.0 \\ 2009 / 12 / 20 & 02: 06: 39.641 & 33.188 & -115.573 & 1.2 \\ 2009 / 12 / 20 & 02: 36: 25.641 & 33.189 & -115.576 & 0.1 \\ 2009 / 12 / 20 & 09: 45: 41.641 & 33.158 & -115.642 & 0.1 \\ 2009 / 12 / 20 & 09: 48: 12.641 & 33.178 & -115.626 & 1.3 \\ 2009 / 12 / 20 & 10: 34: 01.641 & 33.181 & -115.601 & 0.7 \\ 2009 / 12 / 20 & 10: 55: 47.641 & 33.189 & -115.576 & 0.1 \\ 2009 / 12 / 20 & 15: 39: 24.641 & 33.201 & -115.544 & 3.4 \\ 2009 / 12 / 20 & 17: 41: 16.641 & 33.201 & -115.544 & 3.4\end{array}$




$\begin{array}{lllll}2009 / 12 / 21 & 04: 33: 52.641 & 33.187 & -115.601 & 1.7 \\ 2009 / 12 / 21 & 11: 40: 46.641 & 33.248 & -115.633 & 2.9 \\ 2009 / 12 / 21 & 13: 33: 42.641 & 33.149 & -115.640 & 3.5 \\ 2009 / 12 / 21 & 13: 56: 33.641 & 33.108 & -115.602 & 8.1 \\ 2009 / 12 / 21 & 23: 32: 45.641 & 33.182 & -115.602 & 2.5 \\ 2009 / 12 / 22 & 07: 59: 44.641 & 33.182 & -115.602 & 2.5 \\ 2009 / 12 / 22 & 12: 18: 08.641 & 33.181 & -115.665 & 4.2 \\ 2009 / 12 / 22 & 12: 50: 36.641 & 33.192 & -115.572 & 1.6 \\ 2009 / 12 / 22 & 14: 37: 51.641 & 33.176 & -115.609 & 2.1 \\ 2009 / 12 / 22 & 22: 33: 40.641 & 33.176 & -115.609 & 2.1 \\ 2009 / 12 / 22 & 22: 34: 16.641 & 33.173 & -115.610 & 1.3 \\ 2009 / 12 / 22 & 22: 34: 39.641 & 33.176 & -115.608 & 0.4 \\ 2009 / 12 / 22 & 22: 39: 08.641 & 33.181 & -115.665 & 4.2 \\ 2009 / 12 / 23 & 02: 37: 33.641 & 33.176 & -115.609 & 2.1 \\ 2009 / 12 / 23 & 02: 37: 33.641 & 33.136 & -115.649 & 5.3 \\ 2009 / 12 / 23 & 06: 14: 06.641 & 33.181 & -115.665 & 4.2 \\ 2009 / 12 / 23 & 16: 22: 59.641 & 33.183 & -115.599 & 0.1 \\ 2009 / 12 / 23 & 17: 49: 54.641 & 33.183 & -115.606 & 0.1 \\ 2009 / 12 / 23 & 20: 36: 31.641 & 33.183 & -115.599 & 0.1 \\ 2009 / 12 / 23 & 20: 36: 58.641 & 33.183 & -115.599 & 0.1 \\ 2009 / 12 / 23 & 20: 39: 38.641 & 33.176 & -115.608 & 0.4 \\ 2009 / 12 / 23 & 21: 58: 41.641 & 33.177 & -115.606 & 0.1 \\ 2009 / 12 / 24 & 03: 49: 04.641 & 33.228 & -115.626 & 3.7 \\ 2009 / 12 / 24 & 06: 17: 49.641 & 33.185 & -115.569 & 3.7 \\ 2009 / 12 / 24 & 06: 18: 23.641 & 33.187 & -115.601 & 1.7 \\ 2009 / 12 / 24 & 06: 18: 39.641 & 33.179 & -115.614 & 1.8 \\ 2009 / 12 / 24 & 06: 19: 03.641 & 33.187 & -115.601 & 1.7 \\ 2009 / 12 / 24 & 06: 22: 46.641 & 33.177 & -115.606 & 0.1 \\ 2009 / 12 / 24 & 06: 25: 14.641 & 33.190 & -115.612 & 1.0 \\ 2009 / 12 / 24 & 07: 22: 35.641 & 33.117 & -115.613 & 7.9 \\ 2009 / 12 / 24 & 08: 28: 16.641 & 33.179 & -115.621 & 4.8 \\ 2009 / 12 / 24 & 08: 38: 31.641 & 33.183 & -115.599 & 0.1 \\ 2009 / 12 / 24 & 08: 40: 56.641 & 33.208 & -115.582 & 2.5 \\ 2009 / 12 / 24 & 16: 01: 06.641 & 33.192 & -115.572 & 1.6 \\ 2009 / 12 / 24 & 23: 31: 28.641 & 33.184 & -115.608 & 0.3 \\ 2009 / 12 / 24 & 23: 57: 39.641 & 33.173 & -115.610 & 1.3 \\ 2009 / 12 / 25 & 02: 32: 27.641 & 33.179 & -115.594 & 1.5 \\ 2009 / 12 / 25 & 02: 32: 50.641 & 33.179 & -115.594 & 1.5 \\ 2009 / 12 / 25 & 07: 13: 57.641 & 33.229 & -115.576 & 3.4 \\ 2009 / 12 / 25 & 22: 16: 16.641 & 33.108 & -115.602 & 8.1 \\ 2009 / 12 / 25 & 22: 18: 47.641 & 33.108 & -115.602 & 8.1 \\ 2009 / 12 / 26 & 02: 36: 47.641 & 33.228 & -115.626 & 3.7 \\ 2009 / 12 / 26 & 10: 47: 13.641 & 33.179 & -115.614 & 1.8 \\ 2009 / 12 / 26 & 14: 58: 01.641 & 33.183 & -115.606 & 0.1\end{array}$




$\begin{array}{lllll}2009 / 12 / 26 & 14: 59: 12.641 & 33.183 & -115.606 & 0.1 \\ 2009 / 12 / 26 & 14: 59: 23.641 & 33.183 & -115.606 & 0.1 \\ 2009 / 12 / 26 & 15: 00: 51.641 & 33.183 & -115.606 & 0.1 \\ 2009 / 12 / 26 & 15: 01: 41.641 & 33.183 & -115.606 & 0.1 \\ 2009 / 12 / 26 & 15: 03: 47.641 & 33.183 & -115.606 & 0.1 \\ 2009 / 12 / 26 & 15: 06: 47.641 & 33.181 & -115.611 & 0.4 \\ 2009 / 12 / 26 & 15: 07: 44.641 & 33.179 & -115.614 & 1.8 \\ 2009 / 12 / 26 & 15: 09: 34.641 & 33.190 & -115.604 & 1.2 \\ 2009 / 12 / 26 & 19: 50: 32.641 & 33.149 & -115.640 & 3.5 \\ 2009 / 12 / 26 & 22: 55: 06.641 & 33.229 & -115.576 & 3.4 \\ 2009 / 12 / 27 & 09: 53: 47.641 & 33.209 & -115.590 & 3.1 \\ 2009 / 12 / 27 & 09: 55: 10.641 & 33.226 & -115.588 & 4.6 \\ 2009 / 12 / 27 & 19: 57: 43.641 & 33.179 & -115.614 & 1.8 \\ 2009 / 12 / 27 & 20: 10: 14.641 & 33.197 & -115.659 & 6.4 \\ 2009 / 12 / 28 & 11: 30: 03.641 & 33.149 & -115.640 & 3.5 \\ 2009 / 12 / 28 & 16: 29: 40.641 & 33.181 & -115.610 & 0.9 \\ 2009 / 12 / 28 & 16: 41: 03.641 & 33.183 & -115.599 & 0.1 \\ 2009 / 12 / 28 & 18: 55: 33.641 & 33.170 & -115.600 & 1.1 \\ 2009 / 12 / 28 & 19: 28: 34.641 & 33.108 & -115.602 & 8.1 \\ 2009 / 12 / 28 & 23: 25: 18.641 & 33.201 & -115.544 & 3.4 \\ 2009 / 12 / 29 & 10: 21: 56.641 & 33.192 & -115.567 & 3.1 \\ 2009 / 12 / 30 & 11: 36: 31.641 & 33.198 & -115.571 & 0.2 \\ 2009 / 12 / 30 & 11: 37: 15.641 & 33.198 & -115.571 & 0.2 \\ 2009 / 12 / 30 & 11: 37: 52.641 & 33.182 & -115.602 & 2.5 \\ 2009 / 12 / 30 & 17: 51: 56.641 & 33.140 & -115.636 & 7.3 \\ 2009 / 12 / 30 & 18: 12: 14.641 & 33.140 & -115.636 & 7.3 \\ 2009 / 12 / 30 & 18: 50: 26.641 & 33.108 & -115.602 & 8.1 \\ 2009 / 12 / 30 & 18: 50: 45.641 & 33.201 & -115.544 & 3.4 \\ 2009 / 12 / 30 & 18: 52: 32.641 & 33.181 & -115.659 & 6.0 \\ 2009 / 12 / 30 & 18: 53: 02.641 & 33.179 & -115.614 & 1.8 \\ 2009 / 12 / 30 & 20: 23: 55.641 & 33.149 & -115.640 & 3.5 \\ 2009 / 12 / 30 & 20: 28: 36.641 & 33.183 & -115.606 & 0.1 \\ 2009 / 12 / 30 & 20: 35: 16.641 & 33.194 & -115.583 & 3.0 \\ 2009 / 12 / 30 & 23: 33: 05.641 & 33.161 & -115.651 & 0.7 \\ 2009 / 12 / 31 & 04: 10: 09.641 & 33.184 & -115.597 & 0.1 \\ 2009 / 12 / 31 & 06: 15: 22.641 & 33.161 & -115.644 & 0.3 \\ 2009 / 12 / 31 & 06: 23: 57.641 & 33.179 & -115.614 & 1.8 \\ 2009 / 12 / 31 & 06: 31: 41.641 & 33.179 & -115.614 & 1.8 \\ 2009 / 12 / 31 & 08: 59: 17.641 & 33.186 & -115.593 & 0.1 \\ 2009 / 12 / 31 & 09: 03: 21.641 & 33.186 & -115.593 & 0.1 \\ 2009 / 12 / 31 & 09: 10: 25.641 & 33.186 & -115.593 & 0.1 \\ 2009 / 12 / 31 & 09: 27: 46.641 & 33.186 & -115.593 & 0.1 \\ 2009 / 12 / 31 & 09: 37: 20.641 & 33.186 & -115.593 & 0.1 \\ 209 / 31 & 10: 23: 57.641 & 33.186 & -115.593 & 0.1 \\ 215: 03: 10.641 & 33.193 & -115.615 & 3.9\end{array}$




$\begin{array}{lllll}2009 / 12 / 31 & 17: 19: 33.641 & 33.184 & -115.608 & 0.3 \\ 2009 / 12 / 31 & 18: 19: 59.641 & 33.181 & -115.665 & 4.2 \\ 2009 / 12 / 31 & 18: 20: 47.641 & 33.229 & -115.576 & 3.4 \\ 2009 / 12 / 31 & 21: 17: 38.641 & 33.218 & -115.639 & 3.0 \\ 2009 / 12 / 31 & 21: 48: 27.641 & 33.188 & -115.571 & 3.0 \\ 2010 / 01 / 01 & 01: 16: 53.639 & 33.193 & -115.583 & 5.9 \\ 2010 / 01 / 01 & 03: 43: 42.639 & 33.186 & -115.612 & 0.6 \\ 2010 / 01 / 01 & 04: 23: 04.639 & 33.182 & -115.603 & 0.1 \\ 2010 / 01 / 01 & 15: 53: 31.639 & 33.208 & -115.631 & 5.0 \\ 2010 / 01 / 01 & 23: 27: 31.639 & 33.158 & -115.642 & 0.1 \\ 2010 / 01 / 02 & 00: 10: 06.639 & 33.231 & -115.655 & 4.8 \\ 2010 / 01 / 02 & 05: 45: 23.639 & 33.184 & -115.608 & 0.3 \\ 2010 / 01 / 02 & 23: 02: 47.639 & 33.100 & -115.590 & 14.6 \\ 2010 / 01 / 02 & 23: 05: 31.639 & 33.100 & -115.590 & 14.6 \\ 2010 / 01 / 02 & 23: 12: 54.639 & 33.180 & -115.603 & 0.1 \\ 2010 / 01 / 03 & 03: 38: 08.639 & 33.190 & -115.608 & 2.2 \\ 2010 / 01 / 03 & 03: 39: 00.639 & 33.190 & -115.608 & 2.2 \\ 2010 / 01 / 03 & 17: 12: 36.639 & 33.188 & -115.600 & 0.1 \\ 2010 / 01 / 03 & 19: 42: 08.639 & 33.130 & -115.634 & 0.0 \\ 2010 / 01 / 04 & 07: 55: 31.639 & 33.183 & -115.599 & 0.1 \\ 2010 / 01 / 04 & 08: 21: 34.639 & 33.115 & -115.585 & 6.5 \\ 2010 / 01 / 04 & 10: 55: 26.639 & 33.184 & -115.601 & 3.5 \\ 2010 / 01 / 04 & 10: 56: 56.639 & 33.190 & -115.604 & 1.2 \\ 2010 / 01 / 04 & 11: 47: 07.639 & 33.183 & -115.599 & 0.1 \\ 2010 / 01 / 04 & 11: 51: 11.639 & 33.183 & -115.599 & 0.1 \\ 2010 / 01 / 04 & 12: 01: 53.639 & 33.140 & -115.636 & 7.3 \\ 2010 / 01 / 04 & 13: 11: 41.639 & 33.183 & -115.599 & 0.1 \\ 2010 / 01 / 04 & 13: 12: 03.639 & 33.183 & -115.599 & 0.1 \\ 2010 / 01 / 04 & 16: 51: 08.639 & 33.108 & -115.602 & 8.1 \\ 2010 / 01 / 04 & 23: 35: 37.639 & 33.218 & -115.639 & 3.0 \\ 2010 / 01 / 05 & 05: 52: 29.639 & 33.188 & -115.600 & 0.1 \\ 2010 / 01 / 05 & 05: 55: 02.639 & 33.181 & -115.659 & 6.0 \\ 2010 / 01 / 05 & 08: 11: 55.639 & 33.176 & -115.606 & 6.3 \\ 2010 / 01 / 05 & 08: 33: 13.639 & 33.203 & -115.578 & 2.2 \\ 2010 / 01 / 05 & 09: 34: 12.639 & 33.180 & -115.609 & 0.0 \\ 2010 / 01 / 05 & 11: 51: 10.639 & 33.184 & -115.611 & 2.7 \\ 2010 / 01 / 05 & 11: 52: 44.639 & 33.179 & -115.614 & 1.8 \\ 2010 / 01 / 05 & 12: 19: 42.639 & 33.135 & -115.644 & 6.9 \\ 2010 / 01 / 05 & 16: 14: 38.639 & 33.212 & -115.590 & 7.2 \\ 2010 / 01 / 05 & 23: 14: 14.639 & 33.179 & -115.614 & 1.8 \\ 2010 / 01 / 06 & 02: 47: 46.639 & 33.192 & -115.579 & 4.3 \\ 2010 / 01 / 06 & 03: 44: 47.639 & 33.136 & -115.649 & 5.3 \\ 2010 / 01 / 06 & 04: 40: 13.639 & 33.176 & -115.604 & 0.0 \\ 2010 / 01 / 06 & 10: 11: 08.639 & 33.100 & -115.590 & 14.6 \\ 2010 / 01 / 06 & 10: 12: 13.639 & 33.190 & -115.608 & 2.2 \\ 2010 / 01 / 06 & 11: 24: 10.639 & 33.130 & -115.634 & 0.0\end{array}$




$\begin{array}{lllll}2010 / 01 / 06 & 11: 55: 24.639 & 33.181 & -115.608 & 1.0 \\ 2010 / 01 / 06 & 14: 25: 46.639 & 33.186 & -115.612 & 0.6 \\ 2010 / 01 / 06 & 18: 53: 41.639 & 33.160 & -115.633 & 4.6 \\ 2010 / 01 / 06 & 22: 31: 58.639 & 33.188 & -115.613 & 2.5 \\ 2010 / 01 / 06 & 22: 42: 30.639 & 33.157 & -115.641 & 2.2 \\ 2010 / 01 / 07 & 03: 33: 12.639 & 33.231 & -115.655 & 4.8 \\ 2010 / 01 / 07 & 05: 05: 05.639 & 33.203 & -115.578 & 2.2 \\ 2010 / 01 / 07 & 05: 29: 50.639 & 33.179 & -115.594 & 1.5 \\ 2010 / 01 / 07 & 14: 31: 02.639 & 33.194 & -115.583 & 3.0 \\ 2010 / 01 / 07 & 16: 04: 29.639 & 33.201 & -115.544 & 3.4 \\ 2010 / 01 / 09 & 09: 23: 16.639 & 33.149 & -115.640 & 3.5 \\ 2010 / 01 / 09 & 11: 33: 58.639 & 33.183 & -115.607 & 0.0 \\ 2010 / 01 / 09 & 12: 03: 04.639 & 33.118 & -115.610 & 9.1 \\ 2010 / 01 / 09 & 12: 58: 56.639 & 33.176 & -115.604 & 0.5 \\ 2010 / 01 / 09 & 12: 59: 20.639 & 33.170 & -115.600 & 1.1 \\ 2010 / 01 / 09 & 12: 59: 41.639 & 33.176 & -115.608 & 0.4 \\ 2010 / 01 / 09 & 13: 13: 56.639 & 33.176 & -115.608 & 0.4 \\ 2010 / 01 / 09 & 13: 14: 24.639 & 33.188 & -115.599 & 0.8 \\ 2010 / 01 / 09 & 13: 26: 58.639 & 33.181 & -115.603 & 1.4 \\ 2010 / 01 / 09 & 13: 27: 16.639 & 33.187 & -115.622 & 4.0 \\ 2010 / 01 / 09 & 14: 36: 07.639 & 33.208 & -115.582 & 2.5 \\ 2010 / 01 / 09 & 15: 33: 21.639 & 33.174 & -115.603 & 0.1 \\ 2010 / 01 / 09 & 16: 00: 35.639 & 33.181 & -115.603 & 1.4 \\ 2010 / 01 / 09 & 16: 01: 41.639 & 33.170 & -115.600 & 1.1 \\ 2010 / 01 / 09 & 16: 28: 04.639 & 33.176 & -115.618 & 3.7 \\ 2010 / 01 / 09 & 16: 49: 42.639 & 33.193 & -115.615 & 3.9 \\ 2010 / 01 / 09 & 17: 29: 50.639 & 33.198 & -115.593 & 2.3 \\ 2010 / 01 / 09 & 18: 54: 36.639 & 33.176 & -115.609 & 2.1 \\ 2010 / 01 / 09 & 19: 10: 07.639 & 33.176 & -115.608 & 0.4 \\ 2010 / 01 / 09 & 19: 55: 54.639 & 33.174 & -115.603 & 0.1 \\ 2010 / 01 / 09 & 20: 33: 07.639 & 33.176 & -115.604 & 0.5 \\ 2010 / 01 / 09 & 22: 46: 27.639 & 33.176 & -115.608 & 0.4 \\ 2010 / 01 / 09 & 23: 47: 30.639 & 33.176 & -115.608 & 0.4 \\ 2010 / 01 / 10 & 04: 31: 33.639 & 33.118 & -115.610 & 9.1 \\ 2010 / 01 / 10 & 06: 43: 40.639 & 33.179 & -115.597 & 0.1 \\ 2010 / 01 / 10 & 08: 56: 56.639 & 33.182 & -115.602 & 2.5 \\ 2010 / 01 / 10 & 11: 49: 57.639 & 33.158 & -115.642 & 0.1 \\ 2010 / 01 / 10 & 12: 21: 28.639 & 33.198 & -115.593 & 2.3 \\ 2010 / 01 / 10 & 12: 27: 23.639 & 33.180 & -115.603 & 0.1 \\ 20101 / 01 / 10 & 12: 50: 22.639 & 33.176 & -115.604 & 0.5 \\ 201 / 10 & 13: 56: 10.639 & 33.175 & -115.600 & 1.1 \\ 20: 38.639 & 33.179 & -115.621 & 4.8 \\ 20 & 13: 22: 30.639 & 33.179 & -115.594 & 1.5 \\ 2010 & 13: 24: 01.639 & 33.190 & -115.604 & 1.2 \\ 203: 01.639 & 33.175 & -115.600 & 1.1 \\ 204: 19.639 & 33.190 & -115.608 & 2.2\end{array}$




$\begin{array}{lllll}2010 / 01 / 10 & 15: 14: 39.639 & 33.179 & -115.594 & 1.5 \\ 2010 / 01 / 10 & 15: 29: 00.639 & 33.190 & -115.608 & 2.2 \\ 2010 / 01 / 10 & 15: 33: 46.639 & 33.190 & -115.608 & 2.2 \\ 2010 / 01 / 10 & 15: 37: 22.639 & 33.190 & -115.608 & 2.2 \\ 2010 / 01 / 10 & 16: 44: 26.639 & 33.117 & -115.613 & 7.9 \\ 2010 / 01 / 10 & 16: 47: 11.639 & 33.181 & -115.603 & 1.4 \\ 2010 / 01 / 10 & 17: 29: 29.639 & 33.176 & -115.609 & 2.1 \\ 2010 / 01 / 10 & 18: 38: 37.639 & 33.182 & -115.602 & 2.5 \\ 2010 / 01 / 10 & 18: 45: 49.639 & 33.181 & -115.611 & 0.4 \\ 2010 / 01 / 10 & 19: 20: 08.639 & 33.172 & -115.622 & 4.0 \\ 2010 / 01 / 10 & 19: 34: 38.639 & 33.190 & -115.608 & 2.2 \\ 2010 / 01 / 10 & 19: 38: 44.639 & 33.183 & -115.599 & 0.1 \\ 2010 / 01 / 10 & 20: 15: 49.639 & 33.168 & -115.609 & 0.7 \\ 2010 / 01 / 10 & 20: 46: 22.639 & 33.175 & -115.600 & 1.1 \\ 2010 / 01 / 10 & 22: 31: 40.639 & 33.175 & -115.600 & 1.1 \\ 2010 / 01 / 10 & 22: 44: 49.639 & 33.188 & -115.599 & 0.8 \\ 2010 / 01 / 10 & 22: 50: 31.639 & 33.175 & -115.603 & 0.1 \\ 2010 / 01 / 10 & 22: 51: 08.639 & 33.181 & -115.603 & 1.4 \\ 2010 / 01 / 10 & 23: 31: 43.639 & 33.175 & -115.610 & 2.4 \\ 2010 / 01 / 10 & 23: 32: 37.639 & 33.175 & -115.600 & 1.1 \\ 2010 / 01 / 10 & 23: 46: 54.639 & 33.179 & -115.597 & 0.1 \\ 2010 / 01 / 10 & 23: 47: 44.639 & 33.179 & -115.597 & 0.1 \\ 2010 / 01 / 10 & 23: 48: 11.639 & 33.179 & -115.597 & 0.1 \\ 2010 / 01 / 10 & 23: 49: 02.639 & 33.176 & -115.604 & 0.0 \\ 2010 / 01 / 10 & 23: 49: 27.639 & 33.193 & -115.583 & 5.9 \\ 2010 / 01 / 10 & 23: 50: 55.639 & 33.175 & -115.600 & 1.1 \\ 2010 / 01 / 10 & 23: 53: 21.639 & 33.179 & -115.597 & 0.1 \\ 2010 / 01 / 10 & 23: 55: 34.639 & 33.179 & -115.597 & 0.1 \\ 2010 / 01 / 11 & 00: 07: 13.639 & 33.176 & -115.604 & 0.0 \\ 2010 / 01 / 11 & 00: 08: 33.639 & 33.192 & -115.567 & 3.1 \\ 2010 / 01 / 11 & 00: 25: 35.639 & 33.179 & -115.597 & 0.1 \\ 2010 / 01 / 11 & 00: 30: 30.639 & 33.180 & -115.603 & 0.1 \\ 2010 / 01 / 11 & 01: 05: 11.639 & 33.183 & -115.599 & 0.1 \\ 2010 / 01 / 11 & 01: 05: 41.639 & 33.190 & -115.608 & 2.2 \\ 2010 / 01 / 11 & 01: 12: 05.639 & 33.229 & -115.576 & 3.4 \\ 2010 / 01 / 11 & 01: 20: 55.639 & 33.179 & -115.597 & 0.1 \\ 2010 / 01 / 11 & 02: 10: 14.639 & 33.176 & -115.618 & 3.7 \\ 2010 / 01 / 11 & 03: 07: 16.639 & 33.177 & -115.614 & 1.2 \\ 2010 / 01 / 11 & 03: 24: 23.639 & 33.177 & -115.606 & 0.1 \\ 2010 / 01 / 11 & 04: 13: 10.639 & 33.185 & -115.569 & 3.7 \\ 2010 / 01 / 11 & 04: 52: 38.639 & 33.174 & -115.603 & 0.1 \\ 2010 / 01 / 11 & 05: 45: 39.639 & 33.100 & -115.590 & 14.6 \\ 2010 / 01 / 11 & 05: 46: 50.639 & 33.176 & -115.608 & 0.4 \\ 2010 / 01 / 11 & 05: 47: 19.639 & 33.159 & -115.641 & 0.9 \\ 2010 / 01 / 11 & 05: 47: 47.639 & 33.170 & -115.626 & 0.6 \\ 2010 / 01 / 11 & 05: 49: 45.639 & 33.182 & -115.602 & 2.5\end{array}$




$\begin{array}{lllll}2010 / 01 / 11 & 05: 49: 58.639 & 33.176 & -115.608 & 0.4 \\ 2010 / 01 / 11 & 06: 06: 45.639 & 33.180 & -115.603 & 0.1 \\ 2010 / 01 / 11 & 06: 07: 00.639 & 33.179 & -115.614 & 1.8 \\ 2010 / 01 / 11 & 06: 43: 28.639 & 33.176 & -115.604 & 0.0 \\ 2010 / 01 / 11 & 07: 19: 33.639 & 33.159 & -115.641 & 0.9 \\ 2010 / 01 / 11 & 07: 22: 49.639 & 33.175 & -115.601 & 0.1 \\ 2010 / 01 / 11 & 07: 29: 32.639 & 33.108 & -115.602 & 8.1 \\ 2010 / 01 / 11 & 07: 37: 19.639 & 33.179 & -115.594 & 1.5 \\ 2010 / 01 / 11 & 07: 37: 46.639 & 33.177 & -115.614 & 1.2 \\ 2010 / 01 / 11 & 07: 38: 54.639 & 33.170 & -115.600 & 1.1 \\ 2010 / 01 / 11 & 07: 39: 30.639 & 33.181 & -115.611 & 0.4 \\ 2010 / 01 / 11 & 08: 55: 31.639 & 33.143 & -115.638 & 4.8 \\ 2010 / 01 / 11 & 12: 03: 35.639 & 33.179 & -115.597 & 0.1 \\ 2010 / 01 / 11 & 12: 57: 12.639 & 33.181 & -115.608 & 1.0 \\ 2010 / 01 / 11 & 12: 57: 41.639 & 33.176 & -115.608 & 0.4 \\ 2010 / 01 / 11 & 15: 54: 19.639 & 33.174 & -115.603 & 0.1 \\ 2010 / 01 / 11 & 16: 01: 05.639 & 33.176 & -115.609 & 2.1 \\ 2010 / 01 / 11 & 16: 52: 25.639 & 33.176 & -115.604 & 0.5 \\ 2010 / 01 / 11 & 16: 54: 10.639 & 33.179 & -115.594 & 1.5 \\ 2010 / 01 / 11 & 16: 54: 51.639 & 33.179 & -115.594 & 1.5 \\ 2010 / 01 / 11 & 19: 03: 57.639 & 33.181 & -115.665 & 4.2 \\ 2010 / 01 / 11 & 19: 38: 30.639 & 33.130 & -115.634 & 0.0 \\ 2010 / 01 / 11 & 20: 40: 33.639 & 33.159 & -115.641 & 0.9 \\ 2010 / 01 / 11 & 21: 26: 36.639 & 33.174 & -115.603 & 0.1 \\ 2010 / 01 / 11 & 21: 26: 48.639 & 33.174 & -115.603 & 0.1 \\ 2010 / 01 / 11 & 22: 17: 10.639 & 33.179 & -115.597 & 0.1 \\ 2010 / 01 / 11 & 22: 19: 51.639 & 33.179 & -115.597 & 0.1 \\ 2010 / 01 / 11 & 22: 30: 23.639 & 33.175 & -115.600 & 1.1 \\ 2010 / 01 / 12 & 00: 59: 47.639 & 33.177 & -115.635 & 5.3 \\ 2010 / 01 / 12 & 01: 02: 21.639 & 33.179 & -115.614 & 1.8 \\ 2010 / 01 / 12 & 01: 40: 15.639 & 33.176 & -115.604 & 0.5 \\ 2010 / 01 / 12 & 01: 40: 50.639 & 33.181 & -115.611 & 0.4 \\ 2010 / 01 / 12 & 01: 42: 25.639 & 33.229 & -115.576 & 3.4 \\ 2010 / 01 / 12 & 01: 51: 33.639 & 33.175 & -115.603 & 0.1 \\ 2010 / 01 / 12 & 02: 07: 20.639 & 33.170 & -115.600 & 1.1 \\ 2010 / 01 / 12 & 02: 15: 04.639 & 33.175 & -115.603 & 0.1 \\ 2010 / 01 / 12 & 02: 24: 33.639 & 33.185 & -115.569 & 3.7 \\ 2010 / 01 / 12 & 04: 06: 43.639 & 33.193 & -115.615 & 3.9 \\ 2010 / 01 / 12 & 04: 18: 24.639 & 33.174 & -115.603 & 0.1 \\ 2010 / 01 / 12 & 04: 36: 12.639 & 33.175 & -115.603 & 0.1 \\ 2010 / 01 / 12 & 04: 44: 09.639 & 33.175 & -115.603 & 0.1 \\ 2010 / 01 / 12 & 06: 27: 12.639 & 33.175 & -115.642 & 0.1 \\ 2010 / 01 / 12 & 06: 27: 28.639 & 33.175 & -115.601 & 0.1 \\ 201 / 12 & 07: 08: 47.639 & 33.193 & -115.615 & 3.9 \\ 07: 17: 37.639 & 33.176 & -115.608 & 0.4\end{array}$




$\begin{array}{lllll}2010 / 01 / 12 & 07: 53: 30.639 & 33.180 & -115.603 & 0.1 \\ 2010 / 01 / 12 & 09: 51: 28.639 & 33.158 & -115.642 & 0.1 \\ 2010 / 01 / 12 & 09: 52: 35.639 & 33.158 & -115.642 & 0.1 \\ 2010 / 01 / 12 & 11: 19: 45.639 & 33.179 & -115.597 & 0.1 \\ 2010 / 01 / 12 & 12: 07: 03.639 & 33.174 & -115.603 & 0.1 \\ 2010 / 01 / 12 & 13: 36: 47.639 & 33.181 & -115.608 & 1.0 \\ 2010 / 01 / 12 & 13: 56: 36.639 & 33.176 & -115.608 & 0.4 \\ 2010 / 01 / 12 & 15: 16: 21.639 & 33.108 & -115.602 & 8.1 \\ 2010 / 01 / 12 & 15: 17: 14.639 & 33.193 & -115.615 & 3.9 \\ 2010 / 01 / 12 & 15: 17: 35.639 & 33.108 & -115.602 & 8.1 \\ 2010 / 01 / 12 & 15: 18: 17.639 & 33.118 & -115.590 & 13.8 \\ 2010 / 01 / 12 & 15: 19: 43.639 & 33.160 & -115.633 & 4.6 \\ 2010 / 01 / 12 & 15: 34: 55.639 & 33.100 & -115.590 & 14.6 \\ 2010 / 01 / 12 & 16: 24: 36.639 & 33.176 & -115.608 & 0.4 \\ 2010 / 01 / 12 & 16: 25: 00.639 & 33.179 & -115.594 & 1.5 \\ 2010 / 01 / 12 & 17: 27: 06.639 & 33.181 & -115.603 & 1.4 \\ 2010 / 01 / 12 & 19: 57: 55.639 & 33.176 & -115.608 & 0.4 \\ 2010 / 01 / 12 & 19: 58: 16.639 & 33.160 & -115.642 & 1.5 \\ 2010 / 01 / 12 & 19: 58: 32.639 & 33.177 & -115.606 & 0.1 \\ 2010 / 01 / 12 & 20: 00: 30.639 & 33.176 & -115.608 & 0.4 \\ 2010 / 01 / 12 & 20: 02: 12.639 & 33.176 & -115.608 & 0.4 \\ 2010 / 01 / 12 & 21: 54: 35.639 & 33.181 & -115.659 & 6.0 \\ 2010 / 01 / 12 & 23: 46: 47.639 & 33.108 & -115.602 & 8.1 \\ 2010 / 01 / 13 & 00: 25: 34.639 & 33.180 & -115.600 & 0.1 \\ 2010 / 01 / 13 & 00: 41: 46.639 & 33.193 & -115.583 & 5.9 \\ 2010 / 01 / 13 & 01: 13: 59.639 & 33.176 & -115.604 & 0.5 \\ 2010 / 01 / 13 & 02: 42: 25.639 & 33.175 & -115.601 & 0.1 \\ 2010 / 01 / 13 & 02: 46: 47.639 & 33.176 & -115.604 & 0.5 \\ 2010 / 01 / 13 & 03: 16: 19.639 & 33.166 & -115.617 & 5.3 \\ 2010 / 01 / 13 & 03: 22: 50.639 & 33.197 & -115.596 & 3.0 \\ 2010 / 01 / 13 & 03: 38: 40.639 & 33.209 & -115.590 & 3.1 \\ 2010 / 01 / 13 & 03: 40: 08.639 & 33.209 & -115.590 & 3.1 \\ 2010 / 01 / 13 & 05: 12: 32.639 & 33.108 & -115.602 & 8.1 \\ 2010 / 01 / 13 & 05: 14: 07.639 & 33.175 & -115.600 & 1.1 \\ 2010 / 01 / 13 & 05: 26: 55.639 & 33.196 & -115.559 & 0.2 \\ 2010 / 01 / 13 & 09: 27: 49.639 & 33.179 & -115.597 & 0.1 \\ 2010 / 01 / 13 & 10: 02: 57.639 & 33.229 & -115.576 & 3.4 \\ 2010 / 01 / 13 & 10: 43: 57.639 & 33.180 & -115.603 & 0.1 \\ 2010 / 01 / 13 & 10: 44: 36.639 & 33.179 & -115.614 & 1.8 \\ 2010 / 01 / 13 & 10: 44: 53.639 & 33.198 & -115.593 & 2.3 \\ 2010 / 01 / 13 & 10: 45: 08.639 & 33.180 & -115.603 & 0.1 \\ 2010 / 01 / 13 & 11: 02: 28.639 & 33.179 & -115.594 & 1.5 \\ 2010 / 01 / 13 & 12: 44: 14.639 & 33.179 & -115.597 & 0.1 \\ 2010 / 01 / 13 & 12: 44: 47.639 & 33.203 & -115.578 & 2.2 \\ 2010 / 01 / 13 & 12: 45: 07.639 & 33.182 & -115.602 & 2.5 \\ 2010 / 01 / 13 & 12: 45: 27.639 & 33.176 & -115.618 & 3.7\end{array}$




$\begin{array}{lllll}2010 / 01 / 13 & 13: 12: 08.639 & 33.177 & -115.606 & 0.1 \\ 2010 / 01 / 13 & 13: 12: 38.639 & 33.176 & -115.608 & 0.4 \\ 2010 / 01 / 13 & 13: 13: 08.639 & 33.181 & -115.611 & 0.4 \\ 2010 / 01 / 13 & 13: 13: 35.639 & 33.177 & -115.606 & 0.1 \\ 2010 / 01 / 13 & 13: 59: 24.639 & 33.229 & -115.576 & 3.4 \\ 2010 / 01 / 13 & 14: 14: 28.639 & 33.175 & -115.610 & 2.4 \\ 2010 / 01 / 13 & 14: 58: 49.639 & 33.174 & -115.603 & 0.1 \\ 2010 / 01 / 13 & 16: 29: 15.639 & 33.176 & -115.602 & 3.2 \\ 2010 / 01 / 13 & 16: 40: 23.639 & 33.176 & -115.602 & 3.2 \\ 2010 / 01 / 13 & 16: 47: 46.639 & 33.181 & -115.603 & 1.4 \\ 2010 / 01 / 13 & 16: 48: 00.639 & 33.175 & -115.603 & 0.1 \\ 2010 / 01 / 13 & 16: 54: 03.639 & 33.192 & -115.572 & 1.6 \\ 2010 / 01 / 13 & 16: 54: 39.639 & 33.176 & -115.602 & 3.2 \\ 2010 / 01 / 13 & 16: 55: 21.639 & 33.179 & -115.594 & 1.5 \\ 2010 / 01 / 13 & 16: 57: 22.639 & 33.177 & -115.606 & 0.1 \\ 2010 / 01 / 13 & 17: 54: 21.639 & 33.181 & -115.665 & 4.2 \\ 2010 / 01 / 13 & 18: 03: 51.639 & 33.181 & -115.665 & 4.2 \\ 2010 / 01 / 13 & 18: 04: 07.639 & 33.118 & -115.610 & 9.1 \\ 2010 / 01 / 13 & 19: 47: 28.639 & 33.172 & -115.622 & 4.0 \\ 2010 / 01 / 13 & 20: 58: 46.639 & 33.176 & -115.608 & 0.4 \\ 2010 / 01 / 13 & 22: 34: 18.639 & 33.180 & -115.603 & 0.1 \\ 2010 / 01 / 13 & 23: 13: 18.639 & 33.190 & -115.604 & 1.2 \\ 2010 / 01 / 13 & 23: 53: 35.639 & 33.179 & -115.594 & 1.5 \\ 2010 / 01 / 14 & 01: 38: 45.639 & 33.182 & -115.602 & 2.5 \\ 2010 / 01 / 14 & 02: 09: 50.639 & 33.177 & -115.635 & 5.3 \\ 2010 / 01 / 14 & 02: 33: 10.639 & 33.174 & -115.603 & 0.1 \\ 2010 / 01 / 14 & 02: 35: 53.639 & 33.174 & -115.603 & 0.1 \\ 2010 / 01 / 14 & 03: 01: 37.639 & 33.157 & -115.668 & 0.1 \\ 2010 / 01 / 14 & 03: 01: 53.639 & 33.157 & -115.668 & 0.1 \\ 2010 / 01 / 14 & 03: 50: 13.639 & 33.175 & -115.601 & 0.1 \\ 2010 / 01 / 14 & 07: 25: 28.639 & 33.175 & -115.600 & 1.1 \\ 2010 / 01 / 14 & 07: 58: 52.639 & 33.176 & -115.608 & 0.4 \\ 2010 / 01 / 14 & 07: 59: 33.639 & 33.176 & -115.608 & 0.4 \\ 2010 / 01 / 14 & 08: 04: 01.639 & 33.176 & -115.608 & 0.4 \\ 2010 / 01 / 14 & 08: 21: 03.639 & 33.181 & -115.659 & 6.0 \\ 2010 / 01 / 14 & 08: 41: 53.639 & 33.179 & -115.597 & 0.1 \\ 2010 / 01 / 14 & 09: 28: 00.639 & 33.179 & -115.597 & 0.1 \\ 2010 / 01 / 14 & 09: 47: 59.639 & 33.184 & -115.606 & 2.9 \\ 2010 / 01 / 14 & 09: 48: 45.639 & 33.184 & -115.606 & 2.9 \\ 2010 / 01 / 14 & 09: 51: 30.639 & 33.179 & -115.597 & 0.1 \\ 2010 / 01 / 14 & 13: 43: 06.639 & 33.175 & -115.600 & 1.1 \\ 2010 / 01 / 14 & 13: 51: 38.639 & 33.175 & -115.610 & 2.4 \\ 2010 / 01 / 14 & 18: 12: 01.639 & 33.188 & -115.621 & 4.8 \\ 20: 14 & 20: 06.639 & 33.180 & -115.603 & 0.1\end{array}$




$\begin{array}{lllll}2010 / 01 / 14 & 20: 57: 00.639 & 33.176 & -115.609 & 2.1 \\ 2010 / 01 / 14 & 22: 36: 24.639 & 33.174 & -115.603 & 0.1 \\ 2010 / 01 / 14 & 23: 00: 09.639 & 33.176 & -115.618 & 3.7 \\ 2010 / 01 / 14 & 23: 11: 41.639 & 33.176 & -115.604 & 0.0 \\ 2010 / 01 / 15 & 00: 34: 11.639 & 33.234 & -115.604 & 4.8 \\ 2010 / 01 / 15 & 00: 43: 56.639 & 33.176 & -115.604 & 0.5 \\ 2010 / 01 / 15 & 00: 54: 27.639 & 33.193 & -115.583 & 5.9 \\ 2010 / 01 / 15 & 01: 18: 40.639 & 33.181 & -115.608 & 1.0 \\ 2010 / 01 / 15 & 01: 19: 14.639 & 33.181 & -115.608 & 1.0 \\ 2010 / 01 / 15 & 01: 20: 03.639 & 33.176 & -115.604 & 0.5 \\ 2010 / 01 / 15 & 01: 23: 26.639 & 33.176 & -115.608 & 0.4 \\ 2010 / 01 / 15 & 01: 24: 27.639 & 33.182 & -115.602 & 2.5 \\ 2010 / 01 / 15 & 01: 25: 06.639 & 33.193 & -115.583 & 5.9 \\ 2010 / 01 / 15 & 01: 58: 54.639 & 33.175 & -115.603 & 0.1 \\ 2010 / 01 / 15 & 01: 59: 54.639 & 33.175 & -115.603 & 0.1 \\ 2010 / 01 / 15 & 02: 01: 32.639 & 33.175 & -115.603 & 0.1 \\ 2010 / 01 / 15 & 02: 02: 39.639 & 33.175 & -115.603 & 0.1 \\ 2010 / 01 / 15 & 02: 04: 00.639 & 33.181 & -115.603 & 2.0 \\ 2010 / 01 / 15 & 02: 04: 27.639 & 33.170 & -115.600 & 1.1 \\ 2010 / 01 / 15 & 02: 05: 30.639 & 33.176 & -115.604 & 0.5 \\ 2010 / 01 / 15 & 02: 08: 50.639 & 33.177 & -115.635 & 5.3 \\ 2010 / 01 / 15 & 02: 09: 48.639 & 33.180 & -115.600 & 0.1 \\ 2010 / 01 / 15 & 02: 11: 06.639 & 33.180 & -115.600 & 0.1 \\ 2010 / 01 / 15 & 02: 12: 54.639 & 33.180 & -115.600 & 0.1 \\ 2010 / 01 / 15 & 02: 13: 50.639 & 33.181 & -115.601 & 0.7 \\ 2010 / 01 / 15 & 02: 14: 29.639 & 33.175 & -115.600 & 1.1 \\ 2010 / 01 / 15 & 02: 15: 26.639 & 33.188 & -115.598 & 2.3 \\ 2010 / 01 / 15 & 02: 15: 46.639 & 33.212 & -115.633 & 7.6 \\ 2010 / 01 / 15 & 02: 16: 05.639 & 33.181 & -115.602 & 0.4 \\ 2010 / 01 / 15 & 02: 16: 21.639 & 33.182 & -115.612 & 2.2 \\ 2010 / 01 / 15 & 02: 17: 00.639 & 33.181 & -115.603 & 2.0 \\ 2010 / 01 / 15 & 02: 17: 41.639 & 33.184 & -115.610 & 2.8 \\ 2010 / 01 / 15 & 02: 19: 50.639 & 33.182 & -115.603 & 0.1 \\ 2010 / 01 / 15 & 02: 20: 28.639 & 33.182 & -115.603 & 0.1 \\ 2010 / 01 / 15 & 02: 21: 07.639 & 33.175 & -115.603 & 0.1 \\ 2010 / 01 / 15 & 02: 22: 12.639 & 33.177 & -115.635 & 5.3 \\ 2010 / 01 / 15 & 02: 22: 26.639 & 33.181 & -115.602 & 0.4 \\ 2010 / 01 / 15 & 02: 23: 10.639 & 33.179 & -115.597 & 0.1 \\ 2010 / 01 / 15 & 02: 23: 23.639 & 33.188 & -115.609 & 2.3 \\ 2010 / 01 / 15 / 01 / 15 & 02: 23: 55.639 & 33.181 & -115.603 & 0.1 \\ 2010 / 01 / 15 & 02: 24: 41.639 & 33.188 & -115.609 & 2.3 \\ 201 / 15 & 02: 25: 49.639 & 33.180 & -115.600 & 0.1 \\ 203.603 & 02: 26: 46.639 & 33.188 & -115.609 & 2.3 \\ 201: 11.639 & 33.180 & -115.603 & 0.1\end{array}$




$\begin{array}{lllll}2010 / 01 / 15 & 02: 27: 34.639 & 33.177 & -115.606 & 0.1 \\ 2010 / 01 / 15 & 02: 27: 52.639 & 33.188 & -115.609 & 2.3 \\ 2010 / 01 / 15 & 02: 29: 36.639 & 33.186 & -115.604 & 2.0 \\ 2010 / 01 / 15 & 02: 29: 48.639 & 33.176 & -115.608 & 0.4 \\ 2010 / 01 / 15 & 02: 30: 12.639 & 33.184 & -115.610 & 2.8 \\ 2010 / 01 / 15 & 02: 30: 34.639 & 33.179 & -115.602 & 0.6 \\ 2010 / 01 / 15 & 02: 30: 54.639 & 33.182 & -115.602 & 2.5 \\ 2010 / 01 / 15 & 02: 31: 26.639 & 33.158 & -115.629 & 6.0 \\ 2010 / 01 / 15 & 02: 32: 35.639 & 33.180 & -115.603 & 0.1 \\ 2010 / 01 / 15 & 02: 33: 13.639 & 33.184 & -115.597 & 0.1 \\ 2010 / 01 / 15 & 02: 33: 50.639 & 33.181 & -115.603 & 1.4 \\ 2010 / 01 / 15 & 02: 35: 05.639 & 33.198 & -115.593 & 2.3 \\ 2010 / 01 / 15 & 02: 36: 23.639 & 33.187 & -115.601 & 1.7 \\ 2010 / 01 / 15 & 02: 37: 13.639 & 33.180 & -115.600 & 0.1 \\ 2010 / 01 / 15 & 02: 37: 51.639 & 33.188 & -115.609 & 2.3 \\ 2010 / 01 / 15 & 02: 38: 30.639 & 33.175 & -115.603 & 0.1 \\ 2010 / 01 / 15 & 02: 39: 20.639 & 33.179 & -115.597 & 0.1 \\ 2010 / 01 / 15 & 02: 39: 59.639 & 33.176 & -115.608 & 0.4 \\ 2010 / 01 / 15 & 02: 41: 32.639 & 33.187 & -115.601 & 1.7 \\ 2010 / 01 / 15 & 02: 42: 14.639 & 33.177 & -115.606 & 0.1 \\ 2010 / 01 / 15 & 02: 42: 34.639 & 33.175 & -115.603 & 0.1 \\ 2010 / 01 / 15 & 02: 43: 36.639 & 33.182 & -115.603 & 0.1 \\ 2010 / 01 / 15 & 02: 44: 52.639 & 33.177 & -115.635 & 5.3 \\ 2010 / 01 / 15 & 02: 45: 12.639 & 33.175 & -115.603 & 0.1 \\ 2010 / 01 / 15 & 02: 45: 30.639 & 33.182 & -115.602 & 2.5 \\ 2010 / 01 / 15 & 02: 46: 53.639 & 33.175 & -115.603 & 0.1 \\ 2010 / 01 / 15 & 02: 47: 05.639 & 33.188 & -115.609 & 2.3 \\ 2010 / 01 / 15 & 02: 47: 34.639 & 33.175 & -115.610 & 2.4 \\ 2010 / 01 / 15 & 02: 47: 53.639 & 33.181 & -115.602 & 0.4 \\ 2010 / 01 / 15 & 02: 48: 24.639 & 33.179 & -115.602 & 0.6 \\ 2010 / 01 / 15 & 02: 48: 49.639 & 33.180 & -115.600 & 0.1 \\ 2010 / 01 / 15 & 02: 49: 27.639 & 33.185 & -115.608 & 2.0 \\ 2010 / 01 / 15 & 02: 50: 31.639 & 33.177 & -115.606 & 0.1 \\ 2010 / 01 / 15 & 02: 51: 46.639 & 33.181 & -115.603 & 1.4 \\ 2010 / 01 / 15 & 02: 54: 47.639 & 33.180 & -115.603 & 0.1 \\ 2010 / 01 / 15 & 02: 57: 23.639 & 33.181 & -115.603 & 2.0 \\ 2010 / 01 / 15 & 02: 59: 50.639 & 33.179 & -115.602 & 0.6 \\ 2010 / 01 / 15 & 03: 02: 47.639 & 33.179 & -115.602 & 0.6 \\ 2010 / 01 / 15 & 03: 04: 39.639 & 33.188 & -115.609 & 2.3 \\ 2010 / 01 / 15 & 03: 06: 10.639 & 33.184 & -115.610 & 2.8 \\ 2010 / 01 / 15 & 03: 07: 04.639 & 33.181 & -115.659 & 6.0 \\ 201 / 15 & 03: 07: 52.639 & 33.188 & -115.609 & 2.3 \\ 203 & 03: 08: 08.639 & 33.185 & -115.608 & 2.0 \\ 203: 08: 59.639 & 33.186 & -115.604 & 2.0\end{array}$




\begin{tabular}{|c|c|c|c|}
\hline $2010 / 01 / 15$ & $03: 12: 10.639$ & 33.181 & -115.603 \\
\hline 2010/01/15 & $03: 14: 16.639$ & 33.188 & -115.609 \\
\hline 2010/01/15 & $03: 15: 05.639$ & 33.176 & -115.609 \\
\hline 2010/01/15 & 03:18:01.639 & 33.190 & -115.604 \\
\hline 2010/01/15 & $03: 20: 20.639$ & 33.198 & -115.593 \\
\hline 2010/01/15 & $03: 21: 27.639$ & 33.181 & -115.601 \\
\hline 2010/01/15 & $03: 22: 35.639$ & 33.184 & -115.610 \\
\hline 2010/01/15 & $03: 23: 47.639$ & 33.179 & -115.602 \\
\hline 2010/01/15 & $03: 24: 15.639$ & 33.182 & -115.612 \\
\hline 2010/01/15 & 03:26:09.639 & 33.181 & -115.603 \\
\hline 2010/01/15 & $03: 27: 12.639$ & 33.198 & -115.593 \\
\hline 2010/01/15 & $03: 28: 57.639$ & 33.181 & -115.603 \\
\hline 2010/01/15 & $03: 29: 57.639$ & 33.181 & -115.603 \\
\hline 2010/01/15 & $03: 30: 56.639$ & 33.190 & -115.604 \\
\hline 2010/01/15 & $03: 32: 12.639$ & 33.186 & -115.604 \\
\hline 2010/01/15 & $03: 33: 53.639$ & 33.190 & -115.604 \\
\hline 2010/01/15 & $03: 34: 39.639$ & 33.190 & -115.604 \\
\hline 2010/01/15 & $03: 35: 31.639$ & 33.212 & -115.633 \\
\hline 2010/01/15 & $03: 36: 17.639$ & 33.175 & -115.603 \\
\hline 2010/01/15 & 03:38:39.639 & 33.187 & -115.601 \\
\hline 2010/01/15 & $03: 39: 22.639$ & 33.188 & -115.609 \\
\hline 2010/01/15 & $03: 39: 42.639$ & 33.190 & -115.604 \\
\hline 2010/01/15 & $03: 40: 56.639$ & 33.158 & -115.629 \\
\hline 2010/01/15 & $03: 44: 10.639$ & 33.188 & -115.600 \\
\hline 2010/01/15 & 03:44:29.639 & 33.190 & -115.604 \\
\hline 2010/01/15 & $03: 47: 55.639$ & 33.177 & -115.635 \\
\hline 2010/01/15 & $03: 49: 38.639$ & 33.176 & -115.604 \\
\hline 2010/01/15 & $03: 49: 57.639$ & 33.181 & -115.665 \\
\hline 2010/01/15 & $03: 50: 30.639$ & 33.180 & -115.603 \\
\hline 2010/01/15 & $03: 58: 43.639$ & 33.117 & -115.613 \\
\hline 2010/01/15 & 04:00:11.639 & 33.181 & -115.603 \\
\hline 2010/01/15 & $04: 02: 22.639$ & 33.188 & -115.609 \\
\hline $2010 / 01 / 15$ & 04:07:33.639 & 33.181 & -115.601 \\
\hline 2010/01/15 & 04:08:08.639 & 33.180 & -115.600 \\
\hline $2010 / 01 / 15$ & $04: 12: 33.639$ & 33.179 & -115.594 \\
\hline $2010 / 01 / 15$ & $04: 13: 35.639$ & 33.198 & -115.593 \\
\hline $2010 / 01 / 15$ & $04: 21: 45.639$ & 33.181 & -115.601 \\
\hline 2010/01/15 & $04: 22: 20.639$ & 33.212 & -115.633 \\
\hline $2010 / 01 / 15$ & $04: 26: 14.639$ & 33.179 & -115.602 \\
\hline $2010 / 01 / 15$ & $04: 31: 50.639$ & 33.176 & -115.608 \\
\hline $2010 / 01 / 15$ & $04: 32: 24.639$ & 33.229 & -115.576 \\
\hline 2010/01/15 & 04:35:08.639 & 33.180 & -115.603 \\
\hline 2010/01/15 & $04: 37: 22.639$ & 33.199 & -115.617 \\
\hline 2010/01/15 & $04: 44: 24.639$ & 33.190 & -115.604 \\
\hline $2010 / 01 / 15$ & $04: 44: 49.639$ & 33.185 & -115.608 \\
\hline $2010 / 01 / 15$ & $04: 45: 34.639$ & 33.190 & -115.604 \\
\hline
\end{tabular}




$\begin{array}{lllll}2010 / 01 / 15 & 04: 45: 53.639 & 33.190 & -115.604 & 1.2 \\ 2010 / 01 / 15 & 04: 47: 03.639 & 33.176 & -115.608 & 0.4 \\ 2010 / 01 / 15 & 04: 47: 34.639 & 33.190 & -115.604 & 1.2 \\ 2010 / 01 / 15 & 04: 48: 14.639 & 33.182 & -115.603 & 0.1 \\ 2010 / 01 / 15 & 04: 48: 32.639 & 33.182 & -115.612 & 2.2 \\ 2010 / 01 / 15 & 04: 50: 49.639 & 33.184 & -115.610 & 2.8 \\ 2010 / 01 / 15 & 04: 51: 40.639 & 33.197 & -115.596 & 3.0 \\ 2010 / 01 / 15 & 04: 57: 03.639 & 33.184 & -115.610 & 2.8 \\ 2010 / 01 / 15 & 05: 00: 48.639 & 33.181 & -115.665 & 4.2 \\ 2010 / 01 / 15 & 05: 21: 29.639 & 33.182 & -115.603 & 0.1 \\ 2010 / 01 / 15 & 05: 23: 12.639 & 33.181 & -115.601 & 0.7 \\ 2010 / 01 / 15 & 05: 23: 54.639 & 33.181 & -115.601 & 0.7 \\ 2010 / 01 / 15 & 05: 30: 16.639 & 33.179 & -115.602 & 0.6 \\ 2010 / 01 / 15 & 05: 32: 45.639 & 33.181 & -115.603 & 2.0 \\ 2010 / 01 / 15 & 05: 33: 17.639 & 33.188 & -115.600 & 0.1 \\ 2010 / 01 / 15 & 05: 34: 10.639 & 33.188 & -115.609 & 2.3 \\ 2010 / 01 / 15 & 05: 34: 45.639 & 33.188 & -115.600 & 0.1 \\ 2010 / 01 / 15 & 05: 35: 19.639 & 33.181 & -115.603 & 1.4 \\ 2010 / 01 / 15 & 05: 38: 32.639 & 33.175 & -115.603 & 0.1 \\ 2010 / 01 / 15 & 05: 43: 02.639 & 33.180 & -115.603 & 0.1 \\ 2010 / 01 / 15 & 05: 44: 42.639 & 33.182 & -115.612 & 2.2 \\ 2010 / 01 / 15 & 05: 52: 33.639 & 33.181 & -115.601 & 0.7 \\ 2010 / 01 / 15 & 05: 55: 57.639 & 33.179 & -115.602 & 0.6 \\ 2010 / 01 / 15 & 05: 58: 48.639 & 33.177 & -115.606 & 0.1 \\ 2010 / 01 / 15 & 06: 10: 21.639 & 33.179 & -115.597 & 0.1 \\ 2010 / 01 / 15 & 06: 12: 59.639 & 33.188 & -115.609 & 2.3 \\ 2010 / 01 / 15 & 06: 16: 50.639 & 33.184 & -115.610 & 2.8 \\ 2010 / 01 / 15 & 06: 19: 25.639 & 33.179 & -115.602 & 0.6 \\ 2010 / 01 / 15 & 06: 20: 13.639 & 33.181 & -115.603 & 0.1 \\ 2010 / 01 / 15 & 06: 21: 52.639 & 33.188 & -115.599 & 0.8 \\ 2010 / 01 / 15 & 06: 23: 50.639 & 33.181 & -115.603 & 0.1 \\ 2010 / 01 / 15 & 06: 24: 52.639 & 33.179 & -115.594 & 1.5 \\ 2010 / 01 / 15 & 06: 26: 58.639 & 33.208 & -115.582 & 2.5 \\ 2010 / 01 / 15 & 06: 29: 52.639 & 33.187 & -115.601 & 1.7 \\ 2010 / 01 / 15 & 06: 37: 40.639 & 33.179 & -115.602 & 0.6 \\ 2010 / 01 / 15 & 07: 14: 37.639 & 33.181 & -115.665 & 4.2 \\ 2010 / 01 / 15 & 07: 20: 13.639 & 33.176 & -115.602 & 3.2 \\ 2010 / 01 / 15 & 07: 28: 21.639 & 33.181 & -115.665 & 4.2 \\ 2010 / 01 / 15 & 07: 35: 14.639 & 33.186 & -115.604 & 2.0 \\ 2010 / 01 / 15 & 07: 37: 44.639 & 33.183 & -115.607 & 0.0 \\ 201 / 15 & 07: 42: 14.639 & 33.186 & -115.604 & 2.0 \\ 201 / 15 & 08: 00: 26.639 & 33.188 & -115.609 & 2.2 \\ 208 & 08: 02: 16.639 & 33.188 & -115.609 & 2.3 \\ 208: 06: 12.639 & 33.188 & -115.598 & 2.3 \\ 203: 01.639 & 33.188 & -115.609 & 2.3\end{array}$




$\begin{array}{lllll}2010 / 01 / 15 & 08: 10: 27.639 & 33.188 & -115.609 & 2.3 \\ 2010 / 01 / 15 & 08: 12: 51.639 & 33.180 & -115.603 & 0.1 \\ 2010 / 01 / 15 & 08: 15: 49.639 & 33.184 & -115.610 & 2.8 \\ 2010 / 01 / 15 & 08: 16: 54.639 & 33.188 & -115.600 & 0.1 \\ 2010 / 01 / 15 & 08: 19: 32.639 & 33.188 & -115.609 & 2.3 \\ 2010 / 01 / 15 & 08: 20: 22.639 & 33.182 & -115.618 & 3.1 \\ 2010 / 01 / 15 & 08: 20: 48.639 & 33.190 & -115.608 & 2.2 \\ 2010 / 01 / 15 & 08: 42: 49.639 & 33.182 & -115.603 & 0.1 \\ 2010 / 01 / 15 & 08: 47: 56.639 & 33.185 & -115.608 & 2.0 \\ 2010 / 01 / 15 & 08: 51: 22.639 & 33.188 & -115.609 & 2.3 \\ 2010 / 01 / 15 & 08: 53: 53.639 & 33.182 & -115.603 & 0.1 \\ 2010 / 01 / 15 & 09: 20: 22.639 & 33.188 & -115.609 & 2.3 \\ 2010 / 01 / 15 & 10: 11: 10.639 & 33.187 & -115.604 & 3.2 \\ 2010 / 01 / 15 & 10: 26: 03.639 & 33.182 & -115.603 & 0.1 \\ 2010 / 01 / 15 & 10: 27: 03.639 & 33.187 & -115.601 & 1.7 \\ 2010 / 01 / 15 & 10: 35: 45.639 & 33.181 & -115.602 & 0.4 \\ 2010 / 01 / 15 & 10: 43: 22.639 & 33.148 & -115.633 & 4.3 \\ 2010 / 01 / 15 & 10: 51: 19.639 & 33.182 & -115.603 & 0.1 \\ 2010 / 01 / 15 & 10: 57: 44.639 & 33.184 & -115.610 & 2.8 \\ 2010 / 01 / 15 & 10: 58: 24.639 & 33.181 & -115.602 & 0.4 \\ 2010 / 01 / 15 & 11: 07: 18.639 & 33.175 & -115.610 & 2.4 \\ 2010 / 01 / 15 & 11: 20: 20.639 & 33.188 & -115.600 & 0.1 \\ 2010 / 01 / 15 & 11: 27: 47.639 & 33.181 & -115.603 & 0.1 \\ 2010 / 01 / 15 & 11: 28: 29.639 & 33.188 & -115.609 & 2.3 \\ 2010 / 01 / 15 & 11: 29: 21.639 & 33.196 & -115.587 & 5.3 \\ 2010 / 01 / 15 & 11: 30: 11.639 & 33.185 & -115.608 & 2.0 \\ 2010 / 01 / 15 & 11: 30: 47.639 & 33.188 & -115.609 & 2.3 \\ 2010 / 01 / 15 & 11: 31: 04.639 & 33.187 & -115.604 & 3.2 \\ 2010 / 01 / 15 & 11: 31: 46.639 & 33.199 & -115.571 & 3.3 \\ 2010 / 01 / 15 & 11: 33: 02.639 & 33.187 & -115.604 & 3.2 \\ 2010 / 01 / 15 & 11: 33: 13.639 & 33.187 & -115.604 & 3.2 \\ 2010 / 01 / 15 & 11: 34: 50.639 & 33.188 & -115.600 & 0.1 \\ 2010 / 01 / 15 & 11: 35: 04.639 & 33.186 & -115.593 & 0.1 \\ 2010 / 01 / 15 & 11: 35: 54.639 & 33.183 & -115.617 & 4.3 \\ 2010 / 01 / 15 & 11: 36: 29.639 & 33.187 & -115.604 & 3.2 \\ 2010 / 01 / 15 & 11: 37: 31.639 & 33.187 & -115.604 & 3.2 \\ 2010 / 01 / 15 & 11: 41: 47.639 & 33.184 & -115.604 & 0.1 \\ 2010 / 01 / 15 & 11: 43: 35.639 & 33.187 & -115.604 & 3.2 \\ 2010 / 01 / 15 & 11: 44: 07.639 & 33.188 & -115.609 & 2.3 \\ 2010 / 01 / 15 & 11: 50: 10.639 & 33.248 & -115.633 & 2.9 \\ 2010 / 01 / 15 & 11: 52: 34.639 & 33.184 & -115.609 & 5.7 \\ 201 / 15 & 11: 57: 06.639 & 33.179 & -115.594 & 1.5 \\ 201 / 15 & 11: 58: 38.639 & 33.181 & -115.603 & 0.1 \\ 2: 00: 17.639 & 33.179 & -115.594 & 1.5\end{array}$




$\begin{array}{lllll}2010 / 01 / 15 & 12: 01: 50.639 & 33.181 & -115.602 & 0.4 \\ 2010 / 01 / 15 & 12: 16: 19.639 & 33.214 & -115.620 & 5.3 \\ 2010 / 01 / 15 & 12: 19: 11.639 & 33.234 & -115.604 & 4.8 \\ 2010 / 01 / 15 & 12: 23: 40.639 & 33.177 & -115.621 & 2.7 \\ 2010 / 01 / 15 & 12: 24: 04.639 & 33.181 & -115.665 & 4.2 \\ 2010 / 01 / 15 & 12: 38: 17.639 & 33.179 & -115.602 & 0.6 \\ 2010 / 01 / 15 & 12: 38: 33.639 & 33.212 & -115.633 & 7.6 \\ 2010 / 01 / 15 & 12: 45: 07.639 & 33.196 & -115.587 & 5.3 \\ 2010 / 01 / 15 & 12: 47: 43.639 & 33.188 & -115.609 & 2.3 \\ 2010 / 01 / 15 & 12: 56: 18.639 & 33.184 & -115.610 & 2.8 \\ 2010 / 01 / 15 & 13: 05: 59.639 & 33.181 & -115.602 & 0.4 \\ 2010 / 01 / 15 & 13: 10: 16.639 & 33.188 & -115.600 & 0.1 \\ 2010 / 01 / 15 & 13: 14: 11.639 & 33.188 & -115.609 & 2.3 \\ 2010 / 01 / 15 & 13: 15: 51.639 & 33.179 & -115.614 & 1.8 \\ 2010 / 01 / 15 & 13: 18: 57.639 & 33.184 & -115.609 & 2.2 \\ 2010 / 01 / 15 & 13: 26: 48.639 & 33.188 & -115.609 & 2.3 \\ 2010 / 01 / 15 & 13: 34: 18.639 & 33.192 & -115.572 & 1.6 \\ 2010 / 01 / 15 & 13: 52: 29.639 & 33.193 & -115.632 & 3.5 \\ 2010 / 01 / 15 & 14: 07: 04.639 & 33.184 & -115.610 & 2.8 \\ 2010 / 01 / 15 & 14: 32: 31.639 & 33.182 & -115.602 & 2.5 \\ 2010 / 01 / 15 & 14: 46: 13.639 & 33.192 & -115.572 & 1.6 \\ 2010 / 01 / 15 & 14: 54: 22.639 & 33.186 & -115.604 & 2.0 \\ 2010 / 01 / 15 & 16: 09: 57.639 & 33.181 & -115.602 & 0.4 \\ 2010 / 01 / 15 & 16: 14: 37.639 & 33.187 & -115.601 & 1.7 \\ 2010 / 01 / 15 & 16: 27: 57.639 & 33.184 & -115.604 & 0.1 \\ 2010 / 01 / 15 & 16: 30: 42.639 & 33.184 & -115.604 & 0.1 \\ 2010 / 01 / 15 & 16: 31: 17.639 & 33.184 & -115.604 & 0.1 \\ 2010 / 01 / 15 & 16: 31: 35.639 & 33.184 & -115.604 & 0.1 \\ 2010 / 01 / 15 & 16: 32: 12.639 & 33.184 & -115.604 & 0.1 \\ 2010 / 01 / 15 & 16: 33: 33.639 & 33.188 & -115.609 & 2.3 \\ 2010 / 01 / 15 & 16: 43: 16.639 & 33.184 & -115.604 & 0.1 \\ 2010 / 01 / 15 & 17: 05: 38.639 & 33.181 & -115.665 & 4.2 \\ 2010 / 01 / 15 & 17: 14: 22.639 & 33.181 & -115.603 & 1.4 \\ 2010 / 01 / 15 & 17: 20: 35.639 & 33.186 & -115.604 & 2.0 \\ 2010 / 01 / 15 & 17: 43: 16.639 & 33.198 & -115.593 & 2.3 \\ 2010 / 01 / 15 & 17: 47: 04.639 & 33.147 & -115.623 & 7.9 \\ 2010 / 01 / 15 & 18: 05: 16.639 & 33.185 & -115.608 & 2.0 \\ 2010 / 01 / 15 & 18: 57: 43.639 & 33.184 & -115.608 & 0.3 \\ 2010 / 01 / 15 & 19: 26: 09.639 & 33.184 & -115.608 & 0.3 \\ 2010 / 01 / 15 & 19: 34: 42.639 & 33.177 & -115.621 & 2.7 \\ 2010 / 01 / 15 & 19: 37: 32.639 & 33.214 & -115.620 & 5.3 \\ 2010 / 01 / 15 & 19: 44: 13.639 & 33.186 & -115.604 & 2.0 \\ & 19: 51: 33.639 & 33.182 & -115.618 & 3.1 \\ 20: 23: 31.639 & 33.179 & -115.602 & 0.6\end{array}$




$\begin{array}{lllll}2010 / 01 / 15 & 20: 43: 51.639 & 33.188 & -115.609 & 2.3 \\ 2010 / 01 / 15 & 20: 47: 24.639 & 33.179 & -115.602 & 0.6 \\ 2010 / 01 / 15 & 20: 50: 48.639 & 33.179 & -115.614 & 1.8 \\ 2010 / 01 / 15 & 20: 55: 31.639 & 33.186 & -115.604 & 2.0 \\ 2010 / 01 / 15 & 21: 05: 06.639 & 33.177 & -115.635 & 5.3 \\ 2010 / 01 / 15 & 21: 18: 36.639 & 33.201 & -115.544 & 3.4 \\ 2010 / 01 / 15 & 21: 32: 14.639 & 33.185 & -115.608 & 2.0 \\ 2010 / 01 / 15 & 22: 06: 14.639 & 33.175 & -115.610 & 2.4 \\ 2010 / 01 / 15 & 22: 22: 14.639 & 33.176 & -115.609 & 2.1 \\ 2010 / 01 / 15 & 22: 46: 36.639 & 33.181 & -115.601 & 0.7 \\ 2010 / 01 / 15 & 23: 22: 57.639 & 33.182 & -115.612 & 2.2 \\ 2010 / 01 / 15 & 23: 33: 15.639 & 33.229 & -115.576 & 3.4 \\ 2010 / 01 / 15 & 23: 36: 40.639 & 33.184 & -115.604 & 0.1 \\ 2010 / 01 / 15 & 23: 42: 55.639 & 33.182 & -115.618 & 3.1 \\ 2010 / 01 / 15 & 23: 53: 45.639 & 33.181 & -115.603 & 2.0 \\ 2010 / 01 / 16 & 00: 05: 44.639 & 33.212 & -115.633 & 7.6 \\ 2010 / 01 / 16 & 00: 09: 30.639 & 33.188 & -115.609 & 2.3 \\ 2010 / 01 / 16 & 00: 15: 59.639 & 33.212 & -115.633 & 7.6 \\ 2010 / 01 / 16 & 00: 57: 36.639 & 33.188 & -115.609 & 2.3 \\ 2010 / 01 / 16 & 00: 59: 28.639 & 33.188 & -115.609 & 2.3 \\ 2010 / 01 / 16 & 01: 19: 56.639 & 33.188 & -115.609 & 2.3 \\ 2010 / 01 / 16 & 01: 24: 45.639 & 33.188 & -115.600 & 0.1 \\ 2010 / 01 / 16 & 01: 26: 19.639 & 33.182 & -115.612 & 2.2 \\ 2010 / 01 / 16 & 01: 30: 01.639 & 33.181 & -115.603 & 0.1 \\ 2010 / 01 / 16 & 01: 47: 08.639 & 33.186 & -115.604 & 2.0 \\ 2010 / 01 / 16 & 02: 36: 53.639 & 33.188 & -115.600 & 0.1 \\ 2010 / 01 / 16 & 03: 47: 07.639 & 33.176 & -115.604 & 0.0 \\ 2010 / 01 / 16 & 04: 05: 06.639 & 33.181 & -115.603 & 0.1 \\ 2010 / 01 / 16 & 04: 13: 10.639 & 33.181 & -115.603 & 1.4 \\ 2010 / 01 / 16 & 06: 11: 47.639 & 33.184 & -115.610 & 2.8 \\ 2010 / 01 / 16 & 06: 18: 09.639 & 33.193 & -115.583 & 5.9 \\ 2010 / 01 / 16 & 06: 32: 19.639 & 33.175 & -115.610 & 2.4 \\ 2010 / 01 / 16 & 06: 41: 31.639 & 33.184 & -115.608 & 0.3 \\ 2010 / 01 / 16 & 07: 22: 06.639 & 33.180 & -115.603 & 0.1 \\ 2010 / 01 / 16 & 08: 17: 45.639 & 33.173 & -115.609 & 0.1 \\ 2010 / 01 / 16 & 08: 19: 05.639 & 33.168 & -115.609 & 0.7 \\ 2010 / 01 / 16 & 09: 43: 38.639 & 33.188 & -115.609 & 2.3 \\ 2010 / 01 / 16 & 09: 51: 56.639 & 33.188 & -115.609 & 2.3 \\ 2010 / 01 / 16 & 10: 28: 07.639 & 33.212 & -115.633 & 7.6 \\ 2010 / 01 / 16 & 10: 56: 42.639 & 33.166 & -115.604 & 2.0 \\ 2010 / 01 / 16 & 12: 15: 47.639 & 33.188 & -115.598 & 2.3 \\ 201 / 161 / 16 & 12: 16: 27.639 & 33.181 & -115.665 & 4.2 \\ 20 & 12: 19: 21.639 & 33.181 & -115.665 & 4.2 \\ 2016 & 13: 11: 40.639 & 33.183 & -115.606 & 0.1\end{array}$




$\begin{array}{lllll}2010 / 01 / 16 & 13: 27: 01.639 & 33.181 & -115.603 & 2.0 \\ 2010 / 01 / 16 & 13: 44: 08.639 & 33.181 & -115.602 & 0.4 \\ 2010 / 01 / 16 & 13: 47: 49.639 & 33.188 & -115.598 & 2.3 \\ 2010 / 01 / 16 & 14: 39: 27.639 & 33.172 & -115.622 & 4.0 \\ 2010 / 01 / 16 & 14: 48: 29.639 & 33.181 & -115.602 & 0.4 \\ 2010 / 01 / 16 & 15: 09: 22.639 & 33.158 & -115.642 & 0.1 \\ 2010 / 01 / 16 & 15: 23: 30.639 & 33.181 & -115.601 & 0.7 \\ 2010 / 01 / 16 & 16: 47: 22.639 & 33.181 & -115.602 & 0.4 \\ 2010 / 01 / 16 & 17: 32: 01.639 & 33.184 & -115.608 & 0.3 \\ 2010 / 01 / 16 & 17: 32: 18.639 & 33.187 & -115.601 & 1.7 \\ 2010 / 01 / 16 & 17: 32: 47.639 & 33.187 & -115.601 & 1.7 \\ 2010 / 01 / 16 & 17: 51: 10.639 & 33.182 & -115.602 & 2.5 \\ 2010 / 01 / 16 & 18: 18: 32.639 & 33.229 & -115.645 & 5.7 \\ 2010 / 01 / 16 & 18: 32: 52.639 & 33.190 & -115.608 & 2.2 \\ 2010 / 01 / 16 & 19: 16: 13.639 & 33.176 & -115.604 & 0.5 \\ 2010 / 01 / 16 & 19: 21: 21.639 & 33.179 & -115.594 & 1.5 \\ 2010 / 01 / 16 & 20: 20: 14.639 & 33.117 & -115.613 & 7.9 \\ 2010 / 01 / 16 & 21: 10: 22.639 & 33.173 & -115.617 & 0.2 \\ 2010 / 01 / 16 & 21: 37: 39.639 & 33.208 & -115.631 & 5.0 \\ 2010 / 01 / 16 & 21: 57: 45.639 & 33.185 & -115.608 & 2.0 \\ 2010 / 01 / 17 & 02: 05: 26.639 & 33.176 & -115.618 & 3.7 \\ 2010 / 01 / 17 & 02: 23: 58.639 & 33.212 & -115.633 & 7.6 \\ 2010 / 01 / 17 & 03: 14: 09.639 & 33.172 & -115.622 & 4.0 \\ 2010 / 01 / 17 & 04: 59: 28.639 & 33.179 & -115.594 & 1.5 \\ 2010 / 01 / 17 & 04: 59: 39.639 & 33.179 & -115.594 & 1.5 \\ 2010 / 01 / 17 & 05: 28: 41.639 & 33.108 & -115.602 & 8.1 \\ 2010 / 01 / 17 & 07: 28: 13.639 & 33.176 & -115.608 & 0.4 \\ 2010 / 01 / 17 & 07: 29: 12.639 & 33.179 & -115.594 & 1.5 \\ 2010 / 01 / 17 & 07: 35: 48.639 & 33.176 & -115.608 & 0.4 \\ 2010 / 01 / 17 & 07: 36: 42.639 & 33.182 & -115.602 & 2.5 \\ 2010 / 01 / 17 & 08: 55: 36.639 & 33.218 & -115.639 & 3.0 \\ 2010 / 01 / 17 & 09: 03: 24.639 & 33.180 & -115.603 & 0.1 \\ 2010 / 01 / 17 & 09: 21: 06.639 & 33.176 & -115.604 & 0.5 \\ 2010 / 01 / 17 & 09: 30: 30.639 & 33.176 & -115.604 & 0.5 \\ 2010 / 01 / 17 & 11: 14: 38.639 & 33.187 & -115.604 & 3.2 \\ 2010 / 01 / 17 & 11: 38: 07.639 & 33.179 & -115.614 & 1.8 \\ 2010 / 01 / 17 & 12: 32: 52.639 & 33.187 & -115.604 & 3.2 \\ 2010 / 01 / 17 & 12: 45: 53.639 & 33.181 & -115.602 & 0.4 \\ 2010 / 01 / 17 & 12: 46: 34.639 & 33.214 & -115.620 & 5.3 \\ 2010 / 01 / 17 / 17 & 13: 05: 55.639 & 33.184 & -115.608 & 0.3 \\ 201 / 17 & 13: 34: 41.639 & 33.183 & -115.607 & 0.0 \\ 2017 & 13: 53: 24.639 & 33.176 & -115.626 & 3.7 \\ 2017 & 13: 54: 00.639 & 33.175 & -115.609 & 3.8 \\ 203: 36: 01.639 & 33.229 & -115.576 & 3.4\end{array}$




$\begin{array}{lllll}2010 / 01 / 17 & 15: 57: 33.639 & 33.179 & -115.597 & 0.1 \\ 2010 / 01 / 17 & 21: 04: 51.639 & 33.188 & -115.609 & 2.3 \\ 2010 / 01 / 17 & 21: 43: 49.639 & 33.187 & -115.604 & 3.2 \\ 2010 / 01 / 17 & 21: 59: 33.639 & 33.175 & -115.610 & 2.4 \\ 2010 / 01 / 17 & 22: 00: 05.639 & 33.176 & -115.618 & 3.7 \\ 2010 / 01 / 17 & 23: 23: 05.639 & 33.186 & -115.604 & 2.0 \\ 2010 / 01 / 18 & 00: 07: 40.639 & 33.184 & -115.610 & 2.8 \\ 2010 / 01 / 18 & 01: 03: 28.639 & 33.149 & -115.640 & 3.5 \\ 2010 / 01 / 18 & 01: 08: 53.639 & 33.193 & -115.632 & 3.5 \\ 2010 / 01 / 18 & 01: 21: 35.639 & 33.187 & -115.604 & 3.2 \\ 2010 / 01 / 18 & 02: 20: 31.639 & 33.188 & -115.600 & 0.1 \\ 2010 / 01 / 18 & 02: 23: 29.639 & 33.181 & -115.665 & 4.2 \\ 2010 / 01 / 18 & 02: 33: 43.639 & 33.157 & -115.669 & 0.6 \\ 2010 / 01 / 18 & 04: 37: 01.639 & 33.176 & -115.609 & 2.1 \\ 2010 / 01 / 18 & 05: 10: 59.639 & 33.188 & -115.609 & 2.3 \\ 2010 / 01 / 18 & 05: 42: 01.639 & 33.188 & -115.609 & 2.3 \\ 2010 / 01 / 18 & 06: 00: 51.639 & 33.188 & -115.609 & 2.3 \\ 2010 / 01 / 18 & 06: 49: 01.639 & 33.182 & -115.603 & 0.1 \\ 2010 / 01 / 18 & 06: 54: 47.639 & 33.182 & -115.603 & 0.1 \\ 2010 / 01 / 18 & 07: 26: 06.639 & 33.179 & -115.597 & 0.1 \\ 2010 / 01 / 18 & 08: 19: 38.639 & 33.181 & -115.602 & 0.4 \\ 2010 / 01 / 18 & 09: 15: 25.639 & 33.187 & -115.604 & 3.2 \\ 2010 / 01 / 18 & 09: 17: 06.639 & 33.187 & -115.604 & 3.2 \\ 2010 / 01 / 18 & 10: 17: 41.639 & 33.188 & -115.609 & 2.3 \\ 2010 / 01 / 18 & 11: 15: 33.639 & 33.174 & -115.603 & 0.1 \\ 2010 / 01 / 18 & 11: 23: 20.639 & 33.187 & -115.604 & 3.2 \\ 2010 / 01 / 18 & 11: 49: 56.639 & 33.149 & -115.640 & 3.5 \\ 2010 / 01 / 18 & 12: 09: 35.639 & 33.172 & -115.611 & 3.7 \\ 2010 / 01 / 18 & 12: 30: 24.639 & 33.187 & -115.604 & 3.2 \\ 2010 / 01 / 18 & 13: 26: 32.639 & 33.176 & -115.608 & 0.4 \\ 2010 / 01 / 18 & 19: 04: 08.639 & 33.108 & -115.602 & 8.1 \\ 2010 / 01 / 18 & 19: 06: 20.639 & 33.159 & -115.641 & 0.9 \\ 2010 / 01 / 19 & 02: 27: 12.639 & 33.175 & -115.606 & 4.2 \\ 2010 / 01 / 19 & 02: 28: 00.639 & 33.205 & -115.577 & 0.0 \\ 2010 / 01 / 19 & 02: 34: 48.639 & 33.212 & -115.633 & 7.6 \\ 2010 / 01 / 19 & 02: 36: 28.639 & 33.212 & -115.633 & 7.6 \\ 2010 / 01 / 19 & 03: 58: 57.639 & 33.176 & -115.604 & 0.0 \\ 2010 / 01 / 19 & 04: 04: 30.639 & 33.174 & -115.603 & 0.1 \\ 2010 / 01 / 19 & 05: 16: 00.639 & 33.192 & -115.572 & 1.6 \\ 2010 / 01 / 19 / 01 / 19 & 06: 55: 25.639 & 33.176 & -115.604 & 0.5 \\ 20101 / 19 & 06: 56: 25.639 & 33.177 & -115.606 & 0.1 \\ 26: 59: 03.639 & 33.170 & -115.600 & 1.1 \\ 201 / 19 & 09: 33: 56.639 & 33.186 & -115.604 & 2.0\end{array}$




$\begin{array}{lllll}2010 / 01 / 19 & 09: 34: 11.639 & 33.173 & -115.650 & 1.8 \\ 2010 / 01 / 19 & 09: 49: 17.639 & 33.234 & -115.604 & 4.8 \\ 2010 / 01 / 19 & 09: 51: 01.639 & 33.181 & -115.601 & 0.7 \\ 2010 / 01 / 19 & 10: 18: 28.639 & 33.180 & -115.600 & 0.1 \\ 2010 / 01 / 19 & 11: 00: 41.639 & 33.176 & -115.608 & 0.4 \\ 2010 / 01 / 19 & 11: 03: 17.639 & 33.175 & -115.606 & 4.2 \\ 2010 / 01 / 19 & 11: 05: 09.639 & 33.182 & -115.602 & 2.5 \\ 2010 / 01 / 19 & 11: 06: 13.639 & 33.182 & -115.602 & 2.5 \\ 2010 / 01 / 19 & 11: 07: 01.639 & 33.182 & -115.602 & 2.5 \\ 2010 / 01 / 19 & 11: 24: 19.639 & 33.182 & -115.602 & 2.5 \\ 2010 / 01 / 19 & 11: 26: 35.639 & 33.175 & -115.606 & 4.2 \\ 2010 / 01 / 19 & 11: 26: 58.639 & 33.175 & -115.606 & 4.2 \\ 2010 / 01 / 19 & 11: 27: 42.639 & 33.176 & -115.604 & 0.5 \\ 2010 / 01 / 19 & 11: 28: 18.639 & 33.177 & -115.606 & 0.1 \\ 2010 / 01 / 19 & 11: 37: 51.639 & 33.179 & -115.594 & 1.5 \\ 2010 / 01 / 19 & 12: 35: 41.639 & 33.229 & -115.645 & 5.7 \\ 2010 / 01 / 19 & 13: 15: 49.639 & 33.176 & -115.604 & 0.0 \\ 2010 / 01 / 19 & 13: 59: 46.639 & 33.175 & -115.606 & 4.2 \\ 2010 / 01 / 19 & 14: 02: 09.639 & 33.175 & -115.606 & 4.2 \\ 2010 / 01 / 19 & 14: 02: 33.639 & 33.176 & -115.609 & 3.8 \\ 2010 / 01 / 19 & 14: 03: 26.639 & 33.175 & -115.606 & 4.2 \\ 2010 / 01 / 19 & 14: 09: 20.639 & 33.183 & -115.617 & 4.3 \\ 2010 / 01 / 19 & 14: 34: 54.639 & 33.176 & -115.609 & 3.8 \\ 2010 / 01 / 19 & 14: 43: 40.639 & 33.179 & -115.594 & 1.5 \\ 2010 / 01 / 19 & 16: 37: 01.639 & 33.177 & -115.614 & 1.2 \\ 2010 / 01 / 19 & 16: 43: 13.639 & 33.184 & -115.601 & 3.5 \\ 2010 / 01 / 19 & 16: 46: 46.639 & 33.187 & -115.622 & 4.0 \\ 2010 / 01 / 19 & 18: 28: 46.639 & 33.184 & -115.610 & 2.8 \\ 2010 / 01 / 19 & 18: 38: 54.639 & 33.184 & -115.610 & 2.8 \\ 2010 / 01 / 19 & 20: 15: 10.639 & 33.108 & -115.602 & 8.1 \\ 2010 / 01 / 19 & 20: 17: 41.639 & 33.175 & -115.603 & 0.1 \\ 2010 / 01 / 19 & 23: 50: 37.639 & 33.184 & -115.601 & 3.5 \\ 2010 / 01 / 20 & 03: 04: 16.639 & 33.199 & -115.570 & 0.2 \\ 2010 / 01 / 20 & 04: 43: 57.639 & 33.196 & -115.587 & 5.3 \\ 2010 / 01 / 20 & 05: 01: 29.639 & 33.176 & -115.604 & 0.0 \\ 2010 / 01 / 20 & 06: 18: 05.639 & 33.157 & -115.641 & 2.2 \\ 2010 / 01 / 20 & 07: 00: 46.639 & 33.188 & -115.573 & 0.2 \\ 2010 / 01 / 20 & 08: 41: 11.639 & 33.108 & -115.602 & 8.1 \\ 2010 / 01 / 20 & 10: 18: 34.639 & 33.184 & -115.608 & 0.3 \\ 2010 / 01 / 20 & 11: 05: 00.639 & 33.181 & -115.608 & 1.0 \\ 201 / 20 & 15: 17: 36.639 & 33.212 & -115.633 & 7.6 \\ 201 / 20 & 17: 33: 42.639 & 33.176 & -115.621 & 2.7 \\ 20 & 17: 33: 55.639 & 33.181 & -115.608 & 0.4 \\ 20 & 19: 38: 07.639 & 33.174 & -115.603 & 0.1 \\ 20: 04: 37.639 & 33.182 & -115.612 & 2.2\end{array}$




$\begin{array}{lllll}2010 / 01 / 20 & 20: 04: 53.639 & 33.182 & -115.612 & 2.2 \\ 2010 / 01 / 20 & 23: 27: 05.639 & 33.108 & -115.602 & 8.1 \\ 2010 / 01 / 20 & 23: 42: 03.639 & 33.108 & -115.602 & 8.1 \\ 2010 / 01 / 21 & 01: 36: 05.639 & 33.108 & -115.602 & 8.1 \\ 2010 / 01 / 21 & 04: 47: 06.639 & 33.176 & -115.608 & 0.4 \\ 2010 / 01 / 21 & 05: 32: 34.639 & 33.192 & -115.626 & 7.7 \\ 2010 / 01 / 21 & 08: 37: 26.639 & 33.228 & -115.626 & 3.7 \\ 2010 / 01 / 21 & 08: 51: 27.639 & 33.157 & -115.680 & 0.2 \\ 2010 / 01 / 21 & 09: 56: 10.639 & 33.108 & -115.602 & 8.1 \\ 2010 / 01 / 21 & 10: 19: 18.639 & 33.108 & -115.602 & 8.1 \\ 2010 / 01 / 21 & 10: 24: 18.639 & 33.158 & -115.642 & 0.1 \\ 2010 / 01 / 21 & 11: 28: 59.639 & 33.179 & -115.594 & 1.5 \\ 2010 / 01 / 21 & 14: 00: 38.639 & 33.176 & -115.609 & 2.1 \\ 2010 / 01 / 21 & 14: 00: 52.639 & 33.140 & -115.636 & 7.3 \\ 2010 / 01 / 21 & 14: 14: 37.639 & 33.158 & -115.644 & 0.1 \\ 2010 / 01 / 21 & 16: 01: 49.639 & 33.185 & -115.608 & 2.0 \\ 2010 / 01 / 21 & 16: 03: 25.639 & 33.185 & -115.608 & 2.0 \\ 2010 / 01 / 21 & 16: 54: 51.639 & 33.190 & -115.608 & 2.2 \\ 2010 / 01 / 21 & 16: 57: 55.639 & 33.212 & -115.633 & 7.6 \\ 2010 / 01 / 21 & 17: 32: 07.639 & 33.194 & -115.583 & 3.0 \\ 2010 / 01 / 21 & 18: 20: 40.639 & 33.175 & -115.600 & 1.1 \\ 2010 / 01 / 21 & 19: 16: 33.639 & 33.172 & -115.622 & 4.0 \\ 2010 / 01 / 21 & 19: 19: 35.639 & 33.172 & -115.622 & 4.0 \\ 2010 / 01 / 21 & 20: 17: 38.639 & 33.192 & -115.626 & 7.7 \\ 2010 / 01 / 21 & 20: 41: 09.639 & 33.108 & -115.602 & 8.1 \\ 2010 / 01 / 21 & 20: 44: 21.639 & 33.118 & -115.610 & 9.1 \\ 2010 / 01 / 21 & 20: 45: 28.639 & 33.149 & -115.640 & 3.5 \\ 2010 / 01 / 21 & 20: 46: 32.639 & 33.117 & -115.613 & 7.9 \\ 2010 / 01 / 21 & 20: 47: 48.639 & 33.159 & -115.641 & 0.9 \\ 2010 / 01 / 21 & 20: 49: 47.639 & 33.181 & -115.612 & 1.1 \\ 2010 / 01 / 21 & 20: 52: 58.639 & 33.182 & -115.606 & 1.7 \\ 2010 / 01 / 21 & 20: 53: 54.639 & 33.158 & -115.638 & 0.4 \\ 2010 / 01 / 21 & 20: 55: 01.639 & 33.161 & -115.651 & 0.7 \\ 2010 / 01 / 21 & 21: 16: 00.639 & 33.117 & -115.613 & 7.9 \\ 2010 / 01 / 21 & 21: 31: 10.639 & 33.181 & -115.659 & 6.0 \\ 2010 / 01 / 21 & 21: 46: 01.639 & 33.140 & -115.636 & 7.3 \\ 2010 / 01 / 21 & 22: 13: 15.639 & 33.161 & -115.651 & 0.7 \\ 2010 / 01 / 21 & 22: 53: 43.639 & 33.158 & -115.650 & 0.0 \\ 2010 / 01 / 22 & 00: 39: 16.639 & 33.203 & -115.578 & 2.2 \\ 2010 / 01 / 22 & 01: 05: 41.639 & 33.179 & -115.602 & 0.6 \\ 2010 / 01 / 22 & 01: 50: 09.639 & 33.174 & -115.603 & 0.1 \\ 2010 / 01 / 22 & 03: 18: 59.639 & 33.187 & -115.608 & 0.2 \\ 2010 / 01 / 22 & 04: 00: 10.639 & 33.161 & -115.644 & 1.0 \\ 2010 / 01 / 22 & 04: 05: 51.639 & 33.161 & -115.644 & 1.0 \\ 201 / 22 & 04: 25: 27.639 & 33.161 & -115.644 & 1.0\end{array}$




$\begin{array}{lllll}2010 / 01 / 22 & 05: 40: 04.639 & 33.231 & -115.655 & 4.8 \\ 2010 / 01 / 22 & 06: 54: 39.639 & 33.117 & -115.613 & 7.9 \\ 2010 / 01 / 22 & 07: 42: 29.639 & 33.188 & -115.609 & 2.3 \\ 2010 / 01 / 22 & 07: 44: 33.639 & 33.161 & -115.644 & 1.0 \\ 2010 / 01 / 22 & 08: 16: 11.639 & 33.161 & -115.644 & 1.0 \\ 2010 / 01 / 22 & 09: 24: 48.639 & 33.180 & -115.600 & 0.1 \\ 2010 / 01 / 22 & 09: 41: 54.639 & 33.161 & -115.644 & 1.0 \\ 2010 / 01 / 22 & 10: 02: 45.639 & 33.180 & -115.600 & 0.1 \\ 2010 / 01 / 22 & 10: 04: 52.639 & 33.108 & -115.602 & 8.1 \\ 2010 / 01 / 22 & 10: 12: 40.639 & 33.161 & -115.644 & 0.3 \\ 2010 / 01 / 22 & 10: 19: 34.639 & 33.108 & -115.602 & 8.1 \\ 2010 / 01 / 22 & 10: 30: 52.639 & 33.163 & -115.637 & 0.1 \\ 2010 / 01 / 22 & 11: 00: 46.639 & 33.208 & -115.631 & 5.0 \\ 2010 / 01 / 22 & 11: 06: 33.639 & 33.165 & -115.649 & 1.4 \\ 2010 / 01 / 22 & 11: 08: 04.639 & 33.165 & -115.649 & 1.4 \\ 2010 / 01 / 22 & 11: 08: 55.639 & 33.165 & -115.649 & 1.4 \\ 2010 / 01 / 22 & 11: 11: 01.639 & 33.165 & -115.649 & 1.4 \\ 2010 / 01 / 22 & 11: 14: 47.639 & 33.161 & -115.644 & 0.3 \\ 2010 / 01 / 22 & 11: 15: 45.639 & 33.165 & -115.649 & 1.4 \\ 2010 / 01 / 22 & 11: 29: 14.639 & 33.179 & -115.597 & 0.1 \\ 2010 / 01 / 22 & 11: 31: 51.639 & 33.183 & -115.599 & 0.1 \\ 2010 / 01 / 22 & 12: 45: 34.639 & 33.184 & -115.606 & 2.9 \\ 2010 / 01 / 22 & 13: 16: 40.639 & 33.172 & -115.622 & 4.0 \\ 2010 / 01 / 22 & 13: 21: 18.639 & 33.172 & -115.611 & 3.7 \\ 2010 / 01 / 22 & 14: 33: 29.639 & 33.231 & -115.655 & 4.8 \\ 2010 / 01 / 22 & 16: 34: 04.639 & 33.145 & -115.634 & 4.8 \\ 2010 / 01 / 22 & 17: 08: 14.639 & 33.145 & -115.634 & 4.8 \\ 2010 / 01 / 22 & 17: 09: 29.639 & 33.148 & -115.633 & 4.3 \\ 2010 / 01 / 22 & 17: 12: 32.639 & 33.148 & -115.633 & 4.3 \\ 2010 / 01 / 22 & 17: 13: 42.639 & 33.179 & -115.597 & 0.1 \\ 2010 / 01 / 23 & 03: 24: 18.639 & 33.118 & -115.610 & 9.1 \\ 2010 / 01 / 23 & 03: 28: 12.639 & 33.130 & -115.634 & 0.0 \\ 2010 / 01 / 23 & 07: 21: 14.639 & 33.181 & -115.659 & 6.0 \\ 2010 / 01 / 23 & 07: 38: 31.639 & 33.192 & -115.626 & 7.7 \\ 2010 / 01 / 23 & 08: 42: 37.639 & 33.199 & -115.571 & 3.3 \\ 2010 / 01 / 23 & 08: 45: 02.639 & 33.161 & -115.644 & 1.0 \\ 2010 / 01 / 23 & 09: 19: 50.639 & 33.176 & -115.609 & 2.1 \\ 2010 / 01 / 23 & 09: 26: 01.639 & 33.158 & -115.638 & 0.4 \\ 2010 / 01 / 23 & 13: 06: 35.639 & 33.157 & -115.648 & 0.1 \\ 2010 / 01 / 23 & 13: 08: 29.639 & 33.135 & -115.644 & 6.9 \\ 2010 / 01 / 23 & 13: 09: 08.639 & 33.159 & -115.640 & 1.5 \\ 2010 / 01 / 23 & 13: 09: 55.639 & 33.130 & -115.634 & 0.0 \\ 2010 / 01 / 23 & 13: 10: 50.639 & 33.159 & -115.640 & 1.5 \\ 2010 / 01 / 23 & 13: 11: 24.639 & 33.159 & -115.640 & 1.5 \\ & 13: 12: 02.639 & 33.159 & -115.640 & 1.5 \\ 23: 24.639 & 33.159 & -115.640 & 1.5\end{array}$




$\begin{array}{lllll}2010 / 01 / 23 & 13: 13: 03.639 & 33.159 & -115.640 & 1.5 \\ 2010 / 01 / 23 & 13: 13: 51.639 & 33.108 & -115.602 & 8.1 \\ 2010 / 01 / 23 & 13: 14: 02.639 & 33.158 & -115.638 & 0.4 \\ 2010 / 01 / 23 & 13: 14: 25.639 & 33.135 & -115.644 & 6.9 \\ 2010 / 01 / 23 & 13: 15: 24.639 & 33.159 & -115.640 & 1.5 \\ 2010 / 01 / 23 & 13: 15: 38.639 & 33.135 & -115.644 & 6.9 \\ 2010 / 01 / 23 & 13: 16: 17.639 & 33.130 & -115.634 & 0.0 \\ 2010 / 01 / 23 & 13: 17: 17.639 & 33.164 & -115.640 & 3.4 \\ 2010 / 01 / 23 & 13: 18: 00.639 & 33.164 & -115.640 & 3.4 \\ 2010 / 01 / 23 & 13: 18: 19.639 & 33.164 & -115.640 & 3.4 \\ 2010 / 01 / 23 & 13: 19: 02.639 & 33.158 & -115.644 & 0.1 \\ 2010 / 01 / 23 & 13: 19: 52.639 & 33.164 & -115.640 & 3.4 \\ 2010 / 01 / 23 & 13: 20: 04.639 & 33.164 & -115.640 & 3.4 \\ 2010 / 01 / 23 & 13: 20: 34.639 & 33.161 & -115.640 & 0.1 \\ 2010 / 01 / 23 & 13: 21: 24.639 & 33.117 & -115.613 & 7.9 \\ 2010 / 01 / 23 & 13: 21: 45.639 & 33.130 & -115.634 & 0.0 \\ 2010 / 01 / 23 & 13: 22: 28.639 & 33.164 & -115.640 & 3.4 \\ 2010 / 01 / 23 & 13: 23: 11.639 & 33.158 & -115.644 & 0.1 \\ 2010 / 01 / 23 & 13: 23: 39.639 & 33.108 & -115.602 & 8.1 \\ 2010 / 01 / 23 & 13: 24: 18.639 & 33.158 & -115.644 & 0.1 \\ 2010 / 01 / 23 & 13: 24: 59.639 & 33.164 & -115.640 & 3.4 \\ 2010 / 01 / 23 & 13: 25: 27.639 & 33.164 & -115.640 & 3.4 \\ 2010 / 01 / 23 & 13: 26: 05.639 & 33.157 & -115.641 & 2.2 \\ 2010 / 01 / 23 & 13: 26: 31.639 & 33.159 & -115.640 & 1.5 \\ 2010 / 01 / 23 & 13: 27: 26.639 & 33.159 & -115.640 & 1.5 \\ 2010 / 01 / 23 & 13: 28: 29.639 & 33.161 & -115.640 & 0.1 \\ 2010 / 01 / 23 & 13: 28: 55.639 & 33.158 & -115.644 & 0.1 \\ 2010 / 01 / 23 & 13: 29: 27.639 & 33.158 & -115.638 & 0.4 \\ 2010 / 01 / 23 & 13: 30: 54.639 & 33.164 & -115.640 & 3.4 \\ 2010 / 01 / 23 & 13: 31: 43.639 & 33.160 & -115.642 & 1.5 \\ 2010 / 01 / 23 & 13: 32: 43.639 & 33.108 & -115.602 & 8.1 \\ 2010 / 01 / 23 & 13: 33: 15.639 & 33.160 & -115.642 & 1.5 \\ 2010 / 01 / 23 & 13: 33: 55.639 & 33.164 & -115.640 & 3.4 \\ 2010 / 01 / 23 & 13: 35: 15.639 & 33.164 & -115.640 & 3.4 \\ 2010 / 01 / 23 & 13: 35: 36.639 & 33.158 & -115.638 & 0.4 \\ 2010 / 01 / 23 & 13: 38: 10.639 & 33.159 & -115.640 & 1.5 \\ 2010 / 01 / 23 & 13: 38: 53.639 & 33.135 & -115.644 & 6.9 \\ 2010 / 01 / 23 & 13: 40: 35.639 & 33.108 & -115.602 & 8.1 \\ 2010 / 01 / 23 & 13: 41: 26.639 & 33.164 & -115.640 & 3.4 \\ 2010 / 01 / 23 & 13: 43: 27.639 & 33.149 & -115.640 & 3.5 \\ 2010 / 01 / 23 & 13: 44: 09.639 & 33.161 & -115.640 & 0.1 \\ 201 / 23 & 13: 44: 35.639 & 33.108 & -115.602 & 8.1 \\ 23 & 13: 46: 22.639 & 33.159 & -115.640 & 1.5 \\ 23 & 13: 49: 24.639 & 33.231 & -115.655 & 4.8\end{array}$




$\begin{array}{lllll}2010 / 01 / 23 & 13: 51: 10.639 & 33.161 & -115.640 & 0.1 \\ 2010 / 01 / 23 & 14: 02: 09.639 & 33.161 & -115.640 & 0.1 \\ 2010 / 01 / 23 & 14: 05: 18.639 & 33.164 & -115.640 & 3.4 \\ 2010 / 01 / 23 & 14: 06: 36.639 & 33.177 & -115.606 & 0.1 \\ 2010 / 01 / 23 & 14: 10: 11.639 & 33.108 & -115.602 & 8.1 \\ 2010 / 01 / 23 & 14: 12: 06.639 & 33.157 & -115.641 & 2.2 \\ 2010 / 01 / 23 & 14: 13: 57.639 & 33.164 & -115.640 & 3.4 \\ 2010 / 01 / 23 & 14: 17: 54.639 & 33.164 & -115.640 & 3.4 \\ 2010 / 01 / 23 & 14: 22: 05.639 & 33.149 & -115.640 & 3.5 \\ 2010 / 01 / 23 & 14: 24: 28.639 & 33.158 & -115.638 & 0.4 \\ 2010 / 01 / 23 & 14: 25: 47.639 & 33.149 & -115.640 & 3.5 \\ 2010 / 01 / 23 & 14: 26: 24.639 & 33.161 & -115.644 & 1.0 \\ 2010 / 01 / 23 & 14: 27: 49.639 & 33.135 & -115.644 & 6.9 \\ 2010 / 01 / 23 & 14: 28: 32.639 & 33.108 & -115.602 & 8.1 \\ 2010 / 01 / 23 & 14: 30: 07.639 & 33.108 & -115.602 & 8.1 \\ 2010 / 01 / 23 & 14: 30: 30.639 & 33.189 & -115.635 & 3.2 \\ 2010 / 01 / 23 & 14: 33: 29.639 & 33.164 & -115.632 & 2.6 \\ 2010 / 01 / 23 & 14: 38: 29.639 & 33.164 & -115.640 & 3.4 \\ 2010 / 01 / 23 & 14: 59: 37.639 & 33.186 & -115.612 & 0.6 \\ 2010 / 01 / 23 & 15: 02: 36.639 & 33.172 & -115.611 & 3.7 \\ 2010 / 01 / 23 & 15: 03: 12.639 & 33.172 & -115.611 & 3.7 \\ 2010 / 01 / 23 & 15: 04: 02.639 & 33.181 & -115.639 & 3.7 \\ 2010 / 01 / 23 & 15: 09: 20.639 & 33.108 & -115.602 & 8.1 \\ 2010 / 01 / 23 & 15: 11: 53.639 & 33.157 & -115.680 & 0.2 \\ 2010 / 01 / 23 & 15: 12: 23.639 & 33.161 & -115.640 & 0.1 \\ 2010 / 01 / 23 & 15: 12: 56.639 & 33.170 & -115.626 & 0.6 \\ 2010 / 01 / 23 & 15: 20: 41.639 & 33.157 & -115.641 & 2.2 \\ 2010 / 01 / 23 & 15: 27: 25.639 & 33.108 & -115.602 & 8.1 \\ 2010 / 01 / 23 & 15: 30: 13.639 & 33.149 & -115.640 & 3.5 \\ 2010 / 01 / 23 & 15: 32: 00.639 & 33.158 & -115.642 & 0.1 \\ 2010 / 01 / 23 & 15: 33: 52.639 & 33.158 & -115.644 & 0.1 \\ 2010 / 01 / 23 & 15: 34: 36.639 & 33.135 & -115.644 & 6.9 \\ 2010 / 01 / 23 & 15: 38: 13.639 & 33.166 & -115.642 & 2.3 \\ 2010 / 01 / 23 & 15: 40: 15.639 & 33.135 & -115.644 & 6.9 \\ 2010 / 01 / 23 & 16: 14: 07.639 & 33.166 & -115.642 & 2.3 \\ 2010 / 01 / 23 & 17: 24: 33.639 & 33.165 & -115.623 & 7.0 \\ 2010 / 01 / 23 & 17: 32: 34.639 & 33.158 & -115.644 & 0.1 \\ 2010 / 01 / 23 & 17: 49: 48.639 & 33.161 & -115.644 & 0.3 \\ 2010 / 01 / 23 & 19: 12: 13.639 & 33.140 & -115.636 & 7.3 \\ 2010 / 01 / 23 & 20: 21: 51.639 & 33.190 & -115.574 & 3.1 \\ 2010 / 01 / 23 & 20: 41: 01.639 & 33.229 & -115.576 & 3.4 \\ 2010 / 01 / 23 & 20: 51: 08.639 & 33.185 & -115.608 & 2.2 \\ 201 / 23 & 21: 42: 09.639 & 33.190 & -115.608 & 2.2 \\ 22: 25: 10.639 & 33.179 & -115.621 & 4.8 \\ 23 & 01: 18: 12.639 & 33.158 & -115.644 & 0.1\end{array}$




$\begin{array}{lllll}2010 / 01 / 24 & 01: 55: 12.639 & 33.158 & -115.642 & 0.1 \\ 2010 / 01 / 24 & 02: 00: 09.639 & 33.165 & -115.643 & 1.7 \\ 2010 / 01 / 24 & 02: 04: 13.639 & 33.158 & -115.644 & 0.1 \\ 2010 / 01 / 24 & 02: 33: 14.639 & 33.183 & -115.617 & 4.3 \\ 2010 / 01 / 24 & 02: 33: 59.639 & 33.184 & -115.604 & 0.1 \\ 2010 / 01 / 24 & 03: 19: 31.639 & 33.180 & -115.600 & 0.1 \\ 2010 / 01 / 24 & 08: 06: 25.639 & 33.172 & -115.611 & 3.7 \\ 2010 / 01 / 24 & 08: 09: 38.639 & 33.118 & -115.610 & 9.1 \\ 2010 / 01 / 24 & 12: 23: 07.639 & 33.184 & -115.610 & 2.8 \\ 2010 / 01 / 24 & 12: 23: 32.639 & 33.181 & -115.602 & 0.4 \\ 2010 / 01 / 24 & 20: 12: 52.639 & 33.163 & -115.637 & 0.1 \\ 2010 / 01 / 24 & 20: 17: 20.639 & 33.163 & -115.637 & 0.1 \\ 2010 / 01 / 24 & 20: 58: 59.639 & 33.108 & -115.602 & 8.1 \\ 2010 / 01 / 24 & 23: 53: 52.639 & 33.231 & -115.657 & 2.0 \\ 2010 / 01 / 24 & 23: 54: 44.639 & 33.248 & -115.633 & 2.9 \\ 2010 / 01 / 25 & 02: 47: 13.639 & 33.184 & -115.604 & 0.1 \\ 2010 / 01 / 25 & 04: 30: 19.639 & 33.174 & -115.603 & 0.1 \\ 2010 / 01 / 25 & 06: 03: 13.639 & 33.181 & -115.602 & 0.4 \\ 2010 / 01 / 25 & 06: 03: 48.639 & 33.181 & -115.602 & 0.4 \\ 2010 / 01 / 25 & 12: 36: 29.639 & 33.212 & -115.633 & 7.6 \\ 2010 / 01 / 25 & 13: 30: 33.639 & 33.184 & -115.604 & 0.1 \\ 2010 / 01 / 25 & 16: 29: 03.639 & 33.181 & -115.659 & 6.0 \\ 2010 / 01 / 25 & 18: 47: 11.639 & 33.181 & -115.659 & 6.0 \\ 2010 / 01 / 26 & 02: 05: 50.639 & 33.194 & -115.567 & 3.1 \\ 2010 / 01 / 26 & 05: 27: 01.639 & 33.187 & -115.604 & 3.2 \\ 2010 / 01 / 26 & 07: 11: 08.639 & 33.176 & -115.608 & 0.4 \\ 2010 / 01 / 26 & 07: 37: 32.639 & 33.189 & -115.635 & 3.2 \\ 2010 / 01 / 26 & 08: 21: 04.639 & 33.142 & -115.628 & 10.3 \\ 2010 / 01 / 26 & 09: 31: 57.639 & 33.182 & -115.602 & 2.5 \\ 2010 / 01 / 26 & 09: 32: 10.639 & 33.176 & -115.604 & 0.5 \\ 2010 / 01 / 26 & 09: 33: 11.639 & 33.179 & -115.594 & 1.5 \\ 2010 / 01 / 26 & 09: 36: 25.639 & 33.176 & -115.604 & 0.5 \\ 2010 / 01 / 26 & 12: 02: 18.639 & 33.108 & -115.602 & 8.1 \\ 2010 / 01 / 26 & 12: 24: 20.639 & 33.139 & -115.602 & 7.0 \\ 2010 / 01 / 26 & 13: 01: 41.639 & 33.185 & -115.608 & 2.0 \\ 2010 / 01 / 26 & 13: 16: 03.639 & 33.187 & -115.604 & 3.2 \\ 2010 / 01 / 26 & 14: 02: 17.639 & 33.187 & -115.604 & 3.2 \\ 2010 / 01 / 26 & 14: 55: 44.639 & 33.188 & -115.609 & 2.3 \\ 2010 / 01 / 26 & 16: 02: 21.639 & 33.108 & -115.602 & 8.1 \\ 2010 / 01 / 26 & 16: 11: 59.639 & 33.218 & -115.639 & 3.0 \\ 2010 / 01 / 26 & 16: 36: 10.639 & 33.174 & -115.603 & 0.1 \\ 2010 / 01 / 26 & 18: 14: 58.639 & 33.174 & -115.603 & 0.1 \\ 2010 / 01 / 26 & 19: 38: 38.639 & 33.176 & -115.608 & 0.4 \\ 2010 / 01 / 26 & 19: 39: 52.639 & 33.176 & -115.604 & 0.5 \\ 2010 / 01 / 26 & 19: 47: 44.639 & 33.176 & -115.608 & 0.4\end{array}$




$\begin{array}{lllll}2010 / 01 / 26 & 19: 49: 15.639 & 33.176 & -115.604 & 0.5 \\ 2010 / 01 / 26 & 19: 49: 42.639 & 33.176 & -115.604 & 0.5 \\ 2010 / 01 / 26 & 20: 21: 35.639 & 33.181 & -115.665 & 4.2 \\ 2010 / 01 / 27 & 02: 05: 46.639 & 33.183 & -115.617 & 4.3 \\ 2010 / 01 / 27 & 02: 16: 16.639 & 33.108 & -115.602 & 8.1 \\ 2010 / 01 / 27 & 02: 17: 32.639 & 33.108 & -115.602 & 8.1 \\ 2010 / 01 / 27 & 02: 17: 58.639 & 33.108 & -115.602 & 8.1 \\ 2010 / 01 / 27 & 03: 38: 41.639 & 33.174 & -115.603 & 0.1 \\ 2010 / 01 / 27 & 08: 38: 30.639 & 33.161 & -115.640 & 0.1 \\ 2010 / 01 / 27 & 08: 50: 28.639 & 33.174 & -115.603 & 0.1 \\ 2010 / 01 / 27 & 10: 40: 02.639 & 33.179 & -115.594 & 1.5 \\ 2010 / 01 / 27 & 10: 42: 05.639 & 33.179 & -115.594 & 1.5 \\ 2010 / 01 / 27 & 10: 43: 07.639 & 33.179 & -115.594 & 1.5 \\ 2010 / 01 / 27 & 10: 43: 35.639 & 33.170 & -115.600 & 1.1 \\ 2010 / 01 / 27 & 11: 51: 56.639 & 33.181 & -115.665 & 4.2 \\ 2010 / 01 / 27 & 19: 03: 09.639 & 33.231 & -115.657 & 2.0 \\ 2010 / 01 / 27 & 19: 22: 23.639 & 33.174 & -115.603 & 0.1 \\ 2010 / 01 / 28 & 02: 22: 56.639 & 33.100 & -115.590 & 14.6 \\ 2010 / 01 / 28 & 03: 27: 31.639 & 33.180 & -115.600 & 0.1 \\ 2010 / 01 / 28 & 05: 37: 40.639 & 33.208 & -115.631 & 5.0 \\ 2010 / 01 / 28 & 06: 58: 15.639 & 33.176 & -115.604 & 0.0 \\ 2010 / 01 / 28 & 09: 37: 04.639 & 33.192 & -115.579 & 4.3 \\ 2010 / 01 / 28 & 09: 40: 41.639 & 33.179 & -115.614 & 1.8 \\ 2010 / 01 / 28 & 09: 41: 19.639 & 33.190 & -115.608 & 2.2 \\ 2010 / 01 / 28 & 13: 43: 40.639 & 33.183 & -115.617 & 4.3 \\ 2010 / 01 / 28 & 13: 45: 33.639 & 33.174 & -115.603 & 0.1 \\ 2010 / 01 / 28 & 18: 17: 57.639 & 33.198 & -115.571 & 0.2 \\ 2010 / 01 / 28 & 18: 23: 58.639 & 33.198 & -115.571 & 0.2 \\ 2010 / 01 / 28 & 18: 24: 16.639 & 33.198 & -115.571 & 0.2 \\ 2010 / 01 / 28 & 18: 25: 02.639 & 33.198 & -115.571 & 0.2 \\ 2010 / 01 / 28 & 18: 25: 49.639 & 33.198 & -115.571 & 0.2 \\ 2010 / 01 / 28 & 18: 26: 56.639 & 33.196 & -115.573 & 0.1 \\ 2010 / 01 / 28 & 18: 30: 24.639 & 33.198 & -115.571 & 0.2 \\ 2010 / 01 / 28 & 18: 32: 12.639 & 33.198 & -115.571 & 0.2 \\ 2010 / 01 / 28 & 18: 33: 11.639 & 33.196 & -115.573 & 0.1 \\ 2010 / 01 / 28 & 18: 39: 03.639 & 33.192 & -115.579 & 4.3 \\ 2010 / 01 / 28 & 18: 42: 19.639 & 33.192 & -115.579 & 4.3 \\ 2010 / 01 / 28 & 18: 46: 04.639 & 33.198 & -115.571 & 0.2 \\ 2010 / 01 / 28 & 18: 48: 46.639 & 33.198 & -115.571 & 0.2 \\ 2010 / 01 / 28 & 19: 42: 13.639 & 33.192 & -115.579 & 4.3 \\ 2010 / 01 / 28 & 20: 24: 12.639 & 33.149 & -115.640 & 3.5 \\ 2010 / 01 / 28 & 20: 24: 47.639 & 33.157 & -115.680 & 0.2 \\ 2010 / 01 / 28 & 20: 25: 56.639 & 33.157 & -115.680 & 0.2 \\ 2010 / 01 / 28 & 20: 26: 51.639 & 33.157 & -115.680 & 0.2 \\ 2010 / 01 / 28 & 20: 30: 30.639 & 33.157 & -115.680 & 0.2 \\ 2010 / 01 / 28 & 20: 32: 18.639 & 33.157 & -115.680 & 0.2\end{array}$




$\begin{array}{lllll}2010 / 01 / 28 & 20: 52: 41.639 & 33.157 & -115.680 & 0.2 \\ 2010 / 01 / 28 & 20: 58: 19.639 & 33.184 & -115.601 & 3.5 \\ 2010 / 01 / 28 & 21: 07: 12.639 & 33.184 & -115.601 & 3.5 \\ 2010 / 01 / 28 & 21: 12: 36.639 & 33.142 & -115.628 & 10.3 \\ 2010 / 01 / 28 & 21: 30: 57.639 & 33.174 & -115.603 & 0.1 \\ 2010 / 01 / 28 & 22: 44: 43.639 & 33.168 & -115.609 & 0.7 \\ 2010 / 01 / 28 & 22: 46: 31.639 & 33.179 & -115.614 & 1.8 \\ 2010 / 01 / 28 & 22: 49: 11.639 & 33.168 & -115.609 & 0.7 \\ 2010 / 01 / 28 & 22: 53: 03.639 & 33.175 & -115.610 & 2.4 \\ 2010 / 01 / 28 & 22: 54: 31.639 & 33.168 & -115.609 & 0.7 \\ 2010 / 01 / 28 & 23: 00: 26.639 & 33.168 & -115.609 & 0.7 \\ 2010 / 01 / 28 & 23: 09: 41.639 & 33.179 & -115.614 & 1.8 \\ 2010 / 01 / 29 & 00: 58: 26.639 & 33.174 & -115.603 & 0.1 \\ 2010 / 01 / 29 & 01: 07: 28.639 & 33.174 & -115.603 & 0.1 \\ 2010 / 01 / 29 & 01: 44: 21.639 & 33.174 & -115.603 & 0.1 \\ 2010 / 01 / 29 & 01: 46: 06.639 & 33.180 & -115.600 & 0.1 \\ 2010 / 01 / 29 & 02: 58: 22.639 & 33.174 & -115.603 & 0.1 \\ 2010 / 01 / 29 & 04: 21: 12.639 & 33.175 & -115.610 & 2.4 \\ 2010 / 01 / 29 & 07: 37: 26.639 & 33.174 & -115.603 & 0.1 \\ 2010 / 01 / 29 & 08: 00: 30.639 & 33.174 & -115.603 & 0.1 \\ 2010 / 01 / 29 & 08: 15: 53.639 & 33.174 & -115.603 & 0.1 \\ 2010 / 01 / 29 & 08: 23: 55.639 & 33.174 & -115.603 & 0.1 \\ 2010 / 01 / 29 & 08: 42: 44.639 & 33.174 & -115.603 & 0.1 \\ 2010 / 01 / 29 & 08: 53: 31.639 & 33.174 & -115.603 & 0.1 \\ 2010 / 01 / 29 & 09: 02: 15.639 & 33.174 & -115.603 & 0.1 \\ 2010 / 01 / 29 & 12: 39: 46.639 & 33.177 & -115.635 & 5.3 \\ 2010 / 01 / 29 & 14: 31: 50.639 & 33.174 & -115.603 & 0.1 \\ 2010 / 01 / 29 & 14: 39: 21.639 & 33.176 & -115.608 & 0.4 \\ 2010 / 01 / 29 & 14: 40: 56.639 & 33.190 & -115.604 & 1.2 \\ 2010 / 01 / 29 & 14: 41: 46.639 & 33.197 & -115.596 & 3.0 \\ 2010 / 01 / 29 & 19: 05: 32.639 & 33.176 & -115.618 & 3.7 \\ 2010 / 01 / 30 & 03: 33: 05.639 & 33.172 & -115.611 & 3.7 \\ 2010 / 01 / 30 & 12: 14: 16.639 & 33.172 & -115.611 & 3.7 \\ 2010 / 01 / 30 & 15: 33: 26.639 & 33.174 & -115.603 & 0.1 \\ 2010 / 01 / 30 & 16: 20: 25.639 & 33.174 & -115.603 & 0.1 \\ 2010 / 01 / 30 & 18: 49: 35.639 & 33.212 & -115.610 & 4.1 \\ 2010 / 01 / 31 & 01: 03: 53.639 & 33.174 & -115.603 & 0.1 \\ 2010 / 01 / 31 & 01: 10: 45.639 & 33.174 & -115.603 & 0.1 \\ 2010 / 02 / 01 & 00: 13: 14.639 & 33.185 & -115.608 & 2.0 \\ 2010 / 02 / 01 & 00: 52: 45.639 & 33.212 & -115.590 & 7.2 \\ 2010 / 02 / 01 & 01: 13: 06.639 & 33.108 & -115.602 & 8.1 \\ 2010 / 02 / 01 & 03: 43: 09.639 & 33.193 & -115.632 & 3.5 \\ 2010 / 02 / 01 & 05: 56: 56.639 & 33.229 & -115.576 & 3.4 \\ 2010 / 02 / 01 & 16: 40: 36.639 & 33.174 & -115.603 & 0.1\end{array}$




$\begin{array}{lllll}2010 / 02 / 01 & 19: 25: 58.639 & 33.176 & -115.604 & 0.0 \\ 2010 / 02 / 01 & 19: 26: 41.639 & 33.176 & -115.604 & 0.0 \\ 2010 / 02 / 01 & 19: 50: 57.639 & 33.176 & -115.608 & 0.4 \\ 2010 / 02 / 01 & 19: 51: 40.639 & 33.176 & -115.608 & 0.4 \\ 2010 / 02 / 02 & 04: 05: 58.639 & 33.192 & -115.567 & 3.1 \\ 2010 / 02 / 02 & 04: 24: 30.639 & 33.192 & -115.567 & 3.1 \\ 2010 / 02 / 02 & 04: 30: 48.639 & 33.194 & -115.567 & 3.1 \\ 2010 / 02 / 02 & 04: 38: 33.639 & 33.188 & -115.573 & 0.2 \\ 2010 / 02 / 02 & 04: 43: 54.639 & 33.176 & -115.604 & 0.0 \\ 2010 / 02 / 02 & 04: 45: 53.639 & 33.226 & -115.588 & 4.6 \\ 2010 / 02 / 02 & 04: 51: 59.639 & 33.175 & -115.603 & 0.1 \\ 2010 / 02 / 02 & 07: 40: 47.639 & 33.159 & -115.635 & 7.3 \\ 2010 / 02 / 02 & 09: 07: 39.639 & 33.174 & -115.603 & 0.1 \\ 2010 / 02 / 02 & 10: 31: 11.639 & 33.174 & -115.603 & 0.1 \\ 2010 / 02 / 02 & 10: 38: 17.639 & 33.176 & -115.604 & 0.0 \\ 2010 / 02 / 02 & 10: 38: 51.639 & 33.176 & -115.604 & 0.0 \\ 2010 / 02 / 02 & 12: 07: 28.639 & 33.177 & -115.635 & 5.3 \\ 2010 / 02 / 02 & 12: 52: 54.639 & 33.179 & -115.594 & 1.5 \\ 2010 / 02 / 02 & 13: 04: 24.639 & 33.181 & -115.608 & 1.0 \\ 2010 / 02 / 02 & 13: 04: 43.639 & 33.176 & -115.608 & 0.4 \\ 2010 / 02 / 02 & 13: 05: 07.639 & 33.176 & -115.608 & 0.4 \\ 2010 / 02 / 02 & 13: 05: 34.639 & 33.181 & -115.611 & 0.4 \\ 2010 / 02 / 02 & 13: 33: 28.639 & 33.176 & -115.608 & 0.4 \\ 2010 / 02 / 02 & 13: 36: 49.639 & 33.177 & -115.606 & 0.1 \\ 2010 / 02 / 02 & 13: 46: 19.639 & 33.176 & -115.608 & 0.4 \\ 2010 / 02 / 02 & 13: 49: 02.639 & 33.176 & -115.608 & 0.4 \\ 2010 / 02 / 02 & 13: 49: 58.639 & 33.177 & -115.614 & 1.2 \\ 2010 / 02 / 02 & 13: 50: 38.639 & 33.176 & -115.604 & 0.5 \\ 2010 / 02 / 02 & 13: 51: 04.639 & 33.176 & -115.608 & 0.4 \\ 2010 / 02 / 02 & 13: 52: 05.639 & 33.170 & -115.600 & 1.1 \\ 2010 / 02 / 02 & 13: 52: 32.639 & 33.184 & -115.597 & 0.1 \\ 2010 / 02 / 02 & 13: 53: 06.639 & 33.179 & -115.594 & 1.5 \\ 2010 / 02 / 02 & 13: 54: 38.639 & 33.181 & -115.608 & 1.0 \\ 2010 / 02 / 02 & 13: 55: 44.639 & 33.176 & -115.609 & 2.1 \\ 2010 / 02 / 02 & 13: 56: 11.639 & 33.176 & -115.608 & 0.4 \\ 2010 / 02 / 02 & 13: 57: 58.639 & 33.176 & -115.604 & 0.5 \\ 2010 / 02 / 02 & 13: 58: 10.639 & 33.193 & -115.615 & 3.9 \\ 2010 / 02 / 02 & 14: 04: 11.639 & 33.181 & -115.659 & 6.0 \\ 2010 / 02 / 02 & 14: 04: 54.639 & 33.177 & -115.606 & 0.1 \\ 2010 / 02 / 02 & 14: 10: 31.639 & 33.181 & -115.659 & 6.0 \\ 2010 / 02 / 02 & 22: 04: 14.639 & 33.201 & -115.544 & 3.4 \\ 2010 / 02 / 02 & 22: 21: 45.639 & 33.236 & -115.573 & 5.0 \\ 203 / 03 & 02: 20: 38.639 & 33.175 & -115.603 & 0.1 \\ 22: 30: 18.639 & 33.168 & -115.609 & 0.7\end{array}$




$\begin{array}{lllll}2010 / 02 / 03 & 22: 56: 57.639 & 33.108 & -115.602 & 8.1 \\ 2010 / 02 / 04 & 10: 33: 25.639 & 33.183 & -115.599 & 0.1 \\ 2010 / 02 / 04 & 21: 53: 06.639 & 33.188 & -115.609 & 2.3 \\ 2010 / 02 / 05 & 07: 55: 09.639 & 33.181 & -115.608 & 1.0 \\ 2010 / 02 / 05 & 10: 13: 49.639 & 33.181 & -115.614 & 3.1 \\ 2010 / 02 / 05 & 10: 14: 28.639 & 33.177 & -115.607 & 4.2 \\ 2010 / 02 / 05 & 10: 43: 44.639 & 33.100 & -115.590 & 14.6 \\ 2010 / 02 / 05 & 10: 59: 15.639 & 33.172 & -115.622 & 4.0 \\ 2010 / 02 / 05 & 13: 04: 29.639 & 33.108 & -115.602 & 8.1 \\ 2010 / 02 / 05 & 13: 07: 01.639 & 33.184 & -115.605 & 0.1 \\ 2010 / 02 / 05 & 15: 08: 03.639 & 33.192 & -115.626 & 7.7 \\ 2010 / 02 / 05 & 15: 52: 02.639 & 33.108 & -115.602 & 8.1 \\ 2010 / 02 / 05 & 16: 27: 11.639 & 33.176 & -115.604 & 0.0 \\ 2010 / 02 / 05 & 19: 50: 00.639 & 33.201 & -115.544 & 3.4 \\ 2010 / 02 / 06 & 02: 31: 11.639 & 33.172 & -115.622 & 4.0 \\ 2010 / 02 / 06 & 02: 31: 32.639 & 33.176 & -115.602 & 3.2 \\ 2010 / 02 / 06 & 02: 31: 54.639 & 33.176 & -115.602 & 3.2 \\ 2010 / 02 / 06 & 02: 32: 50.639 & 33.193 & -115.583 & 5.9 \\ 2010 / 02 / 06 & 02: 36: 45.639 & 33.179 & -115.597 & 0.1 \\ 2010 / 02 / 06 & 02: 37: 30.639 & 33.187 & -115.601 & 1.7 \\ 2010 / 02 / 06 & 02: 38: 32.639 & 33.226 & -115.588 & 4.6 \\ 2010 / 02 / 06 & 02: 39: 41.639 & 33.186 & -115.593 & 0.1 \\ 2010 / 02 / 06 & 02: 40: 07.639 & 33.172 & -115.622 & 4.0 \\ 2010 / 02 / 06 & 14: 40: 27.639 & 33.181 & -115.602 & 0.4 \\ 2010 / 02 / 06 & 14: 41: 28.639 & 33.181 & -115.602 & 0.4 \\ 2010 / 02 / 06 & 15: 26: 42.639 & 33.175 & -115.610 & 2.4 \\ 2010 / 02 / 06 & 16: 04: 17.639 & 33.176 & -115.618 & 3.7 \\ 2010 / 02 / 06 & 16: 04: 57.639 & 33.176 & -115.618 & 3.7 \\ 2010 / 02 / 06 & 16: 28: 53.639 & 33.181 & -115.611 & 0.4 \\ 2010 / 02 / 06 & 16: 34: 51.639 & 33.226 & -115.588 & 4.6 \\ 2010 / 02 / 06 & 17: 26: 12.639 & 33.172 & -115.622 & 4.0 \\ 2010 / 02 / 06 & 18: 26: 01.639 & 33.193 & -115.615 & 3.9 \\ 2010 / 02 / 06 & 19: 08: 28.639 & 33.218 & -115.639 & 3.0 \\ 2010 / 02 / 06 & 20: 00: 24.639 & 33.176 & -115.618 & 3.7 \\ 2010 / 02 / 06 & 20: 23: 27.639 & 33.175 & -115.600 & 1.1 \\ 2010 / 02 / 06 & 20: 24: 05.639 & 33.175 & -115.600 & 1.1 \\ 2010 / 02 / 06 & 22: 19: 16.639 & 33.108 & -115.602 & 8.1 \\ 2010 / 02 / 06 & 22: 23: 07.639 & 33.158 & -115.642 & 0.1 \\ 2010 / 02 / 07 & 00: 25: 44.639 & 33.175 & -115.610 & 2.4 \\ 2010 / 02 / 07 & 00: 26: 46.639 & 33.175 & -115.600 & 1.1 \\ 2010 / 02 / 07 & 00: 27: 57.639 & 33.179 & -115.597 & 0.1 \\ 2010 / 02 / 07 & 00: 31: 32.639 & 33.179 & -115.597 & 0.1 \\ 2010 / 02 / 07 & 00: 47: 31.639 & 33.168 & -115.609 & 0.7 \\ 2010 / 02 / 07 & 00: 48: 52.639 & 33.175 & -115.600 & 1.1 \\ 2010 / 02 / 07 & 01: 58: 03.639 & 33.175 & -115.610 & 2.4 \\ 2010 / 02 / 07 & 02: 54: 25.639 & 33.175 & -115.610 & 2.4\end{array}$




$\begin{array}{lllll}2010 / 02 / 07 & 06: 46: 26.639 & 33.168 & -115.609 & 0.7 \\ 2010 / 02 / 07 & 09: 07: 17.639 & 33.172 & -115.622 & 4.0 \\ 2010 / 02 / 07 & 09: 42: 49.639 & 33.177 & -115.607 & 4.2 \\ 2010 / 02 / 07 & 09: 43: 41.639 & 33.176 & -115.602 & 3.2 \\ 2010 / 02 / 07 & 13: 53: 34.639 & 33.176 & -115.618 & 3.7 \\ 2010 / 02 / 07 & 14: 35: 55.639 & 33.176 & -115.618 & 3.7 \\ 2010 / 02 / 07 & 20: 07: 04.639 & 33.174 & -115.603 & 0.1 \\ 2010 / 02 / 07 & 21: 40: 56.639 & 33.176 & -115.608 & 0.4 \\ 2010 / 02 / 08 & 00: 59: 39.639 & 33.172 & -115.622 & 4.0 \\ 2010 / 02 / 08 & 01: 00: 19.639 & 33.175 & -115.601 & 0.1 \\ 2010 / 02 / 08 & 03: 43: 38.639 & 33.158 & -115.642 & 0.1 \\ 2010 / 02 / 08 & 06: 39: 01.639 & 33.118 & -115.610 & 9.1 \\ 2010 / 02 / 08 & 07: 51: 10.639 & 33.183 & -115.599 & 0.1 \\ 2010 / 02 / 08 & 08: 17: 45.639 & 33.183 & -115.599 & 0.1 \\ 2010 / 02 / 08 & 08: 22: 01.639 & 33.183 & -115.599 & 0.1 \\ 2010 / 02 / 08 & 08: 22: 50.639 & 33.183 & -115.599 & 0.1 \\ 2010 / 02 / 08 & 08: 37: 33.639 & 33.183 & -115.599 & 0.1 \\ 2010 / 02 / 08 & 09: 03: 17.639 & 33.183 & -115.599 & 0.1 \\ 2010 / 02 / 08 & 09: 20: 07.639 & 33.177 & -115.606 & 0.1 \\ 2010 / 02 / 08 & 10: 31: 54.639 & 33.181 & -115.603 & 0.1 \\ 2010 / 02 / 08 & 11: 22: 32.639 & 33.173 & -115.650 & 1.8 \\ 2010 / 02 / 08 & 12: 53: 45.639 & 33.158 & -115.638 & 0.4 \\ 2010 / 02 / 08 & 19: 10: 40.639 & 33.157 & -115.680 & 0.2 \\ 2010 / 02 / 08 & 20: 35: 06.639 & 33.192 & -115.579 & 4.3 \\ 2010 / 02 / 08 & 20: 54: 46.639 & 33.193 & -115.615 & 3.9 \\ 2010 / 02 / 08 & 21: 08: 36.639 & 33.188 & -115.599 & 0.8 \\ 2010 / 02 / 08 & 21: 11: 29.639 & 33.188 & -115.599 & 0.8 \\ 2010 / 02 / 08 & 21: 26: 28.639 & 33.185 & -115.569 & 3.7 \\ 2010 / 02 / 09 & 05: 04: 53.639 & 33.193 & -115.615 & 3.9 \\ 2010 / 02 / 09 & 13: 33: 52.639 & 33.176 & -115.609 & 3.8 \\ 2010 / 02 / 09 & 13: 40: 13.639 & 33.176 & -115.608 & 0.4 \\ 2010 / 02 / 09 & 15: 18: 57.639 & 33.193 & -115.583 & 5.9 \\ 2010 / 02 / 09 & 15: 51: 53.639 & 33.108 & -115.602 & 8.1 \\ 2010 / 02 / 09 & 22: 00: 55.639 & 33.180 & -115.600 & 0.1 \\ 2010 / 02 / 09 & 22: 58: 39.639 & 33.149 & -115.640 & 3.5 \\ 2010 / 02 / 09 & 23: 20: 24.639 & 33.149 & -115.640 & 3.5 \\ 2010 / 02 / 09 & 23: 23: 29.639 & 33.140 & -115.636 & 7.3 \\ 2010 / 02 / 10 & 00: 30: 26.639 & 33.184 & -115.606 & 2.9 \\ 2010 / 02 / 10 & 01: 06: 18.639 & 33.181 & -115.603 & 2.0 \\ 2010 / 02 / 10 & 01: 28: 28.639 & 33.184 & -115.606 & 2.9 \\ 2010 / 02 / 10 & 02: 31: 50.639 & 33.142 & -115.628 & 10.3 \\ 2010 / 02 / 10 & 07: 03: 34.639 & 33.108 & -115.602 & 8.1 \\ 2010 / 02 / 10 & 07: 47: 43.639 & 33.196 & -115.566 & 3.2 \\ 2010 / 02 / 10 & 12: 46: 48.639 & 33.176 & -115.604 & 0.5 \\ 2010 / 02 / 10 & 13: 08: 35.639 & 33.176 & -115.608 & 0.4 \\ 2010 / 02 / 10 & 13: 10: 31.639 & 33.176 & -115.604 & 0.5\end{array}$




$\begin{array}{lllll}2010 / 02 / 10 & 13: 20: 26.639 & 33.176 & -115.608 & 0.4 \\ 2010 / 02 / 10 & 13: 21: 36.639 & 33.229 & -115.645 & 5.7 \\ 2010 / 02 / 10 & 14: 09: 59.639 & 33.176 & -115.608 & 0.4 \\ 2010 / 02 / 10 & 16: 03: 21.639 & 33.180 & -115.600 & 0.1 \\ 2010 / 02 / 10 & 16: 18: 37.639 & 33.176 & -115.604 & 0.0 \\ 2010 / 02 / 10 & 16: 32: 44.639 & 33.100 & -115.590 & 14.6 \\ 2010 / 02 / 10 & 22: 36: 07.639 & 33.174 & -115.603 & 0.1 \\ 2010 / 02 / 10 & 22: 45: 15.639 & 33.174 & -115.603 & 0.1 \\ 2010 / 02 / 11 & 00: 54: 19.639 & 33.179 & -115.614 & 1.8 \\ 2010 / 02 / 11 & 03: 20: 47.639 & 33.168 & -115.609 & 0.7 \\ 2010 / 02 / 11 & 03: 21: 59.639 & 33.168 & -115.609 & 0.7 \\ 2010 / 02 / 11 & 03: 22: 22.639 & 33.168 & -115.609 & 0.7 \\ 2010 / 02 / 11 & 03: 29: 22.639 & 33.168 & -115.609 & 0.7 \\ 2010 / 02 / 11 & 09: 55: 12.639 & 33.181 & -115.659 & 6.0 \\ 2010 / 02 / 11 & 12: 13: 12.639 & 33.174 & -115.603 & 0.1 \\ 2010 / 02 / 11 & 12: 16: 29.639 & 33.174 & -115.603 & 0.1 \\ 2010 / 02 / 11 & 12: 16: 49.639 & 33.174 & -115.603 & 0.1 \\ 2010 / 02 / 11 & 12: 46: 02.639 & 33.218 & -115.639 & 3.0 \\ 2010 / 02 / 11 & 14: 23: 42.639 & 33.181 & -115.665 & 4.2 \\ 2010 / 02 / 11 & 14: 25: 15.639 & 33.229 & -115.576 & 3.4 \\ 2010 / 02 / 11 & 14: 49: 28.639 & 33.158 & -115.629 & 6.0 \\ 2010 / 02 / 11 & 14: 53: 12.639 & 33.199 & -115.571 & 3.3 \\ 2010 / 02 / 11 & 18: 00: 12.639 & 33.174 & -115.603 & 0.1 \\ 2010 / 02 / 11 & 23: 14: 35.639 & 33.179 & -115.614 & 1.8 \\ 2010 / 02 / 12 & 00: 38: 14.639 & 33.183 & -115.617 & 4.3 \\ 2010 / 02 / 12 & 09: 05: 15.639 & 33.188 & -115.609 & 2.3 \\ 2010 / 02 / 12 & 09: 14: 16.639 & 33.188 & -115.609 & 2.3 \\ 2010 / 02 / 12 & 09: 17: 47.639 & 33.188 & -115.609 & 2.3 \\ 2010 / 02 / 12 & 11: 50: 03.639 & 33.193 & -115.583 & 5.9 \\ 2010 / 02 / 12 & 15: 24: 15.639 & 33.188 & -115.573 & 1.2 \\ 2010 / 02 / 12 & 15: 58: 15.639 & 33.158 & -115.629 & 6.0 \\ 2010 / 02 / 12 & 18: 47: 27.639 & 33.181 & -115.665 & 4.2 \\ 2010 / 02 / 12 & 20: 49: 00.639 & 33.193 & -115.583 & 5.9 \\ 2010 / 02 / 13 & 02: 36: 51.639 & 33.175 & -115.603 & 0.1 \\ 2010 / 02 / 13 & 03: 45: 42.639 & 33.181 & -115.611 & 0.4 \\ 2010 / 02 / 13 & 03: 55: 44.639 & 33.181 & -115.611 & 0.4 \\ 2010 / 02 / 13 & 04: 12: 22.639 & 33.186 & -115.612 & 0.6 \\ 2010 / 02 / 13 & 04: 14: 53.639 & 33.181 & -115.611 & 0.4 \\ 2010 / 02 / 13 & 04: 17: 29.639 & 33.186 & -115.612 & 0.6 \\ 2010 / 02 / 13 & 04: 18: 32.639 & 33.251 & -115.564 & 0.1 \\ 2010 / 02 / 13 & 04: 33: 04.639 & 33.181 & -115.611 & 0.4 \\ 2010 / 02 / 13 & 04: 35: 59.639 & 33.181 & -115.611 & 0.4 \\ 2010 / 02 / 13 & 04: 36: 43.639 & 33.203 & -115.578 & 2.2 \\ 2010 / 02 / 13 & 04: 55: 47.639 & 33.186 & -115.612 & 0.6 \\ 2010 / 02 / 13 & 04: 56: 20.639 & 33.181 & -115.611 & 0.4 \\ 2010 / 02 / 13 & 04: 58: 14.639 & 33.181 & -115.611 & 0.4\end{array}$




$\begin{array}{lllll}2010 / 02 / 13 & 05: 00: 17.639 & 33.172 & -115.609 & 0.1 \\ 2010 / 02 / 13 & 05: 11: 32.639 & 33.181 & -115.611 & 0.4 \\ 2010 / 02 / 13 & 05: 13: 58.639 & 33.184 & -115.597 & 0.1 \\ 2010 / 02 / 13 & 05: 27: 18.639 & 33.181 & -115.608 & 1.0 \\ 2010 / 02 / 13 & 07: 29: 56.639 & 33.181 & -115.608 & 1.0 \\ 2010 / 02 / 13 & 11: 26: 02.639 & 33.108 & -115.602 & 8.1 \\ 2010 / 02 / 13 & 11: 26: 15.639 & 33.173 & -115.650 & 1.8 \\ 2010 / 02 / 13 & 16: 30: 45.639 & 33.199 & -115.617 & 0.0 \\ 2010 / 02 / 13 & 21: 26: 25.639 & 33.176 & -115.609 & 2.1 \\ 2010 / 02 / 13 & 21: 34: 33.639 & 33.165 & -115.623 & 7.0 \\ 2010 / 02 / 13 & 22: 29: 28.639 & 33.176 & -115.618 & 3.7 \\ 2010 / 02 / 13 & 23: 59: 50.639 & 33.176 & -115.602 & 3.2 \\ 2010 / 02 / 14 & 00: 00: 15.639 & 33.172 & -115.622 & 4.0 \\ 2010 / 02 / 14 & 00: 00: 18.639 & 33.108 & -115.602 & 8.1 \\ 2010 / 02 / 14 & 02: 20: 42.639 & 33.176 & -115.604 & 0.5 \\ 2010 / 02 / 14 & 04: 56: 55.639 & 33.180 & -115.603 & 0.1 \\ 2010 / 02 / 14 & 06: 58: 27.639 & 33.208 & -115.631 & 5.0 \\ 2010 / 02 / 14 & 07: 37: 38.639 & 33.184 & -115.597 & 0.1 \\ 2010 / 02 / 14 & 08: 59: 27.639 & 33.175 & -115.601 & 0.1 \\ 2010 / 02 / 14 & 12: 24: 57.639 & 33.231 & -115.657 & 2.0 \\ 2010 / 02 / 14 & 14: 41: 19.639 & 33.181 & -115.601 & 0.7 \\ 2010 / 02 / 14 & 19: 12: 33.639 & 33.208 & -115.582 & 2.5 \\ 2010 / 02 / 14 & 21: 06: 15.639 & 33.185 & -115.569 & 3.7 \\ 2010 / 02 / 14 & 21: 06: 52.639 & 33.186 & -115.593 & 0.1 \\ 2010 / 02 / 14 & 21: 23: 22.639 & 33.184 & -115.606 & 2.9 \\ 2010 / 02 / 14 & 21: 37: 26.639 & 33.174 & -115.603 & 0.1 \\ 2010 / 02 / 14 & 21: 37: 55.639 & 33.174 & -115.603 & 0.1 \\ 2010 / 02 / 14 & 22: 00: 42.639 & 33.248 & -115.633 & 2.9 \\ 2010 / 02 / 14 & 22: 30: 20.639 & 33.108 & -115.602 & 8.1 \\ 2010 / 02 / 14 & 23: 30: 26.639 & 33.176 & -115.604 & 0.5 \\ 2010 / 02 / 15 & 01: 47: 07.639 & 33.108 & -115.602 & 8.1 \\ 2010 / 02 / 15 & 05: 19: 58.639 & 33.184 & -115.609 & 2.2 \\ 2010 / 02 / 15 & 16: 04: 56.639 & 33.193 & -115.615 & 3.9 \\ 2010 / 02 / 15 & 16: 06: 54.639 & 33.188 & -115.609 & 2.3 \\ 2010 / 02 / 15 & 16: 32: 40.639 & 33.188 & -115.573 & 0.2 \\ 2010 / 02 / 15 & 18: 05: 09.639 & 33.188 & -115.609 & 2.3 \\ 2010 / 02 / 15 & 21: 37: 09.639 & 33.185 & -115.569 & 3.7 \\ 2010 / 02 / 16 & 01: 13: 29.639 & 33.176 & -115.604 & 0.0 \\ 2010 / 02 / 16 & 04: 34: 47.639 & 33.177 & -115.606 & 0.1 \\ 2010 / 02 / 16 & 05: 36: 56.639 & 33.177 & -115.606 & 0.1 \\ 2010 / 02 / 16 & 19: 56: 38.639 & 33.197 & -115.596 & 3.0 \\ 2010 / 02 / 16 & 23: 05: 23.639 & 33.108 & -115.602 & 8.1 \\ 2010 / 02 / 17 & 01: 22: 41.639 & 33.179 & -115.597 & 0.1 \\ 202 / 17 & 09: 53: 09.639 & 33.184 & -115.606 & 2.9 \\ 21: 55: 23.639 & 33.177 & -115.606 & 0.1\end{array}$




$\begin{array}{lllll}2010 / 02 / 17 & 13: 09: 10.639 & 33.181 & -115.612 & 1.1 \\ 2010 / 02 / 17 & 13: 10: 03.639 & 33.193 & -115.615 & 3.9 \\ 2010 / 02 / 17 & 13: 15: 29.639 & 33.181 & -115.612 & 1.1 \\ 2010 / 02 / 17 & 16: 03: 50.639 & 33.176 & -115.606 & 6.3 \\ 2010 / 02 / 17 & 16: 18: 04.639 & 33.183 & -115.617 & 4.3 \\ 2010 / 02 / 18 & 00: 40: 42.639 & 33.172 & -115.622 & 4.0 \\ 2010 / 02 / 18 & 02: 39: 39.639 & 33.191 & -115.684 & 3.3 \\ 2010 / 02 / 18 & 02: 40: 23.639 & 33.118 & -115.610 & 9.1 \\ 2010 / 02 / 18 & 03: 04: 03.639 & 33.176 & -115.609 & 2.1 \\ 2010 / 02 / 18 & 03: 30: 13.639 & 33.157 & -115.680 & 0.2 \\ 2010 / 02 / 18 & 05: 03: 17.639 & 33.183 & -115.599 & 0.1 \\ 2010 / 02 / 18 & 05: 35: 56.639 & 33.176 & -115.604 & 0.0 \\ 2010 / 02 / 18 & 07: 59: 13.639 & 33.188 & -115.573 & 0.2 \\ 2010 / 02 / 18 & 08: 09: 10.639 & 33.176 & -115.604 & 0.0 \\ 2010 / 02 / 18 & 09: 24: 32.639 & 33.183 & -115.599 & 0.1 \\ 2010 / 02 / 18 & 10: 35: 40.639 & 33.174 & -115.603 & 0.1 \\ 2010 / 02 / 18 & 10: 46: 57.639 & 33.176 & -115.604 & 0.0 \\ 2010 / 02 / 18 & 14: 39: 26.639 & 33.159 & -115.641 & 0.9 \\ 2010 / 02 / 18 & 15: 28: 28.639 & 33.231 & -115.657 & 2.0 \\ 2010 / 02 / 18 & 21: 49: 38.639 & 33.117 & -115.613 & 7.9 \\ 2010 / 02 / 18 & 21: 51: 31.639 & 33.117 & -115.613 & 7.9 \\ 2010 / 02 / 18 & 21: 51: 45.639 & 33.117 & -115.613 & 7.9 \\ 2010 / 02 / 18 & 21: 52: 59.639 & 33.158 & -115.638 & 0.4 \\ 2010 / 02 / 18 & 22: 01: 24.639 & 33.142 & -115.628 & 10.3 \\ 2010 / 02 / 18 & 23: 53: 38.639 & 33.170 & -115.600 & 1.1 \\ 2010 / 02 / 19 & 06: 06: 27.639 & 33.175 & -115.601 & 0.1 \\ 2010 / 02 / 19 & 13: 33: 30.639 & 33.199 & -115.617 & 0.0 \\ 2010 / 02 / 19 & 15: 08: 37.639 & 33.177 & -115.606 & 0.1 \\ 2010 / 02 / 19 & 15: 54: 50.639 & 33.149 & -115.640 & 3.5 \\ 2010 / 02 / 19 & 21: 31: 45.639 & 33.181 & -115.603 & 0.1 \\ 2010 / 02 / 19 & 22: 05: 03.639 & 33.181 & -115.603 & 2.0 \\ 2010 / 02 / 19 & 22: 20: 46.639 & 33.176 & -115.609 & 3.8 \\ 2010 / 02 / 20 & 00: 05: 00.639 & 33.149 & -115.640 & 3.5 \\ 2010 / 02 / 20 & 00: 12: 56.639 & 33.117 & -115.613 & 7.9 \\ 2010 / 02 / 20 & 00: 18: 50.639 & 33.165 & -115.623 & 7.0 \\ 2010 / 02 / 20 & 02: 11: 43.639 & 33.183 & -115.617 & 4.3 \\ 2010 / 02 / 20 & 10: 32: 14.639 & 33.175 & -115.606 & 4.2 \\ 2010 / 02 / 20 & 13: 00: 39.639 & 33.181 & -115.639 & 3.7 \\ 2010 / 02 / 20 & 13: 05: 24.639 & 33.181 & -115.639 & 3.7 \\ 2010 / 02 / 20 & 14: 30: 48.639 & 33.181 & -115.639 & 3.7 \\ 2010 / 02 / 20 & 17: 54: 05.639 & 33.196 & -115.587 & 5.3 \\ 2010 / 02 / 20 & 19: 26: 14.639 & 33.108 & -115.602 & 8.1 \\ 2010 / 02 / 20 & 20: 49: 55.639 & 33.173 & -115.650 & 1.8 \\ 2010 / 02 / 20 & 21: 22: 25.639 & 33.173 & -115.650 & 1.8 \\ 2010 / 02 / 20 & 21: 30: 26.639 & 33.173 & -115.650 & 1.8 \\ 2010 / 02 / 20 & 22: 40: 17.639 & 33.175 & -115.610 & 2.4\end{array}$




$\begin{array}{lllll}2010 / 02 / 21 & 02: 18: 53.639 & 33.176 & -115.604 & 0.5 \\ 2010 / 02 / 21 & 13: 19: 51.639 & 33.108 & -115.602 & 8.1 \\ 2010 / 02 / 21 & 14: 05: 31.639 & 33.179 & -115.594 & 1.5 \\ 2010 / 02 / 21 & 14: 05: 55.639 & 33.177 & -115.622 & 4.2 \\ 2010 / 02 / 21 & 14: 19: 48.639 & 33.180 & -115.609 & 0.0 \\ 2010 / 02 / 21 & 17: 04: 02.639 & 33.176 & -115.608 & 0.4 \\ 2010 / 02 / 21 & 17: 04: 36.639 & 33.182 & -115.602 & 2.5 \\ 2010 / 02 / 21 & 19: 47: 46.639 & 33.176 & -115.604 & 0.0 \\ 2010 / 02 / 21 & 20: 36: 47.639 & 33.174 & -115.603 & 0.1 \\ 2010 / 02 / 21 & 20: 40: 51.639 & 33.174 & -115.603 & 0.1 \\ 2010 / 02 / 22 & 01: 42: 50.639 & 33.164 & -115.632 & 2.6 \\ 2010 / 02 / 22 & 02: 13: 01.639 & 33.108 & -115.602 & 8.1 \\ 2010 / 02 / 22 & 09: 34: 03.639 & 33.183 & -115.617 & 4.3 \\ 2010 / 02 / 22 & 10: 01: 42.639 & 33.176 & -115.608 & 0.4 \\ 2010 / 02 / 22 & 10: 28: 01.639 & 33.176 & -115.608 & 0.4 \\ 2010 / 02 / 22 & 12: 21: 19.639 & 33.186 & -115.612 & 0.6 \\ 2010 / 02 / 22 & 12: 22: 25.639 & 33.186 & -115.612 & 0.6 \\ 2010 / 02 / 22 & 12: 23: 40.639 & 33.186 & -115.612 & 0.6 \\ 2010 / 02 / 22 & 12: 24: 44.639 & 33.190 & -115.612 & 1.0 \\ 2010 / 02 / 22 & 12: 26: 26.639 & 33.186 & -115.612 & 0.6 \\ 2010 / 02 / 22 & 12: 27: 02.639 & 33.179 & -115.594 & 1.5 \\ 2010 / 02 / 22 & 12: 27: 25.639 & 33.179 & -115.594 & 1.5 \\ 2010 / 02 / 22 & 12: 27: 40.639 & 33.179 & -115.594 & 1.5 \\ 2010 / 02 / 22 & 12: 29: 52.639 & 33.186 & -115.612 & 0.6 \\ 2010 / 02 / 22 & 12: 31: 46.639 & 33.186 & -115.612 & 0.6 \\ 2010 / 02 / 22 & 12: 32: 24.639 & 33.186 & -115.612 & 0.6 \\ 2010 / 02 / 22 & 12: 34: 12.639 & 33.173 & -115.610 & 1.3 \\ 2010 / 02 / 22 & 12: 37: 15.639 & 33.186 & -115.612 & 0.6 \\ 2010 / 02 / 22 & 12: 39: 14.639 & 33.190 & -115.612 & 1.0 \\ 2010 / 02 / 22 & 12: 44: 53.639 & 33.184 & -115.608 & 0.3 \\ 2010 / 02 / 22 & 12: 45: 17.639 & 33.190 & -115.612 & 1.0 \\ 2010 / 02 / 22 & 12: 45: 37.639 & 33.188 & -115.609 & 2.3 \\ 2010 / 02 / 22 & 12: 47: 41.639 & 33.188 & -115.609 & 2.3 \\ 2010 / 02 / 22 & 12: 50: 31.639 & 33.199 & -115.571 & 3.3 \\ 2010 / 02 / 22 & 13: 47: 26.639 & 33.139 & -115.602 & 7.0 \\ 2010 / 02 / 22 & 14: 25: 44.639 & 33.173 & -115.650 & 1.8 \\ 2010 / 02 / 22 & 14: 35: 22.639 & 33.170 & -115.600 & 1.1 \\ 2010 / 02 / 22 & 16: 16: 45.639 & 33.187 & -115.604 & 3.2 \\ 2010 / 02 / 22 & 17: 39: 45.639 & 33.218 & -115.639 & 3.0 \\ 2010 / 02 / 22 & 18: 31: 09.639 & 33.175 & -115.601 & 0.1 \\ 2010 / 02 / 22 & 21: 48: 29.639 & 33.117 & -115.613 & 7.9 \\ 22: 02: 17.639 & 33.181 & -115.610 & 0.9 \\ 22: 29: 46.639 & 33.184 & -115.608 & 0.3 \\ 23 & 00: 00: 20.639 & 33.157 & -115.669 & 0.6 \\ 202 & 02: 25: 39.639 & 33.229 & -115.576 & 3.4 \\ 20.639 & 33.251 & -115.564 & 0.1\end{array}$




$\begin{array}{lllll}2010 / 02 / 23 & 02: 34: 34.639 & 33.229 & -115.576 & 3.4 \\ 2010 / 02 / 23 & 02: 50: 33.639 & 33.181 & -115.608 & 1.0 \\ 2010 / 02 / 23 & 03: 22: 51.639 & 33.181 & -115.611 & 0.4 \\ 2010 / 02 / 23 & 03: 54: 43.639 & 33.181 & -115.608 & 1.0 \\ 2010 / 02 / 23 & 03: 55: 09.639 & 33.226 & -115.588 & 4.6 \\ 2010 / 02 / 23 & 03: 55: 28.639 & 33.184 & -115.597 & 0.1 \\ 2010 / 02 / 23 & 03: 57: 46.639 & 33.181 & -115.608 & 1.0 \\ 2010 / 02 / 23 & 09: 21: 53.639 & 33.190 & -115.612 & 1.0 \\ 2010 / 02 / 23 & 09: 48: 25.639 & 33.208 & -115.582 & 2.5 \\ 2010 / 02 / 23 & 14: 46: 27.639 & 33.236 & -115.573 & 5.0 \\ 2010 / 02 / 23 & 16: 21: 59.639 & 33.209 & -115.590 & 3.1 \\ 2010 / 02 / 23 & 16: 42: 20.639 & 33.176 & -115.609 & 2.1 \\ 2010 / 02 / 23 & 20: 21: 19.639 & 33.186 & -115.612 & 0.6 \\ 2010 / 02 / 23 & 21: 03: 08.639 & 33.160 & -115.633 & 4.6 \\ 2010 / 02 / 23 & 21: 03: 29.639 & 33.186 & -115.612 & 0.6 \\ 2010 / 02 / 23 & 21: 25: 43.639 & 33.161 & -115.644 & 1.0 \\ 2010 / 02 / 23 & 21: 27: 20.639 & 33.207 & -115.567 & 3.7 \\ 2010 / 02 / 23 & 22: 52: 08.639 & 33.214 & -115.620 & 5.3 \\ 2010 / 02 / 23 & 23: 40: 26.639 & 33.183 & -115.617 & 4.3 \\ 2010 / 02 / 23 & 23: 42: 58.639 & 33.193 & -115.615 & 3.9 \\ 2010 / 02 / 23 & 23: 50: 40.639 & 33.171 & -115.605 & 0.7 \\ 2010 / 02 / 24 & 00: 15: 02.639 & 33.184 & -115.604 & 0.1 \\ 2010 / 02 / 24 & 00: 44: 29.639 & 33.181 & -115.603 & 2.0 \\ 2010 / 02 / 24 & 02: 34: 01.639 & 33.161 & -115.644 & 1.0 \\ 2010 / 02 / 24 & 02: 34: 19.639 & 33.161 & -115.644 & 1.0 \\ 2010 / 02 / 24 & 02: 34: 54.639 & 33.161 & -115.644 & 1.0 \\ 2010 / 02 / 24 & 02: 35: 26.639 & 33.118 & -115.590 & 13.8 \\ 2010 / 02 / 24 & 02: 37: 20.639 & 33.161 & -115.644 & 1.0 \\ 2010 / 02 / 24 & 02: 58: 34.639 & 33.181 & -115.611 & 0.4 \\ 2010 / 02 / 24 & 03: 05: 52.639 & 33.183 & -115.617 & 4.3 \\ 2010 / 02 / 24 & 07: 20: 41.639 & 33.208 & -115.631 & 5.0 \\ 2010 / 02 / 24 & 11: 04: 38.639 & 33.188 & -115.573 & 0.2 \\ 2010 / 02 / 24 & 11: 56: 52.639 & 33.117 & -115.613 & 7.9 \\ 2010 / 02 / 24 & 12: 03: 06.639 & 33.183 & -115.617 & 4.3 \\ 2010 / 02 / 24 & 12: 32: 55.639 & 33.108 & -115.602 & 8.1 \\ 2010 / 02 / 24 & 16: 35: 33.639 & 33.184 & -115.606 & 2.9 \\ 2010 / 02 / 24 & 17: 12: 37.639 & 33.173 & -115.650 & 1.8 \\ 2010 / 02 / 24 & 22: 22: 34.639 & 33.142 & -115.628 & 10.3 \\ 2010 / 02 / 24 & 22: 24: 14.639 & 33.202 & -115.575 & 3.8 \\ 2010 / 02 / 24 & 22: 33: 28.639 & 33.202 & -115.575 & 3.8 \\ 2010 / 02 / 25 & 00: 00: 33.639 & 33.188 & -115.571 & 3.0 \\ 2010 / 02 / 25 & 00: 00: 33.639 & 33.188 & -115.571 & 3.0 \\ 2010 / 02 / 25 & 02: 41: 19.639 & 33.130 & -115.634 & 0.0 \\ 2010 / 02 / 25 & 07: 40: 19.639 & 33.218 & -115.639 & 3.0 \\ 2010 / 02 / 25 & 10: 39: 53.639 & 33.174 & -115.603 & 0.1 \\ 2010 / 02 / 25 & 13: 13: 36.639 & 33.176 & -115.602 & 3.2\end{array}$




$\begin{array}{lllll}2010 / 02 / 25 & 20: 53: 11.639 & 33.208 & -115.631 & 5.0 \\ 2010 / 02 / 25 & 22: 28: 34.639 & 33.179 & -115.594 & 1.5 \\ 2010 / 02 / 26 & 00: 30: 14.639 & 33.202 & -115.575 & 3.8 \\ 2010 / 02 / 26 & 07: 55: 10.639 & 33.231 & -115.657 & 2.0 \\ 2010 / 02 / 26 & 08: 35: 26.639 & 33.108 & -115.602 & 8.1 \\ 2010 / 02 / 26 & 09: 52: 01.639 & 33.174 & -115.603 & 0.1 \\ 2010 / 02 / 26 & 09: 55: 27.639 & 33.186 & -115.612 & 0.6 \\ 2010 / 02 / 26 & 13: 10: 03.639 & 33.193 & -115.583 & 5.9 \\ 2010 / 02 / 26 & 17: 12: 05.639 & 33.176 & -115.618 & 3.7 \\ 2010 / 02 / 26 & 17: 43: 19.639 & 33.118 & -115.590 & 13.8 \\ 2010 / 02 / 26 & 20: 08: 24.639 & 33.175 & -115.601 & 0.1 \\ 2010 / 02 / 26 & 22: 00: 48.639 & 33.175 & -115.601 & 0.1 \\ 2010 / 02 / 27 & 02: 18: 30.639 & 33.193 & -115.615 & 3.9 \\ 2010 / 02 / 27 & 04: 05: 23.639 & 33.198 & -115.593 & 2.3 \\ 2010 / 02 / 27 & 05: 09: 41.639 & 33.231 & -115.655 & 4.8 \\ 2010 / 02 / 27 & 07: 06: 39.639 & 33.175 & -115.606 & 4.2 \\ 2010 / 02 / 27 & 07: 29: 11.639 & 33.190 & -115.604 & 1.2 \\ 2010 / 02 / 27 & 08: 14: 42.639 & 33.193 & -115.632 & 3.5 \\ 2010 / 02 / 27 & 10: 14: 24.639 & 33.175 & -115.603 & 0.1 \\ 2010 / 02 / 27 & 10: 14: 35.639 & 33.234 & -115.604 & 4.8 \\ 2010 / 02 / 27 & 12: 08: 01.639 & 33.181 & -115.610 & 0.9 \\ 2010 / 02 / 27 & 12: 11: 58.639 & 33.177 & -115.606 & 0.1 \\ 2010 / 02 / 27 & 12: 13: 35.639 & 33.166 & -115.617 & 5.3 \\ 2010 / 02 / 27 & 15: 48: 17.639 & 33.200 & -115.590 & 3.5 \\ 2010 / 02 / 27 & 17: 56: 31.639 & 33.200 & -115.590 & 3.5 \\ 2010 / 02 / 27 & 18: 12: 23.639 & 33.166 & -115.617 & 5.3 \\ 2010 / 02 / 27 & 19: 42: 37.639 & 33.183 & -115.599 & 0.1 \\ 2010 / 02 / 27 & 21: 45: 01.639 & 33.176 & -115.604 & 0.5 \\ 2010 / 02 / 27 & 22: 30: 52.639 & 33.183 & -115.599 & 0.1 \\ 2010 / 02 / 27 & 22: 31: 33.639 & 33.177 & -115.606 & 0.1 \\ 2010 / 02 / 28 & 00: 28: 35.639 & 33.174 & -115.604 & 0.1 \\ 2010 / 02 / 28 & 01: 44: 49.639 & 33.184 & -115.601 & 3.5 \\ 2010 / 02 / 28 & 05: 51: 20.639 & 33.170 & -115.600 & 1.1 \\ 2010 / 02 / 28 & 05: 52: 10.639 & 33.181 & -115.608 & 1.0 \\ 2010 / 02 / 28 & 09: 34: 56.639 & 33.183 & -115.599 & 0.1 \\ 2010 / 02 / 28 & 11: 20: 16.639 & 33.166 & -115.617 & 5.3 \\ 2010 / 03 / 01 & 00: 07: 31.639 & 33.175 & -115.601 & 0.1 \\ 2010 / 03 / 01 & 00: 08: 42.639 & 33.179 & -115.594 & 1.5 \\ 2010 / 03 / 01 & 06: 25: 30.639 & 33.170 & -115.600 & 1.1 \\ 2010 / 03 / 01 & 07: 46: 22.639 & 33.173 & -115.650 & 1.8 \\ 2010 / 03 / 01 & 09: 57: 06.639 & 33.163 & -115.637 & 0.1 \\ 2010 / 03 / 01 & 10: 57: 50.639 & 33.181 & -115.665 & 4.2 \\ 2010 / 03 / 01 & 22: 10: 07.639 & 33.193 & -115.583 & 5.9 \\ 2010 / 03 / 02 & 23: 16: 01.639 & 33.203 & -115.578 & 2.2 \\ 2010 / 03 / 03 & 03: 04: 25.639 & 33.184 & -115.606 & 2.9 \\ 2010 / 03 / 03 & 07: 52: 32.639 & 33.199 & -115.617 & 0.0\end{array}$




\begin{tabular}{lllll}
$2010 / 03 / 03$ & $12: 31: 55.639$ & 33.117 & -115.613 & 7.9 \\
$2010 / 03 / 03$ & $13: 11: 18.639$ & 33.179 & -115.594 & 1.5 \\
$2010 / 03 / 03$ & $15: 16: 52.639$ & 33.208 & -115.582 & 2.5 \\
$2010 / 03 / 03$ & $15: 17: 41.639$ & 33.208 & -115.582 & 2.5 \\
$2010 / 03 / 03$ & $15: 56: 11.639$ & 33.130 & -115.634 & 0.0 \\
$2010 / 03 / 03$ & $16: 16: 55.639$ & 33.175 & -115.610 & 2.4 \\
$2010 / 03 / 03$ & $19: 28: 25.639$ & 33.196 & -115.587 & 5.3 \\
$2010 / 03 / 03$ & $19: 38: 14.639$ & 33.176 & -115.602 & 3.2 \\
$2010 / 03 / 03$ & $19: 38: 56.639$ & 33.181 & -115.665 & 4.2 \\
$2010 / 03 / 03$ & $19: 44: 31.639$ & 33.236 & -115.573 & 5.0 \\
$2010 / 03 / 03$ & $23: 44: 37.639$ & 33.236 & -115.573 & 5.0 \\
$2010 / 03 / 04$ & $01: 36: 52.639$ & 33.184 & -115.608 & 0.3 \\
$2010 / 03 / 04$ & $04: 04: 58.639$ & 33.182 & -115.603 & 0.1 \\
$2010 / 03 / 04$ & $04: 56: 51.639$ & 33.208 & -115.582 & 2.5 \\
$2010 / 03 / 04$ & $06: 32: 13.639$ & 33.117 & -115.613 & 7.9 \\
$2010 / 03 / 04$ & $08: 38: 59.639$ & 33.182 & -115.603 & 0.1 \\
$2010 / 03 / 04$ & $10: 01: 17.639$ & 33.184 & -115.606 & 2.9 \\
$2010 / 03 / 04$ & $10: 04: 19.639$ & 33.184 & -115.606 & 2.9 \\
$2010 / 03 / 04$ & $14: 54: 22.639$ & 33.176 & -115.609 & 3.8 \\
$2010 / 03 / 04$ & $16: 47: 58.639$ & 33.176 & -115.609 & 3.8 \\
$2010 / 03 / 04$ & $16: 48: 53.639$ & 33.176 & -115.609 & 3.8 \\
$2010 / 03 / 04$ & $16: 49: 37.639$ & 33.176 & -115.609 & 3.8 \\
$2010 / 03 / 04$ & $17: 43: 31.639$ & 33.176 & -115.604 & 0.5 \\
$2010 / 03 / 04$ & $17: 44: 28.639$ & 33.176 & -115.609 & 3.8 \\
$2010 / 03 / 04$ & $17: 45: 58.639$ & 33.176 & -115.609 & 3.8 \\
$2010 / 03 / 04$ & $17: 52: 32.639$ & 33.175 & -115.601 & 0.1 \\
$2010 / 03 / 04$ & $17: 53: 16.639$ & 33.176 & -115.604 & 0.5 \\
$2010 / 03 / 04$ & $17: 54: 29.639$ & 33.176 & -115.604 & 0.5 \\
$2010 / 03 / 04$ & $18: 24: 31.639$ & 33.193 & -115.583 & 5.9 \\
$2010 / 03 / 04$ & $18: 45: 08.639$ & 33.108 & -115.602 & 8.1 \\
$2010 / 03 / 04$ & $18: 45: 47.639$ & 33.149 & -115.640 & 3.5 \\
$2010 / 03 / 04$ & $19: 48: 21.639$ & 33.108 & -115.602 & 8.1 \\
$2010 / 03 / 04$ & $20: 22: 35.639$ & 33.108 & -115.602 & 8.1 \\
$2010 / 03 / 04$ & $21: 11: 30.639$ & 33.176 & -115.608 & 0.4 \\
$2010 / 03 / 04$ & $21: 12: 23.639$ & 33.176 & -115.608 & 0.4 \\
$2010 / 03 / 04$ & $21: 47: 46.639$ & 33.176 & -115.609 & 3.8 \\
$2010 / 03 / 04$ & $22: 16: 21.639$ & 33.176 & -115.608 & 0.4 \\
$2010 / 03 / 04$ & $22: 17: 30.639$ & 33.176 & -115.608 & 0.4 \\
$2010 / 03 / 05$ & $01: 40: 11.639$ & 33.108 & -115.602 & 8.1 \\
$2010 / 03 / 05$ & $03: 42: 51.639$ & 33.177 & -115.635 & 5.3 \\
$2010 / 03 / 05$ & $04: 27: 54.639$ & 33.179 & -115.594 & 1.5 \\
$2010 / 03 / 05$ & $06: 04: 54.639$ & 33.175 & -115.604 & 0.5 \\
$2010 / 03 / 05$ & $06: 05: 14.639$ & 33.179 & -115.594 & 0.1 \\
$2010 / 03 / 05$ & $06: 06: 49.639$ & 33.179 & -115.594 & 1.5 \\
\hline & $06: 11: 30.639$ & 33.182 & -115.602 & 2.5
\end{tabular}




$\begin{array}{lllll}2010 / 03 / 05 & 06: 11: 56.639 & 33.208 & -115.582 & 2.5 \\ 2010 / 03 / 05 & 06: 13: 34.639 & 33.182 & -115.602 & 2.5 \\ 2010 / 03 / 05 & 06: 35: 45.639 & 33.182 & -115.602 & 2.5 \\ 2010 / 03 / 05 & 06: 42: 47.639 & 33.175 & -115.606 & 4.2 \\ 2010 / 03 / 05 & 06: 58: 08.639 & 33.176 & -115.604 & 0.5 \\ 2010 / 03 / 05 & 07: 47: 45.639 & 33.175 & -115.606 & 4.2 \\ 2010 / 03 / 05 & 09: 03: 17.639 & 33.176 & -115.609 & 3.8 \\ 2010 / 03 / 05 & 09: 03: 46.639 & 33.176 & -115.609 & 3.8 \\ 2010 / 03 / 05 & 09: 04: 41.639 & 33.176 & -115.609 & 3.8 \\ 2010 / 03 / 05 & 09: 41: 58.639 & 33.184 & -115.605 & 0.1 \\ 2010 / 03 / 05 & 14: 54: 08.639 & 33.176 & -115.608 & 0.4 \\ 2010 / 03 / 05 & 16: 44: 10.639 & 33.190 & -115.608 & 2.2 \\ 2010 / 03 / 05 & 22: 31: 43.639 & 33.179 & -115.614 & 1.8 \\ 2010 / 03 / 05 & 23: 37: 36.639 & 33.192 & -115.572 & 1.6 \\ 2010 / 03 / 06 & 00: 30: 25.639 & 33.183 & -115.599 & 0.1 \\ 2010 / 03 / 06 & 02: 57: 19.639 & 33.190 & -115.574 & 3.1 \\ 2010 / 03 / 06 & 04: 52: 34.639 & 33.184 & -115.608 & 0.3 \\ 2010 / 03 / 06 & 04: 53: 02.639 & 33.184 & -115.608 & 0.3 \\ 2010 / 03 / 06 & 05: 02: 56.639 & 33.176 & -115.608 & 0.4 \\ 2010 / 03 / 06 & 05: 13: 15.639 & 33.187 & -115.601 & 1.7 \\ 2010 / 03 / 06 & 05: 17: 08.639 & 33.187 & -115.601 & 1.7 \\ 2010 / 03 / 06 & 06: 37: 16.639 & 33.177 & -115.635 & 5.3 \\ 2010 / 03 / 06 & 10: 26: 04.639 & 33.181 & -115.611 & 0.4 \\ 2010 / 03 / 06 & 12: 40: 33.639 & 33.176 & -115.609 & 3.8 \\ 2010 / 03 / 06 & 16: 04: 17.639 & 33.170 & -115.654 & 0.0 \\ 2010 / 03 / 06 & 16: 06: 15.639 & 33.192 & -115.572 & 1.6 \\ 2010 / 03 / 06 & 16: 18: 55.639 & 33.176 & -115.609 & 2.1 \\ 2010 / 03 / 06 & 19: 01: 32.639 & 33.184 & -115.597 & 0.1 \\ 2010 / 03 / 06 & 19: 26: 43.639 & 33.181 & -115.611 & 0.4 \\ 2010 / 03 / 06 & 19: 38: 29.639 & 33.184 & -115.597 & 0.1 \\ 2010 / 03 / 06 & 21: 44: 55.639 & 33.181 & -115.608 & 1.0 \\ 2010 / 03 / 06 & 21: 50: 20.639 & 33.181 & -115.611 & 0.4 \\ 2010 / 03 / 06 & 21: 52: 35.639 & 33.226 & -115.588 & 4.6 \\ 2010 / 03 / 06 & 22: 01: 33.639 & 33.181 & -115.611 & 0.4 \\ 2010 / 03 / 06 & 22: 02: 03.639 & 33.184 & -115.597 & 0.1 \\ 2010 / 03 / 06 & 22: 07: 39.639 & 33.184 & -115.597 & 0.1 \\ 2010 / 03 / 06 & 22: 16: 53.639 & 33.231 & -115.605 & 4.8 \\ 2010 / 03 / 06 & 23: 09: 14.639 & 33.186 & -115.612 & 0.6 \\ 2010 / 03 / 06 & 23: 51: 25.639 & 33.184 & -115.597 & 0.1 \\ 2010 / 03 / 06 & 23: 52: 40.639 & 33.184 & -115.597 & 0.1 \\ 2010 / 03 / 07 & 00: 27: 18.639 & 33.188 & -115.609 & 2.3 \\ 2010 / 03 / 07 & 00: 41: 45.639 & 33.181 & -115.608 & 1.0 \\ 2010 / 03 / 07 & 00: 46: 54.639 & 33.231 & -115.608 & 1.0 \\ 207 & 01: 01: 13.639 & 33.226 & -115.588 & 4.6\end{array}$




$\begin{array}{lllll}2010 / 03 / 07 & 01: 41: 17.639 & 33.177 & -115.606 & 0.1 \\ 2010 / 03 / 07 & 01: 41: 45.639 & 33.176 & -115.604 & 0.0 \\ 2010 / 03 / 07 & 02: 00: 01.639 & 33.175 & -115.601 & 0.1 \\ 2010 / 03 / 07 & 02: 12: 42.639 & 33.177 & -115.606 & 0.1 \\ 2010 / 03 / 07 & 02: 19: 34.639 & 33.231 & -115.605 & 4.8 \\ 2010 / 03 / 07 & 02: 21: 58.639 & 33.182 & -115.603 & 0.1 \\ 2010 / 03 / 07 & 02: 24: 18.639 & 33.177 & -115.606 & 0.1 \\ 2010 / 03 / 07 & 04: 09: 50.639 & 33.186 & -115.612 & 0.6 \\ 2010 / 03 / 07 & 07: 47: 24.639 & 33.229 & -115.576 & 3.4 \\ 2010 / 03 / 07 & 08: 12: 01.639 & 33.176 & -115.608 & 0.4 \\ 2010 / 03 / 07 & 09: 31: 41.639 & 33.183 & -115.606 & 0.1 \\ 2010 / 03 / 07 & 09: 33: 37.639 & 33.197 & -115.596 & 3.0 \\ 2010 / 03 / 07 & 09: 59: 13.639 & 33.176 & -115.609 & 3.8 \\ 2010 / 03 / 07 & 13: 47: 35.639 & 33.174 & -115.603 & 0.1 \\ 2010 / 03 / 07 & 19: 37: 34.639 & 33.130 & -115.634 & 0.0 \\ 2010 / 03 / 07 & 19: 39: 22.639 & 33.190 & -115.604 & 1.2 \\ 2010 / 03 / 07 & 21: 17: 59.639 & 33.130 & -115.634 & 0.0 \\ 2010 / 03 / 08 & 06: 18: 23.639 & 33.185 & -115.569 & 3.7 \\ 2010 / 03 / 08 & 06: 18: 23.639 & 33.201 & -115.544 & 3.4 \\ 2010 / 03 / 08 & 09: 16: 30.639 & 33.176 & -115.604 & 0.0 \\ 2010 / 03 / 08 & 11: 00: 58.639 & 33.184 & -115.609 & 2.2 \\ 2010 / 03 / 08 & 11: 23: 58.639 & 33.181 & -115.610 & 0.9 \\ 2010 / 03 / 08 & 21: 28: 40.639 & 33.177 & -115.635 & 5.3 \\ 2010 / 03 / 08 & 21: 30: 03.639 & 33.177 & -115.635 & 5.3 \\ 2010 / 03 / 08 & 23: 29: 42.639 & 33.183 & -115.607 & 0.0 \\ 2010 / 03 / 09 & 01: 18: 30.639 & 33.181 & -115.665 & 4.2 \\ 2010 / 03 / 09 & 04: 49: 39.639 & 33.174 & -115.603 & 0.1 \\ 2010 / 03 / 09 & 09: 54: 46.639 & 33.174 & -115.603 & 0.1 \\ 2010 / 03 / 09 & 14: 55: 18.639 & 33.182 & -115.602 & 2.5 \\ 2010 / 03 / 09 & 14: 55: 47.639 & 33.176 & -115.608 & 0.4 \\ 2010 / 03 / 09 & 16: 13: 39.639 & 33.170 & -115.600 & 1.1 \\ 2010 / 03 / 09 & 16: 14: 46.639 & 33.176 & -115.604 & 0.5 \\ 2010 / 03 / 09 & 16: 20: 35.639 & 33.176 & -115.604 & 0.5 \\ 2010 / 03 / 09 & 16: 21: 00.639 & 33.176 & -115.604 & 0.5 \\ 2010 / 03 / 09 & 16: 21: 34.639 & 33.181 & -115.665 & 4.2 \\ 2010 / 03 / 09 & 16: 21: 50.639 & 33.176 & -115.604 & 0.5 \\ 2010 / 03 / 09 & 16: 22: 01.639 & 33.176 & -115.609 & 2.1 \\ 2010 / 03 / 09 & 16: 24: 39.639 & 33.181 & -115.665 & 4.2 \\ 2010 / 03 / 09 & 16: 28: 04.639 & 33.173 & -115.650 & 1.8 \\ 2010 / 03 / 09 & 16: 34: 26.639 & 33.176 & -115.665 & 4.2 \\ 2010 / 03 / 09 & 18: 29: 03.639 & 33.176 & -115.604 & 0.5 \\ 2010 / 03 / 09 & 19: 20: 56.639 & 33.184 & -115.606 & 2.9 \\ 20: 49: 53.639 & 33.183 & -115.607 & 0.0 \\ 20 & 00: 36: 09.639 & 33.117 & -115.613 & 7.9\end{array}$




$\begin{array}{lllll}2010 / 03 / 10 & 00: 41: 18.639 & 33.173 & -115.650 & 1.8 \\ 2010 / 03 / 10 & 06: 12: 49.639 & 33.172 & -115.611 & 3.7 \\ 2010 / 03 / 10 & 06: 37: 54.639 & 33.181 & -115.665 & 4.2 \\ 2010 / 03 / 10 & 20: 51: 14.639 & 33.176 & -115.608 & 0.4 \\ 2010 / 03 / 10 & 20: 53: 42.639 & 33.197 & -115.596 & 3.0 \\ 2010 / 03 / 10 & 20: 54: 49.639 & 33.173 & -115.650 & 1.8 \\ 2010 / 03 / 10 & 21: 48: 04.639 & 33.176 & -115.609 & 3.8 \\ 2010 / 03 / 10 & 21: 48: 16.639 & 33.172 & -115.611 & 3.7 \\ 2010 / 03 / 10 & 21: 48: 50.639 & 33.176 & -115.609 & 3.8 \\ 2010 / 03 / 10 & 21: 49: 29.639 & 33.172 & -115.611 & 3.7 \\ 2010 / 03 / 10 & 22: 41: 45.639 & 33.176 & -115.609 & 3.8 \\ 2010 / 03 / 10 & 23: 35: 20.639 & 33.176 & -115.609 & 2.1 \\ 2010 / 03 / 11 & 05: 09: 59.639 & 33.173 & -115.650 & 1.8 \\ 2010 / 03 / 11 & 06: 16: 07.639 & 33.174 & -115.603 & 0.1 \\ 2010 / 03 / 11 & 06: 22: 08.639 & 33.176 & -115.608 & 0.4 \\ 2010 / 03 / 11 & 08: 30: 39.639 & 33.174 & -115.603 & 0.1 \\ 2010 / 03 / 11 & 09: 59: 09.639 & 33.214 & -115.620 & 5.3 \\ 2010 / 03 / 11 & 10: 56: 19.639 & 33.208 & -115.631 & 5.0 \\ 2010 / 03 / 11 & 12: 33: 50.639 & 33.177 & -115.606 & 0.1 \\ 2010 / 03 / 11 & 12: 34: 47.639 & 33.177 & -115.606 & 0.1 \\ 2010 / 03 / 11 & 12: 40: 32.639 & 33.177 & -115.606 & 0.1 \\ 2010 / 03 / 11 & 12: 42: 13.639 & 33.177 & -115.606 & 0.1 \\ 2010 / 03 / 11 & 12: 44: 01.639 & 33.177 & -115.606 & 0.1 \\ 2010 / 03 / 11 & 12: 45: 56.639 & 33.177 & -115.606 & 0.1 \\ 2010 / 03 / 11 & 12: 46: 17.639 & 33.177 & -115.606 & 0.1 \\ 2010 / 03 / 11 & 12: 47: 07.639 & 33.173 & -115.650 & 1.8 \\ 2010 / 03 / 11 & 14: 04: 21.639 & 33.177 & -115.606 & 0.1 \\ 2010 / 03 / 11 & 14: 10: 17.639 & 33.177 & -115.606 & 0.1 \\ 2010 / 03 / 11 & 17: 17: 39.639 & 33.173 & -115.650 & 1.8 \\ 2010 / 03 / 11 & 18: 07: 42.639 & 33.177 & -115.606 & 0.1 \\ 2010 / 03 / 11 & 18: 07: 55.639 & 33.177 & -115.606 & 0.1 \\ 2010 / 03 / 11 & 20: 01: 10.639 & 33.142 & -115.628 & 10.3 \\ 2010 / 03 / 11 & 20: 08: 08.639 & 33.158 & -115.642 & 0.1 \\ 2010 / 03 / 11 & 20: 35: 11.639 & 33.192 & -115.572 & 1.6 \\ 2010 / 03 / 11 & 20: 41: 25.639 & 33.170 & -115.600 & 1.1 \\ 2010 / 03 / 11 & 20: 44: 07.639 & 33.188 & -115.571 & 3.0 \\ 2010 / 03 / 11 & 20: 53: 00.639 & 33.186 & -115.612 & 0.6 \\ 2010 / 03 / 11 & 21: 22: 35.639 & 33.192 & -115.567 & 3.1 \\ 2010 / 03 / 11 & 21: 28: 06.639 & 33.200 & -115.590 & 3.5 \\ 2010 / 03 / 11 & 21: 28: 24.639 & 33.200 & -115.590 & 3.5 \\ 2010 / 03 / 12 & 00: 06: 52.639 & 33.175 & -115.601 & 0.1 \\ 2010 / 03 / 12 & 00: 48: 23.639 & 33.194 & -115.583 & 3.0 \\ 2010 / 03 / 12 & 05: 56: 31.639 & 33.181 & -115.629 & 4.0 \\ 2010 / 03 / 12 & 05: 57: 13.639 & 33.176 & -115.609 & 3.8 \\ 2010 / 03 / 12 & 07: 54: 38.639 & 33.173 & -115.650 & 1.8 \\ 2010 / 03 / 12 & 08: 31: 51.639 & 33.177 & -115.621 & 2.7\end{array}$




$\begin{array}{lllll}2010 / 03 / 12 & 11: 11: 36.639 & 33.181 & -115.659 & 6.0 \\ 2010 / 03 / 12 & 11: 35: 10.639 & 33.180 & -115.600 & 0.1 \\ 2010 / 03 / 12 & 11: 35: 37.639 & 33.200 & -115.590 & 3.5 \\ 2010 / 03 / 12 & 12: 34: 04.639 & 33.175 & -115.606 & 4.2 \\ 2010 / 03 / 12 & 13: 08: 09.639 & 33.176 & -115.608 & 0.4 \\ 2010 / 03 / 12 & 13: 09: 14.639 & 33.180 & -115.603 & 0.1 \\ 2010 / 03 / 12 & 15: 11: 48.639 & 33.188 & -115.599 & 0.8 \\ 2010 / 03 / 12 & 16: 15: 55.639 & 33.189 & -115.567 & 2.5 \\ 2010 / 03 / 12 & 18: 10: 28.639 & 33.176 & -115.609 & 2.1 \\ 2010 / 03 / 12 & 18: 10: 43.639 & 33.176 & -115.609 & 2.1 \\ 2010 / 03 / 12 & 18: 11: 34.639 & 33.143 & -115.638 & 4.8 \\ 2010 / 03 / 12 & 19: 16: 23.639 & 33.193 & -115.632 & 3.5 \\ 2010 / 03 / 12 & 19: 16: 54.639 & 33.193 & -115.632 & 3.5 \\ 2010 / 03 / 12 & 19: 19: 47.639 & 33.181 & -115.610 & 0.9 \\ 2010 / 03 / 12 & 19: 20: 34.639 & 33.140 & -115.636 & 7.3 \\ 2010 / 03 / 12 & 19: 21: 51.639 & 33.136 & -115.649 & 5.3 \\ 2010 / 03 / 12 & 19: 23: 59.639 & 33.181 & -115.602 & 0.4 \\ 2010 / 03 / 12 & 19: 46: 01.639 & 33.176 & -115.608 & 0.4 \\ 2010 / 03 / 12 & 21: 44: 14.639 & 33.193 & -115.615 & 3.9 \\ 2010 / 03 / 12 & 22: 45: 29.639 & 33.175 & -115.606 & 4.2 \\ 2010 / 03 / 13 & 03: 35: 31.639 & 33.176 & -115.608 & 0.4 \\ 2010 / 03 / 13 & 05: 01: 41.639 & 33.202 & -115.575 & 3.8 \\ 2010 / 03 / 13 & 06: 49: 54.639 & 33.176 & -115.604 & 0.5 \\ 2010 / 03 / 13 & 08: 13: 02.639 & 33.159 & -115.641 & 0.9 \\ 2010 / 03 / 13 & 08: 14: 15.639 & 33.158 & -115.642 & 0.1 \\ 2010 / 03 / 13 & 08: 15: 11.639 & 33.157 & -115.648 & 0.1 \\ 2010 / 03 / 13 & 08: 15: 41.639 & 33.157 & -115.648 & 0.1 \\ 2010 / 03 / 13 & 08: 16: 06.639 & 33.157 & -115.648 & 0.1 \\ 2010 / 03 / 13 & 08: 16: 47.639 & 33.159 & -115.641 & 0.9 \\ 2010 / 03 / 13 & 08: 18: 19.639 & 33.157 & -115.648 & 0.1 \\ 2010 / 03 / 13 & 08: 19: 16.639 & 33.159 & -115.641 & 0.9 \\ 2010 / 03 / 13 & 08: 24: 30.639 & 33.158 & -115.642 & 0.1 \\ 2010 / 03 / 13 & 08: 24: 48.639 & 33.135 & -115.644 & 6.9 \\ 2010 / 03 / 13 & 10: 32: 24.639 & 33.174 & -115.603 & 0.1 \\ 2010 / 03 / 13 & 11: 32: 18.639 & 33.177 & -115.606 & 0.1 \\ 2010 / 03 / 13 & 11: 33: 40.639 & 33.177 & -115.606 & 0.1 \\ 2010 / 03 / 13 & 11: 56: 18.639 & 33.177 & -115.606 & 0.1 \\ 2010 / 03 / 13 & 12: 41: 50.639 & 33.161 & -115.651 & 0.7 \\ 2010 / 03 / 13 & 12: 47: 54.639 & 33.186 & -115.612 & 0.6 \\ 2010 / 03 / 13 & 13: 03: 56.639 & 33.183 & -115.677 & 0.5 \\ 2010 / 03 / 13 & 13: 05: 29.639 & 33.183 & -115.677 & 0.5 \\ 2010 / 03 / 13 & 13: 06: 47.639 & 33.161 & -115.651 & 0.7 \\ 2: 08: 14.639 & 33.193 & -115.632 & 3.5 \\ 203 / 13 & 13: 10: 15.639 & 33.214 & -115.562 & 2.2\end{array}$




$\begin{array}{lllll}2010 / 03 / 13 & 13: 11: 56.639 & 33.231 & -115.657 & 2.0 \\ 2010 / 03 / 13 & 13: 12: 45.639 & 33.175 & -115.610 & 2.4 \\ 2010 / 03 / 13 & 13: 26: 32.639 & 33.183 & -115.677 & 0.5 \\ 2010 / 03 / 13 & 13: 30: 24.639 & 33.161 & -115.651 & 0.7 \\ 2010 / 03 / 13 & 13: 30: 46.639 & 33.193 & -115.632 & 3.5 \\ 2010 / 03 / 13 & 13: 37: 22.639 & 33.161 & -115.651 & 0.7 \\ 2010 / 03 / 13 & 13: 38: 59.639 & 33.161 & -115.651 & 0.7 \\ 2010 / 03 / 13 & 13: 39: 47.639 & 33.161 & -115.651 & 0.7 \\ 2010 / 03 / 13 & 13: 41: 35.639 & 33.161 & -115.651 & 0.7 \\ 2010 / 03 / 13 & 14: 06: 12.639 & 33.231 & -115.605 & 4.8 \\ 2010 / 03 / 13 & 14: 09: 49.639 & 33.183 & -115.677 & 0.5 \\ 2010 / 03 / 13 & 14: 15: 17.639 & 33.165 & -115.623 & 7.0 \\ 2010 / 03 / 13 & 14: 15: 50.639 & 33.189 & -115.619 & 2.2 \\ 2010 / 03 / 13 & 14: 16: 32.639 & 33.183 & -115.677 & 0.5 \\ 2010 / 03 / 13 & 14: 17: 30.639 & 33.161 & -115.651 & 0.7 \\ 2010 / 03 / 13 & 18: 25: 11.639 & 33.182 & -115.602 & 2.5 \\ 2010 / 03 / 13 & 18: 26: 31.639 & 33.176 & -115.608 & 0.4 \\ 2010 / 03 / 13 & 18: 55: 39.639 & 33.176 & -115.608 & 0.4 \\ 2010 / 03 / 13 & 21: 57: 14.639 & 33.181 & -115.665 & 4.2 \\ 2010 / 03 / 13 & 23: 09: 46.639 & 33.226 & -115.588 & 4.6 \\ 2010 / 03 / 14 & 00: 36: 18.639 & 33.182 & -115.603 & 0.1 \\ 2010 / 03 / 14 & 00: 40: 57.639 & 33.177 & -115.606 & 0.1 \\ 2010 / 03 / 14 & 01: 38: 06.639 & 33.181 & -115.603 & 1.4 \\ 2010 / 03 / 14 & 02: 45: 58.639 & 33.118 & -115.610 & 9.1 \\ 2010 / 03 / 14 & 05: 27: 57.639 & 33.174 & -115.603 & 0.1 \\ 2010 / 03 / 14 & 07: 17: 23.639 & 33.157 & -115.668 & 0.1 \\ 2010 / 03 / 14 & 16: 52: 34.639 & 33.108 & -115.602 & 8.1 \\ 2010 / 03 / 14 & 16: 54: 25.639 & 33.108 & -115.602 & 8.1 \\ 2010 / 03 / 14 & 19: 13: 30.639 & 33.193 & -115.615 & 3.9 \\ 2010 / 03 / 14 & 20: 44: 38.639 & 33.175 & -115.601 & 0.1 \\ 2010 / 03 / 14 & 20: 53: 02.639 & 33.175 & -115.601 & 0.1 \\ 2010 / 03 / 15 & 00: 36: 20.639 & 33.176 & -115.609 & 2.1 \\ 2010 / 03 / 15 & 01: 36: 43.639 & 33.185 & -115.569 & 3.7 \\ 2010 / 03 / 15 & 04: 48: 12.639 & 33.170 & -115.600 & 1.1 \\ 2010 / 03 / 15 & 13: 08: 03.639 & 33.183 & -115.599 & 0.1 \\ 2010 / 03 / 15 & 13: 52: 05.639 & 33.175 & -115.601 & 0.1 \\ 2010 / 03 / 15 & 15: 01: 33.639 & 33.100 & -115.590 & 14.6 \\ 2010 / 03 / 15 & 15: 04: 11.639 & 33.108 & -115.602 & 8.1 \\ 2010 / 03 / 15 & 15: 29: 50.639 & 33.130 & -115.634 & 0.0 \\ 2010 / 03 / 15 & 15: 30: 03.639 & 33.108 & -115.602 & 8.1 \\ 2010 / 03 / 15 & 18: 23: 10.639 & 33.209 & -115.590 & 3.1 \\ 2010 / 03 / 16 & 00: 14: 57.639 & 33.181 & -115.608 & 1.0 \\ 2010 / 03 / 16 & 13: 36: 21.639 & 33.214 & -115.620 & 5.3 \\ 2010 / 03 / 16 & 19: 38: 12.639 & 33.108 & -115.602 & 8.1 \\ 2010 / 03 / 17 & 01: 33: 00.639 & 33.183 & -115.617 & 4.3\end{array}$




$\begin{array}{lllll}2010 / 03 / 17 & 05: 49: 28.639 & 33.183 & -115.599 & 0.1 \\ 2010 / 03 / 17 & 05: 50: 26.639 & 33.183 & -115.599 & 0.1 \\ 2010 / 03 / 17 & 05: 52: 01.639 & 33.183 & -115.599 & 0.1 \\ 2010 / 03 / 17 & 05: 53: 06.639 & 33.183 & -115.599 & 0.1 \\ 2010 / 03 / 17 & 06: 11: 51.639 & 33.183 & -115.599 & 0.1 \\ 2010 / 03 / 17 & 06: 14: 02.639 & 33.183 & -115.599 & 0.1 \\ 2010 / 03 / 17 & 06: 22: 39.639 & 33.183 & -115.599 & 0.1 \\ 2010 / 03 / 17 & 06: 30: 44.639 & 33.183 & -115.599 & 0.1 \\ 2010 / 03 / 17 & 06: 34: 20.639 & 33.229 & -115.576 & 3.4 \\ 2010 / 03 / 17 & 08: 30: 32.639 & 33.100 & -115.590 & 14.6 \\ 2010 / 03 / 17 & 10: 54: 07.639 & 33.226 & -115.588 & 4.6 \\ 2010 / 03 / 17 & 11: 03: 50.639 & 33.181 & -115.665 & 4.2 \\ 2010 / 03 / 17 & 14: 46: 00.639 & 33.179 & -115.594 & 1.5 \\ 2010 / 03 / 17 & 21: 47: 56.639 & 33.184 & -115.597 & 0.1 \\ 2010 / 03 / 17 & 23: 54: 23.639 & 33.180 & -115.603 & 0.1 \\ 2010 / 03 / 17 & 23: 54: 35.639 & 33.180 & -115.603 & 0.1 \\ 2010 / 03 / 17 & 23: 56: 32.639 & 33.198 & -115.593 & 2.3 \\ 2010 / 03 / 18 & 00: 22: 20.639 & 33.176 & -115.604 & 0.5 \\ 2010 / 03 / 18 & 02: 23: 38.639 & 33.108 & -115.602 & 8.1 \\ 2010 / 03 / 18 & 02: 48: 06.639 & 33.181 & -115.612 & 1.1 \\ 2010 / 03 / 18 & 08: 47: 59.639 & 33.176 & -115.604 & 0.5 \\ 2010 / 03 / 18 & 08: 49: 14.639 & 33.176 & -115.604 & 0.5 \\ 2010 / 03 / 18 & 08: 51: 21.639 & 33.183 & -115.607 & 0.0 \\ 2010 / 03 / 18 & 10: 03: 19.639 & 33.175 & -115.603 & 0.1 \\ 2010 / 03 / 18 & 16: 58: 30.639 & 33.173 & -115.650 & 1.8 \\ 2010 / 03 / 18 & 16: 59: 57.639 & 33.173 & -115.650 & 1.8 \\ 2010 / 03 / 18 & 18: 24: 06.639 & 33.208 & -115.582 & 2.5 \\ 2010 / 03 / 18 & 18: 48: 57.639 & 33.190 & -115.574 & 3.1 \\ 2010 / 03 / 19 & 02: 14: 37.639 & 33.108 & -115.602 & 8.1 \\ 2010 / 03 / 19 & 03: 21: 00.639 & 33.181 & -115.659 & 6.0 \\ 2010 / 03 / 19 & 11: 05: 21.639 & 33.208 & -115.631 & 5.0 \\ 2010 / 03 / 19 & 23: 01: 08.639 & 33.108 & -115.602 & 8.1 \\ 2010 / 03 / 19 & 23: 03: 18.639 & 33.108 & -115.602 & 8.1 \\ 2010 / 03 / 19 & 23: 18: 27.639 & 33.186 & -115.604 & 2.0 \\ 2010 / 03 / 20 & 00: 48: 11.639 & 33.147 & -115.623 & 7.9 \\ 2010 / 03 / 20 & 01: 33: 17.639 & 33.108 & -115.602 & 8.1 \\ 2010 / 03 / 20 & 02: 49: 46.639 & 33.184 & -115.606 & 2.9 \\ 2010 / 03 / 20 & 03: 59: 08.639 & 33.108 & -115.602 & 8.1 \\ 2010 / 03 / 20 & 04: 53: 29.639 & 33.140 & -115.636 & 7.3 \\ 2010 / 03 / 20 & 10: 17: 50.639 & 33.100 & -115.590 & 14.6 \\ 2010 / 03 / 20 & 18: 29: 45.639 & 33.229 & -115.576 & 3.4 \\ 2010 / 03 / 20 & 18: 35: 25.639 & 33.183 & -115.606 & 0.1 \\ 2010 / 03 / 20 & 18: 40: 22.639 & 33.173 & -115.650 & 1.8 \\ 2010 / 03 / 20 & 18: 47: 47.639 & 33.187 & -115.608 & 0.2 \\ 2010 / 03 / 20 & 18: 48: 15.639 & 33.187 & -115.608 & 0.2 \\ 2010 / 03 / 20 & 18: 49: 55.639 & 33.229 & -115.576 & 3.4\end{array}$




$\begin{array}{lllll}2010 / 03 / 20 & 19: 01: 30.639 & 33.196 & -115.587 & 5.3 \\ 2010 / 03 / 20 & 19: 55: 34.639 & 33.183 & -115.606 & 0.1 \\ 2010 / 03 / 20 & 23: 05: 57.639 & 33.229 & -115.576 & 3.4 \\ 2010 / 03 / 21 & 00: 11: 30.639 & 33.117 & -115.613 & 7.9 \\ 2010 / 03 / 21 & 01: 51: 25.639 & 33.184 & -115.597 & 0.1 \\ 2010 / 03 / 21 & 02: 36: 11.639 & 33.175 & -115.606 & 4.2 \\ 2010 / 03 / 21 & 03: 25: 49.639 & 33.175 & -115.606 & 4.2 \\ 2010 / 03 / 21 & 03: 40: 41.639 & 33.183 & -115.599 & 0.1 \\ 2010 / 03 / 21 & 03: 46: 20.639 & 33.183 & -115.599 & 0.1 \\ 2010 / 03 / 22 & 02: 44: 06.639 & 33.180 & -115.600 & 0.1 \\ 2010 / 03 / 22 & 04: 59: 01.639 & 33.100 & -115.590 & 14.6 \\ 2010 / 03 / 22 & 09: 00: 45.639 & 33.180 & -115.600 & 0.1 \\ 2010 / 03 / 22 & 09: 01: 58.639 & 33.180 & -115.600 & 0.1 \\ 2010 / 03 / 22 & 20: 03: 36.639 & 33.190 & -115.574 & 3.1 \\ 2010 / 03 / 23 & 12: 18: 30.639 & 33.186 & -115.614 & 2.4 \\ 2010 / 03 / 23 & 18: 04: 41.639 & 33.190 & -115.574 & 3.1 \\ 2010 / 03 / 23 & 20: 43: 38.639 & 33.130 & -115.634 & 0.0 \\ 2010 / 03 / 24 & 05: 42: 00.639 & 33.174 & -115.603 & 0.1 \\ 2010 / 03 / 24 & 14: 43: 29.639 & 33.199 & -115.570 & 0.2 \\ 2010 / 03 / 25 & 01: 18: 48.639 & 33.174 & -115.603 & 0.1 \\ 2010 / 03 / 25 & 10: 19: 14.639 & 33.208 & -115.631 & 5.0 \\ 2010 / 03 / 25 & 11: 54: 36.639 & 33.170 & -115.600 & 1.1 \\ 2010 / 03 / 25 & 14: 54: 36.639 & 33.193 & -115.583 & 5.9 \\ 2010 / 03 / 25 & 21: 23: 02.639 & 33.183 & -115.617 & 4.3 \\ 2010 / 03 / 25 & 21: 24: 26.639 & 33.170 & -115.600 & 1.1 \\ 2010 / 03 / 26 & 04: 41: 55.639 & 33.161 & -115.644 & 1.0 \\ 2010 / 03 / 26 & 04: 43: 48.639 & 33.186 & -115.612 & 0.6 \\ 2010 / 03 / 26 & 05: 07: 53.639 & 33.135 & -115.644 & 6.9 \\ 2010 / 03 / 26 & 05: 12: 54.639 & 33.181 & -115.665 & 4.2 \\ 2010 / 03 / 26 & 08: 07: 53.639 & 33.108 & -115.602 & 8.1 \\ 2010 / 03 / 26 & 09: 55: 37.639 & 33.186 & -115.612 & 0.6 \\ 2010 / 03 / 26 & 14: 58: 31.639 & 33.229 & -115.645 & 5.7 \\ 2010 / 03 / 26 & 14: 58: 46.639 & 33.229 & -115.645 & 5.7 \\ 2010 / 03 / 26 & 14: 59: 33.639 & 33.229 & -115.576 & 3.4 \\ 2010 / 03 / 26 & 15: 00: 02.639 & 33.229 & -115.645 & 5.7 \\ 2010 / 03 / 26 & 23: 41: 10.639 & 33.173 & -115.650 & 1.8 \\ 2010 / 03 / 27 & 04: 08: 51.639 & 33.186 & -115.612 & 0.6 \\ 2010 / 03 / 27 & 13: 16: 13.639 & 33.183 & -115.617 & 4.3 \\ 2010 / 03 / 27 & 13: 23: 23.639 & 33.149 & -115.640 & 3.5 \\ 2010 / 03 / 27 & 13: 29: 05.639 & 33.201 & -115.544 & 3.4 \\ 2010 / 03 / 27 & 13: 32: 20.639 & 33.149 & -115.640 & 3.5 \\ 2010 / 03 / 27 & 13: 32: 51.639 & 33.183 & -115.599 & 0.1 \\ 2010 / 03 / 27 & 13: 35: 06.639 & 33.183 & -115.617 & 4.3 \\ 2010 / 03 / 27 & 13: 48: 42.639 & 33.208 & -115.631 & 5.0 \\ 2010 / 03 / 27 & 14: 52: 03.639 & 33.236 & -115.573 & 5.0 \\ 2010 / 03 / 27 & 17: 31: 56.639 & 33.212 & -115.633 & 7.6\end{array}$




$\begin{array}{lllll}2010 / 03 / 27 & 18: 22: 24.639 & 33.196 & -115.618 & 7.3 \\ 2010 / 03 / 27 & 23: 05: 58.639 & 33.176 & -115.608 & 0.4 \\ 2010 / 03 / 27 & 23: 22: 46.639 & 33.135 & -115.644 & 6.9 \\ 2010 / 03 / 27 & 23: 30: 27.639 & 33.135 & -115.644 & 6.9 \\ 2010 / 03 / 27 & 23: 31: 26.639 & 33.135 & -115.644 & 6.9 \\ 2010 / 03 / 27 & 23: 45: 52.639 & 33.142 & -115.628 & 10.3 \\ 2010 / 03 / 27 & 23: 49: 24.639 & 33.135 & -115.644 & 6.9 \\ 2010 / 03 / 28 & 00: 10: 32.639 & 33.108 & -115.602 & 8.1 \\ 2010 / 03 / 28 & 02: 25: 30.639 & 33.177 & -115.606 & 0.1 \\ 2010 / 03 / 28 & 08: 27: 36.639 & 33.177 & -115.635 & 5.3 \\ 2010 / 03 / 28 & 09: 34: 30.639 & 33.202 & -115.575 & 3.8 \\ 2010 / 03 / 28 & 09: 42: 49.639 & 33.193 & -115.602 & 0.0 \\ 2010 / 03 / 28 & 09: 49: 20.639 & 33.193 & -115.602 & 0.0 \\ 2010 / 03 / 28 & 09: 52: 26.639 & 33.193 & -115.602 & 0.0 \\ 2010 / 03 / 28 & 13: 27: 26.639 & 33.176 & -115.618 & 3.7 \\ 2010 / 03 / 28 & 23: 46: 24.639 & 33.196 & -115.587 & 4.5 \\ 2010 / 03 / 29 & 02: 29: 38.639 & 33.176 & -115.604 & 0.0 \\ 2010 / 03 / 29 & 05: 19: 44.639 & 33.177 & -115.606 & 0.1 \\ 2010 / 03 / 29 & 05: 20: 15.639 & 33.184 & -115.604 & 0.1 \\ 2010 / 03 / 29 & 05: 21: 50.639 & 33.177 & -115.606 & 0.1 \\ 2010 / 03 / 29 & 05: 39: 33.639 & 33.208 & -115.631 & 5.0 \\ 2010 / 03 / 29 & 09: 11: 35.639 & 33.184 & -115.606 & 2.9 \\ 2010 / 03 / 29 & 09: 14: 33.639 & 33.184 & -115.606 & 2.9 \\ 2010 / 03 / 29 & 10: 48: 25.639 & 33.183 & -115.677 & 0.5 \\ 2010 / 03 / 29 & 11: 29: 18.639 & 33.184 & -115.606 & 2.9 \\ 2010 / 03 / 29 & 15: 53: 33.639 & 33.174 & -115.603 & 0.1 \\ 2010 / 03 / 29 & 15: 57: 00.639 & 33.174 & -115.603 & 0.1 \\ 2010 / 03 / 29 & 15: 58: 36.639 & 33.174 & -115.603 & 0.1 \\ 2010 / 03 / 29 & 15: 59: 24.639 & 33.174 & -115.603 & 0.1 \\ 2010 / 03 / 29 & 15: 59: 47.639 & 33.174 & -115.603 & 0.1 \\ 2010 / 03 / 29 & 16: 04: 54.639 & 33.184 & -115.606 & 2.9 \\ 2010 / 03 / 29 & 16: 09: 11.639 & 33.174 & -115.603 & 0.1 \\ 2010 / 03 / 29 & 16: 24: 37.639 & 33.176 & -115.604 & 0.0 \\ 2010 / 03 / 29 & 16: 37: 25.639 & 33.174 & -115.603 & 0.1 \\ 2010 / 03 / 29 & 17: 10: 09.639 & 33.188 & -115.609 & 2.3 \\ 2010 / 03 / 29 & 17: 39: 03.639 & 33.184 & -115.604 & 0.1 \\ 2010 / 03 / 29 & 18: 46: 49.639 & 33.176 & -115.604 & 0.0 \\ 2010 / 03 / 29 & 19: 06: 22.639 & 33.174 & -115.603 & 0.1 \\ 2010 / 03 / 29 & 19: 08: 42.639 & 33.173 & -115.650 & 1.8 \\ 2010 / 03 / 29 & 19: 38: 20.639 & 33.184 & -115.608 & 0.3 \\ 2010 / 03 / 29 & 20: 03: 28.639 & 33.184 & -115.606 & 2.9 \\ 2010 / 03 / 29 & 21: 02: 06.639 & 33.176 & -115.606 & 6.3 \\ 2010 / 03 / 29 & 23: 38: 48.639 & 33.176 & -115.604 & 0.0 \\ 2010 / 03 / 29 & 23: 50: 00.639 & 33.176 & -115.604 & 0.0 \\ 2010 / 03 / 30 & 08: 05: 32.639 & 33.207 & -115.567 & 3.7\end{array}$




$\begin{array}{lllll}2010 / 03 / 30 & 10: 10: 47.639 & 33.176 & -115.604 & 0.0 \\ 2010 / 03 / 30 & 11: 23: 01.639 & 33.181 & -115.665 & 4.2 \\ 2010 / 03 / 30 & 11: 58: 28.639 & 33.183 & -115.599 & 0.1 \\ 2010 / 03 / 30 & 18: 03: 27.639 & 33.208 & -115.582 & 2.5 \\ 2010 / 03 / 30 & 18: 35: 35.639 & 33.234 & -115.604 & 4.8 \\ 2010 / 03 / 30 & 18: 36: 25.639 & 33.234 & -115.604 & 4.8 \\ 2010 / 03 / 30 & 18: 36: 37.639 & 33.184 & -115.604 & 0.1 \\ 2010 / 03 / 30 & 18: 37: 49.639 & 33.184 & -115.604 & 0.1 \\ 2010 / 03 / 30 & 18: 43: 09.639 & 33.185 & -115.569 & 3.7 \\ 2010 / 03 / 30 & 23: 10: 39.639 & 33.187 & -115.601 & 1.7 \\ 2010 / 03 / 31 & 02: 36: 36.639 & 33.142 & -115.628 & 10.3 \\ 2010 / 03 / 31 & 10: 36: 24.639 & 33.184 & -115.606 & 2.9 \\ 2010 / 03 / 31 & 10: 37: 04.639 & 33.174 & -115.603 & 0.1 \\ 2010 / 03 / 31 & 14: 16: 47.639 & 33.175 & -115.603 & 0.1 \\ 2010 / 03 / 31 & 17: 04: 12.639 & 33.183 & -115.599 & 0.1 \\ 2010 / 03 / 31 & 17: 05: 05.639 & 33.166 & -115.617 & 5.3 \\ 2010 / 03 / 31 & 17: 05: 56.639 & 33.177 & -115.606 & 0.1 \\ 2010 / 03 / 31 & 17: 06: 55.639 & 33.176 & -115.618 & 3.7 \\ 2010 / 03 / 31 & 17: 07: 29.639 & 33.183 & -115.599 & 0.1 \\ 2010 / 03 / 31 & 17: 09: 18.639 & 33.173 & -115.609 & 0.1 \\ 2010 / 03 / 31 & 17: 09: 30.639 & 33.198 & -115.586 & 3.7 \\ 2010 / 03 / 31 & 17: 11: 12.639 & 33.173 & -115.609 & 0.1 \\ 2010 / 03 / 31 & 17: 12: 10.639 & 33.182 & -115.606 & 1.7 \\ 2010 / 03 / 31 & 17: 15: 04.639 & 33.174 & -115.604 & 0.1 \\ 2010 / 03 / 31 & 17: 22: 12.639 & 33.183 & -115.599 & 0.1 \\ 2010 / 03 / 31 & 17: 54: 09.639 & 33.183 & -115.607 & 0.0 \\ 2010 / 03 / 31 & 17: 57: 57.639 & 33.179 & -115.594 & 1.5 \\ 2010 / 03 / 31 & 18: 13: 00.639 & 33.183 & -115.599 & 0.1 \\ 2010 / 03 / 31 & 21: 46: 53.639 & 33.117 & -115.613 & 7.9 \\ 2010 / 04 / 01 & 00: 41: 08.639 & 33.181 & -115.611 & 0.4 \\ 2010 / 04 / 01 & 00: 41: 57.639 & 33.189 & -115.635 & 3.2 \\ 2010 / 04 / 01 & 00: 46: 43.639 & 33.183 & -115.617 & 4.3 \\ 2010 / 04 / 01 & 00: 49: 03.639 & 33.186 & -115.612 & 0.6 \\ 2010 / 04 / 01 & 00: 54: 07.639 & 33.161 & -115.640 & 0.1 \\ 2010 / 04 / 01 & 01: 30: 18.639 & 33.165 & -115.623 & 7.0 \\ 2010 / 04 / 01 & 01: 31: 02.639 & 33.186 & -115.612 & 0.6 \\ 2010 / 04 / 01 & 01: 33: 45.639 & 33.193 & -115.615 & 3.9 \\ 2010 / 04 / 01 & 01: 40: 01.639 & 33.160 & -115.633 & 4.6 \\ 2010 / 04 / 01 & 01: 40: 19.639 & 33.100 & -115.590 & 14.6 \\ 2010 / 04 / 01 & 01: 42: 05.639 & 33.186 & -115.612 & 0.6 \\ 2010 / 04 / 01 & 01: 43: 01.639 & 33.188 & -115.599 & 0.8 \\ 2010 / 04 / 01 & 01: 45: 11.639 & 33.118 & -115.610 & 9.1 \\ 2010 / 04 / 01 & 01: 46: 04.639 & 33.118 & -115.610 & 9.1 \\ 2010 / 04 / 01 & 01: 46: 49.639 & 33.165 & -115.623 & 7.0 \\ 2010 / 04 / 01 & 01: 48: 07.639 & 33.161 & -115.640 & 0.1 \\ 2010 / 04 / 01 & 02: 33: 02.639 & 33.158 & -115.638 & 0.4\end{array}$




$\begin{array}{lllll}2010 / 04 / 01 & 02: 38: 08.639 & 33.181 & -115.611 & 0.4 \\ 2010 / 04 / 01 & 02: 49: 02.639 & 33.118 & -115.590 & 13.8 \\ 2010 / 04 / 01 & 02: 49: 19.639 & 33.176 & -115.609 & 2.1 \\ 2010 / 04 / 01 & 03: 02: 21.639 & 33.147 & -115.623 & 7.9 \\ 2010 / 04 / 01 & 03: 20: 32.639 & 33.118 & -115.610 & 9.1 \\ 2010 / 04 / 01 & 06: 05: 10.639 & 33.177 & -115.635 & 5.3 \\ 2010 / 04 / 01 & 09: 46: 09.639 & 33.174 & -115.603 & 0.1 \\ 2010 / 04 / 01 & 09: 46: 37.639 & 33.184 & -115.606 & 2.9 \\ 2010 / 04 / 01 & 10: 02: 39.639 & 33.181 & -115.665 & 4.2 \\ 2010 / 04 / 01 & 10: 04: 59.639 & 33.174 & -115.603 & 0.1 \\ 2010 / 04 / 01 & 12: 12: 03.639 & 33.176 & -115.604 & 0.0 \\ 2010 / 04 / 01 & 13: 25: 55.639 & 33.174 & -115.603 & 0.1 \\ 2010 / 04 / 01 & 14: 15: 52.639 & 33.176 & -115.609 & 2.1 \\ 2010 / 04 / 01 & 14: 33: 04.639 & 33.176 & -115.604 & 0.5 \\ 2010 / 04 / 01 & 14: 55: 37.639 & 33.185 & -115.569 & 3.7 \\ 2010 / 04 / 01 & 16: 23: 41.639 & 33.161 & -115.640 & 0.1 \\ 2010 / 04 / 02 & 02: 52: 15.639 & 33.199 & -115.570 & 0.2 \\ 2010 / 04 / 02 & 09: 08: 56.639 & 33.176 & -115.604 & 0.0 \\ 2010 / 04 / 02 & 11: 43: 32.639 & 33.192 & -115.572 & 1.6 \\ 2010 / 04 / 02 & 11: 48: 06.639 & 33.192 & -115.572 & 1.6 \\ 2010 / 04 / 02 & 13: 04: 54.639 & 33.184 & -115.606 & 2.9 \\ 2010 / 04 / 02 & 19: 50: 46.639 & 33.183 & -115.617 & 4.3 \\ 2010 / 04 / 03 & 00: 36: 37.639 & 33.176 & -115.604 & 0.0 \\ 2010 / 04 / 03 & 00: 40: 08.639 & 33.226 & -115.588 & 4.6 \\ 2010 / 04 / 03 & 07: 38: 47.639 & 33.177 & -115.635 & 5.3 \\ 2010 / 04 / 03 & 16: 44: 06.639 & 33.175 & -115.601 & 0.1 \\ 2010 / 04 / 03 & 20: 01: 07.639 & 33.181 & -115.659 & 6.0 \\ 2010 / 04 / 03 & 20: 03: 06.639 & 33.181 & -115.659 & 6.0 \\ 2010 / 04 / 03 & 20: 05: 28.639 & 33.177 & -115.606 & 0.1 \\ 2010 / 04 / 03 & 20: 12: 14.639 & 33.181 & -115.659 & 6.0 \\ 2010 / 04 / 03 & 21: 20: 28.639 & 33.184 & -115.606 & 2.9 \\ 2010 / 04 / 03 & 21: 55: 55.639 & 33.174 & -115.603 & 0.1 \\ 2010 / 04 / 03 & 22: 12: 45.639 & 33.173 & -115.650 & 1.8 \\ 2010 / 04 / 04 & 04: 48: 41.639 & 33.208 & -115.582 & 2.5 \\ 2010 / 04 / 04 & 12: 53: 48.639 & 33.179 & -115.597 & 0.1 \\ 2010 / 04 / 04 & 17: 15: 42.639 & 33.187 & -115.608 & 0.2 \\ 2010 / 04 / 04 & 18: 36: 53.639 & 33.174 & -115.603 & 0.1 \\ 2010 / 04 / 04 & 18: 41: 20.639 & 33.175 & -115.601 & 0.1 \\ 2010 / 04 / 04 & 23: 14: 54.639 & 33.100 & -115.590 & 14.6 \\ 2010 / 04 / 04 & 23: 21: 59.639 & 33.100 & -115.590 & 14.6 \\ 2010 / 04 / 04 & 23: 28: 50.639 & 33.184 & -115.597 & 0.1 \\ 2010 / 04 / 04 & 23: 29: 10.639 & 33.174 & -115.603 & 0.1 \\ 2010 / 04 / 05 & 00: 27: 26.639 & 33.176 & -115.604 & 0.0 \\ 2010 / 04 / 05 & 00: 41: 47.639 & 33.115 & -115.585 & 6.5 \\ 2010 / 04 / 05 & 00: 48: 45.639 & 33.100 & -115.590 & 14.6 \\ 2010 / 04 / 05 & 00: 55: 44.639 & 33.198 & -115.593 & 2.3\end{array}$




$\begin{array}{lllll}2010 / 04 / 05 & 02: 23: 42.639 & 33.165 & -115.623 & 7.0 \\ 2010 / 04 / 05 & 02: 28: 25.639 & 33.180 & -115.603 & 0.1 \\ 2010 / 04 / 05 & 02: 31: 23.639 & 33.117 & -115.613 & 7.9 \\ 2010 / 04 / 05 & 02: 46: 14.639 & 33.135 & -115.644 & 6.9 \\ 2010 / 04 / 05 & 03: 25: 44.639 & 33.175 & -115.600 & 1.1 \\ 2010 / 04 / 05 & 04: 20: 47.639 & 33.175 & -115.600 & 1.1 \\ 2010 / 04 / 05 & 04: 29: 41.639 & 33.187 & -115.604 & 3.2 \\ 2010 / 04 / 05 & 04: 34: 07.639 & 33.181 & -115.612 & 1.1 \\ 2010 / 04 / 05 & 04: 39: 33.639 & 33.218 & -115.639 & 3.0 \\ 2010 / 04 / 05 & 04: 53: 15.639 & 33.231 & -115.655 & 4.8 \\ 2010 / 04 / 05 & 06: 53: 09.639 & 33.175 & -115.606 & 4.2 \\ 2010 / 04 / 05 & 06: 54: 35.639 & 33.172 & -115.611 & 3.7 \\ 2010 / 04 / 05 & 07: 20: 48.639 & 33.218 & -115.639 & 3.0 \\ 2010 / 04 / 05 & 07: 52: 12.639 & 33.140 & -115.636 & 7.3 \\ 2010 / 04 / 05 & 07: 54: 47.639 & 33.208 & -115.631 & 5.0 \\ 2010 / 04 / 05 & 08: 05: 23.639 & 33.177 & -115.606 & 0.1 \\ 2010 / 04 / 05 & 12: 01: 16.639 & 33.142 & -115.628 & 10.3 \\ 2010 / 04 / 05 & 13: 05: 25.639 & 33.196 & -115.559 & 0.2 \\ 2010 / 04 / 05 & 13: 05: 55.639 & 33.175 & -115.601 & 0.1 \\ 2010 / 04 / 05 & 13: 15: 28.639 & 33.130 & -115.634 & 0.0 \\ 2010 / 04 / 05 & 13: 40: 11.639 & 33.130 & -115.634 & 0.0 \\ 2010 / 04 / 05 & 13: 43: 13.639 & 33.158 & -115.629 & 6.0 \\ 2010 / 04 / 05 & 14: 03: 00.639 & 33.130 & -115.634 & 0.0 \\ 2010 / 04 / 05 & 14: 25: 24.639 & 33.181 & -115.665 & 4.2 \\ 2010 / 04 / 05 & 14: 30: 05.639 & 33.108 & -115.602 & 8.1 \\ 2010 / 04 / 05 & 17: 48: 45.639 & 33.140 & -115.636 & 7.3 \\ 2010 / 04 / 05 & 20: 50: 41.639 & 33.208 & -115.631 & 5.0 \\ 2010 / 04 / 05 & 21: 36: 08.639 & 33.172 & -115.622 & 4.0 \\ 2010 / 04 / 05 & 21: 36: 32.639 & 33.208 & -115.631 & 5.0 \\ 2010 / 04 / 05 & 22: 12: 58.639 & 33.158 & -115.638 & 0.4 \\ 2010 / 04 / 05 & 22: 22: 01.639 & 33.176 & -115.618 & 3.7 \\ 2010 / 04 / 05 & 22: 23: 53.639 & 33.208 & -115.631 & 5.0 \\ 2010 / 04 / 05 & 22: 37: 44.639 & 33.173 & -115.650 & 1.8 \\ 2010 / 04 / 05 & 22: 42: 53.639 & 33.159 & -115.635 & 7.3 \\ 2010 / 04 / 06 & 01: 08: 59.639 & 33.142 & -115.628 & 10.3 \\ 2010 / 04 / 06 & 01: 28: 34.639 & 33.183 & -115.617 & 4.3 \\ 2010 / 04 / 06 & 01: 36: 49.639 & 33.208 & -115.631 & 5.0 \\ 2010 / 04 / 06 & 01: 40: 11.639 & 33.218 & -115.639 & 3.0 \\ 2010 / 04 / 06 & 01: 43: 58.639 & 33.181 & -115.603 & 2.0 \\ 2010 / 04 / 06 & 05: 19: 09.639 & 33.170 & -115.600 & 1.1 \\ 2010 / 04 / 06 & 06: 11: 47.639 & 33.208 & -115.631 & 5.0 \\ 2010 / 04 / 06 & 07: 07: 06.639 & 33.175 & -115.606 & 4.2 \\ 2010 / 04 / 06 & 07: 10: 41.639 & 33.218 & -115.639 & 3.0 \\ 2010 / 04 / 06 & 07: 53: 59.639 & 33.180 & -115.603 & 0.1\end{array}$




$\begin{array}{lllll}2010 / 04 / 06 & 08: 16: 27.639 & 33.175 & -115.601 & 0.1 \\ 2010 / 04 / 06 & 09: 11: 35.639 & 33.231 & -115.655 & 4.8 \\ 2010 / 04 / 06 & 09: 20: 10.639 & 33.208 & -115.631 & 5.0 \\ 2010 / 04 / 06 & 12: 00: 34.639 & 33.175 & -115.606 & 4.2 \\ 2010 / 04 / 06 & 12: 01: 12.639 & 33.149 & -115.640 & 3.5 \\ 2010 / 04 / 06 & 12: 11: 49.639 & 33.182 & -115.602 & 2.5 \\ 2010 / 04 / 06 & 12: 16: 11.639 & 33.181 & -115.611 & 0.4 \\ 2010 / 04 / 06 & 12: 21: 50.639 & 33.108 & -115.602 & 8.1 \\ 2010 / 04 / 06 & 13: 36: 36.639 & 33.175 & -115.610 & 2.4 \\ 2010 / 04 / 06 & 14: 23: 25.639 & 33.174 & -115.603 & 0.1 \\ 2010 / 04 / 06 & 14: 40: 40.639 & 33.174 & -115.603 & 0.1 \\ 2010 / 04 / 06 & 14: 41: 34.639 & 33.186 & -115.612 & 0.6 \\ 2010 / 04 / 06 & 15: 23: 07.639 & 33.174 & -115.603 & 0.1 \\ 2010 / 04 / 06 & 15: 28: 35.639 & 33.176 & -115.604 & 0.5 \\ 2010 / 04 / 06 & 15: 29: 43.639 & 33.228 & -115.626 & 3.7 \\ 2010 / 04 / 06 & 15: 37: 51.639 & 33.184 & -115.606 & 2.9 \\ 2010 / 04 / 06 & 17: 38: 34.639 & 33.174 & -115.603 & 0.1 \\ 2010 / 04 / 06 & 19: 15: 54.639 & 33.140 & -115.636 & 7.3 \\ 2010 / 04 / 06 & 19: 22: 31.639 & 33.181 & -115.639 & 3.7 \\ 2010 / 04 / 06 & 19: 25: 01.639 & 33.165 & -115.623 & 7.0 \\ 2010 / 04 / 06 & 19: 25: 16.639 & 33.208 & -115.631 & 5.0 \\ 2010 / 04 / 06 & 19: 46: 23.639 & 33.208 & -115.631 & 5.0 \\ 2010 / 04 / 06 & 21: 39: 53.639 & 33.184 & -115.606 & 2.9 \\ 2010 / 04 / 06 & 22: 26: 17.639 & 33.181 & -115.639 & 3.7 \\ 2010 / 04 / 06 & 22: 46: 59.639 & 33.177 & -115.606 & 0.1 \\ 2010 / 04 / 06 & 22: 47: 44.639 & 33.174 & -115.603 & 0.1 \\ 2010 / 04 / 07 & 00: 11: 36.639 & 33.213 & -115.584 & 1.3 \\ 2010 / 04 / 07 & 00: 13: 28.639 & 33.231 & -115.657 & 2.0 \\ 2010 / 04 / 07 & 01: 42: 47.639 & 33.231 & -115.657 & 2.0 \\ 2010 / 04 / 07 & 01: 58: 38.639 & 33.208 & -115.631 & 5.0 \\ 2010 / 04 / 07 & 02: 37: 32.639 & 33.228 & -115.626 & 3.7 \\ 2010 / 04 / 07 & 03: 00: 19.639 & 33.182 & -115.602 & 2.5 \\ 2010 / 04 / 07 & 03: 01: 11.639 & 33.193 & -115.615 & 3.9 \\ 2010 / 04 / 07 & 03: 25: 45.639 & 33.193 & -115.615 & 3.9 \\ 2010 / 04 / 07 & 03: 25: 58.639 & 33.193 & -115.615 & 3.9 \\ 2010 / 04 / 07 & 03: 42: 46.639 & 33.176 & -115.608 & 0.4 \\ 2010 / 04 / 07 & 03: 49: 53.639 & 33.176 & -115.604 & 0.0 \\ 2010 / 04 / 07 & 04: 25: 29.639 & 33.193 & -115.615 & 3.9 \\ 2010 / 04 / 07 & 05: 40: 31.639 & 33.226 & -115.588 & 4.6 \\ 2010 / 04 / 07 & 09: 23: 18.639 & 33.226 & -115.610 & 2.4 \\ 2010 / 04 / 07 & 12: 40: 53.639 & 33.226 & -115.588 & 4.6 \\ 2010 / 04 / 07 & 12: 55: 57.639 & 33.174 & -115.603 & 0.1 \\ 207 & 13: 59: 41.639 & 33.174 & -115.603 & 0.1 \\ 20.20 .639 & 33.175 & -115.610 & 2.4\end{array}$




\begin{tabular}{lllll}
$2010 / 04 / 07$ & $13: 05: 33.639$ & 33.196 & -115.587 & 5.3 \\
$2010 / 04 / 07$ & $13: 19: 29.639$ & 33.183 & -115.607 & 0.0 \\
$2010 / 04 / 07$ & $14: 12: 20.639$ & 33.201 & -115.544 & 3.4 \\
$2010 / 04 / 07$ & $15: 21: 32.639$ & 33.181 & -115.639 & 3.7 \\
$2010 / 04 / 07$ & $15: 48: 07.639$ & 33.177 & -115.635 & 5.3 \\
$2010 / 04 / 07$ & $17: 35: 18.639$ & 33.181 & -115.639 & 3.7 \\
$2010 / 04 / 07$ & $20: 40: 25.639$ & 33.231 & -115.655 & 4.8 \\
$2010 / 04 / 07$ & $21: 23: 08.639$ & 33.231 & -115.655 & 4.8 \\
$2010 / 04 / 07$ & $22: 35: 12.639$ & 33.176 & -115.604 & 0.5 \\
$2010 / 04 / 07$ & $22: 57: 45.639$ & 33.181 & -115.639 & 3.7 \\
$2010 / 04 / 08$ & $00: 13: 20.639$ & 33.190 & -115.574 & 3.1 \\
$2010 / 04 / 08$ & $00: 46: 14.639$ & 33.181 & -115.639 & 3.7 \\
$2010 / 04 / 08$ & $02: 09: 40.639$ & 33.181 & -115.639 & 3.7 \\
$2010 / 04 / 08$ & $04: 51: 48.639$ & 33.218 & -115.639 & 3.0 \\
$2010 / 04 / 08$ & $05: 05: 10.639$ & 33.201 & -115.544 & 3.4 \\
$2010 / 04 / 08$ & $06: 38: 07.639$ & 33.181 & -115.639 & 3.7 \\
$2010 / 04 / 08$ & $10: 05: 59.639$ & 33.177 & -115.606 & 0.1 \\
$2010 / 04 / 08$ & $17: 03: 20.639$ & 33.183 & -115.617 & 4.3 \\
$2010 / 04 / 08$ & $17: 37: 29.639$ & 33.184 & -115.608 & 0.3 \\
$2010 / 04 / 08$ & $18: 42: 16.639$ & 33.193 & -115.615 & 3.9 \\
$2010 / 04 / 08$ & $19: 04: 02.639$ & 33.193 & -115.615 & 3.9 \\
$2010 / 04 / 08$ & $21: 16: 41.639$ & 33.228 & -115.626 & 3.7 \\
$2010 / 04 / 08$ & $22: 06: 24.639$ & 33.177 & -115.635 & 5.3 \\
$2010 / 04 / 09$ & $00: 02: 29.639$ & 33.228 & -115.626 & 3.7 \\
$2010 / 04 / 09$ & $00: 24: 00.639$ & 33.176 & -115.604 & 0.0 \\
$2010 / 04 / 09$ & $05: 52: 31.639$ & 33.183 & -115.617 & 4.3 \\
$2010 / 04 / 09$ & $07: 49: 17.639$ & 33.231 & -115.655 & 4.8 \\
$2010 / 04 / 09$ & $07: 56: 13.639$ & 33.182 & -115.603 & 0.1 \\
$2010 / 04 / 09$ & $08: 09: 52.639$ & 33.149 & -115.640 & 3.5 \\
$2010 / 04 / 09$ & $09: 04: 32.639$ & 33.176 & -115.609 & 2.1 \\
$2010 / 04 / 09$ & $10: 55: 33.639$ & 33.184 & -115.604 & 0.1 \\
$2010 / 04 / 09$ & $11: 00: 21.639$ & 33.182 & -115.603 & 0.1 \\
$2010 / 04 / 09$ & $12: 25: 54.639$ & 33.231 & -115.657 & 2.0 \\
$2010 / 04 / 09$ & $13: 04: 37.639$ & 33.142 & -115.628 & 10.3 \\
$2010 / 04 / 09$ & $14: 56: 18.639$ & 33.228 & -115.626 & 3.7 \\
$2010 / 04 / 09$ & $22: 09: 02.639$ & 33.181 & -115.603 & 1.4 \\
$2010 / 04 / 09$ & $22: 53: 14.639$ & 33.231 & -115.655 & 4.8 \\
$2010 / 04 / 10$ & $01: 49: 20.639$ & 33.208 & -115.631 & 5.0 \\
$2010 / 04 / 10$ & $05: 26: 47.639$ & 33.231 & -115.657 & 2.0 \\
$2010 / 04 / 10$ & $08: 19: 36.639$ & 33.179 & -115.594 & 1.5 \\
$2010 / 04 / 10$ & $08: 23: 17.639$ & 33.180 & -115.600 & 0.1 \\
$2010 / 04 / 10$ & $10: 24: 47.639$ & 33.176 & -115.618 & 3.7 \\
$2010 / 04 / 10$ & $11: 57: 21.639$ & 33.241 & -115.633 & 3.4 \\
$2010 / 04 / 11$ & $02: 05: 52.639$ & 33.185 & -115.608 & 2.0 \\
$204 / 11$ & $02: 19: 07.639$ & 33.185 & -115.608 & 2.0 \\
\hline $2: 45: 39.639$ & 33.208 & -115.631 & 5.0
\end{tabular}




$\begin{array}{lllll}2010 / 04 / 11 & 10: 02: 22.639 & 33.135 & -115.644 & 6.9 \\ 2010 / 04 / 12 & 03: 02: 11.639 & 33.172 & -115.611 & 3.7 \\ 2010 / 04 / 12 & 05: 17: 40.639 & 33.241 & -115.633 & 3.4 \\ 2010 / 04 / 12 & 15: 10: 35.639 & 33.179 & -115.614 & 1.8 \\ 2010 / 04 / 12 & 17: 04: 08.639 & 33.214 & -115.620 & 5.3 \\ 2010 / 04 / 12 & 19: 47: 00.639 & 33.229 & -115.576 & 3.4 \\ 2010 / 04 / 12 & 19: 53: 49.639 & 33.185 & -115.569 & 3.7 \\ 2010 / 04 / 12 & 19: 55: 46.639 & 33.179 & -115.594 & 1.5 \\ 2010 / 04 / 12 & 19: 56: 06.639 & 33.229 & -115.576 & 3.4 \\ 2010 / 04 / 12 & 19: 59: 00.639 & 33.189 & -115.605 & 1.5 \\ 2010 / 04 / 13 & 00: 36: 26.639 & 33.181 & -115.608 & 1.0 \\ 2010 / 04 / 13 & 00: 51: 08.639 & 33.176 & -115.604 & 0.5 \\ 2010 / 04 / 13 & 03: 21: 53.639 & 33.201 & -115.544 & 3.4 \\ 2010 / 04 / 13 & 05: 17: 23.639 & 33.181 & -115.612 & 1.1 \\ 2010 / 04 / 13 & 09: 30: 59.639 & 33.135 & -115.644 & 6.9 \\ 2010 / 04 / 13 & 11: 05: 21.639 & 33.198 & -115.571 & 0.2 \\ 2010 / 04 / 13 & 13: 22: 09.639 & 33.188 & -115.600 & 0.1 \\ 2010 / 04 / 13 & 18: 08: 19.639 & 33.176 & -115.608 & 0.4 \\ 2010 / 04 / 13 & 20: 03: 23.639 & 33.188 & -115.571 & 3.0 \\ 2010 / 04 / 14 & 00: 14: 26.639 & 33.190 & -115.604 & 1.2 \\ 2010 / 04 / 21 & 00: 01: 41.639 & 33.180 & -115.600 & 0.1 \\ 2010 / 04 / 21 & 00: 02: 11.639 & 33.165 & -115.623 & 7.0 \\ 2010 / 04 / 21 & 00: 34: 37.639 & 33.176 & -115.608 & 0.4 \\ 2010 / 04 / 21 & 00: 38: 08.639 & 33.176 & -115.608 & 0.4 \\ 2010 / 04 / 21 & 05: 18: 48.639 & 33.176 & -115.608 & 0.4 \\ 2010 / 04 / 21 & 09: 42: 23.639 & 33.176 & -115.609 & 2.1 \\ 2010 / 04 / 21 & 15: 36: 40.639 & 33.192 & -115.572 & 1.6 \\ 2010 / 04 / 22 & 00: 36: 31.639 & 33.182 & -115.618 & 3.1 \\ 2010 / 04 / 22 & 00: 49: 35.639 & 33.187 & -115.608 & 0.2 \\ 2010 / 04 / 22 & 01: 52: 08.639 & 33.184 & -115.601 & 3.5 \\ 2010 / 04 / 22 & 02: 18: 31.639 & 33.177 & -115.621 & 2.7 \\ 2010 / 04 / 22 & 05: 52: 26.639 & 33.196 & -115.587 & 5.3 \\ 2010 / 04 / 22 & 05: 54: 00.639 & 33.196 & -115.587 & 5.3 \\ 2010 / 04 / 22 & 06: 23: 33.639 & 33.208 & -115.582 & 2.5 \\ 2010 / 04 / 22 & 09: 34: 09.639 & 33.208 & -115.582 & 2.5 \\ 2010 / 04 / 22 & 12: 12: 07.639 & 33.183 & -115.599 & 0.1 \\ 2010 / 04 / 22 & 14: 25: 57.639 & 33.177 & -115.606 & 0.1 \\ 2010 / 04 / 22 & 21: 40: 25.639 & 33.130 & -115.634 & 0.0 \\ 2010 / 04 / 22 & 21: 44: 20.639 & 33.176 & -115.604 & 0.5 \\ 2010 / 04 / 22 & 21: 49: 37.639 & 33.179 & -115.594 & 1.5 \\ 2010 / 04 / 22 & 23: 06: 16.639 & 33.183 & -115.599 & 0.1 \\ 2010 / 04 / 31.639 & 33.181 & -115.608 & 1.0 \\ 23 / 04 / 22 & 23: 13: 47.639 & 33.183 & -115.599 & 0.1 \\ 23: 21: 26.639 & 33.208 & -115.582 & 2.5\end{array}$




$\begin{array}{lllll}2010 / 04 / 22 & 23: 31: 02.639 & 33.183 & -115.599 & 0.1 \\ 2010 / 04 / 22 & 23: 36: 04.639 & 33.183 & -115.599 & 0.1 \\ 2010 / 04 / 22 & 23: 46: 34.639 & 33.181 & -115.659 & 6.0 \\ 2010 / 04 / 22 & 23: 52: 18.639 & 33.176 & -115.618 & 3.7 \\ 2010 / 04 / 23 & 00: 38: 43.639 & 33.177 & -115.606 & 0.1 \\ 2010 / 04 / 23 & 01: 55: 57.639 & 33.201 & -115.544 & 3.4 \\ 2010 / 04 / 23 & 02: 06: 33.639 & 33.184 & -115.604 & 0.1 \\ 2010 / 04 / 23 & 02: 16: 23.639 & 33.184 & -115.611 & 2.7 \\ 2010 / 04 / 23 & 02: 17: 12.639 & 33.187 & -115.608 & 0.2 \\ 2010 / 04 / 23 & 02: 19: 07.639 & 33.184 & -115.604 & 0.1 \\ 2010 / 04 / 23 & 02: 19: 46.639 & 33.181 & -115.665 & 4.2 \\ 2010 / 04 / 23 & 02: 19: 59.639 & 33.184 & -115.604 & 0.1 \\ 2010 / 04 / 23 & 02: 20: 26.639 & 33.184 & -115.604 & 0.1 \\ 2010 / 04 / 23 & 02: 21: 20.639 & 33.181 & -115.665 & 4.2 \\ 2010 / 04 / 23 & 02: 21: 52.639 & 33.184 & -115.604 & 0.1 \\ 2010 / 04 / 23 & 02: 22: 20.639 & 33.181 & -115.665 & 4.2 \\ 2010 / 04 / 23 & 02: 22: 55.639 & 33.181 & -115.665 & 4.2 \\ 2010 / 04 / 23 & 02: 24: 10.639 & 33.181 & -115.665 & 4.2 \\ 2010 / 04 / 23 & 02: 25: 05.639 & 33.184 & -115.604 & 0.1 \\ 2010 / 04 / 23 & 02: 25: 49.639 & 33.181 & -115.665 & 4.2 \\ 2010 / 04 / 23 & 02: 26: 22.639 & 33.214 & -115.620 & 5.3 \\ 2010 / 04 / 23 & 02: 29: 26.639 & 33.214 & -115.620 & 5.3 \\ 2010 / 04 / 23 & 02: 29: 41.639 & 33.180 & -115.603 & 0.1 \\ 2010 / 04 / 23 & 02: 30: 13.639 & 33.214 & -115.620 & 5.3 \\ 2010 / 04 / 23 & 02: 31: 34.639 & 33.181 & -115.665 & 4.2 \\ 2010 / 04 / 23 & 02: 35: 06.639 & 33.181 & -115.665 & 4.2 \\ 2010 / 04 / 23 & 02: 37: 39.639 & 33.176 & -115.609 & 2.1 \\ 2010 / 04 / 23 & 02: 38: 17.639 & 33.179 & -115.614 & 1.8 \\ 2010 / 04 / 23 & 02: 40: 43.639 & 33.176 & -115.609 & 2.1 \\ 2010 / 04 / 23 & 02: 41: 28.639 & 33.181 & -115.659 & 6.0 \\ 2010 / 04 / 23 & 02: 53: 17.639 & 33.165 & -115.623 & 7.0 \\ 2010 / 04 / 23 & 03: 01: 46.639 & 33.189 & -115.619 & 2.2 \\ 2010 / 04 / 23 & 03: 04: 30.639 & 33.183 & -115.617 & 4.3 \\ 2010 / 04 / 23 & 03: 06: 31.639 & 33.179 & -115.594 & 1.5 \\ 2010 / 04 / 23 & 03: 07: 04.639 & 33.186 & -115.612 & 0.6 \\ 2010 / 04 / 23 & 03: 09: 02.639 & 33.184 & -115.610 & 2.8 \\ 2010 / 04 / 23 & 03: 09: 34.639 & 33.179 & -115.614 & 1.8 \\ 2010 / 04 / 23 & 03: 20: 11.639 & 33.189 & -115.619 & 2.2 \\ 2010 / 04 / 23 & 03: 27: 30.639 & 33.176 & -115.604 & 0.5 \\ 2010 / 04 / 23 & 03: 37: 15.639 & 33.184 & -115.611 & 2.7 \\ 2010 / 04 / 23 & 03: 39: 07.639 & 33.184 & -115.604 & 0.1 \\ 2010 / 04 / 23 & 03: 52: 05.639 & 33.183 & -115.599 & 0.1 \\ 2010 / 04 / 23 & 03: 53: 06.639 & 33.184 & -115.604 & 0.1 \\ 2010 / 04 / 23 & 04: 14: 47.639 & 33.193 & -115.604 & 0.1 \\ & 04: 25: 04.639 & 33.181 & -115.611 & 0.4\end{array}$




\begin{tabular}{|c|c|c|c|}
\hline $2010 / 04 / 23$ & $04: 26: 02.639$ & 33.176 & -115.608 \\
\hline $2010 / 04 / 23$ & $04: 26: 27.639$ & 33.186 & -115.612 \\
\hline $2010 / 04 / 23$ & $04: 34: 37.639$ & 33.184 & -115.608 \\
\hline $2010 / 04 / 23$ & $04: 36: 28.639$ & 33.192 & -115.572 \\
\hline $2010 / 04 / 23$ & $05: 03: 28.639$ & 33.186 & -115.612 \\
\hline $2010 / 04 / 23$ & 05:13:08.639 & 33.184 & -115.604 \\
\hline $2010 / 04 / 23$ & $05: 33: 30.639$ & 33.184 & -115.609 \\
\hline $2010 / 04 / 23$ & $05: 33: 55.639$ & 33.184 & -115.609 \\
\hline $2010 / 04 / 23$ & 05:37:07.639 & 33.179 & -115.594 \\
\hline $2010 / 04 / 23$ & $05: 41: 16.639$ & 33.181 & -115.665 \\
\hline $2010 / 04 / 23$ & $05: 41: 37.639$ & 33.176 & -115.604 \\
\hline $2010 / 04 / 23$ & $05: 45: 35.639$ & 33.183 & -115.606 \\
\hline $2010 / 04 / 23$ & $06: 56: 46.639$ & 33.193 & -115.583 \\
\hline $2010 / 04 / 23$ & $10: 31: 57.639$ & 33.221 & -115.605 \\
\hline $2010 / 04 / 23$ & $10: 32: 31.639$ & 33.221 & -115.605 \\
\hline $2010 / 04 / 23$ & $13: 01: 00.639$ & 33.236 & -115.573 \\
\hline $2010 / 04 / 23$ & $13: 31: 34.639$ & 33.208 & -115.582 \\
\hline $2010 / 04 / 24$ & $17: 31: 50.639$ & 33.186 & -115.593 \\
\hline $2010 / 04 / 25$ & $03: 30: 55.639$ & 33.176 & -115.608 \\
\hline $2010 / 04 / 25$ & 05:30:06.639 & 33.201 & -115.544 \\
\hline $2010 / 04 / 25$ & $05: 58: 16.639$ & 33.183 & -115.599 \\
\hline $2010 / 04 / 25$ & $07: 43: 28.639$ & 33.170 & -115.600 \\
\hline $2010 / 04 / 25$ & $08: 05: 24.639$ & 33.179 & -115.594 \\
\hline $2010 / 04 / 25$ & $18: 31: 38.639$ & 33.173 & -115.650 \\
\hline $2010 / 04 / 25$ & $18: 47: 48.639$ & 33.181 & -115.608 \\
\hline $2010 / 04 / 25$ & $21: 40: 08.639$ & 33.184 & -115.601 \\
\hline $2010 / 04 / 25$ & $21: 50: 32.639$ & 33.181 & -115.614 \\
\hline $2010 / 04 / 26$ & $00: 44: 17.639$ & 33.231 & -115.655 \\
\hline $2010 / 04 / 26$ & $01: 55: 36.639$ & 33.176 & -115.609 \\
\hline $2010 / 04 / 26$ & 02:26:06.639 & 33.145 & -115.634 \\
\hline $2010 / 04 / 26$ & $02: 28: 23.639$ & 33.118 & -115.610 \\
\hline $2010 / 04 / 26$ & $06: 28: 55.639$ & 33.234 & -115.604 \\
\hline $2010 / 04 / 26$ & $07: 31: 17.639$ & 33.179 & -115.594 \\
\hline $2010 / 04 / 26$ & $07: 42: 54.639$ & 33.181 & -115.612 \\
\hline $2010 / 04 / 26$ & $10: 22: 03.639$ & 33.188 & -115.599 \\
\hline $2010 / 04 / 26$ & $10: 22: 16.639$ & 33.188 & -115.599 \\
\hline $2010 / 04 / 26$ & $10: 57: 44.639$ & 33.188 & -115.599 \\
\hline $2010 / 04 / 26$ & $13: 00: 25.639$ & 33.170 & -115.600 \\
\hline $2010 / 04 / 26$ & $14: 58: 35.639$ & 33.196 & -115.587 \\
\hline $2010 / 04 / 26$ & $17: 05: 11.639$ & 33.176 & -115.609 \\
\hline $2010 / 04 / 26$ & $17: 14: 21.639$ & 33.214 & -115.620 \\
\hline $2010 / 04 / 26$ & $17: 19: 52.639$ & 33.201 & -115.544 \\
\hline $2010 / 04 / 26$ & $18: 41: 13.639$ & 33.181 & -115.665 \\
\hline $2010 / 04 / 26$ & $19: 21: 55.639$ & 33.193 & -115.615 \\
\hline $2010 / 04 / 27$ & $04: 44: 37.579$ & 33.183 & -115.607 \\
\hline $2010 / 04 / 27$ & $04: 44: 54.579$ & 33.197 & -115.596 \\
\hline
\end{tabular}




$\begin{array}{lllll}2010 / 04 / 27 & 07: 10: 36.579 & 33.179 & -115.594 & 1.5 \\ 2010 / 04 / 27 & 07: 11: 10.579 & 33.165 & -115.623 & 7.0 \\ 2010 / 04 / 27 & 22: 03: 40.579 & 33.172 & -115.611 & 3.7 \\ 2010 / 04 / 27 & 22: 48: 29.579 & 33.208 & -115.582 & 2.5 \\ 2010 / 04 / 27 & 22: 49: 10.579 & 33.180 & -115.600 & 0.1 \\ 2010 / 04 / 28 & 02: 33: 29.579 & 33.184 & -115.609 & 2.2 \\ 2010 / 04 / 28 & 05: 13: 33.579 & 33.188 & -115.609 & 2.3 \\ 2010 / 04 / 28 & 05: 41: 59.579 & 33.181 & -115.639 & 3.7 \\ 2010 / 04 / 28 & 05: 55: 43.579 & 33.182 & -115.603 & 0.1 \\ 2010 / 04 / 28 & 06: 16: 15.579 & 33.176 & -115.608 & 0.4 \\ 2010 / 04 / 28 & 06: 43: 40.579 & 33.187 & -115.608 & 0.2 \\ 2010 / 04 / 28 & 07: 27: 11.579 & 33.142 & -115.628 & 10.3 \\ 2010 / 04 / 28 & 07: 53: 46.579 & 33.181 & -115.639 & 3.7 \\ 2010 / 04 / 28 & 11: 52: 05.579 & 33.181 & -115.603 & 2.0 \\ 2010 / 04 / 28 & 12: 00: 29.579 & 33.181 & -115.603 & 2.0 \\ 2010 / 04 / 28 & 12: 00: 44.579 & 33.181 & -115.603 & 2.0 \\ 2010 / 04 / 28 & 12: 01: 13.579 & 33.184 & -115.604 & 0.1 \\ 2010 / 04 / 28 & 12: 02: 20.579 & 33.181 & -115.603 & 2.0 \\ 2010 / 04 / 28 & 12: 03: 35.579 & 33.181 & -115.603 & 2.0 \\ 2010 / 04 / 28 & 12: 08: 43.579 & 33.188 & -115.609 & 2.3 \\ 2010 / 04 / 28 & 12: 31: 14.579 & 33.229 & -115.645 & 5.7 \\ 2010 / 04 / 28 & 12: 58: 10.579 & 33.184 & -115.611 & 2.7 \\ 2010 / 04 / 28 & 13: 28: 54.579 & 33.184 & -115.609 & 2.2 \\ 2010 / 04 / 28 & 13: 29: 06.579 & 33.184 & -115.608 & 0.3 \\ 2010 / 04 / 28 & 13: 45: 36.579 & 33.188 & -115.609 & 2.3 \\ 2010 / 04 / 29 & 03: 57: 50.579 & 33.183 & -115.599 & 0.1 \\ 2010 / 04 / 29 & 05: 31: 54.579 & 33.164 & -115.640 & 3.4 \\ 2010 / 04 / 29 & 09: 13: 24.579 & 33.181 & -115.614 & 3.1 \\ 2010 / 04 / 29 & 11: 30: 03.579 & 33.208 & -115.631 & 5.0 \\ 2010 / 04 / 29 & 15: 46: 16.579 & 33.176 & -115.604 & 0.5 \\ 2010 / 04 / 29 & 17: 09: 39.579 & 33.170 & -115.600 & 1.1 \\ 2010 / 04 / 30 & 02: 54: 08.639 & 33.202 & -115.575 & 3.8 \\ 2010 / 04 / 30 & 03: 50: 10.639 & 33.176 & -115.608 & 0.4 \\ 2010 / 04 / 30 & 03: 54: 11.639 & 33.183 & -115.606 & 0.1 \\ 2010 / 04 / 30 & 03: 57: 30.639 & 33.202 & -115.671 & 6.3 \\ 2010 / 04 / 30 & 16: 37: 26.639 & 33.186 & -115.614 & 2.4 \\ 2010 / 04 / 30 & 18: 55: 19.639 & 33.170 & -115.600 & 1.1 \\ 2010 / 04 / 30 & 19: 56: 32.639 & 33.196 & -115.559 & 0.2 \\ 2010 / 05 / 01 & 03: 11: 41.579 & 33.177 & -115.606 & 0.1 \\ 2010 / 05 / 01 & 17: 32: 53.579 & 33.229 & -115.576 & 3.4 \\ 2010 / 05 / 01 & 22: 34: 41.579 & 33.118 & -115.610 & 9.1 \\ 2010 / 05 / 02 & 00: 19: 45.639 & 33.203 & -115.579 & 3.5 \\ 2010 / 05 / 02 & 05: 45: 05.639 & 33.214 & -115.620 & 5.3 \\ 2010 / 05 / 02 & 12: 58: 58.639 & 33.184 & -115.611 & 2.7 \\ 2010 / 05 / 02 & 13: 00: 05.639 & 33.187 & -115.608 & 0.2\end{array}$




$\begin{array}{lllll}2010 / 05 / 02 & 13: 02: 30.639 & 33.179 & -115.614 & 1.8 \\ 2010 / 05 / 02 & 14: 03: 43.639 & 33.231 & -115.655 & 4.8 \\ 2010 / 05 / 02 & 19: 07: 34.639 & 33.184 & -115.606 & 2.9 \\ 2010 / 05 / 02 & 20: 02: 42.639 & 33.196 & -115.587 & 5.3 \\ 2010 / 05 / 03 & 13: 43: 32.639 & 33.168 & -115.609 & 0.7 \\ 2010 / 05 / 03 & 15: 38: 34.639 & 33.157 & -115.680 & 0.2 \\ 2010 / 05 / 03 & 15: 54: 33.639 & 33.190 & -115.608 & 2.2 \\ 2010 / 05 / 03 & 15: 58: 03.639 & 33.176 & -115.608 & 0.4 \\ 2010 / 05 / 03 & 15: 59: 04.639 & 33.188 & -115.599 & 0.8 \\ 2010 / 05 / 03 & 22: 39: 48.639 & 33.231 & -115.655 & 4.8 \\ 2010 / 05 / 04 & 02: 29: 25.639 & 33.190 & -115.608 & 2.2 \\ 2010 / 05 / 04 & 08: 34: 45.639 & 33.183 & -115.607 & 0.0 \\ 2010 / 05 / 04 & 08: 35: 28.639 & 33.183 & -115.607 & 0.0 \\ 2010 / 05 / 04 & 08: 47: 30.639 & 33.176 & -115.608 & 0.4 \\ 2010 / 05 / 04 & 08: 56: 20.639 & 33.175 & -115.601 & 0.1 \\ 2010 / 05 / 04 & 08: 56: 59.639 & 33.176 & -115.608 & 0.4 \\ 2010 / 05 / 04 & 10: 07: 17.639 & 33.177 & -115.614 & 1.2 \\ 2010 / 05 / 04 & 10: 32: 43.639 & 33.177 & -115.614 & 1.2 \\ 2010 / 05 / 04 & 10: 33: 45.639 & 33.177 & -115.614 & 1.2 \\ 2010 / 05 / 04 & 15: 44: 30.639 & 33.192 & -115.572 & 1.6 \\ 2010 / 05 / 04 & 20: 54: 40.639 & 33.192 & -115.626 & 7.7 \\ 2010 / 05 / 04 & 20: 57: 28.639 & 33.192 & -115.626 & 7.7 \\ 2010 / 05 / 04 & 20: 58: 00.639 & 33.192 & -115.626 & 7.7 \\ 2010 / 05 / 04 & 21: 35: 25.639 & 33.179 & -115.621 & 4.8 \\ 2010 / 05 / 08 & 09: 22: 56.579 & 33.118 & -115.610 & 9.1 \\ 2010 / 05 / 08 & 16: 52: 26.579 & 33.168 & -115.609 & 0.7 \\ 2010 / 05 / 08 & 17: 16: 00.579 & 33.194 & -115.583 & 3.0 \\ 2010 / 05 / 09 & 15: 06: 19.579 & 33.130 & -115.634 & 0.0 \\ 2010 / 05 / 10 & 04: 47: 20.579 & 33.177 & -115.606 & 0.1 \\ 2010 / 05 / 10 & 04: 50: 43.579 & 33.176 & -115.608 & 0.4 \\ 2010 / 05 / 10 & 04: 51: 29.579 & 33.179 & -115.594 & 1.5 \\ 2010 / 05 / 10 & 04: 51: 50.579 & 33.182 & -115.602 & 2.5 \\ 2010 / 05 / 10 & 04: 52: 29.579 & 33.176 & -115.608 & 0.4 \\ 2010 / 05 / 10 & 09: 01: 52.579 & 33.186 & -115.612 & 0.6 \\ 2010 / 05 / 10 & 09: 06: 30.579 & 33.186 & -115.612 & 0.6 \\ 2010 / 05 / 10 & 09: 09: 42.579 & 33.187 & -115.604 & 3.2 \\ 2010 / 05 / 10 & 12: 23: 08.579 & 33.181 & -115.602 & 0.4 \\ 2010 / 05 / 10 & 17: 54: 36.579 & 33.193 & -115.583 & 5.9 \\ 2010 / 05 / 10 & 17: 56: 19.579 & 33.187 & -115.608 & 0.2 \\ 2010 / 05 / 10 & 17: 56: 45.579 & 33.176 & -115.608 & 0.4 \\ 2010 / 05 / 10 & 17: 57: 02.579 & 33.193 & -115.615 & 3.9 \\ 2010 / 05 / 18 & 00: 44: 59.579 & 33.203 & -115.626 & 7.7 \\ 2010 / 05 / 19 & 07: 18: 09.639 & 33.185 & -115.569 & 3.7 \\ 205 / 20 & 07: 47: 15.639 & 33.201 & -115.544 & 3.4 \\ 05: 42: 05.639 & 33.177 & -115.621 & 2.7\end{array}$




$\begin{array}{lllll}2010 / 05 / 20 & 16: 00: 00.639 & 33.197 & -115.596 & 3.0 \\ 2010 / 05 / 20 & 16: 01: 34.639 & 33.197 & -115.596 & 3.0 \\ 2010 / 05 / 20 & 16: 14: 35.639 & 33.181 & -115.608 & 1.0 \\ 2010 / 05 / 20 & 16: 21: 40.639 & 33.176 & -115.608 & 0.4 \\ 2010 / 05 / 20 & 16: 23: 24.639 & 33.181 & -115.611 & 0.4 \\ 2010 / 05 / 20 & 16: 24: 52.639 & 33.176 & -115.608 & 0.4 \\ 2010 / 05 / 20 & 16: 25: 58.639 & 33.214 & -115.620 & 5.3 \\ 2010 / 05 / 20 & 16: 47: 52.639 & 33.176 & -115.608 & 0.4 \\ 2010 / 05 / 20 & 19: 21: 50.639 & 33.176 & -115.608 & 0.4 \\ 2010 / 05 / 20 & 19: 28: 52.639 & 33.182 & -115.603 & 0.1 \\ 2010 / 05 / 20 & 19: 40: 18.639 & 33.181 & -115.665 & 4.2 \\ 2010 / 05 / 20 & 19: 55: 09.639 & 33.176 & -115.608 & 0.4 \\ 2010 / 05 / 20 & 20: 04: 58.639 & 33.183 & -115.607 & 0.0 \\ 2010 / 05 / 20 & 20: 21: 17.639 & 33.214 & -115.620 & 5.3 \\ 2010 / 05 / 20 & 21: 17: 39.639 & 33.212 & -115.633 & 7.6 \\ 2010 / 05 / 20 & 23: 07: 45.639 & 33.188 & -115.609 & 2.3 \\ 2010 / 05 / 21 & 05: 40: 02.579 & 33.188 & -115.599 & 0.8 \\ 2010 / 05 / 21 & 11: 04: 29.579 & 33.173 & -115.609 & 0.1 \\ 2010 / 05 / 21 & 11: 05: 39.579 & 33.176 & -115.609 & 2.1 \\ 2010 / 05 / 21 & 11: 11: 40.579 & 33.176 & -115.609 & 2.1 \\ 2010 / 05 / 21 & 11: 37: 22.579 & 33.176 & -115.609 & 2.1 \\ 2010 / 05 / 21 & 11: 45: 55.579 & 33.214 & -115.620 & 5.3 \\ 2010 / 05 / 21 & 11: 47: 49.579 & 33.176 & -115.608 & 0.4 \\ 2010 / 05 / 21 & 11: 49: 12.579 & 33.182 & -115.603 & 0.1 \\ 2010 / 05 / 21 & 11: 51: 17.579 & 33.176 & -115.608 & 0.4 \\ 2010 / 05 / 21 & 17: 31: 58.579 & 33.176 & -115.608 & 0.4 \\ 2010 / 05 / 21 & 17: 43: 18.579 & 33.188 & -115.599 & 0.8 \\ 2010 / 05 / 21 & 21: 13: 32.579 & 33.118 & -115.610 & 9.1 \\ 2010 / 05 / 22 & 07: 57: 19.579 & 33.179 & -115.614 & 1.8 \\ 2010 / 05 / 22 & 08: 00: 59.579 & 33.181 & -115.659 & 6.0 \\ 2010 / 05 / 22 & 09: 28: 52.579 & 33.188 & -115.599 & 0.8 \\ 2010 / 05 / 22 & 09: 31: 19.579 & 33.181 & -115.665 & 4.2 \\ 2010 / 05 / 22 & 09: 39: 18.579 & 33.193 & -115.583 & 5.9 \\ 2010 / 05 / 22 & 11: 52: 16.579 & 33.213 & -115.584 & 1.3 \\ 2010 / 05 / 22 & 12: 15: 34.579 & 33.176 & -115.609 & 2.1 \\ 2010 / 05 / 22 & 12: 29: 50.579 & 33.192 & -115.572 & 1.6 \\ 2010 / 05 / 22 & 12: 49: 44.579 & 33.176 & -115.608 & 0.4 \\ 2010 / 05 / 22 & 16: 34: 42.579 & 33.241 & -115.633 & 3.4 \\ 2010 / 05 / 22 & 20: 00: 22.579 & 33.130 & -115.634 & 0.0 \\ 2010 / 05 / 29 & 20: 36: 09.579 & 33.176 & -115.609 & 2.1 \\ 2010 / 05 / 29 & 05: 38: 09.639 & 33.205 & -115.577 & 0.0 \\ 2010 / 05 / 29 & 22: 46: 21.639 & 33.115 & -115.655 & 4.8 \\ 205 / 30 & 07: 04: 22.579 & 33.231 & -115.605 & 4.8 \\ 20 & 16: 13: 05.579 & 33.140 & -115.636 & 7.3\end{array}$




$\begin{array}{lllll}2010 / 05 / 31 & 02: 31: 46.639 & 33.175 & -115.600 & 1.1 \\ 2010 / 05 / 31 & 15: 04: 43.639 & 33.135 & -115.644 & 6.9 \\ 2010 / 06 / 01 & 04: 46: 33.639 & 33.149 & -115.640 & 3.5 \\ 2010 / 06 / 01 & 23: 09: 37.639 & 33.149 & -115.640 & 3.5 \\ 2010 / 06 / 02 & 07: 24: 13.639 & 33.160 & -115.633 & 4.6 \\ 2010 / 06 / 03 & 00: 45: 34.639 & 33.108 & -115.602 & 8.1 \\ 2010 / 06 / 03 & 01: 20: 49.639 & 33.154 & -115.672 & 0.3 \\ 2010 / 06 / 03 & 04: 56: 52.639 & 33.196 & -115.587 & 4.5 \\ 2010 / 06 / 03 & 05: 35: 28.639 & 33.165 & -115.643 & 1.7 \\ 2010 / 06 / 03 & 09: 52: 08.639 & 33.181 & -115.610 & 0.9 \\ 2010 / 06 / 03 & 12: 21: 29.639 & 33.226 & -115.588 & 4.6 \\ 2010 / 06 / 03 & 21: 51: 11.639 & 33.190 & -115.604 & 1.2 \\ 2010 / 06 / 03 & 21: 52: 17.639 & 33.179 & -115.594 & 1.5 \\ 2010 / 06 / 03 & 21: 54: 04.639 & 33.184 & -115.606 & 2.9 \\ 2010 / 06 / 03 & 21: 55: 32.639 & 33.203 & -115.578 & 2.2 \\ 2010 / 06 / 03 & 21: 57: 21.639 & 33.179 & -115.594 & 1.5 \\ 2010 / 06 / 03 & 21: 59: 39.639 & 33.179 & -115.594 & 1.5 \\ 2010 / 06 / 03 & 22: 00: 15.639 & 33.190 & -115.604 & 1.2 \\ 2010 / 06 / 03 & 22: 02: 36.639 & 33.181 & -115.665 & 4.2 \\ 2010 / 06 / 03 & 22: 03: 56.639 & 33.181 & -115.665 & 4.2 \\ 2010 / 06 / 03 & 22: 04: 31.639 & 33.181 & -115.608 & 1.0 \\ 2010 / 06 / 03 & 22: 07: 27.639 & 33.181 & -115.665 & 4.2 \\ 2010 / 06 / 03 & 22: 12: 30.639 & 33.181 & -115.665 & 4.2 \\ 2010 / 06 / 03 & 22: 13: 10.639 & 33.179 & -115.594 & 1.5 \\ 2010 / 06 / 03 & 22: 26: 45.639 & 33.181 & -115.665 & 4.2 \\ 2010 / 06 / 03 & 22: 31: 11.639 & 33.184 & -115.608 & 0.3 \\ 2010 / 06 / 03 & 22: 36: 57.639 & 33.179 & -115.614 & 1.8 \\ 2010 / 06 / 04 & 01: 28: 30.639 & 33.251 & -115.564 & 0.1 \\ 2010 / 06 / 04 & 02: 21: 10.639 & 33.212 & -115.610 & 4.1 \\ 2010 / 06 / 04 & 05: 49: 14.639 & 33.193 & -115.583 & 5.9 \\ 2010 / 06 / 04 & 12: 53: 35.639 & 33.170 & -115.600 & 1.1 \\ 2010 / 06 / 05 & 02: 27: 09.639 & 33.194 & -115.567 & 3.1 \\ 2010 / 06 / 06 & 03: 36: 20.639 & 33.196 & -115.559 & 0.2 \\ 2010 / 06 / 07 & 14: 33: 05.639 & 33.176 & -115.608 & 0.4 \\ 2010 / 06 / 08 & 08: 56: 30.479 & 33.186 & -115.624 & 2.5 \\ 2010 / 06 / 08 & 09: 12: 46.479 & 33.179 & -115.594 & 1.5 \\ 2010 / 06 / 08 & 10: 41: 51.479 & 33.176 & -115.609 & 2.1 \\ 2010 / 06 / 08 & 11: 29: 32.479 & 33.196 & -115.587 & 5.3 \\ 2010 / 06 / 08 & 11: 29: 46.479 & 33.196 & -115.587 & 5.3 \\ 2010 / 06 / 08 & 11: 34: 46.479 & 33.196 & -115.587 & 5.3 \\ 2010 / 06 / 08 & 11: 36: 53.479 & 33.181 & -115.665 & 4.2 \\ 2010 / 06 / 08 & 11: 40: 27.479 & 33.188 & -115.573 & 0.2 \\ 2010 / 06 / 08 & 13: 39: 31.479 & 33.161 & -115.644 & 1.0 \\ 2: 12: 47.479 & 33.176 & -115.631 & 5.0 \\ 209 & 01: 20: 58.479 & 33.117 & -115.613 & 7.9\end{array}$




$\begin{array}{lllll}2010 / 06 / 09 & 05: 02: 35.479 & 33.182 & -115.602 & 2.5 \\ 2010 / 06 / 09 & 05: 43: 50.479 & 33.201 & -115.584 & 3.7 \\ 2010 / 06 / 09 & 06: 40: 11.479 & 33.188 & -115.599 & 0.8 \\ 2010 / 06 / 09 & 23: 03: 51.479 & 33.208 & -115.631 & 5.0 \\ 2010 / 06 / 09 & 23: 05: 26.479 & 33.160 & -115.633 & 4.6 \\ 2010 / 06 / 10 & 06: 43: 04.479 & 33.231 & -115.657 & 2.0 \\ 2010 / 06 / 10 & 15: 25: 39.479 & 33.208 & -115.631 & 5.0 \\ 2010 / 06 / 11 & 00: 14: 24.479 & 33.191 & -115.684 & 3.3 \\ 2010 / 06 / 11 & 00: 29: 06.479 & 33.170 & -115.626 & 0.6 \\ 2010 / 06 / 11 & 01: 49: 27.479 & 33.182 & -115.603 & 0.1 \\ 2010 / 06 / 11 & 19: 53: 02.479 & 33.231 & -115.655 & 4.8 \\ 2010 / 06 / 12 & 05: 06: 14.479 & 33.157 & -115.641 & 2.2 \\ 2010 / 06 / 12 & 05: 06: 33.479 & 33.157 & -115.641 & 2.2 \\ 2010 / 06 / 12 & 05: 08: 40.479 & 33.157 & -115.641 & 2.2 \\ 2010 / 06 / 12 & 05: 40: 06.479 & 33.231 & -115.657 & 2.0 \\ 2010 / 06 / 12 & 06: 47: 10.479 & 33.175 & -115.606 & 4.2 \\ 2010 / 06 / 12 & 06: 47: 39.479 & 33.157 & -115.641 & 2.2 \\ 2010 / 06 / 12 & 06: 58: 08.479 & 33.176 & -115.609 & 3.8 \\ 2010 / 06 / 12 & 06: 59: 49.479 & 33.157 & -115.641 & 2.2 \\ 2010 / 06 / 12 & 07: 02: 51.479 & 33.157 & -115.641 & 2.2 \\ 2010 / 06 / 12 & 12: 18: 02.479 & 33.231 & -115.657 & 2.0 \\ 2010 / 06 / 12 & 17: 58: 03.479 & 33.190 & -115.612 & 1.0 \\ 2010 / 06 / 12 & 18: 04: 51.479 & 33.190 & -115.612 & 1.0 \\ 2010 / 06 / 12 & 18: 05: 42.479 & 33.190 & -115.612 & 1.0 \\ 2010 / 06 / 13 & 02: 07: 21.479 & 33.159 & -115.641 & 0.9 \\ 2010 / 06 / 13 & 03: 24: 20.479 & 33.158 & -115.638 & 0.4 \\ 2010 / 06 / 13 & 05: 15: 19.479 & 33.181 & -115.603 & 1.4 \\ 2010 / 06 / 13 & 08: 52: 11.479 & 33.108 & -115.602 & 8.1 \\ 2010 / 06 / 13 & 09: 58: 02.479 & 33.118 & -115.610 & 9.1 \\ 2010 / 06 / 14 & 08: 00: 16.479 & 33.184 & -115.608 & 0.3 \\ 2010 / 06 / 14 & 08: 05: 38.479 & 33.179 & -115.614 & 1.8 \\ 2010 / 06 / 14 & 08: 09: 20.479 & 33.184 & -115.608 & 0.3 \\ 2010 / 06 / 14 & 08: 49: 10.479 & 33.195 & -115.572 & 0.1 \\ 2010 / 06 / 14 & 08: 56: 34.479 & 33.195 & -115.572 & 0.1 \\ 2010 / 06 / 14 & 21: 11: 52.479 & 33.108 & -115.602 & 8.1 \\ 2010 / 06 / 14 & 21: 37: 42.479 & 33.159 & -115.641 & 0.9 \\ 2010 / 06 / 14 & 21: 55: 56.479 & 33.176 & -115.609 & 2.1 \\ 2010 / 06 / 14 & 22: 38: 44.479 & 33.181 & -115.608 & 1.0 \\ 2010 / 06 / 15 & 16: 45: 25.479 & 33.140 & -115.636 & 7.3 \\ 2010 / 06 / 17 & 07: 06: 12.639 & 33.181 & -115.608 & 1.0 \\ 2010 / 06 / 18 & 00: 12: 45.639 & 33.183 & -115.599 & 0.1 \\ 2010 / 06 / 18 & 02: 26: 43.639 & 33.208 & -115.631 & 5.0 \\ 2010 / 06 / 18 & 17: 19: 53.639 & 33.183 & -115.617 & 4.3 \\ 2010 / 06 / 19 & 00: 16: 31.639 & 33.154 & -115.672 & 0.3 \\ 2010 / 06 / 19 & 01: 27: 31.639 & 33.208 & -115.582 & 2.5 \\ & 11: 16: 15.639 & 33.165 & -115.623 & 7.0\end{array}$




$\begin{array}{lllll}2010 / 06 / 20 & 20: 11: 37.639 & 33.108 & -115.602 & 8.1 \\ 2010 / 06 / 20 & 20: 15: 29.639 & 33.149 & -115.640 & 3.5 \\ 2010 / 06 / 21 & 08: 57: 08.639 & 33.181 & -115.659 & 6.0 \\ 2010 / 06 / 21 & 22: 11: 22.639 & 33.193 & -115.602 & 0.0 \\ 2010 / 06 / 22 & 00: 51: 03.639 & 33.181 & -115.612 & 1.1 \\ 2010 / 06 / 22 & 01: 06: 51.639 & 33.173 & -115.609 & 0.1 \\ 2010 / 06 / 22 & 03: 11: 29.639 & 33.157 & -115.668 & 0.1 \\ 2010 / 06 / 22 & 03: 17: 42.639 & 33.157 & -115.668 & 0.1 \\ 2010 / 06 / 22 & 19: 43: 26.639 & 33.229 & -115.576 & 3.4 \\ 2010 / 06 / 23 & 01: 30: 52.639 & 33.183 & -115.617 & 4.3 \\ 2010 / 06 / 23 & 01: 51: 15.639 & 33.190 & -115.612 & 1.0 \\ 2010 / 06 / 23 & 10: 36: 31.639 & 33.213 & -115.584 & 1.3 \\ 2010 / 06 / 23 & 12: 39: 28.639 & 33.181 & -115.608 & 0.0 \\ 2010 / 06 / 23 & 14: 50: 38.639 & 33.100 & -115.590 & 14.6 \\ 2010 / 06 / 23 & 23: 34: 32.639 & 33.142 & -115.628 & 10.3 \\ 2010 / 06 / 24 & 02: 23: 06.639 & 33.198 & -115.593 & 2.3 \\ 2010 / 06 / 25 & 07: 24: 31.639 & 33.179 & -115.594 & 1.5 \\ 2010 / 06 / 25 & 07: 27: 08.639 & 33.179 & -115.594 & 1.5 \\ 2010 / 06 / 25 & 07: 27: 45.639 & 33.179 & -115.594 & 1.5 \\ 2010 / 06 / 25 & 07: 57: 44.639 & 33.179 & -115.594 & 1.5 \\ 2010 / 06 / 25 & 18: 12: 34.639 & 33.142 & -115.628 & 10.3 \\ 2010 / 06 / 26 & 00: 26: 46.639 & 33.179 & -115.602 & 0.6 \\ 2010 / 06 / 26 & 01: 17: 35.639 & 33.145 & -115.634 & 4.8 \\ 2010 / 06 / 26 & 09: 39: 27.639 & 33.157 & -115.668 & 0.1 \\ 2010 / 06 / 26 & 11: 18: 17.639 & 33.181 & -115.608 & 1.0 \\ 2010 / 06 / 27 & 18: 00: 46.639 & 33.171 & -115.605 & 0.7 \\ 2010 / 06 / 27 & 18: 01: 21.639 & 33.173 & -115.609 & 0.1 \\ 2010 / 06 / 28 & 00: 28: 23.639 & 33.214 & -115.620 & 5.3 \\ 2010 / 06 / 28 & 18: 43: 20.639 & 33.147 & -115.623 & 7.9 \\ 2010 / 06 / 28 & 23: 31: 21.639 & 33.207 & -115.580 & 1.9 \\ 2010 / 06 / 29 & 03: 56: 54.639 & 33.179 & -115.614 & 1.8 \\ 2010 / 06 / 29 & 09: 50: 00.639 & 33.248 & -115.633 & 2.9 \\ 2010 / 06 / 29 & 09: 53: 33.639 & 33.248 & -115.633 & 2.9 \\ 2010 / 06 / 29 & 11: 41: 37.639 & 33.140 & -115.636 & 7.3 \\ 2010 / 06 / 29 & 11: 41: 56.639 & 33.142 & -115.628 & 10.3 \\ 2010 / 06 / 29 & 13: 39: 55.639 & 33.140 & -115.636 & 7.3 \\ 2010 / 06 / 29 & 18: 21: 42.639 & 33.177 & -115.635 & 5.3 \\ 2010 / 06 / 30 & 19: 24: 25.459 & 33.185 & -115.569 & 3.7 \\ 2010 / 07 / 01 & 04: 37: 59.639 & 33.142 & -115.628 & 10.3 \\ 2010 / 07 / 01 & 16: 56: 45.639 & 33.185 & -115.569 & 3.7 \\ 2010 / 07 / 01 & 16: 58: 02.639 & 33.179 & -115.614 & 1.8 \\ 2010 / 07 / 01 & 19: 20: 47.639 & 33.179 & -115.614 & 1.8 \\ 2010 / 07 / 02 & 09: 14: 40.639 & 33.149 & -115.640 & 3.5 \\ 2010 / 07 / 02 & 15: 46: 34.639 & 33.197 & -115.614 & 5.3 \\ 2010 / 07 / 03 & 03: 26: 08.639 & 33.157 & -115.648 & 0.1 \\ 2010 / 07 / 04 & 00: 42: 37.639 & 33.229 & -115.576 & 3.4\end{array}$




$\begin{array}{lllll}2010 / 07 / 04 & 00: 52: 51.639 & 33.218 & -115.639 & 3.0 \\ 2010 / 07 / 04 & 11: 08: 40.639 & 33.170 & -115.600 & 1.1 \\ 2010 / 07 / 04 & 14: 16: 37.639 & 33.159 & -115.640 & 1.5 \\ 2010 / 07 / 04 & 14: 19: 18.639 & 33.159 & -115.640 & 1.5 \\ 2010 / 07 / 04 & 14: 25: 19.639 & 33.159 & -115.640 & 1.5 \\ 2010 / 07 / 04 & 14: 26: 46.639 & 33.159 & -115.640 & 1.5 \\ 2010 / 07 / 04 & 18: 56: 14.639 & 33.130 & -115.634 & 0.0 \\ 2010 / 07 / 04 & 23: 52: 51.639 & 33.186 & -115.612 & 0.6 \\ 2010 / 07 / 05 & 22: 34: 30.639 & 33.192 & -115.572 & 1.6 \\ 2010 / 07 / 06 & 14: 50: 54.639 & 33.181 & -115.629 & 4.0 \\ 2010 / 07 / 06 & 15: 55: 10.639 & 33.135 & -115.644 & 6.9 \\ 2010 / 07 / 07 & 13: 33: 40.639 & 33.218 & -115.639 & 3.0 \\ 2010 / 07 / 07 & 13: 34: 22.639 & 33.218 & -115.639 & 3.0 \\ 2010 / 07 / 07 & 17: 37: 26.639 & 33.117 & -115.613 & 7.9 \\ 2010 / 07 / 08 & 07: 38: 11.639 & 33.176 & -115.604 & 0.0 \\ 2010 / 07 / 08 & 14: 05: 55.639 & 33.183 & -115.599 & 0.1 \\ 2010 / 07 / 08 & 14: 29: 34.639 & 33.226 & -115.588 & 4.6 \\ 2010 / 07 / 09 & 10: 09: 12.639 & 33.192 & -115.567 & 3.1 \\ 2010 / 07 / 09 & 10: 10: 51.639 & 33.194 & -115.567 & 3.1 \\ 2010 / 07 / 09 & 17: 13: 03.639 & 33.118 & -115.590 & 13.8 \\ 2010 / 07 / 09 & 18: 38: 57.639 & 33.108 & -115.602 & 8.1 \\ 2010 / 07 / 09 & 23: 31: 29.639 & 33.117 & -115.613 & 7.9 \\ 2010 / 07 / 10 & 03: 10: 46.641 & 33.193 & -115.615 & 3.9 \\ 2010 / 07 / 10 & 03: 15: 35.641 & 33.180 & -115.609 & 0.0 \\ 2010 / 07 / 10 & 05: 13: 22.641 & 33.183 & -115.599 & 0.1 \\ 2010 / 07 / 10 & 05: 47: 19.641 & 33.186 & -115.612 & 0.6 \\ 2010 / 07 / 10 & 05: 57: 26.641 & 33.179 & -115.594 & 1.5 \\ 2010 / 07 / 10 & 09: 52: 26.641 & 33.130 & -115.634 & 0.0 \\ 2010 / 07 / 10 & 11: 29: 41.641 & 33.154 & -115.672 & 0.3 \\ 2010 / 07 / 10 & 11: 30: 38.641 & 33.154 & -115.672 & 0.3 \\ 2010 / 07 / 10 & 11: 32: 47.641 & 33.164 & -115.668 & 0.0 \\ 2010 / 07 / 11 & 04: 15: 12.641 & 33.181 & -115.608 & 0.0 \\ 2010 / 07 / 11 & 04: 58: 29.641 & 33.181 & -115.665 & 4.2 \\ 2010 / 07 / 11 & 10: 50: 44.641 & 33.182 & -115.603 & 0.1 \\ 2010 / 07 / 11 & 18: 13: 02.641 & 33.190 & -115.608 & 2.2 \\ 2010 / 07 / 12 & 09: 51: 24.641 & 33.177 & -115.606 & 0.1 \\ 2010 / 07 / 12 & 13: 03: 17.641 & 33.177 & -115.606 & 0.1 \\ 2010 / 07 / 13 & 07: 23: 52.641 & 33.117 & -115.613 & 7.9 \\ 2010 / 07 / 13 & 15: 13: 57.641 & 33.118 & -115.610 & 9.1 \\ 2010 / 07 / 14 & 17: 13: 27.641 & 33.207 & -115.580 & 1.9 \\ 2010 / 07 / 14 & 17: 13: 54.641 & 33.207 & -115.580 & 1.9 \\ 2010 / 07 / 15 & 11: 15: 18.641 & 33.161 & -115.651 & 0.7 \\ 2010 / 07 / 16 & 03: 36: 07.641 & 33.192 & -115.579 & 4.3 \\ 2010 / 07 / 16 & 04: 02: 35.641 & 33.201 & -115.544 & 3.4 \\ 207 / 16 & 07: 56: 26.641 & 33.181 & -115.665 & 4.2 \\ 205: 23: 56.641 & 33.158 & -115.638 & 0.4\end{array}$




$\begin{array}{lllll}2010 / 07 / 18 & 03: 43: 27.641 & 33.193 & -115.615 & 3.9 \\ 2010 / 07 / 18 & 05: 24: 17.641 & 33.115 & -115.585 & 6.5 \\ 2010 / 07 / 18 & 05: 53: 23.641 & 33.115 & -115.585 & 6.5 \\ 2010 / 07 / 18 & 14: 00: 35.641 & 33.108 & -115.602 & 8.1 \\ 2010 / 07 / 18 & 20: 30: 16.641 & 33.142 & -115.628 & 10.3 \\ 2010 / 07 / 19 & 20: 44: 34.641 & 33.176 & -115.609 & 2.1 \\ 2010 / 07 / 20 & 03: 18: 15.641 & 33.157 & -115.680 & 0.2 \\ 2010 / 07 / 20 & 03: 19: 15.641 & 33.100 & -115.590 & 14.6 \\ 2010 / 07 / 20 & 09: 23: 43.641 & 33.208 & -115.582 & 2.5 \\ 2010 / 07 / 20 & 15: 23: 36.641 & 33.201 & -115.544 & 3.4 \\ 2010 / 07 / 21 & 00: 38: 43.641 & 33.175 & -115.606 & 4.2 \\ 2010 / 07 / 21 & 05: 33: 54.641 & 33.157 & -115.680 & 0.2 \\ 2010 / 07 / 21 & 06: 54: 10.641 & 33.157 & -115.680 & 0.2 \\ 2010 / 07 / 22 & 23: 06: 42.641 & 33.203 & -115.578 & 2.2 \\ 2010 / 07 / 24 & 17: 29: 42.641 & 33.115 & -115.585 & 6.5 \\ 2010 / 07 / 24 & 19: 10: 03.641 & 33.159 & -115.650 & 1.5 \\ 2010 / 07 / 24 & 22: 41: 47.641 & 33.139 & -115.602 & 7.0 \\ 2010 / 07 / 25 & 00: 20: 05.641 & 33.188 & -115.599 & 0.8 \\ 2010 / 07 / 25 & 18: 26: 38.641 & 33.182 & -115.606 & 1.7 \\ 2010 / 07 / 25 & 23: 29: 31.641 & 33.157 & -115.668 & 0.1 \\ 2010 / 07 / 25 & 23: 33: 30.641 & 33.158 & -115.642 & 0.1 \\ 2010 / 07 / 26 & 02: 07: 01.641 & 33.202 & -115.671 & 6.3 \\ 2010 / 07 / 26 & 03: 55: 30.641 & 33.108 & -115.602 & 8.1 \\ 2010 / 07 / 26 & 04: 39: 55.641 & 33.108 & -115.602 & 8.1 \\ 2010 / 07 / 26 & 18: 14: 45.641 & 33.139 & -115.602 & 7.0 \\ 2010 / 07 / 26 & 21: 02: 00.641 & 33.181 & -115.608 & 0.0 \\ 2010 / 07 / 26 & 21: 12: 55.641 & 33.108 & -115.602 & 8.1 \\ 2010 / 07 / 27 & 00: 22: 29.641 & 33.164 & -115.668 & 0.0 \\ 2010 / 07 / 27 & 06: 22: 30.641 & 33.139 & -115.602 & 7.0 \\ 2010 / 07 / 27 & 09: 55: 08.641 & 33.176 & -115.609 & 3.8 \\ 2010 / 07 / 27 & 11: 50: 51.641 & 33.108 & -115.602 & 8.1 \\ 2010 / 07 / 27 & 12: 00: 53.641 & 33.205 & -115.577 & 0.0 \\ 2010 / 07 / 27 & 12: 01: 05.641 & 33.205 & -115.577 & 0.0 \\ 2010 / 07 / 27 & 16: 14: 22.641 & 33.160 & -115.633 & 4.6 \\ 2010 / 07 / 27 & 16: 15: 40.641 & 33.160 & -115.633 & 4.6 \\ 2010 / 07 / 27 & 16: 16: 25.641 & 33.159 & -115.635 & 7.3 \\ 2010 / 07 / 27 & 16: 23: 00.641 & 33.196 & -115.587 & 5.3 \\ 2010 / 07 / 27 & 20: 07: 39.641 & 33.193 & -115.583 & 5.9 \\ 2010 / 07 / 28 & 05: 51: 48.641 & 33.176 & -115.608 & 0.4 \\ 2010 / 07 / 28 & 15: 11: 16.641 & 33.229 & -115.576 & 3.4 \\ 2010 / 07 / 29 & 04: 02: 47.641 & 33.241 & -115.633 & 3.4 \\ 2010 / 07 / 29 & 14: 45: 11.641 & 33.117 & -115.613 & 7.9 \\ 2010 / 07 / 29 & 15: 06: 59.641 & 33.190 & -115.604 & 1.2 \\ 2010 / 07 / 30 & 08: 08: 37.641 & 33.177 & -115.621 & 2.7 \\ 2010 / 07 / 30 & 08: 09: 27.641 & 33.176 & -115.609 & 2.1 \\ 2010 / 07 / 30 & 08: 10: 47.641 & 33.177 & -115.606 & 0.1\end{array}$




$\begin{array}{lllll}2010 / 07 / 30 & 09: 00: 29.641 & 33.181 & -115.608 & 0.0 \\ 2010 / 07 / 31 & 07: 25: 15.641 & 33.184 & -115.604 & 0.1 \\ 2010 / 07 / 31 & 12: 46: 28.641 & 33.181 & -115.610 & 0.9 \\ 2010 / 07 / 31 & 18: 02: 43.641 & 33.141 & -115.641 & 4.5 \\ 2010 / 08 / 01 & 01: 12: 04.641 & 33.205 & -115.577 & 0.0 \\ 2010 / 08 / 01 & 02: 13: 57.641 & 33.180 & -115.609 & 0.0 \\ 2010 / 08 / 01 & 05: 12: 01.641 & 33.205 & -115.577 & 0.0 \\ 2010 / 08 / 01 & 12: 32: 44.641 & 33.135 & -115.644 & 6.9 \\ 2010 / 08 / 02 & 05: 40: 37.641 & 33.170 & -115.626 & 0.6 \\ 2010 / 08 / 02 & 05: 47: 43.641 & 33.117 & -115.613 & 7.9 \\ 2010 / 08 / 02 & 05: 48: 03.641 & 33.188 & -115.573 & 0.2 \\ 2010 / 08 / 02 & 05: 48: 30.641 & 33.158 & -115.642 & 0.1 \\ 2010 / 08 / 02 & 05: 49: 47.641 & 33.108 & -115.602 & 8.1 \\ 2010 / 08 / 03 & 14: 19: 54.641 & 33.218 & -115.639 & 3.0 \\ 2010 / 08 / 03 & 14: 54: 31.641 & 33.208 & -115.631 & 5.0 \\ 2010 / 08 / 03 & 16: 29: 01.641 & 33.184 & -115.609 & 2.2 \\ 2010 / 08 / 03 & 16: 31: 47.641 & 33.179 & -115.594 & 1.5 \\ 2010 / 08 / 03 & 16: 58: 20.641 & 33.184 & -115.609 & 2.2 \\ 2010 / 08 / 03 & 18: 47: 02.641 & 33.183 & -115.617 & 4.3 \\ 2010 / 08 / 03 & 19: 28: 14.641 & 33.183 & -115.617 & 4.3 \\ 2010 / 08 / 03 & 19: 35: 40.641 & 33.183 & -115.617 & 4.3 \\ 2010 / 08 / 04 & 12: 54: 39.641 & 33.176 & -115.604 & 0.5 \\ 2010 / 08 / 04 & 13: 00: 43.641 & 33.177 & -115.606 & 0.1 \\ 2010 / 08 / 05 & 01: 45: 45.641 & 33.183 & -115.599 & 0.1 \\ 2010 / 08 / 05 & 01: 46: 43.641 & 33.183 & -115.599 & 0.1 \\ 2010 / 08 / 05 & 03: 24: 53.641 & 33.208 & -115.631 & 5.0 \\ 2010 / 08 / 05 & 12: 33: 56.641 & 33.201 & -115.544 & 3.4 \\ 2010 / 08 / 05 & 16: 17: 34.641 & 33.183 & -115.677 & 0.5 \\ 2010 / 08 / 05 & 22: 07: 03.641 & 33.176 & -115.609 & 2.1 \\ 2010 / 08 / 05 & 22: 07: 14.641 & 33.176 & -115.609 & 2.1 \\ 2010 / 08 / 05 & 22: 07: 50.641 & 33.176 & -115.609 & 2.1 \\ 2010 / 08 / 05 & 22: 58: 40.641 & 33.181 & -115.639 & 3.7 \\ 2010 / 08 / 05 & 23: 04: 00.641 & 33.181 & -115.639 & 3.7 \\ 2010 / 08 / 05 & 23: 10: 24.641 & 33.181 & -115.639 & 3.7 \\ 2010 / 08 / 05 & 23: 24: 25.641 & 33.181 & -115.639 & 3.7 \\ 2010 / 08 / 05 & 23: 27: 50.641 & 33.181 & -115.639 & 3.7 \\ 2010 / 08 / 05 & 23: 30: 56.641 & 33.181 & -115.639 & 3.7 \\ 2010 / 08 / 05 & 23: 33: 21.641 & 33.181 & -115.639 & 3.7 \\ 2010 / 08 / 05 & 23: 43: 58.641 & 33.181 & -115.639 & 3.7 \\ 2010 / 08 / 05 & 23: 45: 05.641 & 33.181 & -115.639 & 3.7 \\ 2010 / 08 / 05 & 23: 45: 57.641 & 33.181 & -115.639 & 3.7 \\ 2010 / 08 / 05 & 23: 48: 02.641 & 33.181 & -115.639 & 3.7 \\ 2010 / 08 / 05 & 23: 48: 34.641 & 33.181 & -115.639 & 3.7 \\ 2010 / 08 / 05 & 23: 48: 56.641 & 33.181 & -115.639 & 3.7 \\ 2010 / 08 / 05 & 23: 52: 21.641 & 33.181 & -115.639 & 3.7\end{array}$




\begin{tabular}{lllll}
$2010 / 08 / 05$ & $23: 52: 45.641$ & 33.181 & -115.639 & 3.7 \\
$2010 / 08 / 05$ & $23: 54: 15.641$ & 33.181 & -115.639 & 3.7 \\
$2010 / 08 / 05$ & $23: 57: 34.641$ & 33.181 & -115.639 & 3.7 \\
$2010 / 08 / 06$ & $00: 03: 37.641$ & 33.181 & -115.639 & 3.7 \\
$2010 / 08 / 06$ & $00: 08: 16.641$ & 33.181 & -115.639 & 3.7 \\
$2010 / 08 / 06$ & $00: 16: 55.641$ & 33.181 & -115.639 & 3.7 \\
$2010 / 08 / 06$ & $00: 19: 06.641$ & 33.181 & -115.639 & 3.7 \\
$2010 / 08 / 06$ & $00: 19: 52.641$ & 33.181 & -115.639 & 3.7 \\
$2010 / 08 / 06$ & $00: 25: 04.641$ & 33.186 & -115.624 & 2.5 \\
$2010 / 08 / 06$ & $00: 32: 45.641$ & 33.202 & -115.671 & 6.3 \\
$2010 / 08 / 06$ & $00: 34: 35.641$ & 33.179 & -115.594 & 1.5 \\
$2010 / 08 / 06$ & $00: 40: 36.641$ & 33.181 & -115.639 & 3.7 \\
$2010 / 08 / 06$ & $00: 50: 47.641$ & 33.193 & -115.632 & 3.5 \\
$2010 / 08 / 06$ & $01: 09: 05.641$ & 33.181 & -115.639 & 3.7 \\
$2010 / 08 / 06$ & $01: 14: 07.641$ & 33.181 & -115.639 & 3.7 \\
$2010 / 08 / 06$ & $01: 21: 31.641$ & 33.188 & -115.599 & 0.8 \\
$2010 / 08 / 06$ & $01: 37: 49.641$ & 33.181 & -115.639 & 3.7 \\
$2010 / 08 / 06$ & $01: 46: 23.641$ & 33.181 & -115.639 & 3.7 \\
$2010 / 08 / 06$ & $01: 52: 45.641$ & 33.181 & -115.639 & 3.7 \\
$2010 / 08 / 06$ & $01: 54: 10.641$ & 33.181 & -115.639 & 3.7 \\
$2010 / 08 / 06$ & $02: 05: 58.641$ & 33.181 & -115.639 & 3.7 \\
$2010 / 08 / 06$ & $02: 12: 24.641$ & 33.208 & -115.582 & 2.5 \\
$2010 / 08 / 06$ & $02: 50: 07.641$ & 33.181 & -115.639 & 3.7 \\
$2010 / 08 / 06$ & $02: 55: 00.641$ & 33.181 & -115.639 & 3.7 \\
$2010 / 08 / 06$ & $03: 07: 12.641$ & 33.181 & -115.639 & 3.7 \\
$2010 / 08 / 06$ & $03: 08: 46.641$ & 33.181 & -115.639 & 3.7 \\
$2010 / 08 / 06$ & $03: 26: 57.641$ & 33.193 & -115.632 & 3.5 \\
$2010 / 08 / 06$ & $03: 32: 14.641$ & 33.241 & -115.633 & 3.4 \\
$2010 / 08 / 06$ & $05: 40: 25.641$ & 33.181 & -115.639 & 3.7 \\
$2010 / 08 / 06$ & $06: 06: 01.641$ & 33.181 & -115.639 & 3.7 \\
$2010 / 08 / 06$ & $06: 06: 21.641$ & 33.181 & -115.639 & 3.7 \\
$2010 / 08 / 06$ & $06: 09: 53.641$ & 33.181 & -115.639 & 3.7 \\
$2010 / 08 / 06$ & $06: 17: 51.641$ & 33.181 & -115.639 & 3.7 \\
$2010 / 08 / 06$ & $06: 33: 38.641$ & 33.181 & -115.639 & 3.7 \\
$2010 / 08 / 06$ & $06: 58: 20.641$ & 33.181 & -115.639 & 3.7 \\
$2010 / 08 / 06$ & $07: 11: 24.641$ & 33.181 & -115.639 & 3.7 \\
$2010 / 08 / 06$ & $08: 55: 59.641$ & 33.207 & -115.567 & 3.7 \\
$2010 / 08 / 06$ & $09: 27: 41.641$ & 33.181 & -115.639 & 3.7 \\
$2010 / 08 / 06$ & $11: 49: 58.641$ & 33.197 & -115.577 & 0.1 \\
$2010 / 08 / 06$ & $11: 52: 12.641$ & 33.185 & -115.569 & 3.7 \\
$2010 / 08 / 06$ & $13: 10: 12.641$ & 33.175 & -115.601 & 0.1 \\
$2010 / 08 / 06$ & $17: 52: 09.641$ & 33.108 & -115.602 & 8.1 \\
$2010 / 08 / 06$ & $17: 54: 21.641$ & 33.184 & -115.609 & 2.2 \\
$2010 / 08 / 08$ & $18: 13: 56.641$ & 33.157 & -115.564 & 0.1 \\
\hline & $23: 56: 13.641$ & 33.170 & -115.680 & 0.2 \\
206 & & & 0.0
\end{tabular}




$\begin{array}{lllll}2010 / 08 / 09 & 02: 47: 58.641 & 33.135 & -115.644 & 6.9 \\ 2010 / 08 / 09 & 03: 50: 51.641 & 33.158 & -115.638 & 0.4 \\ 2010 / 08 / 09 & 03: 53: 10.641 & 33.158 & -115.638 & 0.4 \\ 2010 / 08 / 09 & 04: 25: 26.641 & 33.108 & -115.602 & 8.1 \\ 2010 / 08 / 10 & 20: 06: 35.641 & 33.193 & -115.583 & 5.9 \\ 2010 / 08 / 10 & 20: 07: 26.641 & 33.203 & -115.578 & 2.2 \\ 2010 / 08 / 10 & 20: 08: 43.641 & 33.176 & -115.608 & 0.4 \\ 2010 / 08 / 10 & 20: 11: 32.641 & 33.203 & -115.578 & 2.2 \\ 2010 / 08 / 10 & 21: 41: 23.641 & 33.203 & -115.578 & 2.2 \\ 2010 / 08 / 10 & 23: 09: 24.641 & 33.203 & -115.578 & 2.2 \\ 2010 / 08 / 11 & 00: 38: 08.641 & 33.196 & -115.587 & 4.5 \\ 2010 / 08 / 11 & 00: 40: 16.641 & 33.196 & -115.573 & 0.1 \\ 2010 / 08 / 11 & 00: 41: 26.641 & 33.198 & -115.571 & 0.2 \\ 2010 / 08 / 11 & 01: 06: 58.641 & 33.115 & -115.585 & 6.5 \\ 2010 / 08 / 11 & 05: 00: 39.641 & 33.176 & -115.604 & 0.5 \\ 2010 / 08 / 11 & 05: 01: 38.641 & 33.203 & -115.578 & 2.2 \\ 2010 / 08 / 11 & 05: 10: 33.641 & 33.176 & -115.604 & 0.5 \\ 2010 / 08 / 11 & 08: 44: 38.641 & 33.192 & -115.579 & 4.3 \\ 2010 / 08 / 11 & 13: 11: 20.641 & 33.188 & -115.573 & 0.2 \\ 2010 / 08 / 11 & 13: 12: 08.641 & 33.183 & -115.599 & 0.1 \\ 2010 / 08 / 11 & 13: 12: 55.641 & 33.183 & -115.599 & 0.1 \\ 2010 / 08 / 11 & 13: 14: 54.641 & 33.180 & -115.609 & 0.0 \\ 2010 / 08 / 11 & 13: 19: 28.641 & 33.183 & -115.599 & 0.1 \\ 2010 / 08 / 11 & 23: 39: 00.641 & 33.194 & -115.583 & 3.0 \\ 2010 / 08 / 12 & 02: 50: 15.641 & 33.176 & -115.606 & 6.3 \\ 2010 / 08 / 12 & 02: 52: 46.641 & 33.176 & -115.606 & 6.3 \\ 2010 / 08 / 12 & 15: 47: 49.641 & 33.181 & -115.612 & 1.1 \\ 2010 / 08 / 12 & 15: 48: 52.641 & 33.186 & -115.612 & 0.6 \\ 2010 / 08 / 12 & 15: 54: 37.641 & 33.186 & -115.612 & 0.6 \\ 2010 / 08 / 12 & 17: 16: 41.641 & 33.193 & -115.615 & 3.9 \\ 2010 / 08 / 13 & 06: 31: 06.641 & 33.188 & -115.573 & 0.2 \\ 2010 / 08 / 13 & 07: 03: 38.641 & 33.188 & -115.573 & 0.2 \\ 2010 / 08 / 13 & 13: 48: 21.641 & 33.176 & -115.606 & 6.3 \\ 2010 / 08 / 13 & 23: 04: 08.641 & 33.176 & -115.606 & 6.3 \\ 2010 / 08 / 13 & 23: 05: 33.641 & 33.176 & -115.606 & 6.3 \\ 2010 / 08 / 13 & 23: 06: 17.641 & 33.176 & -115.606 & 6.3 \\ 2010 / 08 / 13 & 23: 06: 36.641 & 33.194 & -115.583 & 3.0 \\ 2010 / 08 / 13 & 23: 07: 03.641 & 33.176 & -115.606 & 6.3 \\ 2010 / 08 / 13 & 23: 11: 04.641 & 33.176 & -115.606 & 6.3 \\ 2010 / 08 / 13 & 23: 13: 27.641 & 33.203 & -115.578 & 2.2 \\ 2010 / 08 / 13 & 23: 15: 35.641 & 33.194 & -115.583 & 3.0 \\ 2010 / 08 / 13 & 23: 17: 18.641 & 33.194 & -115.583 & 3.0 \\ 2010 / 08 / 13 & 23: 19: 42.641 & 33.194 & -115.583 & 3.0 \\ 23: 08 / 13 & 23: 22: 05.641 & 33.196 & -115.573 & 0.1\end{array}$




$\begin{array}{lllll}2010 / 08 / 13 & 23: 29: 11.641 & 33.194 & -115.583 & 3.0 \\ 2010 / 08 / 13 & 23: 29: 44.641 & 33.194 & -115.583 & 3.0 \\ 2010 / 08 / 13 & 23: 39: 12.641 & 33.192 & -115.572 & 1.6 \\ 2010 / 08 / 13 & 23: 40: 42.641 & 33.198 & -115.571 & 0.2 \\ 2010 / 08 / 13 & 23: 48: 50.641 & 33.194 & -115.583 & 3.0 \\ 2010 / 08 / 13 & 23: 57: 35.641 & 33.196 & -115.587 & 4.5 \\ 2010 / 08 / 14 & 00: 17: 02.641 & 33.194 & -115.583 & 3.0 \\ 2010 / 08 / 14 & 00: 54: 59.641 & 33.176 & -115.604 & 0.5 \\ 2010 / 08 / 14 & 00: 55: 16.641 & 33.176 & -115.604 & 0.5 \\ 2010 / 08 / 14 & 01: 38: 12.641 & 33.182 & -115.602 & 2.5 \\ 2010 / 08 / 14 & 02: 36: 05.641 & 33.183 & -115.607 & 0.0 \\ 2010 / 08 / 14 & 02: 36: 28.641 & 33.183 & -115.607 & 0.0 \\ 2010 / 08 / 14 & 02: 41: 29.641 & 33.198 & -115.586 & 3.7 \\ 2010 / 08 / 14 & 02: 41: 53.641 & 33.198 & -115.586 & 3.7 \\ 2010 / 08 / 14 & 02: 49: 39.641 & 33.198 & -115.586 & 3.7 \\ 2010 / 08 / 14 & 03: 44: 28.641 & 33.176 & -115.606 & 6.3 \\ 2010 / 08 / 14 & 04: 17: 55.641 & 33.202 & -115.575 & 3.8 \\ 2010 / 08 / 14 & 04: 23: 52.641 & 33.198 & -115.586 & 3.7 \\ 2010 / 08 / 14 & 04: 24: 08.641 & 33.203 & -115.579 & 3.5 \\ 2010 / 08 / 14 & 04: 35: 30.641 & 33.200 & -115.590 & 3.5 \\ 2010 / 08 / 14 & 04: 39: 14.641 & 33.200 & -115.590 & 3.5 \\ 2010 / 08 / 14 & 04: 45: 32.641 & 33.200 & -115.590 & 3.5 \\ 2010 / 08 / 14 & 04: 49: 38.641 & 33.203 & -115.579 & 3.5 \\ 2010 / 08 / 14 & 05: 14: 57.641 & 33.200 & -115.590 & 3.5 \\ 2010 / 08 / 14 & 05: 49: 39.641 & 33.192 & -115.572 & 1.6 \\ 2010 / 08 / 14 & 05: 51: 31.641 & 33.192 & -115.572 & 1.6 \\ 2010 / 08 / 14 & 11: 41: 49.641 & 33.197 & -115.577 & 0.1 \\ 2010 / 08 / 14 & 11: 48: 41.641 & 33.194 & -115.567 & 3.1 \\ 2010 / 08 / 14 & 11: 50: 05.641 & 33.194 & -115.567 & 3.1 \\ 2010 / 08 / 14 & 11: 55: 45.641 & 33.192 & -115.572 & 1.6 \\ 2010 / 08 / 14 & 12: 04: 34.641 & 33.194 & -115.567 & 3.1 \\ 2010 / 08 / 14 & 12: 05: 36.641 & 33.194 & -115.567 & 3.1 \\ 2010 / 08 / 14 & 12: 43: 43.641 & 33.192 & -115.572 & 1.6 \\ 2010 / 08 / 14 & 12: 44: 06.641 & 33.192 & -115.572 & 1.6 \\ 2010 / 08 / 14 & 13: 35: 32.641 & 33.192 & -115.567 & 3.1 \\ 2010 / 08 / 14 & 13: 37: 05.641 & 33.196 & -115.587 & 4.5 \\ 2010 / 08 / 14 & 13: 41: 22.641 & 33.192 & -115.567 & 3.1 \\ 2010 / 08 / 14 & 13: 41: 55.641 & 33.192 & -115.579 & 4.3 \\ 2010 / 08 / 14 & 13: 42: 26.641 & 33.201 & -115.544 & 3.4 \\ 2010 / 08 / 14 & 13: 44: 53.641 & 33.192 & -115.572 & 1.6 \\ 2010 / 08 / 14 & 13: 49: 11.641 & 33.176 & -115.606 & 6.3 \\ 2010 / 08 / 14 & 13: 52: 09.641 & 33.194 & -115.572 & 1.6 \\ 2010 / 08 / 14 & 13: 52: 59.641 & 33.170 & -115.567 & 3.1 \\ 208 / 14 & 13: 53: 33.641 & 33.192 & -115.572 & 1.6 \\ 203: 11: 27.641 & 33.194 & -115.567 & 3.1\end{array}$




$\begin{array}{lllll}2010 / 08 / 14 & 15: 12: 10.641 & 33.192 & -115.572 & 1.6 \\ 2010 / 08 / 14 & 15: 18: 35.641 & 33.192 & -115.572 & 1.6 \\ 2010 / 08 / 14 & 15: 23: 17.641 & 33.190 & -115.574 & 3.1 \\ 2010 / 08 / 14 & 15: 24: 51.641 & 33.192 & -115.572 & 1.6 \\ 2010 / 08 / 14 & 15: 25: 28.641 & 33.192 & -115.572 & 1.6 \\ 2010 / 08 / 14 & 15: 52: 02.641 & 33.196 & -115.559 & 0.2 \\ 2010 / 08 / 14 & 16: 20: 51.641 & 33.190 & -115.574 & 3.1 \\ 2010 / 08 / 15 & 04: 27: 07.641 & 33.192 & -115.572 & 1.6 \\ 2010 / 08 / 15 & 10: 52: 31.641 & 33.226 & -115.588 & 4.6 \\ 2010 / 08 / 15 & 11: 33: 17.641 & 33.182 & -115.602 & 2.5 \\ 2010 / 08 / 15 & 11: 34: 13.641 & 33.182 & -115.602 & 2.5 \\ 2010 / 08 / 15 & 11: 55: 55.641 & 33.179 & -115.594 & 1.5 \\ 2010 / 08 / 15 & 14: 13: 09.641 & 33.184 & -115.597 & 0.1 \\ 2010 / 08 / 15 & 14: 15: 49.641 & 33.184 & -115.597 & 0.1 \\ 2010 / 08 / 15 & 14: 17: 41.641 & 33.184 & -115.597 & 0.1 \\ 2010 / 08 / 15 & 14: 40: 17.641 & 33.184 & -115.597 & 0.1 \\ 2010 / 08 / 15 & 15: 34: 30.641 & 33.130 & -115.634 & 0.0 \\ 2010 / 08 / 15 & 19: 02: 42.641 & 33.197 & -115.659 & 6.4 \\ 2010 / 08 / 16 & 02: 39: 47.641 & 33.226 & -115.588 & 4.6 \\ 2010 / 08 / 16 & 10: 42: 46.641 & 33.196 & -115.587 & 5.3 \\ 2010 / 08 / 17 & 01: 53: 48.641 & 33.197 & -115.577 & 0.1 \\ 2010 / 08 / 17 & 01: 54: 58.641 & 33.197 & -115.577 & 0.1 \\ 2010 / 08 / 17 & 09: 28: 07.641 & 33.183 & -115.617 & 4.3 \\ 2010 / 08 / 17 & 14: 43: 47.641 & 33.108 & -115.602 & 8.1 \\ 2010 / 08 / 17 & 19: 31: 20.641 & 33.228 & -115.626 & 3.7 \\ 2010 / 08 / 18 & 03: 34: 03.641 & 33.161 & -115.651 & 0.7 \\ 2010 / 08 / 18 & 08: 27: 04.641 & 33.184 & -115.609 & 2.2 \\ 2010 / 08 / 18 & 08: 28: 16.641 & 33.183 & -115.599 & 0.1 \\ 2010 / 08 / 18 & 08: 36: 22.641 & 33.183 & -115.607 & 0.0 \\ 2010 / 08 / 18 & 08: 37: 12.641 & 33.183 & -115.599 & 0.1 \\ 2010 / 08 / 18 & 08: 43: 40.641 & 33.181 & -115.610 & 0.9 \\ 2010 / 08 / 18 & 10: 18: 03.641 & 33.178 & -115.632 & 2.3 \\ 2010 / 08 / 18 & 10: 58: 10.641 & 33.182 & -115.606 & 1.7 \\ 2010 / 08 / 18 & 11: 00: 47.641 & 33.193 & -115.615 & 3.9 \\ 2010 / 08 / 18 & 11: 30: 17.641 & 33.141 & -115.641 & 4.5 \\ 2010 / 08 / 18 & 11: 30: 30.641 & 33.141 & -115.641 & 4.5 \\ 2010 / 08 / 18 & 15: 41: 00.641 & 33.193 & -115.615 & 3.9 \\ 2010 / 08 / 18 & 18: 01: 30.641 & 33.231 & -115.605 & 4.8 \\ 2010 / 08 / 18 & 18: 07: 57.641 & 33.231 & -115.605 & 4.8 \\ 2010 / 08 / 18 & 18: 10: 29.641 & 33.157 & -115.648 & 0.1 \\ 2010 / 08 / 19 & 19: 11: 50.641 & 33.135 & -115.644 & 6.9 \\ 2010 / 08 / 19 & 09: 15: 20.641 & 33.193 & -115.576 & 3.4 \\ 208 / 19 & 09: 43: 10.641 & 33.170 & -115.615 & 3.9 \\ 20: 500 & 1.1 \\ 20 & 12: 16: 58.641 & 33.193 & -115.583 & 5.9 \\ 202.641 & 33.108 & -115.602 & 8.1\end{array}$




$\begin{array}{lllll}2010 / 08 / 20 & 08: 53: 31.641 & 33.176 & -115.608 & 0.4 \\ 2010 / 08 / 20 & 09: 01: 32.641 & 33.176 & -115.604 & 0.5 \\ 2010 / 08 / 20 & 09: 06: 48.641 & 33.176 & -115.608 & 0.4 \\ 2010 / 08 / 20 & 13: 33: 37.641 & 33.183 & -115.599 & 0.1 \\ 2010 / 08 / 20 & 13: 42: 58.641 & 33.183 & -115.599 & 0.1 \\ 2010 / 08 / 21 & 08: 30: 40.641 & 33.181 & -115.608 & 1.0 \\ 2010 / 08 / 21 & 10: 47: 20.641 & 33.164 & -115.668 & 0.0 \\ 2010 / 08 / 21 & 11: 06: 16.641 & 33.157 & -115.668 & 0.1 \\ 2010 / 08 / 21 & 11: 07: 32.641 & 33.157 & -115.668 & 0.1 \\ 2010 / 08 / 21 & 11: 08: 13.641 & 33.157 & -115.668 & 0.1 \\ 2010 / 08 / 21 & 22: 45: 37.641 & 33.177 & -115.606 & 0.1 \\ 2010 / 08 / 21 & 22: 46: 46.641 & 33.177 & -115.606 & 0.1 \\ 2010 / 08 / 21 & 22: 49: 07.641 & 33.181 & -115.608 & 1.0 \\ 2010 / 08 / 21 & 22: 52: 58.641 & 33.188 & -115.609 & 2.3 \\ 2010 / 08 / 22 & 04: 37: 45.641 & 33.196 & -115.587 & 5.3 \\ 2010 / 08 / 22 & 05: 30: 36.641 & 33.181 & -115.608 & 0.0 \\ 2010 / 08 / 22 & 06: 42: 14.641 & 33.140 & -115.636 & 7.3 \\ 2010 / 08 / 22 & 06: 47: 32.641 & 33.177 & -115.606 & 0.1 \\ 2010 / 08 / 22 & 19: 06: 53.641 & 33.196 & -115.587 & 5.3 \\ 2010 / 08 / 22 & 19: 07: 36.641 & 33.196 & -115.587 & 5.3 \\ 2010 / 08 / 22 & 19: 07: 50.641 & 33.176 & -115.609 & 2.1 \\ 2010 / 08 / 22 & 19: 13: 54.641 & 33.176 & -115.604 & 0.5 \\ 2010 / 08 / 22 & 19: 32: 22.641 & 33.196 & -115.587 & 5.3 \\ 2010 / 08 / 23 & 04: 23: 15.641 & 33.185 & -115.608 & 2.0 \\ 2010 / 08 / 24 & 17: 14: 53.641 & 33.178 & -115.632 & 2.3 \\ 2010 / 08 / 24 & 23: 29: 00.641 & 33.108 & -115.602 & 8.1 \\ 2010 / 08 / 25 & 05: 47: 33.641 & 33.208 & -115.631 & 5.0 \\ 2010 / 08 / 25 & 06: 17: 01.641 & 33.118 & -115.610 & 9.1 \\ 2010 / 08 / 25 & 06: 30: 45.641 & 33.140 & -115.636 & 7.3 \\ 2010 / 08 / 25 & 08: 28: 44.641 & 33.118 & -115.610 & 9.1 \\ 2010 / 08 / 25 & 09: 23: 45.641 & 33.118 & -115.610 & 9.1 \\ 2010 / 08 / 26 & 06: 06: 44.641 & 33.181 & -115.665 & 4.2 \\ 2010 / 08 / 26 & 20: 22: 11.641 & 33.176 & -115.609 & 2.1 \\ 2010 / 08 / 26 & 20: 26: 35.641 & 33.149 & -115.640 & 3.5 \\ 2010 / 08 / 26 & 20: 28: 11.641 & 33.176 & -115.606 & 6.3 \\ 2010 / 08 / 26 & 20: 34: 53.641 & 33.181 & -115.665 & 4.2 \\ 2010 / 08 / 26 & 20: 40: 20.641 & 33.181 & -115.665 & 4.2 \\ 2010 / 08 / 26 & 20: 40: 32.641 & 33.181 & -115.665 & 4.2 \\ 2010 / 08 / 26 & 20: 43: 23.641 & 33.181 & -115.665 & 4.2 \\ 2010 / 08 / 27 & 04: 49: 19.641 & 33.175 & -115.601 & 0.1 \\ 2010 / 08 / 27 & 10: 01: 01.641 & 33.199 & -115.617 & 0.0 \\ 2010 / 08 / 27 & 10: 15: 04.641 & 33.218 & -115.639 & 3.0 \\ 2010 / 08 / 27 & 10: 21: 14.641 & 33.218 & -115.639 & 3.0 \\ 2010 / 08 / 27 & 10: 47: 34.641 & 33.218 & -115.639 & 3.0 \\ 27 / 08 / 53.641 & 33.218 & -115.639 & 3.0 \\ 27 & 11: 00: 24.641 & 33.218 & -115.639 & 3.0\end{array}$




$\begin{array}{lllll}2010 / 08 / 27 & 12: 08: 06.641 & 33.218 & -115.639 & 3.0 \\ 2010 / 08 / 27 & 12: 29: 07.641 & 33.218 & -115.639 & 3.0 \\ 2010 / 08 / 27 & 12: 36: 31.641 & 33.218 & -115.639 & 3.0 \\ 2010 / 08 / 27 & 13: 10: 22.641 & 33.218 & -115.639 & 3.0 \\ 2010 / 08 / 27 & 14: 28: 18.641 & 33.218 & -115.639 & 3.0 \\ 2010 / 08 / 27 & 14: 55: 33.641 & 33.201 & -115.544 & 3.4 \\ 2010 / 08 / 27 & 15: 58: 36.641 & 33.229 & -115.576 & 3.4 \\ 2010 / 08 / 27 & 16: 26: 21.641 & 33.218 & -115.639 & 3.0 \\ 2010 / 08 / 27 & 16: 51: 08.641 & 33.231 & -115.657 & 2.0 \\ 2010 / 08 / 27 & 16: 52: 18.641 & 33.218 & -115.639 & 3.0 \\ 2010 / 08 / 27 & 17: 32: 47.641 & 33.174 & -115.603 & 0.1 \\ 2010 / 08 / 27 & 17: 34: 01.641 & 33.218 & -115.639 & 3.0 \\ 2010 / 08 / 27 & 17: 34: 27.641 & 33.199 & -115.571 & 3.3 \\ 2010 / 08 / 27 & 17: 34: 42.641 & 33.218 & -115.639 & 3.0 \\ 2010 / 08 / 27 & 17: 36: 20.641 & 33.208 & -115.631 & 5.0 \\ 2010 / 08 / 27 & 17: 37: 02.641 & 33.218 & -115.639 & 3.0 \\ 2010 / 08 / 27 & 17: 37: 24.641 & 33.218 & -115.639 & 3.0 \\ 2010 / 08 / 27 & 17: 38: 33.641 & 33.218 & -115.639 & 3.0 \\ 2010 / 08 / 27 & 17: 39: 09.641 & 33.208 & -115.631 & 5.0 \\ 2010 / 08 / 27 & 17: 40: 03.641 & 33.208 & -115.631 & 5.0 \\ 2010 / 08 / 27 & 17: 42: 05.641 & 33.218 & -115.639 & 3.0 \\ 2010 / 08 / 27 & 17: 50: 55.641 & 33.202 & -115.575 & 3.8 \\ 2010 / 08 / 27 & 17: 53: 25.641 & 33.176 & -115.608 & 0.4 \\ 2010 / 08 / 27 & 17: 58: 55.641 & 33.203 & -115.578 & 2.2 \\ 2010 / 08 / 27 & 18: 20: 38.641 & 33.231 & -115.657 & 2.0 \\ 2010 / 08 / 27 & 19: 00: 48.641 & 33.208 & -115.631 & 5.0 \\ 2010 / 08 / 27 & 19: 18: 30.641 & 33.241 & -115.633 & 3.4 \\ 2010 / 08 / 27 & 19: 28: 33.641 & 33.231 & -115.657 & 2.0 \\ 2010 / 08 / 27 & 20: 16: 13.641 & 33.218 & -115.639 & 3.0 \\ 2010 / 08 / 27 & 22: 22: 06.641 & 33.186 & -115.593 & 0.1 \\ 2010 / 08 / 27 & 23: 11: 58.641 & 33.231 & -115.657 & 2.0 \\ 2010 / 08 / 27 & 23: 48: 31.641 & 33.218 & -115.639 & 3.0 \\ 2010 / 08 / 28 & 02: 19: 46.641 & 33.183 & -115.677 & 0.5 \\ 2010 / 08 / 28 & 03: 36: 58.641 & 33.231 & -115.605 & 4.8 \\ 2010 / 08 / 28 & 07: 58: 24.641 & 33.218 & -115.639 & 3.0 \\ 2010 / 08 / 28 & 09: 49: 17.641 & 33.231 & -115.657 & 2.0 \\ 2010 / 08 / 28 & 14: 03: 32.641 & 33.218 & -115.639 & 3.0 \\ 2010 / 08 / 28 & 14: 11: 39.641 & 33.201 & -115.544 & 3.4 \\ 2010 / 08 / 28 & 14: 13: 27.641 & 33.231 & -115.605 & 4.8 \\ 2010 / 08 / 28 & 14: 14: 41.641 & 33.199 & -115.571 & 3.3 \\ 2010 / 08 / 28 & 14: 16: 35.641 & 33.231 & -115.657 & 2.0 \\ 2010 / 08 / 28 & 14: 17: 48.641 & 33.188 & -115.571 & 3.0 \\ 2010 / 08 / 28 & 14: 19: 54.641 & 33.208 & -115.631 & 5.0 \\ 2010 / 08 / 28 & 14: 22: 47.641 & 33.218 & -115.639 & 3.0 \\ 28 & 15: 10: 07.641 & 33.218 & -115.639 & 3.0 \\ 28 & 18: 32: 46.641 & 33.183 & -115.617 & 4.3\end{array}$




$\begin{array}{lllll}2010 / 08 / 28 & 19: 49: 54.641 & 33.212 & -115.610 & 4.1 \\ 2010 / 08 / 28 & 23: 20: 21.641 & 33.156 & -115.652 & 0.2 \\ 2010 / 08 / 29 & 02: 52: 20.641 & 33.231 & -115.657 & 2.0 \\ 2010 / 08 / 29 & 03: 01: 44.641 & 33.231 & -115.657 & 2.0 \\ 2010 / 08 / 29 & 03: 02: 39.641 & 33.218 & -115.639 & 3.0 \\ 2010 / 08 / 29 & 08: 30: 43.641 & 33.231 & -115.657 & 2.0 \\ 2010 / 08 / 29 & 10: 21: 30.641 & 33.183 & -115.617 & 4.3 \\ 2010 / 08 / 29 & 10: 51: 01.641 & 33.231 & -115.657 & 2.0 \\ 2010 / 08 / 29 & 11: 19: 10.641 & 33.196 & -115.587 & 5.3 \\ 2010 / 08 / 29 & 20: 13: 40.641 & 33.218 & -115.639 & 3.0 \\ 2010 / 08 / 30 & 07: 48: 01.641 & 33.186 & -115.612 & 0.6 \\ 2010 / 08 / 30 & 10: 06: 24.641 & 33.199 & -115.617 & 0.0 \\ 2010 / 08 / 30 & 11: 17: 06.641 & 33.213 & -115.584 & 1.3 \\ 2010 / 08 / 30 & 16: 42: 55.641 & 33.201 & -115.544 & 3.4 \\ 2010 / 08 / 30 & 16: 50: 59.641 & 33.175 & -115.601 & 0.1 \\ 2010 / 08 / 30 & 21: 51: 13.641 & 33.193 & -115.583 & 5.9 \\ 2010 / 08 / 30 & 22: 49: 22.641 & 33.175 & -115.601 & 0.1 \\ 2010 / 08 / 31 & 09: 03: 07.641 & 33.117 & -115.613 & 7.9 \\ 2010 / 08 / 31 & 11: 44: 05.641 & 33.184 & -115.604 & 0.1 \\ 2010 / 08 / 31 & 12: 11: 14.641 & 33.228 & -115.626 & 3.7 \\ 2010 / 08 / 31 & 12: 12: 17.641 & 33.228 & -115.626 & 3.7 \\ 2010 / 08 / 31 & 12: 26: 30.641 & 33.228 & -115.626 & 3.7 \\ 2010 / 08 / 31 & 12: 27: 20.641 & 33.228 & -115.626 & 3.7 \\ 2010 / 08 / 31 & 12: 32: 36.641 & 33.228 & -115.626 & 3.7 \\ 2010 / 08 / 31 & 12: 33: 11.641 & 33.228 & -115.626 & 3.7 \\ 2010 / 08 / 31 & 12: 41: 17.641 & 33.228 & -115.626 & 3.7 \\ 2010 / 08 / 31 & 15: 49: 10.641 & 33.161 & -115.651 & 0.7 \\ 2010 / 08 / 31 & 17: 37: 05.641 & 33.117 & -115.613 & 7.9 \\ 2010 / 09 / 01 & 00: 37: 45.641 & 33.117 & -115.613 & 7.9 \\ 2010 / 09 / 01 & 05: 38: 29.641 & 33.180 & -115.600 & 0.1 \\ 2010 / 09 / 01 & 12: 48: 26.641 & 33.185 & -115.569 & 3.7 \\ 2010 / 09 / 01 & 12: 55: 41.641 & 33.158 & -115.638 & 0.4 \\ 2010 / 09 / 01 & 13: 05: 18.641 & 33.231 & -115.655 & 4.8 \\ 2010 / 09 / 01 & 13: 06: 43.641 & 33.139 & -115.602 & 7.0 \\ 2010 / 09 / 01 & 13: 39: 48.641 & 33.181 & -115.665 & 4.2 \\ 2010 / 09 / 01 & 13: 46: 46.641 & 33.228 & -115.626 & 3.7 \\ 2010 / 09 / 01 & 14: 54: 24.641 & 33.181 & -115.665 & 4.2 \\ 2010 / 09 / 01 & 20: 15: 06.641 & 33.193 & -115.632 & 3.5 \\ 2010 / 09 / 02 & 04: 03: 51.641 & 33.108 & -115.602 & 8.1 \\ 2010 / 09 / 02 & 04: 49: 45.641 & 33.181 & -115.608 & 1.0 \\ 2010 / 09 / 02 & 10: 01: 33.641 & 33.140 & -115.636 & 7.3 \\ 2010 / 09 / 02 & 16: 44: 49.641 & 33.208 & -115.582 & 2.5 \\ 2010 / 09 / 02 & 20: 09: 25.641 & 33.178 & -115.632 & 2.3 \\ 2010 / 09 / 03 & 16: 27: 18.641 & 33.157 & -115.648 & 0.1 \\ 2010 / 09 / 03 & 22: 49: 23.641 & 33.193 & -115.602 & 0.0\end{array}$




$\begin{array}{lllll}2010 / 09 / 03 & 23: 27: 31.641 & 33.108 & -115.602 & 8.1 \\ 2010 / 09 / 04 & 08: 26: 30.641 & 33.115 & -115.585 & 6.5 \\ 2010 / 09 / 04 & 08: 30: 48.641 & 33.178 & -115.632 & 2.3 \\ 2010 / 09 / 05 & 05: 17: 49.641 & 33.117 & -115.613 & 7.9 \\ 2010 / 09 / 05 & 11: 38: 06.641 & 33.160 & -115.633 & 4.6 \\ 2010 / 09 / 05 & 11: 48: 53.641 & 33.160 & -115.633 & 4.6 \\ 2010 / 09 / 05 & 12: 54: 09.641 & 33.158 & -115.638 & 0.4 \\ 2010 / 09 / 06 & 02: 51: 44.641 & 33.203 & -115.578 & 2.2 \\ 2010 / 09 / 06 & 04: 30: 35.641 & 33.186 & -115.593 & 0.1 \\ 2010 / 09 / 06 & 05: 19: 00.641 & 33.228 & -115.626 & 3.7 \\ 2010 / 09 / 06 & 11: 10: 31.641 & 33.154 & -115.672 & 0.3 \\ 2010 / 09 / 06 & 11: 29: 50.641 & 33.164 & -115.668 & 0.0 \\ 2010 / 09 / 06 & 11: 32: 36.641 & 33.149 & -115.640 & 3.5 \\ 2010 / 09 / 06 & 11: 40: 34.641 & 33.157 & -115.680 & 0.2 \\ 2010 / 09 / 06 & 11: 41: 48.641 & 33.158 & -115.629 & 6.0 \\ 2010 / 09 / 06 & 12: 12: 50.641 & 33.108 & -115.602 & 8.1 \\ 2010 / 09 / 07 & 03: 09: 55.641 & 33.198 & -115.571 & 0.2 \\ 2010 / 09 / 08 & 08: 15: 59.641 & 33.201 & -115.544 & 3.4 \\ 2010 / 09 / 09 & 02: 36: 54.641 & 33.130 & -115.634 & 0.0 \\ 2010 / 09 / 09 & 02: 37: 50.641 & 33.208 & -115.631 & 5.0 \\ 2010 / 09 / 09 & 03: 50: 13.641 & 33.190 & -115.608 & 2.2 \\ 2010 / 09 / 09 & 12: 25: 43.641 & 33.179 & -115.594 & 1.5 \\ 2010 / 09 / 09 & 12: 27: 34.641 & 33.179 & -115.614 & 1.8 \\ 2010 / 09 / 09 & 12: 28: 52.641 & 33.229 & -115.576 & 3.4 \\ 2010 / 09 / 09 & 12: 34: 10.641 & 33.192 & -115.626 & 7.7 \\ 2010 / 09 / 09 & 12: 39: 15.641 & 33.179 & -115.594 & 1.5 \\ 2010 / 09 / 09 & 12: 39: 50.641 & 33.212 & -115.590 & 7.2 \\ 2010 / 09 / 09 & 12: 40: 34.641 & 33.179 & -115.594 & 1.5 \\ 2010 / 09 / 09 & 12: 42: 20.641 & 33.184 & -115.608 & 0.3 \\ 2010 / 09 / 09 & 12: 52: 07.641 & 33.208 & -115.582 & 2.5 \\ 2010 / 09 / 09 & 13: 11: 08.641 & 33.184 & -115.601 & 3.5 \\ 2010 / 09 / 09 & 15: 17: 50.641 & 33.208 & -115.582 & 2.5 \\ 2010 / 09 / 09 & 15: 20: 35.641 & 33.197 & -115.577 & 0.1 \\ 2010 / 09 / 09 & 15: 52: 09.641 & 33.177 & -115.607 & 4.2 \\ 2010 / 09 / 10 & 01: 24: 05.641 & 33.157 & -115.668 & 0.1 \\ 2010 / 09 / 10 & 01: 30: 25.641 & 33.161 & -115.651 & 0.7 \\ 2010 / 09 / 10 & 01: 31: 33.641 & 33.161 & -115.651 & 0.7 \\ 2010 / 09 / 10 & 01: 34: 21.641 & 33.176 & -115.609 & 2.1 \\ 2010 / 09 / 10 & 01: 34: 49.641 & 33.181 & -115.608 & 1.0 \\ 2010 / 09 / 10 & 14: 35: 38.641 & 33.184 & -115.597 & 0.1 \\ 2010 / 09 / 10 & 15: 05: 53.641 & 33.181 & -115.665 & 4.2 \\ 2010 / 09 / 10 & 19: 03: 22.641 & 33.214 & -115.620 & 5.3 \\ 2010 / 09 / 10 & 19: 22: 10.641 & 33.183 & -115.606 & 0.1 \\ 2010 / 09 / 10 & 21: 53: 51.641 & 33.194 & -115.583 & 3.0 \\ 2010 / 09 / 11 & 09: 39: 57.641 & 33.100 & -115.590 & 14.6 \\ 2010 / 09 / 11 & 16: 56: 35.641 & 33.182 & -115.603 & 0.1\end{array}$




\begin{tabular}{lllll}
$2010 / 09 / 11$ & $20: 22: 44.641$ & 33.231 & -115.605 & 4.8 \\
$2010 / 09 / 11$ & $20: 37: 56.641$ & 33.142 & -115.628 & 10.3 \\
$2010 / 09 / 11$ & $20: 38: 23.641$ & 33.108 & -115.602 & 8.1 \\
$2010 / 09 / 12$ & $00: 15: 29.641$ & 33.159 & -115.640 & 1.5 \\
$2010 / 09 / 12$ & $00: 32: 32.641$ & 33.159 & -115.640 & 1.5 \\
$2010 / 09 / 12$ & $00: 35: 52.641$ & 33.159 & -115.640 & 1.5 \\
$2010 / 09 / 12$ & $00: 36: 09.641$ & 33.159 & -115.640 & 1.5 \\
$2010 / 09 / 12$ & $00: 38: 05.641$ & 33.159 & -115.640 & 1.5 \\
$2010 / 09 / 12$ & $00: 42: 39.641$ & 33.159 & -115.640 & 1.5 \\
$2010 / 09 / 12$ & $00: 58: 22.641$ & 33.159 & -115.640 & 1.5 \\
$2010 / 09 / 12$ & $08: 28: 16.641$ & 33.190 & -115.612 & 1.0 \\
$2010 / 09 / 13$ & $05: 17: 05.641$ & 33.158 & -115.642 & 0.1 \\
$2010 / 09 / 13$ & $05: 28: 04.641$ & 33.170 & -115.654 & 0.0 \\
$2010 / 09 / 13$ & $08: 53: 20.641$ & 33.176 & -115.608 & 0.4 \\
$2010 / 09 / 13$ & $08: 58: 58.641$ & 33.179 & -115.594 & 1.5 \\
$2010 / 09 / 13$ & $10: 38: 20.641$ & 33.176 & -115.608 & 0.4 \\
$2010 / 09 / 13$ & $13: 21: 09.641$ & 33.231 & -115.657 & 2.0 \\
$2010 / 09 / 13$ & $14: 40: 16.641$ & 33.202 & -115.575 & 3.8 \\
$2010 / 09 / 13$ & $15: 07: 23.641$ & 33.108 & -115.602 & 8.1 \\
$2010 / 09 / 14$ & $18: 04: 04.641$ & 33.148 & -115.633 & 4.3 \\
$2010 / 09 / 14$ & $18: 04: 34.641$ & 33.194 & -115.583 & 3.0 \\
$2010 / 09 / 15$ & $00: 23: 36.641$ & 33.158 & -115.642 & 0.1 \\
$2010 / 09 / 15$ & $00: 26: 46.641$ & 33.158 & -115.642 & 0.1 \\
$2010 / 09 / 15$ & $01: 08: 52.641$ & 33.108 & -115.602 & 8.1 \\
$2010 / 09 / 15$ & $06: 11: 17.641$ & 33.117 & -115.613 & 7.9 \\
$2010 / 09 / 15$ & $11: 48: 54.641$ & 33.188 & -115.599 & 0.8 \\
$2010 / 09 / 16$ & $12: 07: 21.641$ & 33.190 & -115.608 & 2.2 \\
$2010 / 09 / 17$ & $04: 10: 17.641$ & 33.208 & -115.582 & 2.5 \\
$2010 / 09 / 17$ & $04: 22: 36.641$ & 33.197 & -115.577 & 0.1 \\
$2010 / 09 / 17$ & $08: 55: 35.641$ & 33.181 & -115.608 & 1.0 \\
$2010 / 09 / 17$ & $08: 56: 19.641$ & 33.181 & -115.608 & 1.0 \\
$2010 / 09 / 17$ & $09: 43: 32.641$ & 33.176 & -115.609 & 2.1 \\
$2010 / 09 / 17$ & $15: 04: 53.641$ & 33.161 & -115.651 & 0.7 \\
$2010 / 09 / 17$ & $16: 56: 36.641$ & 33.220 & -115.571 & 5.3 \\
$2010 / 09 / 17$ & $16: 57: 52.641$ & 33.220 & -115.571 & 5.3 \\
$2010 / 09 / 17$ & $17: 21: 17.641$ & 33.251 & -115.564 & 0.1 \\
$2010 / 09 / 17$ & $21: 07: 16.641$ & 33.174 & -115.604 & 0.1 \\
$2010 / 09 / 17$ & $21: 16: 14.641$ & 33.174 & -115.604 & 0.1 \\
$2010 / 09 / 17$ & $21: 44: 16.641$ & 33.174 & -115.604 & 0.1 \\
$2010 / 09 / 17$ & $21: 51: 50.641$ & 33.177 & -115.614 & 1.2 \\
$2010 / 09 / 17$ & $22: 13: 09.641$ & 33.201 & -115.544 & 3.4 \\
$2010 / 09 / 18$ & $10: 39: 52.639$ & 33.159 & -115.640 & 1.5 \\
$2010 / 09 / 18$ & $13: 43: 18.639$ & 33.108 & -115.602 & 8.1 \\
$2010 / 09 / 19$ & $00: 27: 12.639$ & 33.197 & -115.659 & 6.4 \\
$2010 / 09 / 19$ & $02: 23: 19.639$ & 33.108 & -115.602 & 8.1 \\
\hline $26: 39.639$ & 33.183 & -115.599 & 0.1
\end{tabular}




$\begin{array}{lllll}2010 / 09 / 19 & 16: 27: 48.639 & 33.183 & -115.599 & 0.1 \\ 2010 / 09 / 19 & 20: 16: 38.639 & 33.177 & -115.606 & 0.1 \\ 2010 / 09 / 19 & 20: 24: 21.639 & 33.177 & -115.606 & 0.1 \\ 2010 / 09 / 20 & 02: 48: 22.639 & 33.202 & -115.671 & 6.3 \\ 2010 / 09 / 20 & 17: 09: 35.639 & 33.176 & -115.609 & 2.1 \\ 2010 / 09 / 20 & 21: 14: 14.639 & 33.185 & -115.569 & 3.7 \\ 2010 / 09 / 20 & 21: 15: 37.639 & 33.185 & -115.569 & 3.7 \\ 2010 / 09 / 20 & 23: 52: 02.639 & 33.117 & -115.613 & 7.9 \\ 2010 / 09 / 23 & 00: 16: 45.639 & 33.181 & -115.629 & 4.0 \\ 2010 / 09 / 23 & 01: 22: 56.639 & 33.157 & -115.680 & 0.2 \\ 2010 / 09 / 23 & 01: 24: 11.639 & 33.181 & -115.612 & 1.1 \\ 2010 / 09 / 23 & 01: 45: 59.639 & 33.158 & -115.638 & 0.4 \\ 2010 / 09 / 23 & 14: 04: 15.639 & 33.181 & -115.639 & 3.7 \\ 2010 / 09 / 23 & 14: 06: 00.639 & 33.181 & -115.665 & 4.2 \\ 2010 / 09 / 23 & 21: 35: 22.639 & 33.180 & -115.600 & 0.1 \\ 2010 / 09 / 23 & 21: 37: 21.639 & 33.183 & -115.599 & 0.1 \\ 2010 / 09 / 23 & 21: 44: 49.639 & 33.173 & -115.609 & 0.1 \\ 2010 / 09 / 23 & 21: 45: 49.639 & 33.197 & -115.614 & 5.3 \\ 2010 / 09 / 23 & 21: 54: 05.639 & 33.173 & -115.609 & 0.1 \\ 2010 / 09 / 24 & 05: 57: 44.639 & 33.108 & -115.602 & 8.1 \\ 2010 / 09 / 24 & 09: 21: 46.639 & 33.176 & -115.609 & 2.1 \\ 2010 / 09 / 24 & 14: 25: 34.639 & 33.155 & -115.663 & 2.3 \\ 2010 / 09 / 24 & 20: 31: 37.639 & 33.118 & -115.610 & 9.1 \\ 2010 / 09 / 24 & 21: 49: 40.639 & 33.176 & -115.604 & 0.5 \\ 2010 / 09 / 25 & 09: 16: 30.639 & 33.185 & -115.569 & 3.7 \\ 2010 / 09 / 25 & 09: 24: 44.639 & 33.187 & -115.601 & 1.7 \\ 2010 / 09 / 25 & 11: 08: 15.639 & 33.248 & -115.633 & 2.9 \\ 2010 / 09 / 25 & 11: 42: 31.639 & 33.183 & -115.607 & 0.0 \\ 2010 / 09 / 26 & 03: 12: 51.639 & 33.100 & -115.590 & 14.6 \\ 2010 / 09 / 26 & 21: 44: 14.639 & 33.201 & -115.544 & 3.4 \\ 2010 / 09 / 27 & 02: 03: 33.639 & 33.135 & -115.644 & 6.9 \\ 2010 / 09 / 27 & 07: 44: 13.639 & 33.176 & -115.604 & 0.5 \\ 2010 / 09 / 27 & 13: 53: 08.639 & 33.181 & -115.665 & 4.2 \\ 2010 / 09 / 29 & 00: 42: 51.781 & 33.241 & -115.633 & 3.4 \\ 2010 / 09 / 29 & 02: 09: 41.781 & 33.228 & -115.626 & 3.7 \\ 2010 / 09 / 29 & 02: 12: 27.781 & 33.228 & -115.626 & 3.7 \\ 2010 / 09 / 29 & 02: 13: 12.781 & 33.228 & -115.626 & 3.7 \\ 2010 / 09 / 29 & 06: 54: 50.781 & 33.182 & -115.602 & 2.5 \\ 2010 / 09 / 29 & 06: 56: 07.781 & 33.182 & -115.602 & 2.5 \\ 2010 / 09 / 29 & 07: 00: 06.781 & 33.182 & -115.602 & 2.5 \\ 2010 / 09 / 29 & 07: 30: 31.781 & 33.179 & -115.621 & 4.8 \\ 2010 / 09 / 29 & 17: 34: 48.781 & 33.135 & -115.644 & 6.9 \\ 2010 / 09 / 30 & 03: 07: 08.781 & 33.140 & -115.636 & 7.3 \\ 2010 / 09 / 30 & 03: 35: 47.781 & 33.140 & -115.636 & 7.3 \\ 2010 / 09 / 30 & 03: 37: 08.781 & 33.108 & -115.602 & 8.1 \\ 2010 / 09 / 30 & 03: 37: 28.781 & 33.140 & -115.636 & 7.3\end{array}$




\begin{tabular}{lllll}
$2010 / 09 / 30$ & $03: 41: 43.781$ & 33.140 & -115.636 & 7.3 \\
$2010 / 09 / 30$ & $08: 18: 06.781$ & 33.184 & -115.608 & 0.3 \\
$2010 / 10 / 01$ & $14: 58: 34.781$ & 33.108 & -115.602 & 8.1 \\
$2010 / 10 / 02$ & $06: 55: 25.781$ & 33.118 & -115.590 & 13.8 \\
$2010 / 10 / 02$ & $11: 45: 24.781$ & 33.186 & -115.612 & 0.6 \\
$2010 / 10 / 02$ & $13: 05: 27.781$ & 33.203 & -115.578 & 2.2 \\
$2010 / 10 / 03$ & $01: 28: 14.781$ & 33.172 & -115.610 & 2.2 \\
$2010 / 10 / 03$ & $01: 29: 40.781$ & 33.176 & -115.604 & 0.5 \\
$2010 / 10 / 03$ & $01: 30: 50.781$ & 33.186 & -115.612 & 0.6 \\
$2010 / 10 / 03$ & $01: 42: 52.781$ & 33.160 & -115.633 & 4.6 \\
$2010 / 10 / 03$ & $08: 39: 18.781$ & 33.108 & -115.602 & 8.1 \\
$2010 / 10 / 03$ & $12: 25: 15.781$ & 33.117 & -115.613 & 7.9 \\
$2010 / 10 / 03$ & $15: 21: 17.781$ & 33.184 & -115.611 & 2.7 \\
$2010 / 10 / 04$ & $01: 05: 02.781$ & 33.158 & -115.638 & 0.4 \\
$2010 / 10 / 04$ & $01: 06: 22.781$ & 33.139 & -115.602 & 7.0 \\
$2010 / 10 / 04$ & $02: 52: 28.781$ & 33.176 & -115.618 & 3.7 \\
$2010 / 10 / 04$ & $03: 29: 18.781$ & 33.186 & -115.593 & 0.1 \\
$2010 / 10 / 04$ & $04: 58: 18.781$ & 33.161 & -115.651 & 0.7 \\
$2010 / 10 / 04$ & $05: 48: 35.781$ & 33.231 & -115.655 & 4.8 \\
$2010 / 10 / 04$ & $06: 41: 20.781$ & 33.182 & -115.602 & 2.5 \\
$2010 / 10 / 04$ & $15: 07: 22.781$ & 33.209 & -115.590 & 3.1 \\
$2010 / 10 / 04$ & $15: 44: 00.781$ & 33.213 & -115.584 & 1.3 \\
$2010 / 10 / 04$ & $19: 00: 40.781$ & 33.202 & -115.575 & 3.8 \\
$2010 / 10 / 04$ & $19: 03: 27.781$ & 33.108 & -115.602 & 8.1 \\
$2010 / 10 / 04$ & $19: 41: 27.781$ & 33.108 & -115.602 & 8.1 \\
$2010 / 10 / 04$ & $20: 07: 17.781$ & 33.231 & -115.655 & 4.8 \\
$2010 / 10 / 04$ & $22: 45: 04.781$ & 33.157 & -115.668 & 0.1 \\
$2010 / 10 / 05$ & $05: 04: 05.781$ & 33.184 & -115.611 & 2.7 \\
$2010 / 10 / 05$ & $05: 09: 38.781$ & 33.184 & -115.601 & 3.5 \\
$2010 / 10 / 05$ & $05: 41: 06.781$ & 33.208 & -115.582 & 2.5 \\
$2010 / 10 / 05$ & $05: 41: 49.781$ & 33.208 & -115.582 & 2.5 \\
$2010 / 10 / 05$ & $05: 43: 08.781$ & 33.192 & -115.626 & 7.7 \\
$2010 / 10 / 05$ & $05: 44: 09.781$ & 33.190 & -115.604 & 1.2 \\
$2010 / 10 / 05$ & $05: 44: 57.781$ & 33.184 & -115.611 & 2.7 \\
$2010 / 10 / 05$ & $05: 46: 13.781$ & 33.178 & -115.611 & 1.6 \\
$2010 / 10 / 05$ & $05: 46: 38.781$ & 33.208 & -115.582 & 2.5 \\
$2010 / 10 / 05$ & $05: 47: 27.781$ & 33.202 & -115.575 & 3.8 \\
$2010 / 10 / 05$ & $05: 47: 42.781$ & 33.202 & -115.575 & 3.8 \\
$2010 / 10 / 05$ & $05: 48: 27.781$ & 33.202 & -115.575 & 3.8 \\
$2010 / 10 / 05$ & $05: 48: 39.781$ & 33.185 & -115.569 & 3.7 \\
$2010 / 10 / 05$ & $05: 49: 11.781$ & 33.190 & -115.604 & 1.2 \\
$2010 / 10 / 05$ & $05: 49: 43.781$ & 33.208 & -115.582 & 2.5 \\
$2010 / 10 / 05$ & $05: 50: 00.781$ & 33.202 & -115.575 & 3.8 \\
\hline $25: 50: 35.781$ & 33.181 & -115.614 & 3.1 \\
$205: 51: 12.781$ & 33.208 & -115.582 & 2.5 \\
$205: 51: 28.781$ & 33.208 & -115.582 & 2.5
\end{tabular}




\begin{tabular}{lllll}
$2010 / 10 / 05$ & $05: 52: 14.781$ & 33.190 & -115.604 & 1.2 \\
$2010 / 10 / 05$ & $05: 52: 57.781$ & 33.176 & -115.608 & 0.4 \\
$2010 / 10 / 05$ & $05: 53: 53.781$ & 33.179 & -115.594 & 1.5 \\
$2010 / 10 / 05$ & $05: 54: 30.781$ & 33.202 & -115.575 & 3.8 \\
$2010 / 10 / 05$ & $05: 55: 13.781$ & 33.192 & -115.626 & 7.7 \\
$2010 / 10 / 05$ & $05: 56: 33.781$ & 33.190 & -115.608 & 2.2 \\
$2010 / 10 / 05$ & $06: 00: 45.781$ & 33.182 & -115.602 & 2.5 \\
$2010 / 10 / 05$ & $06: 01: 12.781$ & 33.208 & -115.582 & 2.5 \\
$2010 / 10 / 05$ & $06: 02: 52.781$ & 33.193 & -115.583 & 5.9 \\
$2010 / 10 / 05$ & $06: 03: 56.781$ & 33.179 & -115.614 & 1.8 \\
$2010 / 10 / 05$ & $06: 04: 25.781$ & 33.208 & -115.582 & 2.5 \\
$2010 / 10 / 05$ & $06: 05: 28.781$ & 33.193 & -115.583 & 5.9 \\
$2010 / 10 / 05$ & $06: 07: 29.781$ & 33.183 & -115.599 & 0.1 \\
$2010 / 10 / 05$ & $06: 11: 39.781$ & 33.208 & -115.582 & 2.5 \\
$2010 / 10 / 05$ & $06: 13: 47.781$ & 33.176 & -115.608 & 0.4 \\
$2010 / 10 / 05$ & $06: 14: 53.781$ & 33.182 & -115.603 & 0.1 \\
$2010 / 10 / 05$ & $06: 15: 59.781$ & 33.179 & -115.594 & 1.5 \\
$2010 / 10 / 05$ & $06: 17: 20.781$ & 33.202 & -115.575 & 3.8 \\
$2010 / 10 / 05$ & $06: 19: 44.781$ & 33.208 & -115.582 & 2.5 \\
$2010 / 10 / 05$ & $06: 21: 46.781$ & 33.187 & -115.622 & 4.0 \\
$2010 / 10 / 05$ & $06: 23: 23.781$ & 33.179 & -115.594 & 1.5 \\
$2010 / 10 / 05$ & $06: 24: 47.781$ & 33.183 & -115.599 & 0.1 \\
$2010 / 10 / 05$ & $06: 32: 48.781$ & 33.182 & -115.603 & 0.1 \\
$2010 / 10 / 05$ & $06: 33: 07.781$ & 33.231 & -115.655 & 4.8 \\
$2010 / 10 / 05$ & $06: 39: 23.781$ & 33.179 & -115.594 & 1.5 \\
$2010 / 10 / 05$ & $06: 44: 10.781$ & 33.179 & -115.614 & 1.8 \\
$2010 / 10 / 05$ & $06: 59: 05.781$ & 33.174 & -115.603 & 0.1 \\
$2010 / 10 / 05$ & $06: 59: 17.781$ & 33.179 & -115.614 & 1.8 \\
$2010 / 10 / 05$ & $07: 00: 56.781$ & 33.186 & -115.612 & 0.6 \\
$2010 / 10 / 05$ & $07: 02: 12.781$ & 33.208 & -115.582 & 2.5 \\
$2010 / 10 / 05$ & $07: 02: 45.781$ & 33.208 & -115.582 & 2.5 \\
$2010 / 10 / 05$ & $07: 43: 33.781$ & 33.202 & -115.575 & 3.8 \\
$2010 / 10 / 05$ & $07: 43: 51.781$ & 33.202 & -115.575 & 3.8 \\
$2010 / 10 / 05$ & $07: 47: 17.781$ & 33.202 & -115.575 & 3.8 \\
$2010 / 10 / 05$ & $08: 55: 13.781$ & 33.182 & -115.603 & 0.1 \\
$2010 / 10 / 05$ & $09: 01: 03.781$ & 33.182 & -115.603 & 0.1 \\
$2010 / 10 / 05$ & $09: 03: 25.781$ & 33.190 & -115.608 & 2.2 \\
$2010 / 10 / 05$ & $09: 31: 08.781$ & 33.170 & -115.600 & 1.1 \\
$2010 / 10 / 05$ & $09: 45: 01.781$ & 33.190 & -115.604 & 1.2 \\
$2010 / 10 / 05$ & $09: 55: 49.781$ & 33.179 & -115.594 & 1.5 \\
$2010 / 10 / 05$ & $09: 56: 03.781$ & 33.176 & -115.604 & 0.5 \\
$20 / 05$ & $10: 03: 34.781$ & 33.179 & -115.599 & 0.1 \\
\hline $2: 09: 18.781$ & 33.183 & -115.594 & 1.5 \\
205 & $10: 26: 48.781$ & 33.183 & -115.599 & 0.1
\end{tabular}




\begin{tabular}{|c|c|c|c|}
\hline 2010/10/05 & $10: 28: 41.781$ & 33.190 & -115.604 \\
\hline 2010/10/05 & $11: 13: 01.781$ & 33.214 & -115.620 \\
\hline 2010/10/05 & $14: 51: 48.781$ & 33.181 & -115.665 \\
\hline 2010/10/05 & $14: 52: 49.781$ & 33.176 & -115.609 \\
\hline 2010/10/05 & $14: 55: 27.781$ & 33.181 & -115.665 \\
\hline 2010/10/05 & $15: 03: 06.781$ & 33.179 & -115.594 \\
\hline 2010/10/05 & $15: 29: 57.781$ & 33.183 & -115.599 \\
\hline 2010/10/05 & $15: 44: 17.781$ & 33.188 & -115.599 \\
\hline 2010/10/05 & $19: 21: 09.781$ & 33.179 & -115.594 \\
\hline 2010/10/06 & $01: 47: 23.781$ & 33.181 & -115.665 \\
\hline 2010/10/06 & 04:49:14.781 & 33.177 & -115.606 \\
\hline 2010/10/06 & $04: 51: 23.781$ & 33.181 & -115.659 \\
\hline 2010/10/06 & $04: 52: 19.781$ & 33.176 & -115.609 \\
\hline 2010/10/06 & $05: 12: 17.781$ & 33.181 & -115.612 \\
\hline 2010/10/06 & $05: 23: 28.781$ & 33.177 & -115.606 \\
\hline 2010/10/06 & $05: 30: 32.781$ & 33.181 & -115.612 \\
\hline 2010/10/06 & $05: 33: 44.781$ & 33.181 & -115.612 \\
\hline 2010/10/06 & $05: 43: 21.781$ & 33.181 & -115.608 \\
\hline 2010/10/06 & $05: 43: 38.781$ & 33.181 & -115.608 \\
\hline 2010/10/06 & $05: 43: 53.781$ & 33.183 & -115.599 \\
\hline 2010/10/06 & $05: 45: 14.781$ & 33.183 & -115.599 \\
\hline 2010/10/06 & $05: 45: 48.781$ & 33.183 & -115.599 \\
\hline 2010/10/06 & $06: 24: 22.781$ & 33.176 & -115.618 \\
\hline 2010/10/06 & $11: 37: 38.781$ & 33.115 & -115.585 \\
\hline 2010/10/06 & 18:38:04.781 & 33.184 & -115.611 \\
\hline 2010/10/06 & $22: 28: 03.781$ & 33.202 & -115.575 \\
\hline 2010/10/07 & 07:30:47.781 & 33.198 & -115.593 \\
\hline 2010/10/07 & $08: 34: 46.781$ & 33.192 & -115.626 \\
\hline 2010/10/08 & 04:55:06.781 & 33.173 & -115.650 \\
\hline 2010/10/08 & 13:08:33.781 & 33.161 & -115.644 \\
\hline 2010/10/08 & $13: 53: 46.781$ & 33.157 & -115.680 \\
\hline 2010/10/08 & $18: 32: 36.781$ & 33.161 & -115.644 \\
\hline 2010/10/09 & $09: 21: 35.781$ & 33.182 & -115.606 \\
\hline 2010/10/09 & 09:26:44.781 & 33.179 & -115.594 \\
\hline 2010/10/09 & $13: 17: 44.781$ & 33.190 & -115.574 \\
\hline 2010/10/09 & $13: 21: 36.781$ & 33.194 & -115.583 \\
\hline 2010/10/09 & $14: 53: 40.781$ & 33.117 & -115.613 \\
\hline 2010/10/09 & $18: 59: 57.781$ & 33.190 & -115.604 \\
\hline 2010/10/09 & $19: 01: 07.781$ & 33.176 & -115.608 \\
\hline 2010/10/10 & $03: 36: 58.781$ & 33.165 & -115.643 \\
\hline 2010/10/10 & 13:38:09.781 & 33.108 & -115.602 \\
\hline 2010/10/11 & $07: 24: 40.781$ & 33.182 & -115.602 \\
\hline 2010/10/11 & $08: 47: 20.781$ & 33.176 & -115.608 \\
\hline 2010/10/12 & $06: 18: 24.781$ & 33.179 & -115.614 \\
\hline 2010/10/12 & $06: 32: 59.781$ & 33.188 & -115.599 \\
\hline 2010/10/12 & 09:50:29.781 & 33.183 & -115.607 \\
\hline
\end{tabular}




$\begin{array}{lllll}2010 / 10 / 12 & 14: 38: 09.781 & 33.182 & -115.602 & 2.5 \\ 2010 / 10 / 12 & 15: 06: 08.781 & 33.181 & -115.665 & 4.2 \\ 2010 / 10 / 12 & 18: 56: 20.781 & 33.186 & -115.612 & 0.6 \\ 2010 / 10 / 12 & 18: 56: 41.781 & 33.176 & -115.609 & 2.1 \\ 2010 / 10 / 13 & 00: 55: 56.781 & 33.176 & -115.606 & 6.3 \\ 2010 / 10 / 13 & 00: 55: 57.781 & 33.194 & -115.583 & 3.0 \\ 2010 / 10 / 13 & 01: 31: 03.781 & 33.181 & -115.608 & 0.0 \\ 2010 / 10 / 13 & 05: 51: 22.781 & 33.147 & -115.623 & 7.9 \\ 2010 / 10 / 13 & 06: 04: 36.781 & 33.221 & -115.605 & 5.1 \\ 2010 / 10 / 13 & 09: 56: 07.781 & 33.231 & -115.655 & 4.8 \\ 2010 / 10 / 13 & 11: 08: 38.781 & 33.164 & -115.632 & 2.6 \\ 2010 / 10 / 15 & 14: 47: 20.781 & 33.117 & -115.613 & 7.9 \\ 2010 / 10 / 15 & 17: 30: 11.781 & 33.140 & -115.636 & 7.3 \\ 2010 / 10 / 15 & 17: 32: 03.781 & 33.164 & -115.668 & 0.0 \\ 2010 / 10 / 15 & 17: 54: 28.781 & 33.184 & -115.597 & 0.1 \\ 2010 / 10 / 15 & 18: 13: 22.781 & 33.165 & -115.623 & 7.0 \\ 2010 / 10 / 16 & 11: 47: 15.781 & 33.147 & -115.623 & 7.9 \\ 2010 / 10 / 16 & 13: 41: 16.781 & 33.194 & -115.583 & 3.0 \\ 2010 / 10 / 16 & 16: 40: 41.781 & 33.100 & -115.590 & 14.6 \\ 2010 / 10 / 16 & 19: 05: 07.781 & 33.181 & -115.665 & 4.2 \\ 2010 / 10 / 17 & 03: 07: 17.781 & 33.200 & -115.590 & 3.5 \\ 2010 / 10 / 17 & 03: 50: 44.781 & 33.201 & -115.584 & 3.7 \\ 2010 / 10 / 17 & 05: 44: 01.781 & 33.177 & -115.606 & 0.1 \\ 2010 / 10 / 17 & 09: 46: 06.781 & 33.188 & -115.599 & 0.8 \\ 2010 / 10 / 17 & 12: 08: 08.781 & 33.170 & -115.600 & 1.1 \\ 2010 / 10 / 17 & 13: 45: 41.781 & 33.176 & -115.608 & 0.4 \\ 2010 / 10 / 17 & 14: 10: 15.781 & 33.186 & -115.612 & 0.6 \\ 2010 / 10 / 17 & 18: 36: 48.781 & 33.176 & -115.608 & 0.4 \\ 2010 / 10 / 17 & 20: 38: 15.781 & 33.193 & -115.583 & 5.9 \\ 2010 / 10 / 18 & 01: 11: 35.781 & 33.185 & -115.569 & 3.7 \\ 2010 / 10 / 18 & 20: 04: 14.781 & 33.164 & -115.640 & 3.4 \\ 2010 / 10 / 18 & 20: 05: 40.781 & 33.205 & -115.577 & 0.0 \\ 2010 / 10 / 18 & 20: 33: 43.781 & 33.197 & -115.596 & 3.0 \\ 2010 / 10 / 18 & 22: 34: 27.781 & 33.165 & -115.623 & 7.0 \\ 2010 / 10 / 18 & 22: 41: 19.781 & 33.165 & -115.623 & 7.0 \\ 2010 / 10 / 19 & 00: 02: 23.781 & 33.176 & -115.604 & 0.5 \\ 2010 / 10 / 19 & 00: 03: 59.781 & 33.182 & -115.602 & 2.5 \\ 2010 / 10 / 19 & 00: 10: 25.781 & 33.176 & -115.609 & 2.1 \\ 2010 / 10 / 19 & 00: 10: 49.781 & 33.179 & -115.594 & 1.5 \\ 2010 / 10 / 19 & 00: 15: 24.781 & 33.176 & -115.608 & 0.4 \\ 2010 / 10 / 19 & 00: 18: 11.781 & 33.182 & -115.602 & 2.5 \\ 2010 / 10 / 19 & 00: 18: 48.781 & 33.214 & -115.620 & 5.3 \\ 2010 / 10 / 19 & 00: 20: 50.781 & 33.176 & -115.608 & 0.4 \\ 2010 / 10 / 19 & 00: 26: 03.781 & 33.179 & -115.594 & 1.5 \\ 2010 / 10 / 19 & 00: 28: 26.781 & 33.176 & -115.609 & 2.1 \\ 2010 / 10 / 19 & 00: 30: 52.781 & 33.182 & -115.606 & 1.7\end{array}$




$\begin{array}{lllll}2010 / 10 / 19 & 00: 31: 24.781 & 33.130 & -115.634 & 0.0 \\ 2010 / 10 / 19 & 03: 12: 21.781 & 33.157 & -115.668 & 0.1 \\ 2010 / 10 / 19 & 03: 13: 05.781 & 33.157 & -115.668 & 0.1 \\ 2010 / 10 / 19 & 03: 26: 58.781 & 33.157 & -115.668 & 0.1 \\ 2010 / 10 / 19 & 03: 27: 46.781 & 33.157 & -115.668 & 0.1 \\ 2010 / 10 / 19 & 03: 31: 18.781 & 33.157 & -115.668 & 0.1 \\ 2010 / 10 / 19 & 03: 36: 34.781 & 33.157 & -115.668 & 0.1 \\ 2010 / 10 / 19 & 03: 37: 04.781 & 33.157 & -115.668 & 0.1 \\ 2010 / 10 / 19 & 05: 02: 16.781 & 33.177 & -115.614 & 1.2 \\ 2010 / 10 / 20 & 01: 17: 00.781 & 33.158 & -115.644 & 0.1 \\ 2010 / 10 / 20 & 01: 31: 30.781 & 33.158 & -115.644 & 0.1 \\ 2010 / 10 / 20 & 01: 43: 26.781 & 33.181 & -115.629 & 4.0 \\ 2010 / 10 / 20 & 02: 26: 10.781 & 33.190 & -115.574 & 3.1 \\ 2010 / 10 / 20 & 03: 58: 25.781 & 33.108 & -115.602 & 8.1 \\ 2010 / 10 / 20 & 04: 11: 51.781 & 33.182 & -115.602 & 2.5 \\ 2010 / 10 / 20 & 05: 33: 55.781 & 33.179 & -115.594 & 1.5 \\ 2010 / 10 / 20 & 17: 38: 58.781 & 33.118 & -115.610 & 9.1 \\ 2010 / 10 / 20 & 18: 04: 56.781 & 33.229 & -115.645 & 5.7 \\ 2010 / 10 / 20 & 18: 07: 22.781 & 33.209 & -115.590 & 3.1 \\ 2010 / 10 / 20 & 18: 07: 36.781 & 33.213 & -115.584 & 1.3 \\ 2010 / 10 / 20 & 18: 09: 36.781 & 33.176 & -115.606 & 6.3 \\ 2010 / 10 / 20 & 18: 11: 49.781 & 33.192 & -115.572 & 1.6 \\ 2010 / 10 / 20 & 19: 02: 42.781 & 33.196 & -115.618 & 7.3 \\ 2010 / 10 / 20 & 20: 50: 00.781 & 33.192 & -115.572 & 1.6 \\ 2010 / 10 / 22 & 04: 03: 22.781 & 33.176 & -115.618 & 3.7 \\ 2010 / 10 / 22 & 05: 44: 39.781 & 33.183 & -115.617 & 4.3 \\ 2010 / 10 / 22 & 11: 49: 08.781 & 33.172 & -115.611 & 3.7 \\ 2010 / 10 / 22 & 14: 38: 30.781 & 33.157 & -115.680 & 0.2 \\ 2010 / 10 / 23 & 09: 21: 51.781 & 33.157 & -115.656 & 2.4 \\ 2010 / 10 / 23 & 09: 36: 04.781 & 33.157 & -115.656 & 2.4 \\ 2010 / 10 / 24 & 02: 22: 53.781 & 33.187 & -115.622 & 4.0 \\ 2010 / 10 / 24 & 04: 34: 54.781 & 33.174 & -115.604 & 0.1 \\ 2010 / 10 / 24 & 10: 24: 42.781 & 33.108 & -115.602 & 8.1 \\ 2010 / 10 / 24 & 13: 46: 15.781 & 33.140 & -115.636 & 7.3 \\ 2010 / 10 / 24 & 15: 12: 52.781 & 33.108 & -115.602 & 8.1 \\ 2010 / 10 / 24 & 15: 14: 03.781 & 33.108 & -115.602 & 8.1 \\ 2010 / 10 / 24 & 22: 24: 19.781 & 33.173 & -115.650 & 1.8 \\ 2010 / 10 / 24 & 22: 34: 44.781 & 33.163 & -115.637 & 0.1 \\ 2010 / 10 / 25 & 04: 17: 50.781 & 33.190 & -115.608 & 2.2 \\ 2010 / 10 / 25 & 10: 03: 35.781 & 33.193 & -115.583 & 5.9 \\ 2010 / 10 / 25 & 16: 45: 58.781 & 33.199 & -115.571 & 3.3 \\ 2010 / 10 / 26 & 03: 44: 09.781 & 33.218 & -115.639 & 3.0 \\ 2010 / 10 / 26 & 07: 43: 18.781 & 33.108 & -115.626 & 0.6 \\ 26 & 08: 22: 24.781 & 33.176 & -115.602 & 8.1 \\ 26 & 08: 26: 47.781 & 33.188 & -115.573 & 0.2\end{array}$




$\begin{array}{lllll}2010 / 10 / 26 & 08: 39: 33.781 & 33.197 & -115.659 & 6.4 \\ 2010 / 10 / 26 & 08: 44: 48.781 & 33.197 & -115.659 & 6.4 \\ 2010 / 10 / 26 & 20: 42: 02.781 & 33.157 & -115.680 & 0.2 \\ 2010 / 10 / 26 & 21: 15: 09.781 & 33.157 & -115.680 & 0.2 \\ 2010 / 10 / 27 & 07: 05: 14.781 & 33.170 & -115.654 & 0.0 \\ 2010 / 10 / 27 & 07: 34: 35.781 & 33.170 & -115.654 & 0.0 \\ 2010 / 10 / 27 & 07: 56: 54.781 & 33.170 & -115.654 & 0.0 \\ 2010 / 10 / 27 & 14: 28: 33.781 & 33.199 & -115.570 & 0.2 \\ 2010 / 10 / 27 & 19: 03: 28.781 & 33.202 & -115.575 & 3.8 \\ 2010 / 10 / 27 & 19: 04: 33.781 & 33.159 & -115.641 & 0.9 \\ 2010 / 10 / 27 & 19: 37: 09.781 & 33.108 & -115.602 & 8.1 \\ 2010 / 10 / 28 & 06: 12: 34.641 & 33.177 & -115.606 & 0.1 \\ 2010 / 10 / 28 & 06: 27: 07.641 & 33.180 & -115.609 & 0.0 \\ 2010 / 10 / 28 & 06: 28: 38.641 & 33.177 & -115.606 & 0.1 \\ 2010 / 10 / 28 & 06: 29: 56.641 & 33.177 & -115.606 & 0.1 \\ 2010 / 10 / 28 & 06: 32: 13.641 & 33.177 & -115.606 & 0.1 \\ 2010 / 10 / 28 & 06: 35: 18.641 & 33.181 & -115.608 & 0.0 \\ 2010 / 10 / 28 & 08: 12: 30.641 & 33.108 & -115.602 & 8.1 \\ 2010 / 10 / 28 & 08: 15: 41.641 & 33.135 & -115.644 & 6.9 \\ 2010 / 10 / 28 & 08: 16: 59.641 & 33.161 & -115.644 & 1.0 \\ 2010 / 10 / 28 & 09: 38: 46.641 & 33.178 & -115.632 & 2.3 \\ 2010 / 10 / 28 & 12: 02: 38.641 & 33.176 & -115.609 & 2.1 \\ 2010 / 10 / 28 & 18: 36: 27.641 & 33.188 & -115.573 & 0.2 \\ 2010 / 10 / 28 & 18: 37: 59.641 & 33.187 & -115.604 & 3.2 \\ 2010 / 10 / 28 & 21: 26: 38.641 & 33.176 & -115.608 & 0.4 \\ 2010 / 10 / 28 & 22: 35: 00.641 & 33.176 & -115.604 & 0.5 \\ 2010 / 10 / 29 & 00: 30: 11.641 & 33.176 & -115.608 & 0.4 \\ 2010 / 10 / 29 & 09: 57: 51.641 & 33.187 & -115.622 & 4.0 \\ 2010 / 10 / 29 & 09: 59: 31.641 & 33.188 & -115.609 & 2.3 \\ 2010 / 10 / 29 & 10: 09: 00.641 & 33.187 & -115.622 & 4.0 \\ 2010 / 10 / 29 & 10: 13: 23.641 & 33.188 & -115.599 & 0.8 \\ 2010 / 10 / 29 & 10: 36: 04.641 & 33.190 & -115.608 & 2.2 \\ 2010 / 10 / 29 & 13: 13: 06.641 & 33.178 & -115.611 & 1.6 \\ 2010 / 10 / 29 & 18: 31: 09.641 & 33.140 & -115.636 & 7.3 \\ 2010 / 10 / 29 & 20: 00: 06.641 & 33.108 & -115.602 & 8.1 \\ 2010 / 10 / 29 & 20: 41: 25.641 & 33.175 & -115.601 & 0.1 \\ 2010 / 10 / 29 & 20: 47: 55.641 & 33.189 & -115.576 & 0.1 \\ 2010 / 10 / 29 & 20: 49: 29.641 & 33.178 & -115.611 & 1.6 \\ 2010 / 10 / 29 & 21: 45: 45.641 & 33.176 & -115.609 & 2.1 \\ 2010 / 10 / 30 & 23: 39: 59.641 & 33.108 & -115.602 & 8.1 \\ 2010 / 10 / 30 / 30 & 10: 20: 29.641 & 33.179 & -115.614 & 1.8 \\ 2010 / 30 & 18: 09: 51.641 & 33.175 & -115.601 & 0.1 \\ 20 & 18: 10: 40.641 & 33.175 & -115.601 & 0.1 \\ 20: 11: 02.641 & 33.208 & -115.582 & 2.5\end{array}$




$\begin{array}{lllll}2010 / 10 / 30 & 22: 13: 16.641 & 33.117 & -115.613 & 7.9 \\ 2010 / 10 / 31 & 00: 39: 05.641 & 33.183 & -115.607 & 0.0 \\ 2010 / 10 / 31 & 00: 46: 32.641 & 33.177 & -115.606 & 0.1 \\ 2010 / 10 / 31 & 07: 02: 36.641 & 33.181 & -115.665 & 4.2 \\ 2010 / 10 / 31 & 19: 13: 01.641 & 33.192 & -115.572 & 1.6 \\ 2010 / 11 / 01 & 00: 24: 26.641 & 33.226 & -115.588 & 4.6 \\ 2010 / 11 / 01 & 11: 56: 45.641 & 33.159 & -115.635 & 7.3 \\ 2010 / 11 / 01 & 20: 02: 18.641 & 33.117 & -115.613 & 7.9 \\ 2010 / 11 / 02 & 04: 35: 17.641 & 33.185 & -115.608 & 2.0 \\ 2010 / 11 / 02 & 04: 38: 48.641 & 33.193 & -115.583 & 5.9 \\ 2010 / 11 / 02 & 04: 39: 54.641 & 33.187 & -115.604 & 3.2 \\ 2010 / 11 / 02 & 05: 57: 51.641 & 33.184 & -115.604 & 0.1 \\ 2010 / 11 / 02 & 15: 15: 23.641 & 33.181 & -115.665 & 4.2 \\ 2010 / 11 / 03 & 03: 57: 52.641 & 33.208 & -115.582 & 2.5 \\ 2010 / 11 / 03 & 11: 40: 43.641 & 33.177 & -115.606 & 0.1 \\ 2010 / 11 / 03 & 13: 49: 41.641 & 33.117 & -115.613 & 7.9 \\ 2010 / 11 / 04 & 01: 03: 27.641 & 33.205 & -115.577 & 0.0 \\ 2010 / 11 / 04 & 06: 41: 06.641 & 33.143 & -115.638 & 4.8 \\ 2010 / 11 / 04 & 12: 12: 14.641 & 33.174 & -115.604 & 0.1 \\ 2010 / 11 / 04 & 12: 12: 57.641 & 33.135 & -115.644 & 6.9 \\ 2010 / 11 / 04 & 17: 57: 26.641 & 33.203 & -115.578 & 2.2 \\ 2010 / 11 / 04 & 19: 01: 56.641 & 33.118 & -115.590 & 13.8 \\ 2010 / 11 / 04 & 19: 02: 53.641 & 33.160 & -115.633 & 4.6 \\ 2010 / 11 / 04 & 19: 09: 19.641 & 33.100 & -115.590 & 14.6 \\ 2010 / 11 / 05 & 03: 44: 17.641 & 33.186 & -115.614 & 2.4 \\ 2010 / 11 / 05 & 04: 52: 55.641 & 33.231 & -115.605 & 4.8 \\ 2010 / 11 / 05 & 04: 55: 45.641 & 33.157 & -115.668 & 0.1 \\ 2010 / 11 / 05 & 09: 44: 19.641 & 33.190 & -115.604 & 1.2 \\ 2010 / 11 / 05 & 09: 51: 18.641 & 33.179 & -115.594 & 1.5 \\ 2010 / 11 / 05 & 11: 38: 26.641 & 33.176 & -115.604 & 0.0 \\ 2010 / 11 / 06 & 00: 38: 02.641 & 33.193 & -115.615 & 3.9 \\ 2010 / 11 / 06 & 02: 21: 00.641 & 33.188 & -115.609 & 2.3 \\ 2010 / 11 / 06 & 05: 53: 03.641 & 33.179 & -115.614 & 1.8 \\ 2010 / 11 / 06 & 05: 54: 24.641 & 33.179 & -115.614 & 1.8 \\ 2010 / 11 / 06 & 05: 56: 38.641 & 33.179 & -115.614 & 1.8 \\ 2010 / 11 / 06 & 05: 57: 13.641 & 33.179 & -115.614 & 1.8 \\ 2010 / 11 / 06 & 05: 59: 12.641 & 33.202 & -115.575 & 3.8 \\ 2010 / 11 / 06 & 05: 59: 32.641 & 33.208 & -115.582 & 2.5 \\ 2010 / 11 / 06 & 06: 00: 14.641 & 33.208 & -115.582 & 2.5 \\ 2010 / 11 / 06 & 06: 01: 02.641 & 33.179 & -115.614 & 1.8 \\ 2010 / 11 / 06 & 07: 05: 00.641 & 33.179 & -115.614 & 1.8 \\ 2010 / 11 / 06 & 20: 07: 34.641 & 33.174 & -115.604 & 0.1 \\ 2010 / 11 / 07 & 01: 11: 56.641 & 33.196 & -115.587 & 5.3 \\ 2010 / 11 / 07 & 01: 16: 31.641 & 33.196 & -115.587 & 5.3 \\ 2010 / 11 / 08 & 03: 33: 15.641 & 33.196 & -115.587 & 5.3 \\ & & -115.665 & 4.2\end{array}$




\begin{tabular}{lllll}
$2010 / 11 / 08$ & $04: 11: 31.641$ & 33.181 & -115.665 & 4.2 \\
$2010 / 11 / 08$ & $09: 49: 05.641$ & 33.184 & -115.611 & 2.7 \\
$2010 / 11 / 08$ & $09: 49: 56.641$ & 33.184 & -115.611 & 2.7 \\
$2010 / 11 / 08$ & $13: 22: 58.641$ & 33.158 & -115.638 & 0.4 \\
$2010 / 11 / 08$ & $13: 23: 50.641$ & 33.158 & -115.638 & 0.4 \\
$2010 / 11 / 08$ & $13: 24: 48.641$ & 33.158 & -115.638 & 0.4 \\
$2010 / 11 / 08$ & $13: 25: 08.641$ & 33.159 & -115.640 & 1.5 \\
$2010 / 11 / 08$ & $18: 45: 20.641$ & 33.177 & -115.606 & 0.1 \\
$2010 / 11 / 10$ & $01: 16: 52.641$ & 33.198 & -115.571 & 0.2 \\
$2010 / 11 / 10$ & $05: 59: 50.641$ & 33.192 & -115.572 & 1.6 \\
$2010 / 11 / 10$ & $07: 02: 09.641$ & 33.190 & -115.612 & 1.0 \\
$2010 / 11 / 10$ & $07: 02: 35.641$ & 33.190 & -115.612 & 1.0 \\
$2010 / 11 / 10$ & $07: 08: 19.641$ & 33.174 & -115.604 & 0.1 \\
$2010 / 11 / 10$ & $07: 23: 29.641$ & 33.229 & -115.645 & 5.7 \\
$2010 / 11 / 10$ & $09: 40: 30.641$ & 33.231 & -115.655 & 4.8 \\
$2010 / 11 / 10$ & $10: 09: 13.641$ & 33.175 & -115.601 & 0.1 \\
$2010 / 11 / 10$ & $14: 26: 30.641$ & 33.140 & -115.636 & 7.3 \\
$2010 / 11 / 10$ & $15: 10: 38.641$ & 33.199 & -115.617 & 0.0 \\
$2010 / 11 / 10$ & $20: 02: 18.641$ & 33.135 & -115.644 & 6.9 \\
$2010 / 11 / 11$ & $01: 51: 07.641$ & 33.199 & -115.617 & 0.0 \\
$2010 / 11 / 11$ & $05: 55: 04.641$ & 33.173 & -115.610 & 1.3 \\
$2010 / 11 / 11$ & $19: 32: 16.641$ & 33.180 & -115.609 & 0.0 \\
$2010 / 11 / 11$ & $19: 53: 44.641$ & 33.180 & -115.609 & 0.0 \\
$2010 / 11 / 12$ & $05: 13: 10.641$ & 33.108 & -115.602 & 8.1 \\
$2010 / 11 / 12$ & $11: 16: 35.641$ & 33.183 & -115.617 & 4.3 \\
$2010 / 11 / 12$ & $11: 17: 37.641$ & 33.177 & -115.622 & 4.2 \\
$2010 / 11 / 12$ & $11: 18: 56.641$ & 33.177 & -115.622 & 4.2 \\
$2010 / 11 / 12$ & $11: 22: 40.641$ & 33.177 & -115.622 & 4.2 \\
$2010 / 11 / 12$ & $11: 23: 53.641$ & 33.177 & -115.622 & 4.2 \\
$2010 / 11 / 12$ & $11: 24: 17.641$ & 33.177 & -115.622 & 4.2 \\
$2010 / 11 / 12$ & $11: 25: 02.641$ & 33.177 & -115.622 & 4.2 \\
$2010 / 11 / 12$ & $11: 25: 57.641$ & 33.177 & -115.622 & 4.2 \\
$2010 / 11 / 12$ & $11: 26: 54.641$ & 33.177 & -115.622 & 4.2 \\
$2010 / 11 / 12$ & $11: 27: 11.641$ & 33.177 & -115.622 & 4.2 \\
$2010 / 11 / 12$ & $11: 27: 58.641$ & 33.177 & -115.622 & 4.2 \\
$2010 / 11 / 12$ & $11: 28: 23.641$ & 33.161 & -115.640 & 0.1 \\
$2010 / 11 / 12$ & $11: 28: 44.641$ & 33.177 & -115.622 & 4.2 \\
$2010 / 11 / 12$ & $11: 28: 55.641$ & 33.177 & -115.622 & 4.2 \\
$2010 / 11 / 12$ & $11: 29: 30.641$ & 33.161 & -115.640 & 0.1 \\
$2010 / 11 / 12$ & $11: 30: 19.641$ & 33.161 & -115.640 & 0.1 \\
$2010 / 11 / 12$ & $11: 30: 52.641$ & 33.177 & -115.622 & 4.2 \\
$2011 / 11 / 12$ & $11: 32: 23.641$ & 33.177 & -115.640 & 0.1 \\
\hline & $11: 33: 06.641$ & 33.190 & -115.622 & 4.2 \\
2012 & $11: 34: 45.641$ & 33.160 & -115.633 & 4.6
\end{tabular}




$\begin{array}{lllll}2010 / 11 / 12 & 11: 35: 19.641 & 33.160 & -115.633 & 4.6 \\ 2010 / 11 / 12 & 11: 36: 06.641 & 33.160 & -115.633 & 4.6 \\ 2010 / 11 / 12 & 11: 36: 42.641 & 33.160 & -115.633 & 4.6 \\ 2010 / 11 / 12 & 11: 37: 08.641 & 33.160 & -115.633 & 4.6 \\ 2010 / 11 / 12 & 11: 37: 48.641 & 33.164 & -115.632 & 2.6 \\ 2010 / 11 / 12 & 11: 38: 22.641 & 33.160 & -115.633 & 4.6 \\ 2010 / 11 / 12 & 11: 38: 44.641 & 33.161 & -115.640 & 0.1 \\ 2010 / 11 / 12 & 11: 39: 40.641 & 33.177 & -115.622 & 4.2 \\ 2010 / 11 / 12 & 11: 40: 36.641 & 33.186 & -115.612 & 0.6 \\ 2010 / 11 / 12 & 11: 42: 38.641 & 33.160 & -115.633 & 4.6 \\ 2010 / 11 / 12 & 11: 43: 50.641 & 33.160 & -115.633 & 4.6 \\ 2010 / 11 / 12 & 11: 49: 41.641 & 33.160 & -115.633 & 4.6 \\ 2010 / 11 / 12 & 11: 50: 25.641 & 33.160 & -115.633 & 4.6 \\ 2010 / 11 / 12 & 11: 51: 31.641 & 33.177 & -115.622 & 4.2 \\ 2010 / 11 / 12 & 11: 52: 20.641 & 33.193 & -115.615 & 3.9 \\ 2010 / 11 / 12 & 11: 52: 33.641 & 33.177 & -115.622 & 4.2 \\ 2010 / 11 / 12 & 11: 54: 30.641 & 33.100 & -115.590 & 14.6 \\ 2010 / 11 / 12 & 11: 57: 22.641 & 33.165 & -115.623 & 7.0 \\ 2010 / 11 / 12 & 12: 13: 18.641 & 33.190 & -115.612 & 1.0 \\ 2010 / 11 / 12 & 12: 19: 45.641 & 33.160 & -115.633 & 4.6 \\ 2010 / 11 / 12 & 12: 54: 32.641 & 33.176 & -115.604 & 0.5 \\ 2010 / 11 / 12 & 12: 55: 27.641 & 33.164 & -115.632 & 2.6 \\ 2010 / 11 / 12 & 13: 10: 55.641 & 33.181 & -115.608 & 0.0 \\ 2010 / 11 / 12 & 13: 11: 06.641 & 33.181 & -115.608 & 0.0 \\ 2010 / 11 / 12 & 15: 06: 42.641 & 33.177 & -115.622 & 4.2 \\ 2010 / 11 / 12 & 15: 23: 15.641 & 33.108 & -115.602 & 8.1 \\ 2010 / 11 / 12 & 17: 14: 26.641 & 33.170 & -115.626 & 0.6 \\ 2010 / 11 / 12 & 17: 15: 04.641 & 33.135 & -115.644 & 6.9 \\ 2010 / 11 / 12 & 17: 19: 44.641 & 33.158 & -115.638 & 0.4 \\ 2010 / 11 / 12 & 17: 22: 32.641 & 33.115 & -115.585 & 6.5 \\ 2010 / 11 / 12 & 17: 33: 30.641 & 33.158 & -115.638 & 0.4 \\ 2010 / 11 / 12 & 17: 33: 52.641 & 33.135 & -115.644 & 6.9 \\ 2010 / 11 / 12 & 17: 35: 29.641 & 33.158 & -115.638 & 0.4 \\ 2010 / 11 / 12 & 18: 11: 24.641 & 33.135 & -115.644 & 6.9 \\ 2010 / 11 / 12 & 18: 12: 29.641 & 33.149 & -115.640 & 3.5 \\ 2010 / 11 / 12 & 18: 12: 46.641 & 33.158 & -115.638 & 0.4 \\ 2010 / 11 / 12 & 18: 25: 03.641 & 33.158 & -115.642 & 0.1 \\ 2010 / 11 / 12 & 19: 24: 09.641 & 33.158 & -115.638 & 0.4 \\ 2010 / 11 / 13 & 01: 15: 18.641 & 33.181 & -115.629 & 4.0 \\ 2010 / 11 / 13 & 07: 50: 35.641 & 33.147 & -115.623 & 7.9 \\ 2010 / 11 / 13 & 11: 01: 51.641 & 33.158 & -115.638 & 0.4 \\ 2010 / 11 / 13 & 11: 05: 37.641 & 33.158 & -115.638 & 0.4 \\ 2010 / 11 / 13 & 16: 15: 15.641 & 33.157 & -115.680 & 0.2 \\ 2010 / 11 / 13 & 16: 38: 33.641 & 33.157 & -115.680 & 0.2 \\ 2010 / 11 / 13 & 16: 39: 23.641 & 33.157 & -115.680 & 0.2 \\ 2010 / 11 / 13 & 17: 00: 31.641 & 33.142 & -115.628 & 10.3\end{array}$




$\begin{array}{lllll}2010 / 11 / 13 & 17: 37: 13.641 & 33.108 & -115.602 & 8.1 \\ 2010 / 11 / 13 & 17: 47: 16.641 & 33.177 & -115.622 & 4.2 \\ 2010 / 11 / 14 & 04: 59: 11.641 & 33.181 & -115.603 & 1.4 \\ 2010 / 11 / 14 & 09: 38: 29.641 & 33.160 & -115.633 & 4.6 \\ 2010 / 11 / 14 & 10: 13: 51.641 & 33.179 & -115.614 & 1.8 \\ 2010 / 11 / 14 & 10: 14: 35.641 & 33.193 & -115.615 & 3.9 \\ 2010 / 11 / 14 & 10: 15: 02.641 & 33.193 & -115.615 & 3.9 \\ 2010 / 11 / 14 & 10: 16: 03.641 & 33.193 & -115.615 & 3.9 \\ 2010 / 11 / 14 & 10: 17: 02.641 & 33.192 & -115.572 & 1.6 \\ 2010 / 11 / 14 & 15: 32: 58.641 & 33.108 & -115.602 & 8.1 \\ 2010 / 11 / 14 & 16: 50: 39.641 & 33.164 & -115.632 & 2.6 \\ 2010 / 11 / 14 & 17: 00: 47.641 & 33.164 & -115.632 & 2.6 \\ 2010 / 11 / 14 & 17: 06: 29.641 & 33.196 & -115.587 & 5.3 \\ 2010 / 11 / 14 & 18: 28: 11.641 & 33.161 & -115.644 & 1.0 \\ 2010 / 11 / 14 & 19: 23: 25.641 & 33.141 & -115.641 & 4.5 \\ 2010 / 11 / 15 & 00: 20: 28.641 & 33.181 & -115.611 & 0.4 \\ 2010 / 11 / 15 & 01: 03: 32.641 & 33.149 & -115.640 & 3.5 \\ 2010 / 11 / 15 & 22: 20: 39.641 & 33.108 & -115.602 & 8.1 \\ 2010 / 11 / 15 & 23: 50: 24.641 & 33.184 & -115.609 & 2.2 \\ 2010 / 11 / 15 & 23: 51: 19.641 & 33.176 & -115.608 & 0.4 \\ 2010 / 11 / 15 & 23: 59: 49.641 & 33.184 & -115.609 & 2.2 \\ 2010 / 11 / 16 & 02: 19: 38.641 & 33.181 & -115.601 & 0.7 \\ 2010 / 11 / 16 & 10: 58: 33.641 & 33.176 & -115.604 & 0.5 \\ 2010 / 11 / 16 & 12: 18: 40.641 & 33.117 & -115.613 & 7.9 \\ 2010 / 11 / 16 & 15: 33: 59.641 & 33.154 & -115.672 & 0.3 \\ 2010 / 11 / 16 & 15: 34: 19.641 & 33.157 & -115.656 & 2.4 \\ 2010 / 11 / 16 & 15: 34: 46.641 & 33.161 & -115.644 & 1.0 \\ 2010 / 11 / 16 & 15: 36: 40.641 & 33.157 & -115.680 & 0.2 \\ 2010 / 11 / 16 & 15: 37: 50.641 & 33.155 & -115.663 & 2.3 \\ 2010 / 11 / 16 & 15: 38: 28.641 & 33.108 & -115.602 & 8.1 \\ 2010 / 11 / 16 & 16: 33: 37.641 & 33.140 & -115.636 & 7.3 \\ 2010 / 11 / 16 & 21: 38: 47.641 & 33.140 & -115.636 & 7.3 \\ 2010 / 11 / 16 & 21: 45: 49.641 & 33.163 & -115.637 & 0.1 \\ 2010 / 11 / 16 & 21: 49: 22.641 & 33.163 & -115.637 & 0.1 \\ 2010 / 11 / 16 & 21: 54: 35.641 & 33.161 & -115.644 & 0.3 \\ 2010 / 11 / 17 & 01: 24: 14.641 & 33.117 & -115.613 & 7.9 \\ 2010 / 11 / 17 & 09: 15: 23.641 & 33.160 & -115.633 & 4.6 \\ 2010 / 11 / 17 & 09: 15: 39.641 & 33.160 & -115.633 & 4.6 \\ 2010 / 11 / 17 & 09: 16: 42.641 & 33.160 & -115.633 & 4.6 \\ 2010 / 11 / 177 & 12: 12: 32.641 & 33.160 & -115.633 & 4.6 \\ 2010 / 11 / 17 & 13: 10: 07.641 & 33.160 & -115.633 & 4.6 \\ 2011 / 17 & 15: 10: 04.641 & 33.184 & -115.633 & 4.6 \\ 20: 50.641 & 33.184 & -115.601 & 3.5 \\ 20.601 & 3.5 \\ 203: 29: 23.641 & 33.187 & -115.622 & 4.0\end{array}$




$\begin{array}{lllll}2010 / 11 / 17 & 21: 55: 09.641 & 33.199 & -115.570 & 0.2 \\ 2010 / 11 / 17 & 21: 56: 15.641 & 33.199 & -115.570 & 0.2 \\ 2010 / 11 / 17 & 22: 55: 04.641 & 33.184 & -115.604 & 0.1 \\ 2010 / 11 / 17 & 22: 56: 18.641 & 33.179 & -115.614 & 1.8 \\ 2010 / 11 / 17 & 22: 56: 42.641 & 33.179 & -115.614 & 1.8 \\ 2010 / 11 / 17 & 23: 00: 46.641 & 33.179 & -115.614 & 1.8 \\ 2010 / 11 / 17 & 23: 05: 26.641 & 33.184 & -115.609 & 2.2 \\ 2010 / 11 / 17 & 23: 06: 38.641 & 33.184 & -115.609 & 2.2 \\ 2010 / 11 / 18 & 07: 42: 26.641 & 33.140 & -115.636 & 7.3 \\ 2010 / 11 / 18 & 07: 43: 06.641 & 33.140 & -115.636 & 7.3 \\ 2010 / 11 / 18 & 11: 41: 01.641 & 33.135 & -115.644 & 6.9 \\ 2010 / 11 / 19 & 04: 40: 43.641 & 33.176 & -115.609 & 2.1 \\ 2010 / 11 / 19 & 10: 58: 54.641 & 33.100 & -115.590 & 14.6 \\ 2010 / 11 / 19 & 13: 06: 55.641 & 33.108 & -115.602 & 8.1 \\ 2010 / 11 / 19 & 23: 02: 22.641 & 33.140 & -115.636 & 7.3 \\ 2010 / 11 / 19 & 23: 30: 38.641 & 33.181 & -115.665 & 4.2 \\ 2010 / 11 / 20 & 10: 00: 26.641 & 33.117 & -115.613 & 7.9 \\ 2010 / 11 / 20 & 12: 18: 35.641 & 33.176 & -115.608 & 0.4 \\ 2010 / 11 / 20 & 12: 29: 30.641 & 33.108 & -115.602 & 8.1 \\ 2010 / 11 / 20 & 22: 27: 52.641 & 33.202 & -115.671 & 6.3 \\ 2010 / 11 / 20 & 23: 37: 11.641 & 33.176 & -115.609 & 2.1 \\ 2010 / 11 / 21 & 00: 28: 43.641 & 33.100 & -115.590 & 14.6 \\ 2010 / 11 / 21 & 02: 54: 30.641 & 33.183 & -115.599 & 0.1 \\ 2010 / 11 / 21 & 05: 14: 32.641 & 33.148 & -115.633 & 4.3 \\ 2010 / 11 / 21 & 05: 30: 41.641 & 33.100 & -115.590 & 14.6 \\ 2010 / 11 / 21 & 06: 50: 06.641 & 33.177 & -115.606 & 0.1 \\ 2010 / 11 / 22 & 06: 00: 12.641 & 33.108 & -115.602 & 8.1 \\ 2010 / 11 / 22 & 11: 52: 05.641 & 33.170 & -115.600 & 1.1 \\ 2010 / 11 / 22 & 15: 54: 50.641 & 33.201 & -115.544 & 3.4 \\ 2010 / 11 / 22 & 15: 55: 22.641 & 33.188 & -115.573 & 0.2 \\ 2010 / 11 / 23 & 06: 04: 43.641 & 33.172 & -115.610 & 2.2 \\ 2010 / 11 / 23 & 06: 25: 27.641 & 33.189 & -115.605 & 1.5 \\ 2010 / 11 / 23 & 14: 28: 30.641 & 33.108 & -115.602 & 8.1 \\ 2010 / 11 / 24 & 06: 36: 38.641 & 33.183 & -115.599 & 0.1 \\ 2010 / 11 / 24 & 06: 36: 58.641 & 33.183 & -115.599 & 0.1 \\ 2010 / 11 / 24 & 06: 49: 41.641 & 33.183 & -115.599 & 0.1 \\ 2010 / 11 / 24 & 07: 27: 10.641 & 33.173 & -115.609 & 0.1 \\ 2010 / 11 / 24 & 09: 41: 12.641 & 33.196 & -115.573 & 0.1 \\ 2010 / 11 / 24 & 23: 03: 18.641 & 33.181 & -115.612 & 1.1 \\ 2010 / 11 / 24 & 23: 05: 07.641 & 33.176 & -115.609 & 2.1 \\ 2010 / 11 / 24 & 23: 06: 02.641 & 33.179 & -115.594 & 1.5 \\ 2010 / 11 / 25 & 00: 11: 57.641 & 33.184 & -115.597 & 0.1 \\ 2010 / 11 / 25 & 08: 15: 15.641 & 33.170 & -115.600 & 1.1 \\ 2010 / 11 / 25 & 13: 55: 15.641 & 33.195 & -115.572 & 0.1 \\ 2010 / 11 / 26 & 06: 47: 48.641 & 33.161 & -115.651 & 0.7 \\ 2010 / 11 / 26 & 08: 24: 19.641 & 33.176 & -115.606 & 6.3\end{array}$




$\begin{array}{lllll}2010 / 11 / 26 & 10: 58: 01.641 & 33.118 & -115.610 & 9.1 \\ 2010 / 11 / 26 & 13: 01: 28.641 & 33.164 & -115.668 & 0.0 \\ 2010 / 11 / 26 & 13: 11: 14.641 & 33.157 & -115.656 & 2.4 \\ 2010 / 11 / 26 & 13: 22: 00.641 & 33.161 & -115.651 & 0.7 \\ 2010 / 11 / 26 & 17: 30: 52.641 & 33.186 & -115.593 & 0.1 \\ 2010 / 11 / 26 & 20: 51: 37.641 & 33.177 & -115.606 & 0.1 \\ 2010 / 11 / 26 & 20: 57: 20.641 & 33.176 & -115.604 & 0.5 \\ 2010 / 11 / 26 & 21: 06: 16.641 & 33.177 & -115.621 & 2.7 \\ 2010 / 11 / 26 & 22: 08: 33.641 & 33.188 & -115.599 & 0.8 \\ 2010 / 11 / 26 & 23: 52: 57.641 & 33.176 & -115.604 & 0.5 \\ 2010 / 11 / 27 & 03: 02: 20.641 & 33.175 & -115.601 & 0.1 \\ 2010 / 11 / 27 & 04: 07: 37.641 & 33.100 & -115.590 & 14.6 \\ 2010 / 11 / 27 & 04: 07: 53.641 & 33.100 & -115.590 & 14.6 \\ 2010 / 11 / 27 & 04: 14: 15.641 & 33.149 & -115.640 & 3.5 \\ 2010 / 11 / 27 & 04: 38: 19.641 & 33.157 & -115.668 & 0.1 \\ 2010 / 11 / 28 & 09: 06: 39.641 & 33.182 & -115.606 & 1.7 \\ 2010 / 11 / 28 & 15: 22: 36.641 & 33.157 & -115.680 & 0.2 \\ 2010 / 11 / 28 & 15: 23: 22.641 & 33.157 & -115.680 & 0.2 \\ 2010 / 11 / 28 & 19: 37: 53.641 & 33.189 & -115.619 & 2.2 \\ 2010 / 11 / 28 & 19: 38: 36.641 & 33.189 & -115.619 & 2.2 \\ 2010 / 11 / 28 & 19: 55: 12.641 & 33.189 & -115.619 & 2.2 \\ 2010 / 11 / 28 & 22: 05: 25.641 & 33.187 & -115.608 & 0.2 \\ 2010 / 11 / 28 & 22: 52: 36.641 & 33.203 & -115.578 & 2.2 \\ 2010 / 11 / 28 & 22: 58: 57.641 & 33.187 & -115.608 & 0.2 \\ 2010 / 11 / 28 & 23: 29: 24.641 & 33.149 & -115.640 & 3.5 \\ 2010 / 11 / 28 & 23: 35: 28.641 & 33.117 & -115.613 & 7.9 \\ 2010 / 11 / 29 & 05: 49: 49.641 & 33.208 & -115.582 & 2.5 \\ 2010 / 11 / 29 & 05: 54: 24.641 & 33.186 & -115.604 & 2.0 \\ 2010 / 11 / 29 & 06: 05: 08.641 & 33.181 & -115.665 & 4.2 \\ 2010 / 11 / 29 & 11: 27: 05.641 & 33.179 & -115.594 & 1.5 \\ 2010 / 11 / 29 & 14: 54: 24.641 & 33.187 & -115.608 & 0.2 \\ 2010 / 11 / 29 & 21: 41: 29.641 & 33.214 & -115.620 & 5.3 \\ 2010 / 11 / 29 & 21: 42: 08.641 & 33.214 & -115.620 & 5.3 \\ 2010 / 11 / 29 & 22: 02: 08.641 & 33.214 & -115.620 & 5.3 \\ 2010 / 11 / 29 & 22: 02: 24.641 & 33.214 & -115.620 & 5.3 \\ 2010 / 11 / 29 & 22: 02: 44.641 & 33.201 & -115.544 & 3.4 \\ 2010 / 11 / 29 & 22: 03: 05.641 & 33.214 & -115.620 & 5.3 \\ 2010 / 11 / 29 & 22: 19: 53.641 & 33.214 & -115.620 & 5.3 \\ 2010 / 11 / 30 & 03: 23: 33.641 & 33.189 & -115.619 & 2.2 \\ 2010 / 11 / 30 & 03: 25: 12.641 & 33.189 & -115.619 & 2.2 \\ 2010 / 11 / 30 & 03: 25: 38.641 & 33.193 & -115.583 & 5.9 \\ 2010 / 11 / 30 & 03: 26: 04.641 & 33.189 & -115.619 & 2.2 \\ 2010 / 11 / 30 & 03: 26: 51.641 & 33.181 & -115.665 & 4.2 \\ 2010 / 11 / 30 & 03: 28: 07.641 & 33.189 & -115.619 & 2.2 \\ 2010 / 11 / 30 & 03: 28: 47.641 & 33.214 & -115.620 & 5.3 \\ 2010 / 11 / 30 & 03: 33: 45.641 & 33.184 & -115.609 & 2.2\end{array}$




$\begin{array}{lllll}2010 / 11 / 30 & 03: 37: 12.641 & 33.189 & -115.619 & 2.2 \\ 2010 / 11 / 30 & 03: 42: 59.641 & 33.188 & -115.573 & 0.2 \\ 2010 / 11 / 30 & 03: 47: 52.641 & 33.189 & -115.619 & 2.2 \\ 2010 / 11 / 30 & 03: 49: 40.641 & 33.192 & -115.572 & 1.6 \\ 2010 / 11 / 30 & 03: 50: 23.641 & 33.189 & -115.619 & 2.2 \\ 2010 / 11 / 30 & 03: 51: 08.641 & 33.194 & -115.583 & 3.0 \\ 2010 / 11 / 30 & 03: 56: 46.641 & 33.183 & -115.599 & 0.1 \\ 2010 / 11 / 30 & 04: 01: 39.641 & 33.193 & -115.615 & 3.9 \\ 2010 / 11 / 30 & 04: 07: 13.641 & 33.179 & -115.594 & 1.5 \\ 2010 / 11 / 30 & 04: 13: 40.641 & 33.177 & -115.621 & 2.7 \\ 2010 / 11 / 30 & 04: 14: 46.641 & 33.214 & -115.620 & 5.3 \\ 2010 / 11 / 30 & 04: 28: 06.641 & 33.186 & -115.604 & 2.0 \\ 2010 / 11 / 30 & 04: 39: 54.641 & 33.179 & -115.621 & 4.8 \\ 2010 / 11 / 30 & 05: 04: 39.641 & 33.192 & -115.572 & 1.6 \\ 2010 / 11 / 30 & 05: 16: 49.641 & 33.179 & -115.594 & 1.5 \\ 2010 / 11 / 30 & 05: 17: 46.641 & 33.189 & -115.619 & 2.2 \\ 2010 / 11 / 30 & 05: 32: 59.641 & 33.175 & -115.601 & 0.1 \\ 2010 / 11 / 30 & 12: 51: 26.641 & 33.186 & -115.624 & 2.5 \\ 2010 / 11 / 30 & 15: 49: 49.641 & 33.208 & -115.582 & 2.5 \\ 2010 / 11 / 30 & 15: 58: 38.641 & 33.108 & -115.602 & 8.1 \\ 2010 / 11 / 30 & 15: 58: 57.641 & 33.108 & -115.602 & 8.1 \\ 2010 / 11 / 30 & 16: 03: 15.641 & 33.158 & -115.642 & 0.1 \\ 2010 / 11 / 30 & 16: 03: 59.641 & 33.158 & -115.642 & 0.1 \\ 2010 / 11 / 30 & 16: 23: 33.641 & 33.176 & -115.608 & 0.4 \\ 2010 / 11 / 30 & 21: 09: 06.641 & 33.100 & -115.590 & 14.6 \\ 2010 / 11 / 30 & 21: 52: 18.641 & 33.118 & -115.610 & 9.1 \\ 2010 / 11 / 30 & 22: 16: 40.641 & 33.181 & -115.665 & 4.2 \\ 2010 / 12 / 01 & 02: 21: 50.641 & 33.175 & -115.601 & 0.1 \\ 2010 / 12 / 01 & 04: 16: 41.641 & 33.136 & -115.649 & 5.3 \\ 2010 / 12 / 01 & 08: 01: 25.641 & 33.177 & -115.606 & 0.1 \\ 2010 / 12 / 01 & 11: 55: 26.641 & 33.135 & -115.644 & 6.9 \\ 2010 / 12 / 01 & 12: 23: 46.641 & 33.115 & -115.585 & 6.5 \\ 2010 / 12 / 02 & 08: 56: 14.641 & 33.214 & -115.620 & 5.3 \\ 2010 / 12 / 02 & 23: 18: 48.641 & 33.117 & -115.613 & 7.9 \\ 2010 / 12 / 03 & 00: 31: 54.641 & 33.135 & -115.644 & 6.9 \\ 2010 / 12 / 03 & 00: 40: 25.641 & 33.142 & -115.628 & 10.3 \\ 2010 / 12 / 03 & 00: 42: 20.641 & 33.158 & -115.644 & 0.1 \\ 2010 / 12 / 03 & 00: 42: 34.641 & 33.135 & -115.644 & 6.9 \\ 2010 / 12 / 03 & 00: 43: 36.641 & 33.149 & -115.640 & 3.5 \\ 2010 / 12 / 03 & 00: 45: 23.641 & 33.158 & -115.638 & 0.4 \\ 2010 / 12 / 03 & 01: 09: 42.641 & 33.158 & -115.644 & 0.1 \\ 2010 / 12 / 03 & 06: 40: 19.641 & 33.161 & -115.651 & 0.7\end{array}$




$\begin{array}{lllll}2010 / 12 / 03 & 06: 41: 24.641 & 33.161 & -115.651 & 0.7 \\ 2010 / 12 / 03 & 06: 43: 41.641 & 33.108 & -115.602 & 8.1 \\ 2010 / 12 / 03 & 06: 44: 11.641 & 33.100 & -115.590 & 14.6 \\ 2010 / 12 / 03 & 06: 48: 27.641 & 33.161 & -115.651 & 0.7 \\ 2010 / 12 / 03 & 06: 49: 20.641 & 33.156 & -115.652 & 0.2 \\ 2010 / 12 / 03 & 06: 59: 28.641 & 33.156 & -115.652 & 0.2 \\ 2010 / 12 / 03 & 07: 10: 47.641 & 33.156 & -115.652 & 0.2 \\ 2010 / 12 / 03 & 07: 12: 00.641 & 33.161 & -115.651 & 0.7 \\ 2010 / 12 / 03 & 07: 31: 29.641 & 33.193 & -115.583 & 5.9 \\ 2010 / 12 / 04 & 04: 42: 27.641 & 33.187 & -115.608 & 0.2 \\ 2010 / 12 / 04 & 04: 42: 51.641 & 33.187 & -115.608 & 0.2 \\ 2010 / 12 / 04 & 04: 43: 38.641 & 33.186 & -115.614 & 2.4 \\ 2010 / 12 / 04 & 04: 44: 21.641 & 33.229 & -115.576 & 3.4 \\ 2010 / 12 / 04 & 04: 44: 41.641 & 33.177 & -115.621 & 2.7 \\ 2010 / 12 / 04 & 04: 44: 58.641 & 33.214 & -115.620 & 5.3 \\ 2010 / 12 / 04 & 04: 46: 00.641 & 33.188 & -115.600 & 0.1 \\ 2010 / 12 / 04 & 04: 50: 45.641 & 33.187 & -115.608 & 0.2 \\ 2010 / 12 / 04 & 04: 51: 47.641 & 33.196 & -115.587 & 5.3 \\ 2010 / 12 / 04 & 04: 53: 43.641 & 33.212 & -115.590 & 7.2 \\ 2010 / 12 / 04 & 04: 54: 15.641 & 33.184 & -115.609 & 2.2 \\ 2010 / 12 / 04 & 05: 00: 57.641 & 33.188 & -115.600 & 0.1 \\ 2010 / 12 / 04 & 05: 01: 32.641 & 33.176 & -115.609 & 2.1 \\ 2010 / 12 / 04 & 05: 02: 06.641 & 33.181 & -115.665 & 4.2 \\ 2010 / 12 / 04 & 05: 04: 01.641 & 33.187 & -115.608 & 0.2 \\ 2010 / 12 / 04 & 05: 04: 15.641 & 33.184 & -115.609 & 2.2 \\ 2010 / 12 / 04 & 05: 26: 04.641 & 33.184 & -115.609 & 2.2 \\ 2010 / 12 / 04 & 05: 27: 39.641 & 33.176 & -115.609 & 2.1 \\ 2010 / 12 / 04 & 05: 36: 54.641 & 33.187 & -115.608 & 0.2 \\ 2010 / 12 / 04 & 05: 39: 56.641 & 33.187 & -115.608 & 0.2 \\ 2010 / 12 / 04 & 05: 40: 31.641 & 33.186 & -115.614 & 2.4 \\ 2010 / 12 / 04 & 05: 42: 11.641 & 33.179 & -115.594 & 1.5 \\ 2010 / 12 / 04 & 05: 43: 09.641 & 33.226 & -115.588 & 4.6 \\ 2010 / 12 / 04 & 08: 01: 54.641 & 33.135 & -115.644 & 6.9 \\ 2010 / 12 / 04 & 10: 11: 40.641 & 33.187 & -115.608 & 0.2 \\ 2010 / 12 / 04 & 22: 54: 29.641 & 33.197 & -115.644 & 3.0 \\ 2010 / 12 / 05 & 02: 00: 25.641 & 33.176 & -115.604 & 0.5 \\ 2010 / 12 / 05 & 06: 30: 40.641 & 33.160 & -115.633 & 4.6 \\ 2010 / 12 / 05 & 06: 33: 27.641 & 33.180 & -115.603 & 0.1 \\ 2010 / 12 / 05 & 06: 33: 45.641 & 33.135 & -115.644 & 6.9 \\ 2010 / 12 / 05 & 06: 37: 53.641 & 33.135 & -115.644 & 6.9 \\ 2010 / 12 / 05 & 06: 39: 54.641 & 33.108 & -115.602 & 8.1 \\ 2010 / 12 / 05 & 06: 46: 48.641 & 33.176 & -115.609 & 2.1 \\ 2010 / 12 / 05 & 06: 48: 08.641 & 33.182 & -115.606 & 1.7 \\ 2010 / 12 / 05 & 06: 59: 46.641 & 33.173 & -115.610 & 1.3 \\ 2010 / 12 / 05 & 09: 28: 38.641 & 33.187 & -115.608 & 0.2 \\ 2010 / 12 / 05 & 09: 39: 13.641 & 33.187 & -115.608 & 0.2\end{array}$




$\begin{array}{lllll}2010 / 12 / 05 & 09: 41: 51.641 & 33.187 & -115.608 & 0.2 \\ 2010 / 12 / 05 & 10: 28: 52.641 & 33.182 & -115.602 & 2.5 \\ 2010 / 12 / 05 & 16: 24: 09.641 & 33.183 & -115.599 & 0.1 \\ 2010 / 12 / 05 & 18: 34: 37.641 & 33.157 & -115.648 & 0.1 \\ 2010 / 12 / 05 & 19: 12: 51.641 & 33.118 & -115.610 & 9.1 \\ 2010 / 12 / 05 & 23: 01: 08.641 & 33.188 & -115.599 & 0.8 \\ 2010 / 12 / 05 & 23: 02: 03.641 & 33.176 & -115.608 & 0.4 \\ 2010 / 12 / 05 & 23: 02: 29.641 & 33.188 & -115.599 & 0.8 \\ 2010 / 12 / 05 & 23: 03: 20.641 & 33.188 & -115.599 & 0.8 \\ 2010 / 12 / 05 & 23: 06: 44.641 & 33.188 & -115.599 & 0.8 \\ 2010 / 12 / 05 & 23: 09: 05.641 & 33.188 & -115.599 & 0.8 \\ 2010 / 12 / 05 & 23: 15: 36.641 & 33.188 & -115.599 & 0.8 \\ 2010 / 12 / 06 & 02: 09: 13.641 & 33.143 & -115.638 & 4.8 \\ 2010 / 12 / 06 & 02: 14: 09.641 & 33.143 & -115.638 & 4.8 \\ 2010 / 12 / 06 & 02: 51: 56.641 & 33.159 & -115.635 & 7.3 \\ 2010 / 12 / 06 & 04: 12: 50.641 & 33.143 & -115.638 & 4.8 \\ 2010 / 12 / 06 & 05: 02: 17.641 & 33.143 & -115.638 & 4.8 \\ 2010 / 12 / 06 & 07: 00: 01.641 & 33.205 & -115.577 & 0.0 \\ 2010 / 12 / 06 & 07: 24: 25.641 & 33.192 & -115.626 & 7.7 \\ 2010 / 12 / 06 & 07: 24: 47.641 & 33.192 & -115.626 & 7.7 \\ 2010 / 12 / 06 & 07: 26: 14.641 & 33.192 & -115.626 & 7.7 \\ 2010 / 12 / 06 & 07: 39: 53.641 & 33.207 & -115.580 & 1.9 \\ 2010 / 12 / 06 & 11: 16: 26.641 & 33.165 & -115.623 & 7.0 \\ 2010 / 12 / 06 & 11: 45: 24.641 & 33.199 & -115.571 & 3.3 \\ 2010 / 12 / 06 & 15: 26: 01.641 & 33.228 & -115.626 & 3.7 \\ 2010 / 12 / 06 & 17: 06: 26.641 & 33.214 & -115.620 & 5.3 \\ 2010 / 12 / 06 & 18: 35: 11.641 & 33.194 & -115.583 & 3.0 \\ 2010 / 12 / 06 & 23: 13: 18.641 & 33.149 & -115.640 & 3.5 \\ 2010 / 12 / 07 & 01: 05: 19.641 & 33.177 & -115.607 & 4.2 \\ 2010 / 12 / 07 & 01: 05: 19.641 & 33.193 & -115.615 & 3.9 \\ 2010 / 12 / 07 & 03: 02: 09.641 & 33.226 & -115.588 & 4.6 \\ 2010 / 12 / 07 & 03: 35: 07.641 & 33.214 & -115.620 & 5.3 \\ 2010 / 12 / 07 & 09: 03: 11.641 & 33.177 & -115.621 & 2.7 \\ 2010 / 12 / 07 & 18: 40: 32.641 & 33.159 & -115.635 & 7.3 \\ 2010 / 12 / 08 & 08: 56: 41.641 & 33.149 & -115.640 & 3.5 \\ 2010 / 12 / 08 & 23: 18: 38.641 & 33.214 & -115.620 & 5.3 \\ 2010 / 12 / 09 & 08: 55: 58.641 & 33.184 & -115.597 & 0.1 \\ 2010 / 12 / 09 & 09: 05: 53.641 & 33.184 & -115.597 & 0.1 \\ 2010 / 12 / 09 & 09: 14: 57.641 & 33.177 & -115.606 & 0.1 \\ 2010 / 12 / 09 & 10: 52: 12.641 & 33.184 & -115.597 & 0.1 \\ 2010 / 12 / 10 & 03: 23: 13.641 & 33.175 & -115.601 & 0.1 \\ 2010 / 12 / 10 & 03: 28: 28.641 & 33.108 & -115.602 & 8.1 \\ 2010 / 12 / 10 & 04: 07: 41.641 & 33.207 & -115.577 & 0.0 \\ 23: 10 & 08: 30: 03.641 & 33.186 & -115.580 & 1.9 \\ 23: 02: 23.641 & 33.190 & -115.612 & 1.0\end{array}$




$\begin{array}{lllll}2010 / 12 / 11 & 01: 04: 32.641 & 33.229 & -115.576 & 3.4 \\ 2010 / 12 / 11 & 02: 03: 05.641 & 33.182 & -115.602 & 2.5 \\ 2010 / 12 / 11 & 06: 10: 06.641 & 33.158 & -115.629 & 6.0 \\ 2010 / 12 / 11 & 06: 13: 21.641 & 33.158 & -115.629 & 6.0 \\ 2010 / 12 / 11 & 07: 01: 15.641 & 33.183 & -115.607 & 0.0 \\ 2010 / 12 / 11 & 07: 01: 29.641 & 33.183 & -115.606 & 0.1 \\ 2010 / 12 / 11 & 10: 11: 37.641 & 33.194 & -115.583 & 3.0 \\ 2010 / 12 / 11 & 10: 12: 21.641 & 33.194 & -115.583 & 3.0 \\ 2010 / 12 / 11 & 10: 12: 58.641 & 33.194 & -115.583 & 3.0 \\ 2010 / 12 / 11 & 10: 13: 40.641 & 33.194 & -115.583 & 3.0 \\ 2010 / 12 / 11 & 10: 20: 07.641 & 33.192 & -115.626 & 7.7 \\ 2010 / 12 / 11 & 11: 52: 09.641 & 33.184 & -115.604 & 0.1 \\ 2010 / 12 / 11 & 16: 07: 26.641 & 33.207 & -115.580 & 1.9 \\ 2010 / 12 / 11 & 16: 08: 01.641 & 33.207 & -115.580 & 1.9 \\ 2010 / 12 / 11 & 16: 10: 58.641 & 33.207 & -115.580 & 1.9 \\ 2010 / 12 / 11 & 16: 15: 18.641 & 33.207 & -115.580 & 1.9 \\ 2010 / 12 / 11 & 16: 16: 09.641 & 33.207 & -115.580 & 1.9 \\ 2010 / 12 / 11 & 18: 36: 54.641 & 33.208 & -115.582 & 2.5 \\ 2010 / 12 / 11 & 22: 31: 00.641 & 33.149 & -115.640 & 3.5 \\ 2010 / 12 / 12 & 10: 05: 51.641 & 33.176 & -115.604 & 0.5 \\ 2010 / 12 / 13 & 15: 31: 58.641 & 33.183 & -115.599 & 0.1 \\ 2010 / 12 / 13 & 15: 33: 40.641 & 33.203 & -115.578 & 2.2 \\ 2010 / 12 / 13 & 16: 35: 48.641 & 33.193 & -115.615 & 3.9 \\ 2010 / 12 / 13 & 19: 02: 41.641 & 33.193 & -115.615 & 3.9 \\ 2010 / 12 / 13 & 19: 13: 59.641 & 33.117 & -115.613 & 7.9 \\ 2010 / 12 / 13 & 19: 29: 36.641 & 33.117 & -115.613 & 7.9 \\ 2010 / 12 / 13 & 19: 30: 08.641 & 33.117 & -115.613 & 7.9 \\ 2010 / 12 / 13 & 19: 33: 02.641 & 33.117 & -115.613 & 7.9 \\ 2010 / 12 / 13 & 20: 18: 28.641 & 33.117 & -115.613 & 7.9 \\ 2010 / 12 / 14 & 11: 35: 27.641 & 33.141 & -115.641 & 4.5 \\ 2010 / 12 / 14 & 12: 52: 44.641 & 33.231 & -115.605 & 4.8 \\ 2010 / 12 / 14 & 15: 24: 50.641 & 33.226 & -115.588 & 4.6 \\ 2010 / 12 / 14 & 16: 20: 17.641 & 33.181 & -115.610 & 0.9 \\ 2010 / 12 / 14 & 16: 41: 24.641 & 33.118 & -115.610 & 9.1 \\ 2010 / 12 / 14 & 20: 37: 12.641 & 33.182 & -115.602 & 2.5 \\ 2010 / 12 / 14 & 22: 38: 57.641 & 33.159 & -115.641 & 0.9 \\ 2010 / 12 / 14 & 22: 40: 13.641 & 33.157 & -115.668 & 0.1 \\ 2010 / 12 / 14 & 23: 48: 14.641 & 33.179 & -115.594 & 1.5 \\ 2010 / 12 / 15 & 01: 18: 03.641 & 33.183 & -115.599 & 0.1 \\ 2010 / 12 / 15 & 01: 18: 39.641 & 33.193 & -115.583 & 5.9 \\ 2010 / 12 / 15 & 01: 18: 58.641 & 33.188 & -115.573 & 0.2 \\ 2010 / 12 / 15 & 01: 19: 24.641 & 33.234 & -115.604 & 4.8 \\ 2010 / 12 / 15 & 01: 25: 05.641 & 33.188 & -115.573 & 0.2 \\ 20 / 12 / 15 & 01: 28: 24.641 & 33.177 & -115.599 & 0.1 \\ 04: 43: 43.641 & 33.183 & -115.599 & 0.1\end{array}$




$\begin{array}{lllll}2010 / 12 / 15 & 04: 43: 55.641 & 33.183 & -115.599 & 0.1 \\ 2010 / 12 / 15 & 04: 44: 19.641 & 33.188 & -115.573 & 0.2 \\ 2010 / 12 / 15 & 04: 45: 17.641 & 33.183 & -115.599 & 0.1 \\ 2010 / 12 / 15 & 04: 46: 29.641 & 33.183 & -115.599 & 0.1 \\ 2010 / 12 / 15 & 04: 46: 49.641 & 33.184 & -115.605 & 0.1 \\ 2010 / 12 / 15 & 04: 48: 08.641 & 33.183 & -115.599 & 0.1 \\ 2010 / 12 / 15 & 04: 54: 10.641 & 33.177 & -115.606 & 0.1 \\ 2010 / 12 / 15 & 08: 27: 42.641 & 33.177 & -115.621 & 2.7 \\ 2010 / 12 / 15 & 09: 37: 30.641 & 33.176 & -115.608 & 0.4 \\ 2010 / 12 / 15 & 10: 03: 06.641 & 33.192 & -115.626 & 7.7 \\ 2010 / 12 / 15 & 11: 46: 05.641 & 33.188 & -115.573 & 0.2 \\ 2010 / 12 / 15 & 11: 46: 19.641 & 33.193 & -115.583 & 5.9 \\ 2010 / 12 / 15 & 20: 27: 48.641 & 33.118 & -115.610 & 9.1 \\ 2010 / 12 / 15 & 20: 34: 59.641 & 33.108 & -115.602 & 8.1 \\ 2010 / 12 / 15 & 23: 46: 12.641 & 33.108 & -115.602 & 8.1 \\ 2010 / 12 / 16 & 09: 46: 52.641 & 33.145 & -115.634 & 4.8 \\ 2010 / 12 / 16 & 09: 47: 57.641 & 33.160 & -115.633 & 4.6 \\ 2010 / 12 / 16 & 16: 14: 47.641 & 33.139 & -115.602 & 7.0 \\ 2010 / 12 / 16 & 16: 28: 24.641 & 33.139 & -115.602 & 7.0 \\ 2010 / 12 / 16 & 18: 39: 41.641 & 33.176 & -115.608 & 0.4 \\ 2010 / 12 / 16 & 21: 47: 48.641 & 33.170 & -115.600 & 1.1 \\ 2010 / 12 / 16 & 23: 13: 24.641 & 33.174 & -115.604 & 0.1 \\ 2010 / 12 / 17 & 02: 54: 56.641 & 33.183 & -115.617 & 4.3 \\ 2010 / 12 / 17 & 06: 40: 37.641 & 33.175 & -115.601 & 0.1 \\ 2010 / 12 / 17 & 10: 06: 45.641 & 33.231 & -115.605 & 4.8 \\ 2010 / 12 / 17 & 10: 11: 38.641 & 33.156 & -115.652 & 0.2 \\ 2010 / 12 / 18 & 10: 01: 05.641 & 33.201 & -115.544 & 3.4 \\ 2010 / 12 / 19 & 00: 43: 24.641 & 33.193 & -115.615 & 3.9 \\ 2010 / 12 / 19 & 06: 29: 58.641 & 33.208 & -115.631 & 5.0 \\ 2010 / 12 / 19 & 11: 53: 19.641 & 33.181 & -115.659 & 6.0 \\ 2010 / 12 / 19 & 14: 30: 56.641 & 33.197 & -115.644 & 3.0 \\ 2010 / 12 / 20 & 03: 25: 13.641 & 33.186 & -115.624 & 2.5 \\ 2010 / 12 / 20 & 03: 47: 05.641 & 33.186 & -115.624 & 2.5 \\ 2010 / 12 / 20 & 03: 48: 24.641 & 33.186 & -115.624 & 2.5 \\ 2010 / 12 / 20 & 03: 49: 03.641 & 33.186 & -115.624 & 2.5 \\ 2010 / 12 / 20 & 03: 49: 53.641 & 33.220 & -115.571 & 5.3 \\ 2010 / 12 / 20 & 04: 59: 31.641 & 33.182 & -115.602 & 2.5 \\ 2010 / 12 / 21 & 06: 15: 31.641 & 33.196 & -115.587 & 5.3 \\ 2010 / 12 / 21 & 09: 03: 32.641 & 33.176 & -115.609 & 2.1 \\ 2010 / 12 / 21 & 14: 18: 29.641 & 33.161 & -115.644 & 1.0 \\ 2010 / 12 / 21 & 17: 01: 52.641 & 33.208 & -115.631 & 5.0 \\ 2010 / 12 / 21 & 22: 26: 40.641 & 33.176 & -115.659 & 6.0 \\ 22: 27: 15.64 & 22: 27: 41.641 & 33.176 & -115.609 & 2.1\end{array}$




\begin{tabular}{|c|c|c|c|}
\hline 2010/12/21 & $22: 42: 31.641$ & 33.176 & -115.604 \\
\hline $2010 / 12 / 21$ & $22: 47: 53.641$ & 33.176 & -115.604 \\
\hline $2010 / 12 / 21$ & $22: 53: 03.641$ & 33.176 & -115.609 \\
\hline $2010 / 12 / 21$ & $23: 00: 31.641$ & 33.176 & -115.604 \\
\hline $2010 / 12 / 22$ & $03: 03: 01.641$ & 33.139 & -115.602 \\
\hline $2010 / 12 / 22$ & 03:03:02.641 & 33.108 & -115.602 \\
\hline $2010 / 12 / 22$ & $05: 46: 18.641$ & 33.193 & -115.583 \\
\hline $2010 / 12 / 23$ & $17: 54: 21.641$ & 33.172 & -115.610 \\
\hline $2010 / 12 / 23$ & $17: 54: 39.641$ & 33.176 & -115.609 \\
\hline $2010 / 12 / 23$ & $21: 39: 57.641$ & 33.170 & -115.626 \\
\hline $2010 / 12 / 24$ & $06: 12: 50.641$ & 33.196 & -115.559 \\
\hline $2010 / 12 / 24$ & 08:39:17.641 & 33.182 & -115.603 \\
\hline $2010 / 12 / 24$ & $16: 09: 54.641$ & 33.202 & -115.575 \\
\hline $2010 / 12 / 25$ & $00: 24: 20.641$ & 33.175 & -115.601 \\
\hline $2010 / 12 / 25$ & $00: 35: 49.641$ & 33.170 & -115.600 \\
\hline $2010 / 12 / 25$ & 01:06:13.641 & 33.175 & -115.601 \\
\hline $2010 / 12 / 25$ & 04:04:30.641 & 33.183 & -115.617 \\
\hline $2010 / 12 / 25$ & $04: 54: 54.641$ & 33.139 & -115.602 \\
\hline $2010 / 12 / 25$ & $07: 58: 12.641$ & 33.198 & -115.571 \\
\hline $2010 / 12 / 25$ & $09: 45: 58.641$ & 33.179 & -115.614 \\
\hline $2010 / 12 / 25$ & $10: 06: 38.641$ & 33.187 & -115.608 \\
\hline $2010 / 12 / 25$ & 10:08:09.641 & 33.176 & -115.604 \\
\hline $2010 / 12 / 25$ & $10: 54: 12.641$ & 33.248 & -115.633 \\
\hline $2010 / 12 / 25$ & $14: 07: 32.641$ & 33.157 & -115.668 \\
\hline $2010 / 12 / 25$ & $20: 35: 14.641$ & 33.140 & -115.636 \\
\hline $2010 / 12 / 25$ & $20: 37: 45.641$ & 33.140 & -115.636 \\
\hline $2010 / 12 / 26$ & 03:07:20.641 & 33.182 & -115.602 \\
\hline $2010 / 12 / 26$ & $06: 54: 33.641$ & 33.117 & -115.613 \\
\hline $2010 / 12 / 26$ & $06: 55: 48.641$ & 33.117 & -115.613 \\
\hline $2010 / 12 / 26$ & $06: 58: 59.641$ & 33.108 & -115.602 \\
\hline $2010 / 12 / 26$ & $08: 57: 49.641$ & 33.175 & -115.601 \\
\hline $2010 / 12 / 26$ & 08:58:10.641 & 33.175 & -115.601 \\
\hline $2010 / 12 / 26$ & $11: 39: 56.641$ & 33.192 & -115.579 \\
\hline $2010 / 12 / 26$ & $11: 56: 29.641$ & 33.130 & -115.634 \\
\hline $2010 / 12 / 26$ & $12: 05: 00.641$ & 33.199 & -115.571 \\
\hline $2010 / 12 / 26$ & $15: 10: 18.641$ & 33.188 & -115.573 \\
\hline $2010 / 12 / 26$ & $15: 10: 41.641$ & 33.188 & -115.573 \\
\hline $2010 / 12 / 26$ & $15: 11: 03.641$ & 33.188 & -115.573 \\
\hline $2010 / 12 / 26$ & $15: 11: 18.641$ & 33.188 & -115.573 \\
\hline $2010 / 12 / 26$ & $15: 12: 51.641$ & 33.188 & -115.573 \\
\hline $2010 / 12 / 26$ & $22: 15: 16.641$ & 33.177 & -115.606 \\
\hline $2010 / 12 / 26$ & $23: 44: 19.641$ & 33.193 & -115.615 \\
\hline $2010 / 12 / 26$ & $23: 47: 29.641$ & 33.193 & -115.615 \\
\hline $2010 / 12 / 26$ & $23: 57: 03.641$ & 33.193 & -115.615 \\
\hline $2010 / 12 / 27$ & 02:00:12.641 & 33.175 & -115.603 \\
\hline $2010 / 12 / 27$ & $03: 58: 41.641$ & 33.108 & -115.602 \\
\hline
\end{tabular}




$\begin{array}{lllll}2010 / 12 / 27 & 05: 22: 46.641 & 33.183 & -115.677 & 0.5 \\ 2010 / 12 / 27 & 06: 05: 39.641 & 33.193 & -115.615 & 3.9 \\ 2010 / 12 / 27 & 07: 30: 34.641 & 33.100 & -115.590 & 14.6 \\ 2010 / 12 / 27 & 11: 01: 49.641 & 33.174 & -115.603 & 0.1 \\ 2010 / 12 / 27 & 17: 55: 59.641 & 33.115 & -115.585 & 6.5 \\ 2010 / 12 / 27 & 21: 44: 54.641 & 33.184 & -115.604 & 0.1 \\ 2010 / 12 / 27 & 22: 51: 19.641 & 33.193 & -115.615 & 3.9 \\ 2010 / 12 / 27 & 23: 41: 16.641 & 33.193 & -115.615 & 3.9 \\ 2010 / 12 / 27 & 23: 42: 33.641 & 33.193 & -115.615 & 3.9 \\ 2010 / 12 / 27 & 23: 43: 54.641 & 33.193 & -115.615 & 3.9 \\ 2010 / 12 / 27 & 23: 44: 23.641 & 33.193 & -115.615 & 3.9 \\ 2010 / 12 / 28 & 00: 02: 46.641 & 33.209 & -115.590 & 3.1 \\ 2010 / 12 / 28 & 00: 07: 09.641 & 33.193 & -115.615 & 3.9 \\ 2010 / 12 / 28 & 00: 11: 47.641 & 33.203 & -115.578 & 2.2 \\ 2010 / 12 / 28 & 00: 16: 27.641 & 33.209 & -115.590 & 3.1 \\ 2010 / 12 / 28 & 00: 19: 40.641 & 33.176 & -115.608 & 0.4 \\ 2010 / 12 / 28 & 00: 21: 37.641 & 33.176 & -115.608 & 0.4 \\ 2010 / 12 / 28 & 00: 43: 09.641 & 33.193 & -115.615 & 3.9 \\ 2010 / 12 / 28 & 01: 18: 40.641 & 33.193 & -115.615 & 3.9 \\ 2010 / 12 / 28 & 01: 19: 23.641 & 33.193 & -115.615 & 3.9 \\ 2010 / 12 / 28 & 01: 20: 51.641 & 33.193 & -115.615 & 3.9 \\ 2010 / 12 / 28 & 01: 21: 06.641 & 33.193 & -115.615 & 3.9 \\ 2010 / 12 / 28 & 01: 21: 57.641 & 33.193 & -115.615 & 3.9 \\ 2010 / 12 / 28 & 01: 24: 05.641 & 33.193 & -115.615 & 3.9 \\ 2010 / 12 / 28 & 01: 25: 38.641 & 33.193 & -115.615 & 3.9 \\ 2010 / 12 / 28 & 01: 30: 57.641 & 33.193 & -115.615 & 3.9 \\ 2010 / 12 / 28 & 01: 31: 26.641 & 33.193 & -115.615 & 3.9 \\ 2010 / 12 / 28 & 01: 42: 34.641 & 33.193 & -115.615 & 3.9 \\ 2010 / 12 / 28 & 01: 43: 14.641 & 33.208 & -115.582 & 2.5 \\ 2010 / 12 / 28 & 01: 44: 16.641 & 33.193 & -115.615 & 3.9 \\ 2010 / 12 / 28 & 01: 53: 03.641 & 33.193 & -115.615 & 3.9 \\ 2010 / 12 / 28 & 01: 56: 34.641 & 33.193 & -115.615 & 3.9 \\ 2010 / 12 / 28 & 01: 57: 38.641 & 33.193 & -115.615 & 3.9 \\ 2010 / 12 / 28 & 01: 58: 08.641 & 33.193 & -115.615 & 3.9 \\ 2010 / 12 / 28 & 01: 59: 07.641 & 33.193 & -115.615 & 3.9 \\ 2010 / 12 / 28 & 01: 59: 58.641 & 33.176 & -115.606 & 6.3 \\ 2010 / 12 / 28 & 02: 00: 41.641 & 33.193 & -115.615 & 3.9 \\ 2010 / 12 / 28 & 02: 01: 25.641 & 33.193 & -115.615 & 3.9 \\ 2010 / 12 / 28 & 02: 02: 18.641 & 33.193 & -115.615 & 3.9 \\ 2010 / 12 / 28 & 02: 05: 39.641 & 33.193 & -115.615 & 3.9 \\ 2010 / 12 / 28 & 02: 17: 04.641 & 33.193 & -115.615 & 3.9 \\ 2010 / 12 / 28 & 02: 21: 54.641 & 33.193 & -115.615 & 3.9 \\ 2010 / 12 / 28 & 02: 25: 30.641 & 33.193 & -115.615 & 3.9 \\ 2010 / 12 / 28 & 02: 25: 59.641 & 33.193 & -115.615 & 3.9 \\ 2010 / 12 / 28 & 02: 26: 49.641 & 33.193 & -115.615 & 3.9 \\ 2010 / 12 / 28 & 02: 27: 27.641 & 33.193 & -115.615 & 3.9\end{array}$




$\begin{array}{lllll}2010 / 12 / 28 & 02: 31: 00.641 & 33.202 & -115.575 & 3.8 \\ 2010 / 12 / 28 & 02: 35: 23.641 & 33.193 & -115.615 & 3.9 \\ 2010 / 12 / 28 & 02: 38: 36.641 & 33.193 & -115.615 & 3.9 \\ 2010 / 12 / 28 & 02: 40: 20.641 & 33.193 & -115.615 & 3.9 \\ 2010 / 12 / 28 & 02: 40: 39.641 & 33.183 & -115.617 & 4.3 \\ 2010 / 12 / 28 & 02: 41: 08.641 & 33.193 & -115.615 & 3.9 \\ 2010 / 12 / 28 & 02: 41: 32.641 & 33.193 & -115.615 & 3.9 \\ 2010 / 12 / 28 & 02: 41: 55.641 & 33.193 & -115.615 & 3.9 \\ 2010 / 12 / 28 & 02: 42: 21.641 & 33.183 & -115.617 & 4.3 \\ 2010 / 12 / 28 & 02: 42: 47.641 & 33.193 & -115.615 & 3.9 \\ 2010 / 12 / 28 & 02: 43: 14.641 & 33.183 & -115.617 & 4.3 \\ 2010 / 12 / 28 & 02: 43: 27.641 & 33.183 & -115.617 & 4.3 \\ 2010 / 12 / 28 & 02: 44: 22.641 & 33.193 & -115.615 & 3.9 \\ 2010 / 12 / 28 & 02: 44: 34.641 & 33.193 & -115.615 & 3.9 \\ 2010 / 12 / 28 & 02: 44: 49.641 & 33.218 & -115.639 & 3.0 \\ 2010 / 12 / 28 & 02: 45: 30.641 & 33.186 & -115.593 & 0.1 \\ 2010 / 12 / 28 & 02: 46: 02.641 & 33.184 & -115.608 & 0.3 \\ 2010 / 12 / 28 & 02: 46: 35.641 & 33.175 & -115.610 & 2.4 \\ 2010 / 12 / 28 & 02: 46: 59.641 & 33.184 & -115.608 & 0.3 \\ 2010 / 12 / 28 & 02: 48: 14.641 & 33.196 & -115.587 & 4.5 \\ 2010 / 12 / 28 & 02: 49: 09.641 & 33.193 & -115.615 & 3.9 \\ 2010 / 12 / 28 & 02: 49: 39.641 & 33.193 & -115.615 & 3.9 \\ 2010 / 12 / 28 & 02: 49: 51.641 & 33.193 & -115.615 & 3.9 \\ 2010 / 12 / 28 & 02: 50: 08.641 & 33.193 & -115.615 & 3.9 \\ 2010 / 12 / 28 & 02: 50: 31.641 & 33.193 & -115.615 & 3.9 \\ 2010 / 12 / 28 & 02: 51: 31.641 & 33.183 & -115.617 & 4.3 \\ 2010 / 12 / 28 & 02: 52: 26.641 & 33.118 & -115.610 & 9.1 \\ 2010 / 12 / 28 & 02: 53: 22.641 & 33.193 & -115.615 & 3.9 \\ 2010 / 12 / 28 & 02: 54: 01.641 & 33.193 & -115.615 & 3.9 \\ 2010 / 12 / 28 & 02: 54: 53.641 & 33.193 & -115.615 & 3.9 \\ 2010 / 12 / 28 & 02: 55: 14.641 & 33.193 & -115.615 & 3.9 \\ 2010 / 12 / 28 & 02: 55: 35.641 & 33.193 & -115.615 & 3.9 \\ 2010 / 12 / 28 & 02: 56: 16.641 & 33.188 & -115.609 & 2.3 \\ 2010 / 12 / 28 & 02: 56: 45.641 & 33.193 & -115.615 & 3.9 \\ 2010 / 12 / 28 & 02: 58: 26.641 & 33.183 & -115.617 & 4.3 \\ 2010 / 12 / 28 & 02: 59: 01.641 & 33.176 & -115.609 & 2.1 \\ 2010 / 12 / 28 & 02: 59: 16.641 & 33.213 & -115.584 & 1.3 \\ 2010 / 12 / 28 & 02: 59: 57.641 & 33.173 & -115.609 & 0.1 \\ 2010 / 12 / 28 & 03: 01: 16.641 & 33.179 & -115.614 & 1.8 \\ 2010 / 12 / 28 & 03: 01: 50.641 & 33.193 & -115.615 & 3.9 \\ 2010 / 12 / 28 & 03: 02: 41.641 & 33.184 & -115.601 & 3.5 \\ 2010 / 12 / 28 & 03: 03: 09.641 & 33.180 & -115.609 & 0.0 \\ 2010 / 12 / 28 & 03: 05: 07.64 .641 & 33.193 & -115.615 & 3.9 \\ 28 & 03: 06: 29.641 & 33.187 & -115.615 & 3.9 \\ 28 & 03: 07: 13.641 & 33.108 & -115.602 & 8.1\end{array}$




$\begin{array}{lllll}2010 / 12 / 28 & 03: 07: 33.641 & 33.187 & -115.604 & 3.2 \\ 2010 / 12 / 28 & 03: 09: 27.641 & 33.184 & -115.604 & 0.1 \\ 2010 / 12 / 28 & 03: 10: 40.641 & 33.184 & -115.608 & 0.3 \\ 2010 / 12 / 28 & 03: 13: 25.641 & 33.176 & -115.609 & 2.1 \\ 2010 / 12 / 28 & 03: 13: 56.641 & 33.202 & -115.575 & 3.8 \\ 2010 / 12 / 28 & 03: 15: 19.641 & 33.213 & -115.584 & 1.3 \\ 2010 / 12 / 28 & 03: 15: 51.641 & 33.175 & -115.600 & 1.1 \\ 2010 / 12 / 28 & 03: 16: 05.641 & 33.193 & -115.615 & 3.9 \\ 2010 / 12 / 28 & 03: 17: 06.641 & 33.175 & -115.610 & 2.4 \\ 2010 / 12 / 28 & 03: 18: 45.641 & 33.193 & -115.615 & 3.9 \\ 2010 / 12 / 28 & 03: 19: 05.641 & 33.193 & -115.615 & 3.9 \\ 2010 / 12 / 28 & 03: 20: 28.641 & 33.193 & -115.615 & 3.9 \\ 2010 / 12 / 28 & 03: 20: 41.641 & 33.186 & -115.593 & 0.1 \\ 2010 / 12 / 28 & 03: 20: 59.641 & 33.193 & -115.615 & 3.9 \\ 2010 / 12 / 28 & 03: 23: 44.641 & 33.193 & -115.615 & 3.9 \\ 2010 / 12 / 28 & 03: 26: 06.641 & 33.193 & -115.615 & 3.9 \\ 2010 / 12 / 28 & 03: 27: 04.641 & 33.170 & -115.600 & 1.1 \\ 2010 / 12 / 28 & 03: 27: 46.641 & 33.214 & -115.620 & 5.3 \\ 2010 / 12 / 28 & 03: 28: 37.641 & 33.193 & -115.615 & 3.9 \\ 2010 / 12 / 28 & 03: 28: 48.641 & 33.142 & -115.628 & 10.3 \\ 2010 / 12 / 28 & 03: 29: 14.641 & 33.203 & -115.578 & 2.2 \\ 2010 / 12 / 28 & 03: 29: 35.641 & 33.183 & -115.617 & 4.3 \\ 2010 / 12 / 28 & 03: 31: 28.641 & 33.181 & -115.611 & 0.4 \\ 2010 / 12 / 28 & 03: 32: 04.641 & 33.171 & -115.605 & 0.7 \\ 2010 / 12 / 28 & 03: 34: 07.641 & 33.236 & -115.573 & 5.0 \\ 2010 / 12 / 28 & 03: 36: 39.641 & 33.176 & -115.606 & 6.3 \\ 2010 / 12 / 28 & 03: 39: 16.641 & 33.183 & -115.617 & 4.3 \\ 2010 / 12 / 28 & 03: 42: 32.641 & 33.208 & -115.582 & 2.5 \\ 2010 / 12 / 28 & 03: 42: 51.641 & 33.170 & -115.600 & 1.1 \\ 2010 / 12 / 28 & 03: 43: 25.641 & 33.184 & -115.608 & 0.3 \\ 2010 / 12 / 28 & 03: 45: 56.641 & 33.196 & -115.587 & 5.3 \\ 2010 / 12 / 28 & 03: 47: 48.641 & 33.208 & -115.582 & 2.5 \\ 2010 / 12 / 28 & 03: 48: 17.641 & 33.181 & -115.659 & 6.0 \\ 2010 / 12 / 28 & 03: 49: 22.641 & 33.241 & -115.633 & 3.4 \\ 2010 / 12 / 28 & 03: 51: 50.641 & 33.108 & -115.602 & 8.1 \\ 2010 / 12 / 28 & 03: 56: 15.641 & 33.231 & -115.655 & 4.8 \\ 2010 / 12 / 28 & 03: 59: 49.641 & 33.173 & -115.610 & 1.3 \\ 2010 / 12 / 28 & 04: 00: 56.641 & 33.251 & -115.564 & 0.1 \\ 2010 / 12 / 28 & 04: 01: 27.641 & 33.193 & -115.615 & 3.9 \\ 2010 / 12 / 28 & 04: 07: 47.641 & 33.193 & -115.615 & 3.9 \\ 2010 / 12 / 28 & 04: 11: 09.641 & 33.202 & -115.575 & 3.8 \\ 2010 / 12 / 28 & 04: 14: 03.641 & 33.183 & -115.617 & 4.3 \\ 2010 / 12 / 28 & 04: 22: 05.641 & 33.231 & -115.655 & 4.8 \\ 2010 / 12 / 28 & 04: 22: 17.641 & 33.193 & -115.615 & 3.9 \\ 20 / 12 / 28 & 04: 41: 34.641 & 33.213 & -115.584 & 1.3\end{array}$




$\begin{array}{lllll}2010 / 12 / 28 & 04: 46: 04.641 & 33.173 & -115.610 & 1.3 \\ 2010 / 12 / 28 & 04: 56: 01.641 & 33.193 & -115.615 & 3.9 \\ 2010 / 12 / 28 & 04: 58: 35.641 & 33.193 & -115.615 & 3.9 \\ 2010 / 12 / 28 & 04: 58: 52.641 & 33.193 & -115.615 & 3.9 \\ 2010 / 12 / 28 & 05: 02: 28.641 & 33.201 & -115.544 & 3.4 \\ 2010 / 12 / 28 & 05: 04: 07.641 & 33.193 & -115.615 & 3.9 \\ 2010 / 12 / 28 & 05: 17: 43.641 & 33.193 & -115.615 & 3.9 \\ 2010 / 12 / 28 & 05: 18: 04.641 & 33.193 & -115.615 & 3.9 \\ 2010 / 12 / 28 & 05: 45: 55.641 & 33.100 & -115.590 & 14.6 \\ 2010 / 12 / 28 & 05: 51: 37.641 & 33.193 & -115.615 & 3.9 \\ 2010 / 12 / 28 & 05: 52: 33.641 & 33.186 & -115.593 & 0.1 \\ 2010 / 12 / 28 & 06: 12: 41.641 & 33.208 & -115.582 & 2.5 \\ 2010 / 12 / 28 & 06: 13: 35.641 & 33.115 & -115.585 & 6.5 \\ 2010 / 12 / 28 & 06: 44: 39.641 & 33.183 & -115.599 & 0.1 \\ 2010 / 12 / 28 & 06: 44: 51.641 & 33.183 & -115.599 & 0.1 \\ 2010 / 12 / 28 & 06: 45: 40.641 & 33.183 & -115.599 & 0.1 \\ 2010 / 12 / 28 & 06: 52: 53.641 & 33.183 & -115.599 & 0.1 \\ 2010 / 12 / 28 & 07: 50: 32.641 & 33.173 & -115.610 & 1.3 \\ 2010 / 12 / 28 & 07: 50: 54.641 & 33.190 & -115.612 & 1.0 \\ 2010 / 12 / 28 & 07: 51: 31.641 & 33.190 & -115.612 & 1.0 \\ 2010 / 12 / 28 & 07: 52: 53.641 & 33.190 & -115.612 & 1.0 \\ 2010 / 12 / 28 & 08: 18: 43.641 & 33.181 & -115.611 & 0.4 \\ 2010 / 12 / 28 & 08: 25: 57.641 & 33.197 & -115.614 & 5.3 \\ 2010 / 12 / 28 & 09: 36: 47.641 & 33.193 & -115.583 & 5.9 \\ 2010 / 12 / 28 & 10: 43: 13.641 & 33.190 & -115.608 & 2.2 \\ 2010 / 12 / 28 & 10: 45: 48.641 & 33.199 & -115.617 & 0.0 \\ 2010 / 12 / 28 & 10: 58: 09.641 & 33.172 & -115.611 & 3.7 \\ 2010 / 12 / 28 & 11: 10: 03.641 & 33.157 & -115.680 & 0.2 \\ 2010 / 12 / 28 & 11: 13: 10.641 & 33.157 & -115.680 & 0.2 \\ 2010 / 12 / 28 & 12: 13: 12.641 & 33.193 & -115.615 & 3.9 \\ 2010 / 12 / 28 & 12: 23: 20.641 & 33.193 & -115.615 & 3.9 \\ 2010 / 12 / 28 & 12: 51: 15.641 & 33.208 & -115.582 & 2.5 \\ 2010 / 12 / 28 & 13: 19: 19.641 & 33.108 & -115.602 & 8.1 \\ 2010 / 12 / 28 & 18: 06: 00.641 & 33.202 & -115.575 & 3.8 \\ 2010 / 12 / 28 & 18: 09: 34.641 & 33.179 & -115.614 & 1.8 \\ 2010 / 12 / 28 & 18: 28: 11.641 & 33.202 & -115.575 & 3.8 \\ 2010 / 12 / 28 & 20: 58: 12.641 & 33.213 & -115.584 & 1.3 \\ 2010 / 12 / 29 & 02: 20: 12.641 & 33.176 & -115.618 & 3.7 \\ 2010 / 12 / 29 & 02: 20: 25.641 & 33.208 & -115.582 & 2.5 \\ 2010 / 12 / 29 & 02: 28: 05.641 & 33.196 & -115.587 & 4.5 \\ 2010 / 12 / 29 & 02: 41: 44.641 & 33.202 & -115.575 & 3.8 \\ 2010 / 12 / 29 & 04: 50: 36.641 & 33.184 & -115.608 & 0.3 \\ 2010 / 12 / 29 & 06: 58: 59.641 & 33.181 & -115.665 & 4.2 \\ 2010 / 12 / 29 & 08: 27: 24.641 & 33.118 & -115.610 & 9.1 \\ 2010 / 12 / 29 & 08: 29: 38.641 & 33.188 & -115.599 & 0.8 \\ 2010 / 12 / 29 & 08: 58: 08.641 & 33.108 & -115.602 & 8.1\end{array}$




$\begin{array}{lllll}2010 / 12 / 29 & 09: 32: 08.641 & 33.135 & -115.644 & 6.9 \\ 2010 / 12 / 29 & 10: 08: 36.641 & 33.193 & -115.615 & 3.9 \\ 2010 / 12 / 29 & 12: 43: 13.641 & 33.208 & -115.582 & 2.5 \\ 2010 / 12 / 29 & 13: 02: 25.641 & 33.193 & -115.615 & 3.9 \\ 2010 / 12 / 29 & 14: 21: 57.641 & 33.187 & -115.608 & 0.2 \\ 2010 / 12 / 29 & 14: 53: 57.641 & 33.135 & -115.644 & 6.9 \\ 2010 / 12 / 29 & 17: 14: 25.641 & 33.175 & -115.601 & 0.1 \\ 2010 / 12 / 29 & 22: 23: 05.641 & 33.117 & -115.613 & 7.9 \\ 2010 / 12 / 30 & 01: 12: 01.641 & 33.189 & -115.576 & 0.1 \\ 2010 / 12 / 30 & 01: 13: 10.641 & 33.202 & -115.575 & 3.8 \\ 2010 / 12 / 30 & 04: 51: 18.641 & 33.158 & -115.638 & 0.4 \\ 2010 / 12 / 30 & 08: 33: 37.641 & 33.108 & -115.602 & 8.1 \\ 2010 / 12 / 30 & 11: 38: 41.641 & 33.212 & -115.590 & 7.2 \\ 2010 / 12 / 30 & 11: 55: 51.641 & 33.202 & -115.575 & 3.8 \\ 2010 / 12 / 30 & 15: 16: 19.641 & 33.190 & -115.574 & 3.1 \\ 2010 / 12 / 30 & 15: 17: 57.641 & 33.185 & -115.569 & 3.7 \\ 2010 / 12 / 30 & 16: 13: 44.641 & 33.157 & -115.680 & 0.2 \\ 2010 / 12 / 30 & 16: 14: 11.641 & 33.157 & -115.680 & 0.2 \\ 2010 / 12 / 30 & 19: 41: 54.641 & 33.172 & -115.611 & 3.7 \\ 2010 / 12 / 30 & 19: 46: 44.641 & 33.218 & -115.639 & 3.0 \\ 2010 / 12 / 30 & 20: 22: 37.641 & 33.172 & -115.611 & 3.7 \\ 2010 / 12 / 30 & 21: 21: 26.641 & 33.149 & -115.640 & 3.5 \\ 2010 / 12 / 31 & 06: 24: 01.641 & 33.193 & -115.615 & 3.9 \\ 2010 / 12 / 31 & 08: 19: 32.641 & 33.183 & -115.606 & 0.1 \\ 2010 / 12 / 31 & 10: 47: 32.641 & 33.172 & -115.611 & 3.7 \\ 2010 / 12 / 31 & 13: 20: 29.641 & 33.170 & -115.654 & 0.0 \\ 2010 / 12 / 31 & 13: 21: 11.641 & 33.170 & -115.654 & 0.0 \\ 2010 / 12 / 31 & 19: 33: 19.641 & 33.172 & -115.611 & 3.7 \\ 2010 / 12 / 31 & 22: 30: 31.641 & 33.177 & -115.606 & 0.1\end{array}$

